\title{
Beeinflussung des laminar-turbulenten Grenzschichtumschlags durch kontrollierte Anregung stationärer Querströmungsinstabilitäten
}

\section{Dissertation}

zur Erlangung des mathematisch-naturwissenschaftlichen Doktorgrades „Doctor rerum naturalium“ der Georg-August-Universität Göttingen im Promotionsprogramm ProPhys
der Georg-August University School of Science (GAUSS)

vorgelegt von

Hans Peter Barth

aus Göttingen

Göttingen, 2021 


\section{Betreuungsausschuss}

\section{Prof. Dr. Andreas Dillmann,}

Georg-August-Universität Göttingen, Institut für Dynamik komplexer Systeme und Deutsches Zentrum für Luft- und Raumfahrt e.V., Institut für Aerodynamik und Strömungstechnik

\section{Prof. Dr. Martin Rein,}

Georg-August-Universität Göttingen, Institut für Dynamik komplexer Systeme und Deutsches Zentrum für Luft- und Raumfahrt e.V., Institut für Aerodynamik und Strömungstechnik

\section{Mitglieder der Prüfungskommission}

Referent: Prof. Dr. Andreas Dillmann, Georg-August-Universität Göttingen, Institut für Dynamik komplexer Systeme und Deutsches Zentrum für Luft- und Raumfahrt e.V., Institut für Aerodynamik und Strömungstechnik

Korreferent: Prof. Dr. Martin Rein,

Georg-August-Universität Göttingen, Institut für Dynamik komplexer Systeme und Deutsches Zentrum für Luft- und Raumfahrt e.V., Institut für Aerodynamik und Strömungstechnik

Weitere Mitglieder der Prüfungskommission:

Prof. Dr. Wolfgang Glatzel, Georg-August-Universität Göttingen, Institut für Astrophysik

\section{Prof. Dr. Stephan Herminghaus,}

Georg-August-Universität Göttingen, Institut für Dynamik komplexer Systeme und Max-Planck-Institut für Dynamik und Selbstorganisation

\section{Prof. Dr. Wolfram Kollatschny,}

Georg-August-Universität Göttingen, Institut für Astrophysik

\section{Prof. Dr. Andreas Tilgner,}

Georg-August-Universität Göttingen, Institut für Geophysik 


\section{Kurzbeschreibung}

Die Verzögerung des laminar-turbulenten Grenzschichtumschlags (Transition), bspw. an Flugzeugtragflügeln, ist insbesondere wegen der möglichen Reduktion des Reibungswiderstands von Interesse. Zur Untersuchung einer nichtlinearen Verzögerungsmethode für querströmungsdominierte Transition namens Upstream Flow Deformation (UFD) wurde eine neue experimentelle Konfiguration mit einer instabilen dreidimensionalen Grenzschicht ausgelegt und aufgebaut. Als Vorbild diente die experimentelle Konfiguration, die in der Literatur als DLR-Prinzipexperiment der schiebenden ebenen Platte bekannt ist. Zur UFD-Methode ist bekannt, dass zusätzliche, künstlich angeregte stationäre Querströmungsinstabilitäten (QSI) zu einer Transitionsverzögerung führen können. Im Rahmen dieser Arbeit wurden zwei Konzepte für eine solche künstliche Anregung untersucht. Die stationären QSI wurden dabei in einem Fall durch spannweitig periodische lokale Heizung der Modelloberfläche angeregt. Im zweiten Fall wurde dafür eine spannweitige Reihe von in der Oberfläche integrierten, rotierenden Scheiben verwendet. Beide Konzepte wurden bisher in diesem Kontext nicht untersucht und die Frage ihrer Anwendbarkeit in der UFD-Methode war ein zentraler Teil der Zielsetzung. Es wurden rasterförmige Grenzschichtmessungen mithilfe der Hitzdrahtanemometrie im Ein-Meter-Windkanal in Göttingen durchgeführt, deren Interpretation durch lineare lokale Stabilitätsuntersuchungen (LST) unterstützt wurde. Für beide Konzepte war die künstliche Anregung stationärer QSI erfolgreich und wurde jeweils in Abhängigkeit eines geeigneten Steuerparameters quantifiziert. Bei der Heizanregung wurde dabei auch der Einfluss der Aktuierungsposition und der Länge der Heizelemente untersucht. Bei der Rotationsanregung wurde der Einfluss des Drehsinns der Scheiben untersucht. Bei der Heizanregung wurde in manchen Fällen eine Reduktion der Amplitude der eigentlich anzuregenden stationären QSI beobachtet. Dies wurde als destruktive Superposition der Heizanregung mit der Anregung durch die Rauigkeitseigenschaft der Heizelemente interpretiert. Eine Transitionsverzögerung wurde nicht erreicht. Durch detaillierte Messungen konnten die Gründe dafür ausreichend nachvollzogen werden. Die prinzipielle Anwendbarkeit beider Anregungskonzepte in der UFD-Methode wurde bestätigt. 


\begin{abstract}
The delay of laminar-turbulent transition, e.g. on aircraft wings, is of special interest because of the possible drag reduction. To study a nonlinear method for delaying crossflow-dominated transition called Upstream Flow Deformation (UFD), a new experimental configuration with an unstable, three-dimensional boundary-layer was designed and set up. It was based on the experimental configuration known in literature as DLR swept-wing reference experiment. In the UFD context, it is known that additional artificially excited stationary crossflow instabilities (CFI) can lead to delayed transition. In the scope of this thesis, two concepts for such an artificial excitation were studied. In one case, stationary CFI were excited by spanwise periodic heating of the model surface. In the other case, a spanwise row of rotating disks, integrated into the model surface, was used. Both concepts have not been studied within this context and the question of their applicability within the UFD method was a central part of the thesis goal. Grid-like boundary-layer measurements were conducted using hot-wire anemometry in the One-Meter wind tunnel in Göttingen and their interpretation was supported by linear local stability analysis (LST). For both concepts the artificial excitation of stationary CFI was successful and was quantified relative to a respective suitable control parameter. In addition to this, the influence of actuation location and the length of the heating elements was studied for heating actuation. For rotational actuation, the influence of the rotational direction was studied. With heating actuation, a reduction of the CFI amplitude, which was to be excited, was observed in some cases. This was interpreted as a destructive superposition of the excitation by heating and the excitation by roughness. Transition delay was not achieved but detailed measurements led to a sufficient understanding of the reasons for this. In principle, the applicability of both excitation concepts within the UFD method was confirmed.
\end{abstract}




\section{Vorwort}

Die vorliegende Arbeit entstand während meiner Tätigkeit als wissenschaftlicher Mitarbeiter in der Abteilung Hochgeschwindigkeitskonfigurationen des Instituts für Aerodynamik und Strömungstechnik des Deutschen Zentrums für Luft- und Raumfahrt (DLR) in Göttingen.

Herrn Prof. Dr. Andreas Dillmann und Herrn Prof. Dr. Martin Rein möchte ich für die Übernahme des Referates und Korreferates dieser Dissertation danken.

Mein besonderer Dank gilt auch Herrn Dr. Henning Rosemann als Abteilungsleiter für die ursprüngliche Ermöglichung dieser Arbeit und gemeinsam mit Herrn Dr. Stefan Hein für die Anregung des Themas. Herrn Dr. Stefan Hein möchte ich besonders für die ausgezeichnete fachliche Betreuung der Arbeit, interessante Diskussionen und die Einführung in die komplexe Welt der Transition danken. Auch dem späteren Abteilungsleiter, Herrn Dr. Kai Richter, möchte ich herzlich dafür danken, in seiner Abteilung die Arbeit fertigstellen zu können und durch das geeignete Umfeld viel zum Gelingen beigetragen zu haben.

Der gesamten Abteilung Hochgeschwindigkeitskonfigurationen und insbesondere der Transitionsgruppe gilt mein besonderer Dank für die angenehme Arbeitsatmosphäre, viele interessante Diskussionen, Anregungen und Hilfe.

Besonderer Dank gilt auch Herrn Uwe Becker, der die technische Konstruktion der Windkanalmodelle, deren Halterung und vieler Zubehörteile übernommen hat. Herrn Hartmut Haselmeyer danke ich für die tatkräftige Unterstützung bei vielen Problemlösungen bzgl. der Messtechnik. Den Herren Karl Borchert und Lothar Schuster gilt mein Dank für die regelmäßige und oft kurzfristige Unterstützung bei der Modellvorbereitung, technischen Realisierung, Reparatur und für viele lustige Stunden im Labor. Herrn Dr. Erich Schülein danke ich herzlich für die Unterstützung bei der Anwendung der Infrarotkamera und für die Überlassung seiner Software zur perspektivischen Entzerrung der Bilder.

Den Kollegen von SHT gilt mein Dank für die hochwertige Fertigung diverser Modelle und Bauteile sowie für die Nutzung der Koordinatenmessmaschine. 
Den Kollegen der Abteilung Hubschrauber gilt mein Dank für die häufige und langwierige Nutzung ihres Windkanals und Herrn Markus Krebs für die Unterstützung beim Auf- und Abbau, aber auch vielen anderen Aufgaben am Windkanal.

Abschließend möchte ich meiner Familie, Freunden und meiner Freundin Stefanie für ihre Geduld und Unterstützung während der Dauer der Arbeit danken. 


\section{Inhaltsverzeichnis}

1 Einleitung 1

1.1 Einführung . . . . . . . . . . . . . . . . . . 1

1.2 Zielsetzung und Überblick . . . . . . . . . . . . . . . . . 2

2 Hintergrund und Stand der Forschung $\quad 7$

2.1 Die Grundströmung . . . . . . . . . . . . . . . . . . . . . . 8

2.2 Lineare Stabilitätstheorie . . . . . . . . . . . . . . . . 10

2.3 Der querströmungsdominierte inkompressible Transitionsprozess 13

2.4 Verzögerung der querströmungsdominierten Transition . . . . . 23

2.4.1 Upstream Flow Deformation . . . . . . . . . . . . 23

2.5 Gezielte Anregung stationärer Querströmungsinstabilitäten . . 26

2.5.1 Diskrete Einzelrauigkeiten . . . . . . . . . . . 26

2.5 .2 Lokale Heizung . . . . . . . . . . . . . . . . . . . 27

2.5.3 Rotationsaktuierung . . . . . . . . . . . . . . . 29

3 Numerische Methoden $\quad 31$

3.1 Berechnung der Grundströmung . . . . . . . . . . . . . . . . 32

3.2 Lineare Stabilitätsuntersuchungen . . . . . . . . . . . . . 34

4 Die experimentelle Konfiguration $\quad 37$

4.1 Das ursprüngliche Prinzipexperiment der schiebenden ebenen Platte . . . . . . . . . . . . . . 37

4.2 Zielsetzung der Neuauslegung . . . . . . . . . . . . . . . . . 41

4.3 Vorgehensweise der Neuauslegung . . . . . . . . . . . . . . . . 42

4.4 Das neu ausgelegte Prinzipexperiment der schiebenden ebenen Platte .................... . 44 44

4.5 Aktuatoren . . . . . . . . . . . . . . . 47

4.5.1 Heizaktuator . . . . . . . . . . . . . . . 48

4.5 .2 Rotationsaktuator . . . . . . . . . . . . . . 52 
5 Experimentelle Methoden und Versuchstechnik 57

5.1 Hitzdrahtmessungen . . . . . . . . . . . . . . . . 57

5.1 .1 Kalibrierung und Auswertung . . . . . . . . . . . 62

5.1 .2 Hitzdrahtkalibrator . . . . . . . . . . . . . 66

5.1.3 Durchgeführter Ablauf der Kalibrierung . . . . . . . . . . 71

5.1 .4 Messablauf . . . . . . . . . . . . . . . 73

5.1 .5 Datenerfassung . . . . . . . . . . . . . . 75

5.1.6 Fehlerbetrachtung für Hitzdrahtmessungen . . . . . . . 76

5.2 Der Ein-Meter-Windkanal Göttingen . . . . . . . . . . . . . . 82

5.2.1 Charakterisierung der instationären Anströmung . . . . . 84

5.2.2 Reproduzierbarkeit der Strömungsbedingungen . . . . . . 87

5.3 Druckmessungen . . . . . . . . . . . . . . . . . . . . 89

5.4 Infrarot-Thermografie . . . . . . . . . . . . . . . . 90

6 Die transitionelle Strömung im neu ausgelegten $\begin{array}{ll}\text { Prinzipexperiment } & 93\end{array}$

6.1 Grundlegende Messungen . . . . . . . . . . . . . . . . . . . . . 94

6.1.1 Die Gültigkeit der Annahme unendlich schiebender

Bedingungen . . . . . . . . . . . . . . . 94

6.1.2 Strömung am Grenzschichtrand und virtuelle Anströmungsbedingungen . . . . . . . . . . . . 96

6.1.3 Reproduzierbarkeit der Druckverteilung und Grenzschichtrandströmung . . . . . . . . . . . . . 101

6.1.4 Grenzschichtgrundströmung . . . . . . . . . . . . 102

6.2 Lineare Stabilitätseigenschaften . . . . . . . . . . . . . . . . . . 104

6.2.1 Stationäre primäre Instabilitäten . . . . . . . . . . . . 104

6.2 .2 Instationäre primäre Instabilitäten . . . . . . . . . . . . 106

6.3 Transitionsszenario im neu ausgelegten Prinzipexperiment . . . 108

6.3.1 Stationäre Störströmung . . . . . . . . . . . . . . 108

6.3.2 Instationäre Störströmung . . . . . . . . . . . . . . . 114

6.3.3 Spannweitig und zeitlich gemittelte

Grenzschichtströmung im fortgeschrittenen Stadium . . . 117

6.3.4 Sekundärinstabilität und finaler Umschlag . . . . . . . . 119

6.3.5 Die verschiedenen Störungen im Überblick . . . . . . . . 125

7 Beeinflussung durch spannweitig periodische Wandheizung 127

7.1 Oberflächenbeschaffenheit des Heizaktuators . . . . . . . . . . 128

7.2 Die Störströmung stromab des ausgeschalteten Heizaktuators . 130

7.2.1 Überblick über die Störungsentwicklung . . . . . . . . . . 131

7.2.2 Instationäre Störungen im frühen Stadium . . . . . . . . 135 
7.2.3 Hochfrequente instationäre Störungen und Sekundärinstabilität . . . . . . . . . . . . . . . 140

7.3 Erwartete Temperaturänderungen aufgrund der Wandheizung . 145 7.3.1 Anhebung der spannweitig gemittelten Wandtemperatur 145

7.3.2 Erzeugte Wandtemperaturperiodizität . . . . . . . . . . . 150

7.3.3 Einfluss der Wandtemperaturperiodizität auf Hitzdrahtmessungen . . . . . . . . . . . . . . . 154

7.3.4 Einfluss der spannweitig gemittelten Heizung auf die lineare Stabilität . . . . . . . . . . . . . . . . 157

7.4 Systematische Variation der Heizleistung . . . . . . . . . . 160 7.4.1 Künstliche Anregung der stationären Kontrollmode . . . 161 7.4.2 Nichtlineare Wechselwirkungen stationärer Störungen . . 171

7.4.3 Zusätzlich angehobene instationäre Störungen . . . . . . 174

7.5 Transitionsszenario im heizaktuierten Fall . . . . . . . . . . . . 175

7.5.1 Die stationäre Störströmung . . . . . . . . . . . 176

7.5.2 Entwicklung stationärer Störungen . . . . . . . . . . 178

7.5.3 Entwicklung instationärer Störungen . . . . . . . . . . . 181

7.5.4 Sekundärinstabilitäten . . . . . . . . . . . 183

7.5.5 Überprüfung eines Transitionskriteriums . . . . . . . . . 187

8 Beeinflussung durch spannweitig periodische Rotation 191

8.1 Systematische Variation der Anregungsamplitude . . . . . . . . 192

8.1.1 Künstliche Anregung stationärer QSI: verteilte Anregung 193

8.1.2 Stationäre Grenzschichtdeformation durch Einzelanregung 200

8.1.3 Instationäre Störungen stromab der Einzelanregung . . . 204

8.2 Transitionsszenario im Fall mit Rotationsaktuierung . . . . . . 215

9 Zusammenfassung und Ausblick 221

9.1 Zusammenfassung . . . . . . . . . . . . . . . . . 221

9.2 Ausblick . . . . . . . . . . . . . . . . . . . . . . . . . . 227

Anhang $\quad 229$

A.1 Positionen der Druckbohrungen . . . . . . . . . . . . . . . 231

A.2 Strömungsfeld bei $x_{c} / c=0.8$ im Referenzfall . . . . . . . . . 232

A.3 Rotations-Einzelanregung: Instationäre Störungen bei $f=f_{\text {rot }} 233$

A.4 Rotations-Einzelanregung: Überblick über instationäre Störungen235

A.5 Drehteller ohne signifikante Anhebung instationärer Störungen 237 


\section{Nomenklatur}

\section{Römische Symbole}

A Parameter im Kingschen Gesetz

$A_{\text {ges }} \quad$ Kombinierte Störungsamplitude gemäß Borodulin et al. [13]

$A_{\text {instat }} \quad$ Bilanzierende Amplitude instationärer Störungen

$A_{\text {stat }} \quad$ Bilanzierende Amplitude stationärer Störungen

$A_{1}$

Querschnittsfläche der Beruhigungskammer des Hitzdrahtkalibrators

$A_{2} \quad$ Querschnittsfläche der Düse des Hitzdrahtkalibrators

$a_{s} \quad a_{s}\left(y_{c}\right):=u_{s}\left(y_{c}\right)-u_{s, 0}\left(y_{c}\right)$, isolierte Aktuierungswirkung

$a_{i j} \quad$ Richtungskoeffizient $j$ des Drahtes $i$

$B \quad$ Parameter im Kingschen Gesetz

c Profiltiefe der Modellplatte, $c=600 \mathrm{~mm}$

$c_{i} \quad$ Geschwindigkeitskoeffizienten bei getrennter Kalibrierung von Geschwindigkeits- und Richtungsabhängigkeit, $i=0,1,2,3,4$

$c_{p} \quad$ Druckbeiwert

$d \quad$ Durchmesser der Drehteller des Rotationsaktuators, $d=2 \mathrm{~mm}$

$d_{1} \quad$ Durchmesser der Beruhigungskammer des Hitzdrahtkalibrators

$d_{2} \quad$ Durchmesser der Düse des Hitzdrahtkalibrators

$d_{i}$

$E$

Koeffizienten in der quadratischen Gleichung 5.1.17, $i=1,2,3$

$E_{1}, E_{2}$

Anemometerspannung

$E_{t}$

Anemometerspannung zu Draht 1 bzw. 2

f Frequenz

Kinetische Energie in einer turbulenten Strömung

$f_{\text {rot }}$

Drehzahl bzw. Rotationsfrequenz der Drehteller im Rotationsaktuator

$g \quad$ Erdbeschleunigung $g=9.81 \mathrm{~m} / \mathrm{s}^{2}$

$H_{12} \quad$ Formfaktor der Grenzschicht

$k \quad$ Wellenzahl im räumlichen Turbulenzspektrum 
$\vec{k}$

$k_{\text {cal }}$

N

$n$

$P$

$p$

$\bar{p}$

$\widetilde{p}$

$p_{W}$

$\stackrel{\overrightarrow{\vec{q}}}{\vec{q}}$

$q$

$\vec{q}$

$q_{\infty}$

$q_{e}$

$q_{K, \infty}$

Re

$s$

$t$

$\mathrm{Tu}$

$T_{f}$

$U$

$U_{2}$

$u_{c}$

$U_{k}$

$u_{c, \infty}$

$u_{c, e}$

$u_{S o}$

$v_{c}$

$v_{c, \infty}$

$v_{c, e}$

$v_{S o}$

$w$

$\vec{w}$

$w_{\text {ind }}$

$x_{c}$

$x_{s}$
Wellenvektor einer Störmode gemäß Normalmodenansatz

Düsenfaktor des Hitzdrahtkalibrators

N-Faktor, integrale Anfachung einer Störmode gemäß Normalmodenansatz, $N=\int \sigma d x_{c}$

Parameter im Kingschen Gesetz

Heizleistung

Statischer Druck

Statischer Druck in der Grundströmung

Statischer Druck in der Störströmung

Statischer Druck an der Wand

Geschwindigkeitsvektor der stationären Grundströmung

Geschwindigkeitsvektor der Störströmung

Geschwindigkeitsvektor

Betrag der Anströmgeschwindigkeit

Betrag der Grenzschichtrandgeschwindigkeit, $q_{e}=\sqrt{u_{c, e}^{2}+v_{c, e}^{2}}$

Anströmgeschwindigkeit wie mit der Windkanalmesstechnik bestimmt

Reynoldszahl

Standardabweichung

Zeit

Turbulenzgrad der Anströmung

Filmtemperatur, zur Definition siehe Abschnitt 5.1.1

Gesamtbetrag der Anströmgeschwindigkeit

Ausströmungsgeschwindigkeit des Hitzdrahtkalibrators

Geschwindigkeitskomponente in $x_{c}$-Richtung

Normierte Kühlgeschwindigkeit des Hitzdrahtes

$u_{c}$ in der ungestörten Anströmung

$u_{c}$ am Grenzschichtrand

Geschwindigkeitskomponente in Richtung der Sondenachse

Geschwindigkeitskomponente in $y_{c}$-Richtung

$v_{c}$ in der ungestörten Anströmung

$v_{c}$ am Grenzschichtrand

Geschwindigkeitskomponente senkrecht zur Sondenachse

Wandnormale Geschwindigkeitskomponente

Wirbelvektor

Induzierte wandnormale Geschwindigkeit

Koordinate im modellfesten Koordinatensystem (in Profiltiefenrichtung)

Koordinate in Hauptströmungsrichtung des potentialstromlinienorientierten Koordinatensystems 


\begin{tabular}{|c|c|}
\hline$x_{T}$ & X-Achse im Koordinatensystem der Traversiereinrichtung \\
\hline$y_{c}$ & $\begin{array}{l}\text { Koordinate im modellfesten Koordinatensystem (spannweitige } \\
\text { Koordinate) }\end{array}$ \\
\hline$y_{s}$ & $\begin{array}{l}\text { Koordinate senkrecht zur gekrümmten Potentialstromlinie, po- } \\
\text { sitiv in Richtung des Krümmungsmittelpunkts }\end{array}$ \\
\hline$y_{T}$ & Y-Achse im Koordinatensystem der Traversiereinrichtung \\
\hline & Wandabstand \\
\hline$z_{T}$ & Z-Achse im Koordinatensystem der Traversiereinrichtung \\
\hline \multicolumn{2}{|c|}{ Griechische Symbole } \\
\hline$\alpha^{\prime}$ & $\begin{array}{l}\text { Erste (komplexwertige) Komponente des Wellenvektors } \vec{k} \text { einer } \\
\text { Störmode gemäß Normalmodenansatz }\end{array}$ \\
\hline$\alpha$ & $\begin{array}{l}\text { Realteil von } \alpha^{\prime} \text {, Wellenzahl der Periodizität einer Störmode in } \\
x_{c} \text {-Richtung }\end{array}$ \\
\hline$\beta^{\prime}$ & $\begin{array}{l}\text { Zweite (komplexwertige) Komponente des Wellenvektors } \vec{k} \\
\text { einer Störmode gemäß Normalmodenansatz }\end{array}$ \\
\hline$\beta$ & $\begin{array}{l}\text { Realteil von } \beta^{\prime} \text {, Wellenzahl der Periodizität einer Störmode in } \\
y_{c} \text {-Richtung }\end{array}$ \\
\hline$\gamma$ & In der Numerik verwendeter Anstellwinkel der Konfiguration \\
\hline$\delta x$ & Größe eines elementaren Schrittes der jeweiligen Achse $x$ \\
\hline$\delta_{1}$ & Verdrängungsdicke \\
\hline$\delta_{2}$ & Impulsverlustdicke \\
\hline$\delta_{99}$ & $\begin{array}{l}\text { Grenzschichtdicke, definiert als der Wandabstand, an dem der } \\
\text { Betrag der Geschwindigkeit } 99 \% \text { des Betrags der Grenzschicht- } \\
\text { randgeschwindigkeit entspricht. }\end{array}$ \\
\hline$\epsilon_{a}$ & Unsicherheit der Größe $a$ \\
\hline$\eta$ & Kontraktionsverhältnis des Hitzdrahtkalibrators \\
\hline$\kappa$ & Adiabatenexponent von Luft \\
\hline$\Lambda$ & Wärmeleitfähigkeit von Luft \\
\hline$\lambda$ & Spannweitige Wellenlänge \\
\hline$\mu$ & Dynamische Viskosität \\
\hline$\nu$ & Kinematische Viskosität \\
\hline$\xi$ & $\begin{array}{l}\text { Materialspezifischer Temperaturkoeffizient des Hitzdrahtwider- } \\
\text { stands }\end{array}$ \\
\hline$\rho$ & Dichte \\
\hline$\sigma$ & $\begin{array}{l}\text { Negativer Imaginärteil von } \alpha^{\prime} \text {, Anfachungsrate einer Störmode } \\
\text { entlang } x_{c} \text { gemäß Normalmodenansatz }\end{array}$ \\
\hline$\tau_{x z}$ & $\begin{array}{l}\text { Wandschubspannung: Spannung, welche an der Fläche angreift, } \\
\text { deren Normalenvektor in } z \text {-Richtung zeigt und in } x \text {-Richtung } \\
\text { wirkt }\end{array}$ \\
\hline & Schiebewinkel der ungestörten Anströmung \\
\hline
\end{tabular}




$\begin{array}{ll}\phi_{T} & \text { Rotationswinkel der Traversiereinrichtung } \\ \phi_{g, \infty} & \text { Geometrischer Schiebewinkel des Modells } \\ \phi_{S o} & \text { Sondenschiebewinkel } \\ \chi & \text { Überhitzungsverhältnis des Hitzdrahtes } \\ \omega & \text { Kreisfrequenz } \omega=2 \pi f\end{array}$

\section{Indizes}

$\infty$

0

c

e

ind

K

rms

$\mathrm{s}$

So

$\mathrm{T}$

W
Wert in der ungestörten Anströmung

Anfangsbedingung

Wert im plattenfesten Koordinatensystem

Wert am Grenzschichtrand

Induzierte Größe

Auf den Windkanal bezogen

Effektivwert einer periodischen Strömungsgröße

Wert im stromlinienorientierten Koordinatensystem

Wert im sondenfesten Koordinatensystem

Wert im Koordinatensystem der Traversiereinrichtung

Wert an der Wand

\section{Hochgestellte Zeichen}

$\bar{a}$

$\hat{a}$

$\hat{a}^{*}$

$\widetilde{a}$

$a^{\prime}$

Kurzschreibweise für den Mittelwert der Größe $a: \bar{a}=\operatorname{mean}_{b}(a)$, wobei $b$ jeweils im Kontext definiert wird

Amplitude einer spannweitigen Periodizität der Größe $a$

Amplitude einer örtlich lokalisierten stationären Einzelstörung,

Definition siehe Abschnitt 8.1.1

Störanteil der Strömungsgröße $a$ der Grenzschichtströmung

Amplitude einer zeitlichen Periodizität in $a$

\section{Operatoren}

$|a|$

$\Im(a)$

$\Re(a)$

$\Delta a$

$\max _{b}(a)$

$\operatorname{mean}_{b}(a)$
Betrag der Größe $a$

Imaginärteil der Größe $a$

Realteil der Größe $a$

Differenzbetrag der Größe $a$

Das Maximum der Zielgröße $a$ entlang der Koordinate oder Einflussgröße $b$

Der Mittelwert der Zielgröße $a$ entlang der Koordinate oder

Einflussgröße $b$

\section{Abkürzungen}

$1 \mathrm{MG}$

$\mathrm{CNC}$
Ein-Meter-Windkanal Göttingen

Computerized Numerical Control, rechnergestützte numerische Steuerung 
DLR Deutsches Zentrum für Luft- und Raumfahrt e.V.

DNS Direkte Numerische Simulation

DRE Discrete Roughness Element (diskretes Rauigkeitselement, im Gegensatz zu verteilter Rauigkeit)

LST Lineare Stabilitätstheorie

MFD Mean Flow Distortion, Deformation der spannweitig gemittelten Grenzschichtströmung

SAMS Software für automatische Messdatenerfassung und Schrittmotorsteuerung

TS Tollmien-Schlichting

UFD Upstream Flow Deformation

\section{Angabe von Unsicherheiten}

Bei der Angabe von Messwerten oder daraus abgeleiteten Werten wird zur Angabe der jeweiligen Unsicherheit die sog. Klammerschreibweise verwendet (im englischen Sprachgebrauch ,concise notation“). Dabei wird die Unsicherheit der letzten angegebenen Dezimalstelle in runden Klammern hinter dem Wert angegeben.

Beispiele:

$25.75(7) \mathrm{m} / \mathrm{s}=25.75 \mathrm{~m} / \mathrm{s} \pm 0.07 \mathrm{~m} / \mathrm{s}$

Letzte angegebene Dezimalstelle: $10^{-2} \mathrm{~m} / \mathrm{s}$, die Angabe „(7)“ beschreibt eine Unsicherheit von $7 \cdot 10^{-2} \mathrm{~m} / \mathrm{s}$.

$301(2) \mathrm{K}=301 \mathrm{~K} \pm 2 \mathrm{~K}$

Letzte angegebene Dezimalstelle: $10^{0} \mathrm{~K}$, die Angabe „(2)" beschreibt eine Unsicherheit von $2 \cdot 10^{0} \mathrm{~K}=2 \mathrm{~K}$. 


\section{Kapitel 1}

\section{Einleitung}

\section{$1.1 \quad$ Einführung}

Der Reibungswiderstand einer laminaren Grenzschicht ist geringer als der einer turbulenten Grenzschicht. Es ist daher besonders in Fällen, in denen der Reibungswiderstand einen signifikanten Teil des Gesamtwiderstands ausmacht, von großem Interesse, den laminar-turbulenten Umschlag zu verzögern und die laminare Lauflänge des umströmten Körpers zu vergrößern. Bei Flugzeugen kann so Treibstoff gespart und es können somit Kosten und Klimaemissionen reduziert werden. Bei der Erforschung des laminar-turbulenten Umschlags, im Folgenden auch Transition genannt, wurden verschiedene Möglichkeiten zur Laminarhaltung von Grenzschichtströmungen identifiziert. Passive Maßnahmen greifen dabei vor allem bei der Formgebung des umströmten Körpers an, um die Stabilität der Grenzschicht zu erhöhen oder um das anfängliche Störungsniveau zu verringern. Aktive Methoden greifen dagegen mit einem gewissen Energieaufwand störungsreduzierend in der Grenzschicht ein.

Besondere technische Bedeutung kommt der Transition an gepfeilten Flügeln zu. Diese werden üblicherweise für transsonische Reisegeschwindigkeiten eingesetzt, da die Kompressibilitätseffekte in erster Linie durch die Geschwindigkeitskomponente normal zur Flügelvorderkante bestimmt werden. Durch die Zerlegung der Anströmgeschwindigkeit in eine Komponente normal und eine Komponente tangential zur Vorderkante werden die Kompressibilitäts- 
effekte abgeschwächt. Die tangentiale Komponente führt aber zu einer zusätzlichen Querströmung, die zu grundlegend anderen Prozessen im laminarturbulenten Umschlag führen kann als in einer nicht schiebend angeströmten Grenzschicht. Dominieren diese für schiebend angeströmte Grenzschichten spezifischen Prozesse das Szenario, spricht man vom querströmungsdominierten Transitionsprozess, welcher näher in Abschnitt 2.3 beschrieben wird.

Für die querströmungsdominierte Transition wurde neben direkt stabilisierenden passiven oder störungsreduzierenden aktiven Laminarhaltungsmethoden eine Methode identifiziert, welche durch zusätzlich eingebrachte Störungen auf indirekte Weise eine Verzögerung des Umschlags ermöglicht [95]. Diese Methode wird als Upstream Flow Deformation (UFD) bezeichnet [112] und wird in Abschnitt 2.4 beschrieben. Sie ist insbesondere deshalb von wissenschaftlichem Interesse, weil sie durch ihre indirekte Funktionsweise das Potential besitzt, im Vergleich zu direkten Laminarhaltungsmethoden besonders effizient die Transition zu verzögern. Die indirekte Funktionsweise macht die Methode allerdings auch sehr empfindlich gegenüber den Eigenschaften und der Stärke der zusätzlich eingebrachten Störungen. Zusätzlich hängt die geeignete Wahl der Störungseigenschaften von den Anströmungsbedingungen ab. Es ist daher nötig, diese Eigenschaften bei der künstlichen Störungsanregung steuern zu können und idealerweise auch an veränderliche Anströmungsbedingungen anpassbar zu gestalten. Die Untersuchung von neuen flexiblen Anregungskonzepten für zusätzliche Störungen mit wohldefinierten Eigenschaften im Kontext dieser Laminarhaltungsmethode ist Hauptthema der vorliegenden Arbeit.

\subsection{Zielsetzung und Überblick}

Das Prinzipexperiment der schiebenden ebenen Platte, wie es vom Ende der 1980er bis Anfang der 2000er Jahre am DLR Göttingen zur fundamentalen Untersuchung des querströmungsdominierten Transitionsprozesses verwendet wurde, war für die Untersuchung von Aktuierungskonzepten im Kontext der UFD-Laminarhaltungsmethode nur bedingt geeignet und existierte zudem nur noch in Teilen. Daher ist ein grundlegender Teil der Zielsetzung im Rahmen dieser Arbeit, einen ähnlichen experimentellen Aufbau mit besser geeigneten Strömungseigenschaften neu auszulegen und aufzubauen. Der Prozess der Neuauslegung wird in Kapitel 4 näher motiviert, beschrieben und sein Ergebnis vorgestellt. 
Zur gezielten Anregung der zusätzlichen Störungen (stationäre Querströmungsinstabilitäten, QSI) in der UFD-Methode können beispielsweise zylindrische Einzelrauigkeiten verwendet werden [84, 97], welche spannweitig periodisch auf der Modelloberfläche fixiert werden. Die UFD-Methode ist sehr empfindlich gegenüber der Anfangsamplitude der zusätzlichen Störungen und erfordert neben einer wohldefinierten spannweitigen Wellenlänge eine wohldefinierte Anfangsamplitude der Störungen. Der geeignete Wertebereich für die spannweitige Wellenlänge und die Amplitude hängt von den Anströmungsbedingungen ab. Bei fester Reynoldszahl der Anströmung wird der gewünschte transitionsverzögernde Effekt nicht erzielt, wenn die Amplitude der zusätzlich angeregten Störung zu gering ist. Ist sie zu groß, kann die zusätzlich angeregte Störung selbst zu einer stromauf verlagerten Transition führen. Daher sind Anregungskonzepte mit regelbarer Amplitude von Interesse, man nennt sie auch aktive Rauigkeiten. Bisher wurden bereits zwei solcher Anregungskonzepte mit dem Ziel der Transitionsverzögerung anhand der UFD-Methode untersucht: die Anregung durch spannweitig periodische pneumatische Aktuierung [68] und die Anregung durch spannweitig periodische Plasmaaktuierung (siehe z.B. [105]). Mithilfe der pneumatischen Aktuierung konnte dabei eine Transitionsverzögerung experimentell nachgewiesen werden, mit der Plasmaaktuierung bisher nicht.

Im Rahmen dieser Arbeit wurden nun zwei weitere solcher Anregungskonzepte, die Anregung durch spannweitig periodische Wandheizung und die Anregung durch spannweitig periodisch angeordnete rotierende Scheiben technisch realisiert und im neu ausgelegten Prinzipexperiment untersucht. Hypothesen zur bisher teilweise ungeklärten physikalischen Funktionsweise dieser Störungsanregung werden in Abschnitt 2.5 beschrieben. Die technische Realisierung der Aktuatoren wird in Abschnitt 4.5 beschrieben.

Ziel der Arbeit ist es, die Funktion der bislang im Falle der Oberflächenheizung wenig und im Falle der Rotationsanregung gar nicht untersuchten Anregungskonzepte zu untersuchen und ihre Eignung für einen Einsatz in der UFD-Methode zu überprüfen. Dazu ist zu zeigen, dass es mit beiden Anregungskonzepten möglich ist, die Amplitude einer zusätzlichen wohldefinierten Störung zu kontrollieren und dies anhand eines charakteristischen Parameters für die Anregungsstärke zu charakterisieren. Es ist des Weiteren auch von besonderem Interesse, inwiefern die Anregungskonzepte in der Lage sind, die beabsichtigte Störung isoliert von anderen Störungen anzuregen, da dies bspw. bei der Plasmaaktuierung bisher nicht der Fall war. Des Weiteren soll 
überprüft werden, ob eine Transitionsverzögerung mithilfe der untersuchten Anregungskonzepte erreicht wird.

Der theoretische Hintergrund für die Interpretation der experimentellen Ergebnisse und der Stand der Forschung zur Verzögerung der querströmungsdominierten Transition werden in Kapitel 2 eingeführt. Die numerischen und experimentellen Methoden werden in Kapitel 3 bzw. 5 beschrieben, wobei der Fokus der Arbeit klar auf der experimentellen Untersuchung mithilfe von Hitzdrahtanemometrie liegt, deren Interpretation durch numerische Untersuchungen unterstützt wird.

Den Hauptteil der Arbeit bilden die Kapitel 6, 7 und 8 mit experimentellen Ergebnissen aus der Grenzschicht des neu ausgelegten Prinzipexperiments. Zunächst wird in Kapitel 6 geprüft, inwiefern die realisierte Grenzschichtströmung im neu ausgelegten Prinzipexperiment die Ziele der Neuauslegung erfüllt und sich für Untersuchungen zur UFD-Methode eignet. Dazu wird in Abschnitt 6.1 im Wesentlichen gezeigt, dass die Grenzschichtströmung erfolgreich durch eine numerische Simulation nachvollzogen werden kann. Basierend auf der numerisch simulierten Grenzschichtströmung werden in Abschnitt 6.2 Ergebnisse einer linearen lokalen Stabilitätsuntersuchung vorgestellt. Mithilfe der dabei gewonnenen Erkenntnisse über die in der Grenzschicht zu erwartenden Instabilitäten kann in Abschnitt 6.3 das gesamte Transitionsszenario der Grenzschicht inklusive der beobachteten Störströmung ohne Aktuierung nachvollzogen werden. Dies ist insbesondere für das Verständnis der späteren gezielten Beeinflussung der Störströmung durch die Aktuatoren wichtig.

Im Anschluss wird in Kapitel 7 untersucht, ob mithilfe der spannweitig periodischen Wandheizung stationäre QSI angeregt werden können, ob die Amplitude der angeregten QSI kontrollierbar ist und welchen Einfluss die Aktuierung auf das Transitionsszenario hat. Dazu muss wegen des besonderen Materials des Aktuators zunächst bewertet werden, inwiefern die Oberflächenbeschaffenheit die Störströmung bereits im nicht-aktuierten Fall verändert (Abschnitte 7.1 und 7.2). Des Weiteren muss in Abschnitt 7.3 diskutiert werden, inwiefern die Wärmezufuhr des Heizaktuators Hitzdrahtmessungen in der Grenzschicht und die Stabilität der Grenzschicht beeinflusst. Anhand einer systematischen Variation der Heizleistung wird in Abschnitt 7.4 gezeigt, dass erfolgreich stationäre QSI angeregt werden und dass ihre Amplitude in einem großen Bereich der Heizleistung einem eindeutigen Zusammenhang mit der Heizleistung folgt und somit kontrollierbar ist. Für ausgewählte Betriebsparameter wird in Abschnitt 7.5 gezeigt, welchen Einfluss die Heizaktuierung 
auf das Transitionsszenario inklusive der Störströmung hat und analysiert, ob anhand der UFD-Methode eine Transitionsverzögerung erreicht wird.

Die künstliche Anregung stationärer QSI durch spannweitig periodisch angeordnete, rotierende Scheiben wird in Kapitel 8 untersucht. Auch für diese Anregungsmethode kann anhand einer systematischen Variation der Rotationsfrequenz in Abschnitt 8.1 gezeigt werden, dass stationäre QSI erfolgreich angeregt werden und dass ihre Amplitude in einem eindeutigen Zusammenhang zur Rotationsfrequenz steht. Dabei wird auch geprüft, ob der Drehsinn der Scheiben einen wesentlichen Einfluss hat. Durch den alleinigen Betrieb einzelner rotierender Scheiben wird auch die stationäre Störung durch solch eine Einzelanregung untersucht und ein eindeutiger Zusammenhang ihrer Amplitude zur Rotationsfrequenz hergestellt. Stromab der Aktuierung werden signifikant angehobene instationäre Fluktuationen beobachtet. Es wird geprüft, ob es sich dabei um Folgen einer unvollkommenen technischen Realisierung oder um eine inhärente Eigenschaft der Anregung durch Rotation handelt. Für ausgewählte Betriebsparameter wird in Abschnitt 8.2 gezeigt, welchen Einfluss die spannweitig periodische Rotationsaktuierung auf das Transitionsszenario hat. 


\section{Kapitel 2}

\section{Hintergrund und Stand der Forschung}

Der Umschlag von einer laminaren Grenzschichtströmung zu einer turbulenten Grenzschichtströmung findet unter verschiedenen Bedingungen über unterschiedliche Mechanismen statt, welche teilweise stark unterschiedliche oder gar gegensätzliche Eigenschaften aufweisen. Um den Transitionsprozess $\mathrm{zu}$ verstehen und letztendlich gezielt beeinflussen zu können, müssen diese Mechanismen und ihre beherrschenden Parameter getrennt betrachtet werden. In dieser Arbeit wird der querströmungsdominierte Transitionsprozess einer inkompressiblen Grenzschicht betrachtet. Er kann dann auftreten, wenn die Grenzschichtströmung zusätzlich zur Geschwindigkeitskomponente in Hauptströmungsrichtung eine nicht verschwindende Querkomponente besitzt, was unter anderem bei schiebend angeströmten Tragflügeln der Fall ist. Man spricht bei solchen Grenzschichten von dreidimensionalen (3D-)Grenzschichten.

In Abschnitt 2.1 werden zuerst die Annahmen für die theoretische Behandlung der im Rahmen dieser Arbeit untersuchten Grundströmung dargestellt. Daraufhin werden in Abschnitt 2.2 die Grundlagen der theoretischen Beschreibung der Instabilitäten in einer solchen Grundströmung beschrieben. In den folgenden Abschnitten werden dann die bisherigen Erkenntnisse zu den relevanten physikalischen Prozessen zusammengefasst. 


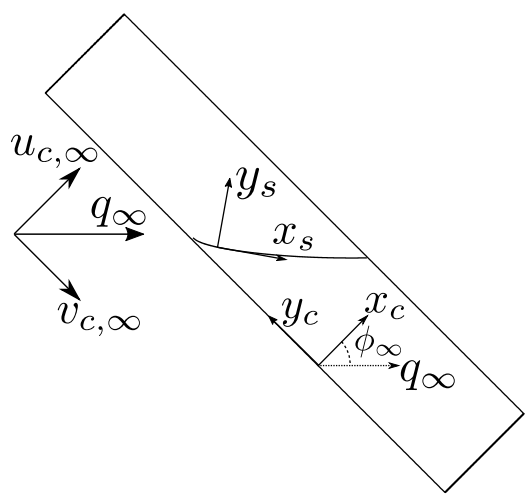

Abbildung 2.1.1: Die verwendeten Koordinatensysteme in einer Aufsichtsskizze der schiebenden ebenen Platte

\subsection{Die Grundströmung}

Um die relevanten Eigenschaften des querströmungsdominierten Transitionsprozesses theoretisch zu behandeln, betrachtet man idealisiert eine unendlich schiebende Grundströmung an einer ebenen Platte. Damit ist eine quasidreidimensionale Strömungssituation gemeint, in der alle spannweitigen Gradienten vernachlässigt werden können. Gegenüber einem realen schiebend angeströmten Flügel ist dies nur eine Annäherung, unter anderem da an einem endlichen Flügel am jeweiligen spannweitigen Ende ein Druckausgleich zwischen der Ober- und Unterseite des Flügels stattfindet, was zwangsweise zu spannweitigen Druck- und Geschwindigkeitsgradienten führt.

Im Folgenden und im weiteren Verlauf der Arbeit werden zwei Koordinatensysteme verwendet, das modellorientierte Koordinatensystem mit den Koordinaten $x_{c}$ und $y_{c}$ und das stromlinienorientierte Koordinatensystem mit den Koordinaten $x_{s}$ und $y_{s}$. Letzeres orientiert sich an der gekrümmten Grenzschichtrandstromlinie. In beiden Koordinatensystemen bezeichnet $z$ den Wandabstand. Die beiden Koordinatensysteme sind in Abb. 2.1.1 dargestellt. Da es sich bei dem umströmten Modell, an dessen Oberfläche die Grenzschicht untersucht wird, um eine Modellplatte handelt (siehe Kapitel 4), werden im Folgenden auch die Begriffe plattenorientiert, plattenparallel und für $x_{c}$ der Begriff Plattentiefenposition verwendet. 
Projiziert man den Betrag der Anströmgeschwindigkeit $q_{\infty}$ in das Plattenkoordinatensystem, erhält man die Komponenten $u_{c, \infty}$ und $v_{c, \infty}$ der Anströmung.

$$
\begin{aligned}
& u_{c, \infty}=q_{\infty} \cdot \cos \left(\phi_{\infty}\right) \\
& v_{c, \infty}=q_{\infty} \cdot \sin \left(\phi_{\infty}\right)
\end{aligned}
$$

Dabei entspricht $\phi_{\infty}$ dem geometrischen Schiebewinkel der Modellplatte $\phi_{g, \infty}$. Die oben beschriebenen Annahmen zur unendlich schiebenden Strömung lassen sich wie folgt ausdrücken:

$$
\begin{aligned}
\frac{\partial u_{c}}{\partial y_{c}}=\frac{\partial v_{c}}{\partial y_{c}}=\frac{\partial w_{c}}{\partial y_{c}} & =0 \\
\frac{\partial p}{\partial y_{c}} & =0
\end{aligned}
$$

Dabei bezeichnet $p$ den statischen Druck. Die dreidimensionalen stationären und inkompressiblen Grenzschichtgleichungen erster Ordnung mit konstanter kinematischer Viskosität $\nu$ und Dichte $\rho$ lauten (siehe [100]):

$$
\begin{aligned}
& u_{c} \frac{\partial u_{c}}{\partial x_{c}}+v_{c} \frac{\partial u_{c}}{\partial y_{c}}+w \frac{\partial u_{c}}{\partial z}=-\frac{1}{\rho} \frac{\partial p}{\partial x_{c}}+\nu \frac{\partial^{2} u_{c}}{\partial z^{2}} \\
& u_{c} \frac{\partial v_{c}}{\partial x_{c}}+v_{c} \frac{\partial v_{c}}{\partial y_{c}}+w \frac{\partial v_{c}}{\partial z}=-\frac{1}{\rho} \frac{\partial p}{\partial y_{c}}+\nu \frac{\partial^{2} v_{c}}{\partial z^{2}} \\
& \vec{\nabla} \vec{q}=\frac{\partial u_{c}}{\partial x_{c}}+\frac{\partial v_{c}}{\partial y_{c}}+\frac{\partial w}{\partial z}=0
\end{aligned}
$$

Dabei bezeichnet $w$ die wandnormale Geschwindigkeitskomponente und $\vec{q}$ den Geschwindigkeitsvektor. Mit den Annahmen 2.1.3 und 2.1.4 vereinfachen sie sich zu:

$$
\begin{aligned}
u_{c} \frac{\partial u_{c}}{\partial x_{c}}+w \frac{\partial u_{c}}{\partial z} & =-\frac{1}{\rho} \frac{\partial p}{\partial x_{c}}+\nu \frac{\partial^{2} u_{c}}{\partial z^{2}} \\
u_{c} \frac{\partial v_{c}}{\partial x_{c}}+w \frac{\partial v_{c}}{\partial z} & =\nu \frac{\partial^{2} v_{c}}{\partial z^{2}} \\
\frac{\partial u_{c}}{\partial x_{c}}+\frac{\partial w}{\partial z} & =0
\end{aligned}
$$

Am Grenzschichtrand $\left(z=\delta\left(x_{c}\right)\right.$ mit der Grenzschichtdicke $\delta$ ) geht die Strömung in die reibungsfrei anzunehmende Außenströmung über und nach Grenzschichttheorie erster Ordnung verschwinden die wandnormalen Gradienten:

$$
\frac{\partial u_{c, e}}{\partial z}=\frac{\partial v_{c, e}}{\partial z}=\frac{\partial w_{e}}{\partial z}=0
$$


Dabei bezeichnet der Index e die Strömungsgrößen am Grenzschichtrand. In der Herleitung der Grenzschichtgleichungen erster Ordnung ergibt sich aus der Abschätzung der Größenordnungen, dass in der Grenzschicht $\frac{\partial p}{\partial z} \approx 0$ gilt. Man spricht davon, dass der Druck in der Grenzschicht von der reibungsfreien Außenströmung aufgeprägt wird. Daher wird hier nicht zwischen $p$ und $p_{e}$ unterschieden. Man erhält aus Gleichung 2.1.8 die Beziehung:

$$
u_{c, e} \frac{\partial u_{c, e}}{\partial x_{c}}=-\frac{1}{\rho} \frac{\partial p}{\partial x_{c}}
$$

Die Druckverteilung $p\left(x_{c}\right)$ bestimmt also die Verteilung $u_{c, e}\left(x_{c}\right)$ am Grenzschichtrand. Durch Integration entlang der reibungslosen Grenzschichtrandstromlinie aus der unendlichen Anströmung (mit Druck $p_{\infty}$ und Geschwindigkeit $\left.u_{c, \infty}\right)$ bis zur Position $x_{c}$ erhält man:

$$
c_{p}:=\frac{p\left(x_{c}\right)-p_{\infty}}{\frac{1}{2} \rho u_{c, \infty}^{2}}=1-\left(\frac{u_{c, e}\left(x_{c}\right)}{u_{c, \infty}}\right)^{2}
$$

Durch den sehr kleinen wandnormalen Druckgradienten in der Grenzschicht entspricht der Druck $p\left(x_{c}\right)$ nahezu dem mit Druckbohrungen an der Wand messbaren Druck $p_{W}\left(x_{c}\right)$. Gleichsam wird aus Gleichung 2.1.9 am Grenzschichtrand folgende Aussage:

$$
u_{c, e} \frac{\partial v_{c, e}}{\partial x_{c}}=0
$$

Da am Grenzschichtrand $\left|u_{c, e}\right|>0$ gilt, ergibt sich

$$
\frac{\partial v_{c, e}}{\partial x_{c}}=0 \rightarrow v_{c, e}=\text { const. }=v_{c, \infty} .
$$

Die spannweitige Komponente der ungestörten Anströmung behält also am Grenzschichtrand ihren konstanten Wert entlang $x_{c}$ bei. Im Rest der Arbeit wird oft der Betrag der Grenzschichtrandgeschwindigkeit $q_{e}=\sqrt{u_{c, e}^{2}+v_{c, e}^{2}}$ als Bezugsgröße verwendet werden.

\section{$2.2 \quad$ Lineare Stabilitätstheorie}

Bei der Erforschung des laminar-turbulenten Umschlags wurde schnell klar, dass kleine anfängliche Störungen in der Grenzschicht eine wichtige Rolle spielen. Für die Arbeiten der frühen Zeit der Stabilitätstheorie sei vor allem 
auf Reynolds [91] und Rayleigh [85] verwiesen. Zur theoretischen Behandlung des Transitionsprozesses im Rahmen der linearen Stabilitätstheorie wird die Strömung in eine Grundströmung und eine Störströmung aufgeteilt. Dabei wird die Annahme getroffen, dass in einer laminaren Grenzschicht den stationären Geschwindigkeitskomponenten $\bar{u}, \bar{v}$ und $\bar{w}$ verhältnismäßig kleine Störanteile $\widetilde{u}, \widetilde{v}$ und $\widetilde{w}$ überlagert sind. Eine Störströmung entsteht durch eine Anfangsanregung, welche stromab in ihrer Amplitude anwachsen oder abnehmen kann. Dieses Verhalten hängt von den Eigenschaften der betrachteten Grenzschicht ab. Wird eine Störung von der betrachteten Grenzschicht angefacht und nimmt ihre Amplitude zu, ist die Grenzschicht instabil gegenüber dieser Störung. Ist die Störung hingegen durch die Grenzschicht gedämpft und ihre Amplitude nimmt ab, ist die Grenzschicht gegenüber dieser Störung stabil. Einen Überblick über die lineare Stabilitätstheorie geben bspw. Reed et al. [86]. Die Beschreibung in diesem Abschnitt beschränkt sich auf den Umfang, der für die Diskussion der experimentell beobachteten Auswirkungen von Instabilitätsmoden nötig ist.

Für die Herleitung der Stördifferentialgleichungen geht man für den hier betrachteten Fall von den inkompressiblen Navier-Stokes-Gleichungen aus, welche in dimensionsloser Vektordarstellung wie folgt lauten:

$$
\frac{\partial \vec{q}}{\partial t}+(\vec{q} \cdot \vec{\nabla}) \vec{q}=-\vec{\nabla} p+\frac{1}{R e} \Delta \vec{q}
$$

mit der inkompressiblen Divergenzfreiheit:

$$
\vec{\nabla} \cdot \vec{q}=0
$$

Wie oben beschrieben, nimmt man an, dass der stationären Grundströmung $\overrightarrow{\vec{q}}$ eine Störströmung $\overrightarrow{\widetilde{q}}$ überlagert ist, zu der auch ein Druckanteil gehört:

$$
\begin{aligned}
& \vec{q}=\overrightarrow{\vec{q}}+\overrightarrow{\widetilde{q}} \\
& p=\bar{p}+\widetilde{p}
\end{aligned}
$$

Man nimmt des Weiteren an, dass sowohl die stationäre Grundströmung als auch die Summe aus Grundströmung und Störströmung die Navier-StokesGleichungen erfüllen. Setzt man Gleichungen 2.2.3 und 2.2.4 in die NavierStokes-Gleichungen 2.2.1 ein und vernachlässigt nichtlineare Terme, erhält man die linearisierten Stördifferentialgleichungen:

$$
\begin{aligned}
\frac{\partial \overrightarrow{\widetilde{q}}}{\partial t}+(\overrightarrow{\vec{q}} \cdot \vec{\nabla}) \overrightarrow{\widetilde{q}}+(\overrightarrow{\vec{q}} \cdot \vec{\nabla}) \overrightarrow{\tilde{q}} & =-\vec{\nabla} \widetilde{p}+\frac{1}{R e} \Delta \overrightarrow{\vec{q}} \\
\vec{\nabla} \cdot \overrightarrow{\widetilde{q}} & =0
\end{aligned}
$$


Die Stabilitätsuntersuchungen im Rahmen dieser Arbeit beschränken sich auf eine weitere Vereinfachung dieser Gleichungen, die lokale lineare Stabilitätsanalyse, welche oft mit „LST" (-Analyse) abgekürzt wird. Dazu wird die sog. Parallelströmungsannahme getroffen, welche von einer Grundströmung ausgeht, die nur vom Wandabstand $z$ abhängt. Änderungen der Grundströmung in $x_{c^{-}}$und $y_{c}$-Richtung werden vernachlässigt und es wird angenommen, dass die wandnormale Geschwindigkeitskomponente verschwindet $(\bar{w}=0)$. Diese Annäherung bedeutet, dass die Stabilität der Grenzschichtströmung an einer bestimmten Position $x_{c}$ nur durch die dort lokalen Bedingungen bestimmt ist. Dann kann der Geschwindigkeitsanteil der Störungen mit folgendem Lösungsansatz dargestellt werden:

$$
\overrightarrow{\widetilde{q}}\left(x_{c}, y_{c}, z, t\right)=\overrightarrow{\hat{q}}(z) \cdot e^{i\left(\alpha^{\prime} x_{c}+\beta^{\prime} y_{c}-\omega t\right)}+c . c .
$$

Dabei bezeichnet c.c. das komplex Konjugierte des ersten Termes, da die physikalische Störströmung reellwertig sein muss. Man betrachtet also die Störströmung als eine räumlich und zeitlich periodische Welle. Ihre Amplitude und wandnormale Struktur wird im Störansatz durch die Amplitudenfunktion $\overrightarrow{\hat{q}}=\overrightarrow{\hat{q}}(z)=\left(\hat{u}_{c}, \hat{v}_{c}, \hat{w}_{c}\right)^{T}$ beschrieben. Mit diesem Ansatz lassen sich die linearisierten Störgleichungen 2.2.5 und 2.2.6 mit geeigneten Randbedingungen als Eigenwertproblem formulieren. Die Randbedingungen sehen eine verschwindende Störströmung sowohl an der Wand als auch in großem Wandabstand vor. Im sog. räumlichen Problem ist die Kreisfrequenz $\omega=2 \pi f$ rein reellwertig, während $\alpha^{\prime}$ und $\beta^{\prime}$ im Störansatz komplexwertig sind. Der Imaginärteil beschreibt jeweils das Wachstum der Störmode, welches im räumlichen Problem räumlich verläuft. In dem hier betrachteten Fall unendlich schiebender Bedingungen verschwindet aber der Imaginärteil von $\beta^{\prime}\left(\Im\left(\beta^{\prime}\right)=0\right)$ aufgrund der spannweitig invarianten Grundströmung. Die Kreisfrequenz und die spannweitige Wellenzahl $\beta=\Re\left(\beta^{\prime}\right)$ werden reell vorgegeben und das komplexwertige $\alpha^{\prime}$ als Eigenwert ermittelt. Der Realteil von $\alpha^{\prime}$ entspricht der Wellenzahl der Periodizität entlang der $x_{c}$-Richtung und der negative Imaginärteil von $\alpha^{\prime}$ beschreibt die räumliche Anfachungsrate der Störung in dieser Richtung. Im Folgenden wird für die Wellenzahl in $x_{c}$-Richtung das Symbol $\alpha=\Re\left(\alpha^{\prime}\right)$ und für die Anfachungsrate das Symbol $\sigma=-\Im\left(\alpha^{\prime}\right)$ eingeführt. Die $x_{c}$-Position, an der die Anfachungsrate einer Instabilitätsmode ihr Vorzeichen von negativ zu positiv wechselt, also ab der diese Mode in der Grenzschicht angefacht ist, nennt man Neutralpunkt dieser Mode. Im Rahmen der Arbeit wurden mithilfe der für kompressible und inkompressible Strömungen anwendbaren Software NOLOT [45] lineare, lokale und parallele Stabilitätsuntersuchungen durchgeführt (siehe Abschnitte 3.2 und 6.2), bei denen der Normalmodenansatz aus Gleichung (2.2.7) verwendet wurde, um 
die Stördifferentialgleichungen 2.2.5 und 2.2.6 für die Randbedingungen des betrachteten Experiments zu lösen.

\subsection{Der querströmungsdominierte inkompressible Transitionsprozess}

Im Folgenden sollen die bisherigen wissenschaftlichen Erkenntnisse dargestellt werden, die für die Diskussion der Ergebnisse dieser Arbeit erforderlich sind. Für einen detaillierteren Überblick über den querströmungsdominierten Transitionsprozess siehe $[8,87,97]$.

Unter unendlich schiebenden Bedingungen erfährt die Strömung unter Einfluss des Druckgradienten entlang $x_{c}$ eine Beschleunigung in dieser Richtung, während die spannweitige Geschwindigkeitskomponente $v_{c}$ unbeeinflusst ist. Dies bewirkt eine Krümmung der Grenzschichtrandstromlinie. Diese Stromlinie verläuft nahe der Anlegelinie überwiegend spannweitig und ändert aufgrund der Beschleunigung durch den Druckgradienten und des in $x_{c^{-}}$ Richtung wachsenden Betrags der $u_{c}$-Komponente ihre Richtung. Am oberen Grenzschichtrand besteht ein Gleichgewicht zwischen der Zentrifugalkraft der beteiligten Fluidteilchen und der entgegengerichteten Komponente des Kraftbetrags des Druckgradienten. Innerhalb der Grenzschicht ist durch den Reibungseinfluss der Betrag der Geschwindigkeit der beteiligten Fluidteilchen und damit ihre Zentrifugalkraft gegenüber dem Wert am Grenzschichtrand herabgesetzt. Der statische Druck ändert sich aber in wandnormaler Richtung innerhalb der Grenzschicht kaum, sodass die resultierende Strömungsrichtung der Fluidteilchen in der Grenzschicht von derjenigen am Grenzschichtrand abweicht. Der Geschwindigkeitsvektor der Fluidteilchen in der Grenzschicht ist relativ zur Strömungsrichtung am oberen Grenzschichtrand in Richtung des Krümmungsmittelpunkts der gekrümmten Potentialstromlinie geneigt. Begibt man sich in das Koordinatensystem, welches sich an der gekrümmten Potentialstromlinie (am Grenzschichtrand) orientiert, existiert innerhalb der Grenzschicht zusätzlich zu der Komponente $u_{s}$ in Hauptströmungsrichtung eine Komponente $v_{s}$ in Richtung des Krümmungsmittelpunkts, wie schematisch in Abb. 2.3.1 skizziert ist. Dabei erkennt man die vom Wandabstand abhängige Strömungsrichtung im schwarz dargestellten resultierenden Grenzschichtprofil, illustriert durch schwarze Pfeile.

Der wandnormale Verlauf dieser Geschwindigkeitskomponente $v_{s}$ weist einen Wendepunkt auf, welcher Quelle einer reibungsfreien Instabilität ist. Sie wird 


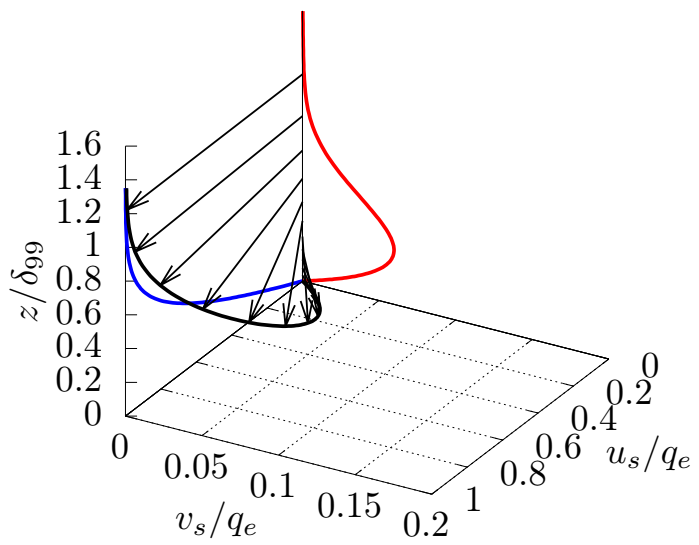

Abbildung 2.3.1: Schematische Darstellung der Geschwindigkeitsprofile im stromlinienorientierten Koordinatensystem (Die Pfeile stellen die Strömungsrichtung an ausgewählten Wandabständen dar)

in diesem Fall Querströmungsinstabilität genannt und wurde zuerst von Gray [36] entdeckt und später durch Gregory et al. [37] theoretisch behandelt. Die Wendepunktinstabilität führt dazu, dass gewisse Störungen der Strömung nicht gedämpft, sondern angefacht werden. Die angefachten Störungen in der Grenzschicht, welche in der linearen Stabilitätstheorie als Instabilitätsmoden gemäß eines Wellenansatzes beschrieben werden (siehe Abschnitt 2.2), werden verkürzt auch oft als Querströmungsinstabilitäten (QSI) bezeichnet. Sie entsprechen phänomenologisch gleichsinnig rotierenden Längswirbeln in der Grenzschicht, deren Wirbelachsen mit einer leichten Winkeldifferenz nahezu der Grenzschichtrandstromlinie folgen. Diese Längswirbel heben impulsarmes Fluid nahe der Wand nach oben und transportieren impulsreiches Fluid von oben zur Wand hin. Dieser Impulstransport bewirkt eine deutlich messbare lokale Deformation des stationären Geschwindigkeitsfeldes.

Angefachte Querströmungsinstabilitäten treten sowohl als laufende sowie als stationäre Moden auf. Stationäre QSI besitzen eine Kreisfrequenz $\omega=0$. Ihre Deformation des Grenzschichtströmungsfeldes ist zeitunabhängig und ortsfest relativ zur Modelloberfläche, weshalb sie sich zur rasterförmigen Untersuchung mit Hitzdrahtsonden besonders eignen. Laufende QSI besitzen eine nicht-verschwindende Kreisfrequenz $\omega$. Ihre Deformation des Grenzschichtströmungsfeldes ist zeitabhängig und nicht ortsfest relativ zur Modelloberfläche, sodass sie in rasterförmigen Hitzdrahtmessungen zunächst nur durch Geschwindigkeitsfluktuationen mit Frequenz $f=\omega / 2 \pi$ in Erscheinung tritt. 


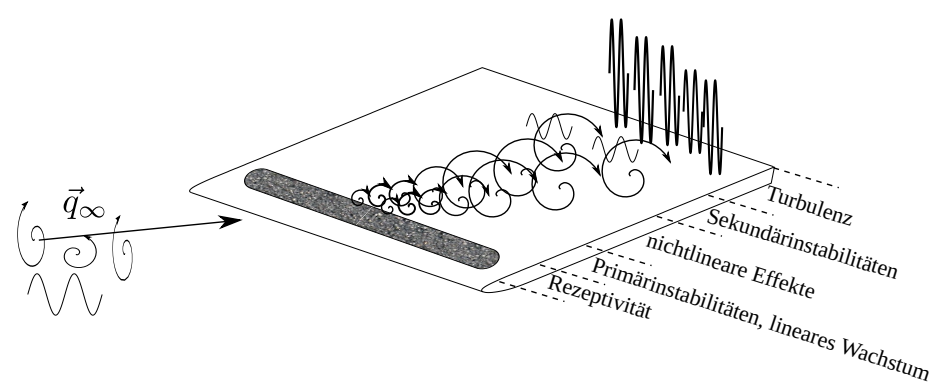

Abbildung 2.3.2: Schematische Darstellung des querströmungsdominierten Transitionsprozesses auf der Modellplatte mit stationären Querströmungsinstabilitäten als Beispiel

In Experimenten mit kontrollierter instationärer Anregung laufender QSI lässt sich durch das Festlegen eines Phasenbezuges zwischen Anregung und Messung auch die räumliche Struktur der Grenzschichtdeformation durch laufende QSI mit rasterförmigen Hitzdrahtmessungen untersuchen (z.B. in [62]). Da die Anregung in den Experimenten im Rahmen dieser Arbeit nicht kontrolliert wurde, ist dies hier nicht möglich.

Neben der Querströmungsinstabilität treten in dreidimensionalen Grenzschichten noch andere Instabilitätstypen auf: Anlegelinieninstabilität, Instabilität in Strömungsrichtung und Zentrifugalinstabilität [97]. Anlegelinieninstabilität tritt üblicherweise an schiebenden Flügeln mit großen Nasenkrümmungsradien entlang der Anlegelinie auf [82, 87]. Die Instabilität in Strömungsrichtung ist der Instabilität in zweidimensionalen Grenzschichten ähnlich, in denen sich Tollmien-Schlichting-Instabilitäten entwickeln [99, 108]. Ein negativer Druckgradient in Hauptströmungsrichtung wirkt stabilisierend auf diese Instabilitäten, während ein positiver Druckgradient destabilisierend wirkt. Zentrifugalinstabilität tritt an Positionen in der Grenzschicht über einer konkaven Oberflächenkrümmung in Form von Görtler-Wirbeln auf [40]. Das im Rahmen dieser Arbeit untersuchte Experiment wurde unter anderem mit dem Ziel ausgelegt, möglichst isoliert Querströmungsinstabilität zu untersuchen (siehe Abschnitt 4.2), weshalb im Folgenden nur noch dieser Instabilitätstyp diskutiert wird.

Nach heutigem Forschungsstand lässt sich der querströmungsdominierte Transitionsprozess in aufeinanderfolgende Stadien entlang der Hauptströmungsrichtung aufteilen. Diese Stadien sind in Abbildung 2.3.2 schematisch dargestellt und werden im Detail in den folgenden Abschnitten einzeln beschrieben. 
Im Prozess der Rezeptivität koppeln initiale Störungen in die Grenzschicht ein. Beispiele für solche Störungen sind Turbulenz in der Anströmung oder Auslenkungen des Fluids durch Unregelmäßigkeiten der Modelloberfläche bzw. Rauigkeit. Aus dieser initialen Anregung entstehen Instabilitätsmoden, z.B. QSI. Diese Moden werden auch als Primärinstabilitäten bezeichnet. Man unterscheidet zwischen primärer und sekundärer Instabilität, da bei ausreichend hohen Amplituden der Primärinstabilitäten ihre Deformation der Grenzschichtströmung zu einer zusätzlichen Instabilität der Grenzschicht führen kann, der Sekundärinstabilität. Solange die Amplituden der Primärinstabilitäten ausreichend klein für die Vernachlässigung nichtlinearer Terme in den Stördifferentialgleichungen sind, wird ihr Amplitudenwachstum durch die lineare Theorie korrekt beschrieben und man spricht von linearem Wachstum. Sobald dies nicht mehr der Fall ist, treten nichtlineare Effekte auf. Die Deformation der Grenzschicht durch die Primärinstabilitäten ändert lokal ihre Stabilität und Sekundärinstabilitäten treten auf, wonach meist kurz stromab der laminar-turbulente Umschlag der Grenzschicht erfolgt.

\section{Rezeptivität}

Ausgangspunkt für Instabilitätsmoden in der laminaren Grenzschicht gemäß Gleichung 2.2.7 sind kleine Anfangsstörungen. Dabei sind Störungen aus der Anströmung oder Eigenschaften des umströmten Körpers als Ursache denkbar. Der Prozess des Entstehens von Grenzschichtinstabilitäten aus Anfangsstörungen wird seit den Arbeiten von Morkovin [74] Rezeptivitätsprozess genannt, da die instabile Grenzschicht im Rahmen dieses Prozesses empfänglich (rezeptiv) für initiale Störungen ist. Ein detaillierter Überblick über die Erkenntnisse zu diesem Prozess wird bei Saric [96] gegeben.

Die relevante Anregung für stationäre Querströmungsinstabilitäten konnte durch B. Müller [76] eindeutig identifiziert werden. Nach der Vermessung der stationären Grenzschichtdeformation durch stationäre QSI mithilfe von Hitzdrahtmessungen verschob er das Modell in spannweitiger Richtung und fand bei erneuten Hitzdrahtmessungen die stationäre Grenzschichtdeformation mit gleicher räumlicher Struktur an denselben Positionen im modellfesten Koordinatensystem wie zuvor. Zusätzlich untersuchten Deyhle und Bippes [22] die spannweitige Variation der Anströmung und fanden keine Korrelation zur spannweitigen Periodizität der stationären QSI in der Grenzschicht. Die relevante Anregung von stationären Querströmungsinstabilitäten muss also der Modelloberfläche und nicht der Anströmung entstammt sein. 
Zur Anregung stationärer Querströmungsinstabilitäten haben Radeztsky et al. [84] den Einfluss von isolierten Einzelrauigkeiten (im Folgenden wird die gebräuchliche englische Abkürzung DRE für "discrete roughness element" verwendet) und den Einfluss von verteilter bzw. „natürlicher" Rauigkeit untersucht. Dabei stellten sie fest, dass die Sensitivität des Transitionsprozesses bzgl. Anregung stationärer QSI durch ein DRE am größten nahe oder kurz stromauf des Neutralpunkts der instabilsten stationären Moden ist, weshalb die Rauigkeit in Abbildung 2.3.2 nur im vorderen Bereich der Modellplatte angedeutet ist. Ein weiteres Ergebnis der Untersuchungen war, dass die Transitionslage stark von der Rauigkeitshöhe eines DRE abhängt. Des Weiteren war die Anregung stationärer QSI nur dann effektiv, wenn der Durchmesser des DRE mindestens 10\% der spannweitigen Wellenlänge der meistangefachten stationären QSI entsprach. Zum Einfluss verteilter Rauigkeit erhielten Radeztsky et al. das Ergebnis, dass die Transitionslage auch stark von der mittleren Amplitude verteilter Rauigkeit abhängt. Die durch die Rauigkeit induzierte Anfangsstörung in der Grenzschicht ist aber von so kleinem Betrag, dass sie sich einer direkten Messung bislang entzieht. Im Rahmen dieser Arbeit werden Unregelmäßigkeiten in der Modelloberfläche allgemein als Rauigkeit bezeichnet. In dieser Wortwahl soll keine Einschränkung des Wellenzahlbereichs enthalten sein, auch wenn in der Alltagssprache eher kurzwellige Unregelmäßigkeiten gemeint sind.

Für die Anregung laufender QSI sind instationäre Anfangsstörungen aus der Anströmung wichtig. Müller [76] und Deyhle [21] untersuchten das Transitionsszenario an der Oberfläche desselben Modells in unterschiedlichen Windkanälen mit unterschiedlichem Turbulenzgrad der Anströmung. Dabei bestimmten sie jeweils die Amplituden stationärer und laufender QSI und fanden heraus, dass bei relativ geringem Turbulenzgrad stationäre QSI das Transitionsszenario dominieren, während bei relativ hohem Turbulenzgrad laufende QSI höhere Amplituden erreichen und das Transitionsszenario bestimmen. Als Grenzbereich für die Dominanz stationärer QSI nennen Deyhle und Bippes einen Wertebereich des Turbulenzgrads der Anströmung zwischen $0.15 \%<T u<0.2 \%$ [22]. Bei höheren Turbulenzgraden erwarten sie die Dominanz laufender Moden, bei niedrigeren Werten die Dominanz stationärer Moden. Letzteres ist wegen des niedrigen Turbulenzgrads insbesondere im Freiflug gegeben [97], was ein besonderes wissenschaftliches Interesse für solche Transitionsszenarien motiviert.

Bei der Betrachtung der Störungen in der Anströmung, welche die Anströmturbulenz ausmachen, muss man zwischen akustischen und wirbelartigen 
Störungen unterscheiden [8]. Radeztsky et al. [84] untersuchten den Einfluss einer Schallquelle mit hohen Schalldrücken in der Anströmung bei allen Frequenzen angefachter laufender Instabilitäten. Dabei stellte sich heraus, dass die Schallquelle keinen beobachtbaren Effekt auf das Transitionsszenario am untersuchten Modell hatte. Einen ähnlichen Versuch unternahmen Deyhle und Bippes [22] und verglichen Spektren instationärer Geschwindigkeitsschwankungen aus Hitzdrahtmessungen außerhalb und in der Grenzschicht des untersuchten Modells. Dabei fanden sie eine Anhebung der Amplituden im angeregten Frequenzbereich nur außerhalb der Grenzschicht, in der Grenzschicht waren die Spektren unverändert. Die entscheidende Quelle für laufende QSI muss im Unterschall also der wirbelartige Anteil der Anströmturbulenz sein [8].

Borodulin et al. [14] erzeugten gezielt in der Anströmung vor ihrem Modell eine solche wirbelartige Anregung durch einen spannweitig gespannten vibrierenden Draht, sodass die erzeugte Geschwindigkeitsschwankung keine spannweitige Periodizität aufwies. Auf der Modelloberfläche platzierten sie eine wohldefinierte Rauigkeit, deren Wellenzahlspektrum in $x_{c}$-Richtung und $y_{c}$-Richtung so gestaltet war, dass das Spektrum der Oberflächenstruktur möglichst stark dem der angefachtesten Mode laufender QSI entsprach. Stromab von der Rauigkeit konnten laufende QSI mit entsprechender Frequenz und den entsprechenden räumlichen Wellenzahlen nachgewiesen werden. Sie konnten auch zeigen, dass diese QSI nur durch Streuung an der künstlichen Rauigkeit entstanden sind. Für den Rezeptivitätsprozess laufender QSI im Allgemeinen sind also sowohl Geschwindigkeitsschwankungen in der Anströmung als auch die räumliche Periodizität der Modelloberflächenstruktur relevant.

\section{Lineares Wachstum}

Die im Rezeptivitätsprozess entstandenen Instabilitätsmoden werden gemäß der Stabilitätseigenschaften in der Grenzschicht angefacht. Im Allgemeinen sind laufende Querströmungsinstabilitäten immer stärker angefacht als stationäre. Mit dem Bereich linearen Wachstums ist dabei der Profiltiefenbereich gemeint, in dem das Amplitudenwachstum der Instabilitätsmoden durch die mit der linearen Stabilitätstheorie bestimmten Anfachungsraten korrekt beschrieben wird. Die Amplitudenentwicklung einer Instabilitätsamplitude $A$ folgt dann der entlang $x_{c}$ aufintegrierten Anfachungsrate $\sigma$, dem sog. N-Faktor, $N=\int \sigma d x_{c}$, gemäß $A=A_{0} e^{N}$ mit einer Anfangsamplitude $A_{0}$ als Ergebnis des Rezeptivitätsprozesses. Da in der linearen Stabilitätstheorie nichtlineare Terme vernachlässigt wurden, gilt diese theoretische Behandlung nur solange, 
bis die Instabilitäten einen nicht mehr als klein anzunehmenden Amplitudenbetrag erreichen. Die am Transitionsprozess beteiligten Primärinstabilitäten und ihre Amplituden bestimmen aber maßgeblich die nichtlinearen Effekte stromab. Die lineare Theorie beschreibt korrekt die Wellenzahlen der am stärksten angefachten stationären QSI [4, 22, 49, 50, 76, 79] und Frequenzen und Wellenzahlen der am stärksten angefachten laufenden QSI voraus $[8,22$, 50]. Die unterschiedlich starke Anfachung von Instabilitätsmoden abhängig von ihren Wellenzahlen und Frequenzen stellt eine Auswahl aus der initialen Anregung dar und man spricht von einer selektiven Anfachung.

Die QSI deformieren im linearen Bereich idealisiert betrachtet nur lokal das Grenzschichtprofil, das spannweitig gemittelte Profil bleibt jedoch unbeeinflusst. Die Deformation durch stationäre QSI tritt in rasterförmigen Hitzdrahtmessungen also durch lokale ortsfeste Geschwindigkeitsdifferenzen zum spannweitigen Mittelwert der zeitlich gemittelten Geschwindigkeit in Erscheinung, während laufende QSI an jeder spannweitigen Position der Rastermessung als zeitliche Schwankung der Geschwindigkeit mit spannweitig gleicher Amplitude auftreten. Für beide Instabilitätsformen ist der Betrag der Amplitude gemäß der Amplitudenfunktion der jeweiligen Mode abhängig vom Wandabstand.

\section{Nichtlineare Effekte}

Im ursprünglichen Prinzipexperiment der schiebenden ebenen Platte wurde von Müller, Deyhle und Lerche [21, 62, 76] das Phänomen beobachtet, dass die Amplituden stationärer QSI weit stromauf vom finalen Umschlag einen Plateauwert, auch Sättigungsamplitude genannt, erreichten, der bis zum finalen Umschlag nahezu unverändert blieb. Da die betrachteten Moden nach linearer Theorie ab dem Neutralpunkt über die gesamte Plattentiefe angefacht waren, entsprach dies nicht der nach linearer Theorie erwarteten Amplitudenentwicklung. Reibert et al. [90] führten ebenfalls Experimente zur Sättigung der Amplitude stationärer QSI durch. Sie variierten die relevante Anregungsamplitude für stationäre QSI mithilfe der Rauigkeitshöhe einer spannweitig periodischen Anordnung von DRE. Es zeigte sich, dass der Wert der Sättigungsamplitude der stationären QSI nahezu unabhängig von der Höhe der anregenden Reihe aus DRE war, obwohl der Einfluss der Rauigkeitshöhe im linearen Bereich sichtbar war [89, 94]. Bei einer stärkeren künstlichen Anregungsamplitude wird das Sättigungsniveau dafür früher erreicht [8]. 
Müller [76] und Deyhle [21] stellten bei ihren Untersuchungen in unterschiedlichen Windkanälen mit unterschiedlicher Anströmturbulenz auch unterschiedliche Sättigungsamplituden der stationären QSI fest. Bei hohem Wert des Turbulenzgrades in der Anströmung erreichten die stationären QSI eine niedrigere Sättigungsamplitude als bei niedriger Anströmturbulenz. Daraufhin entwickelte Lerche [62] einen Aktuator, mit dem es möglich war, eine diskrete Mode laufender QSI mit definierter Frequenz und spannweitiger Wellenzahl $\beta$ künstlich anzuregen. Eine diskrete Mode stationärer QSI konnte optional mithilfe spannweitig periodischer DRE angeregt werden. Er fand heraus, dass in einem Fall, in dem sowohl eine stark angefachte Mode laufender QSI und eine stark angefachte Mode stationärer QSI angeregt wurde, die Amplitude der stationären QSI verringert war gegenüber dem Fall, in dem nur die stationäre Mode allein angeregt wurde. Durch die parallele Anwesenheit der laufenden QSI war durch eine nichtlineare Interaktion das Wachstum der stationären QSI gedämpft. Müller [76] stellte auch fest, dass bei der Betrachtung der Geschwindigkeitsfluktuationen aufgrund laufender QSI spannweitig lokalisiert im Strömungsfeld stark unterschiedliche Amplituden auftraten. Diese spannweitige Variation der Amplituden instationärer Geschwindigkeitsfluktuationen besaß dieselbe spannweitige Wellenlänge wie die der stationären QSI, während nach linearer Theorie eine spannweitig konstante Amplitude erwartet wird. Dies wies auf eine nichtlineare Wechselwirkung zwischen stationären und laufenden QSI hin.

In seinen experimentellen Untersuchungen wies Reibert [90] nach, dass nichtlinear auch neue Instabilitätsmoden mit ganzzahligen Vielfachen der linear angefachten Wellenzahl der Primärinstabilität erzeugt werden können. Im Gegensatz zu solchen höherharmonischen Instabilitäten wurden keine nichtlinear erzeugten Subharmonischen (mit geringerer Wellenzahl) beobachtet. Lerche [62] fand durch die gezielte Anregung einzelner Moden stationärer und laufender QSI heraus, dass die nichtlineare Interaktion dieser Moden auch Moden mit Vielfachen der Frequenz sowie mit Vielfachen der spannweitigen Wellenzahl der linear angefachten Primärinstabilitäten erzeugen kann.

Basierend auf den Untersuchungen von Reibert untersuchten Saric et al. [95] den Effekt der künstlichen Anregung einer stationären Instabilitätsmode mit einer größeren spannweitigen Wellenzahl $\beta$ als die der linear instabilsten Mode. Sie beobachteten eine Transitionsverzögerung als Folge einer verringerten Amplitude der instabilsten stationären QSI-Mode gegenüber dem Fall ohne künstliche Anregung. Mithilfe von Direkter Numerischer Simulation untersuchten Wassermann \& Kloker [112] einen solchen Fall einer künstlichen 
Anregung und konnten ebenfalls eine Transitionsverzögerung nachweisen. Sie gaben dieser neuen Methode zur Transitionsverzögerung den Namen Upstream Flow Deformation (UFD), welcher sich inzwischen in der Literatur durchgesetzt hat. Aufgrund seiner zentralen Bedeutung für diese Arbeit ist dieser Methode ein eigener Abschnitt gewidmet, siehe Abschnitt 2.4.

In den Messungen von Lerche [62] wurde auch deutlich, dass an Positionen, an denen die stationären QSI ihre Sättigungsamplitude erreicht hatten, zusätzlich zu der lokalen Deformation der Grenzschichtprofile eine Deformation der spannweitig gemittelten Profile im Vergleich zum Fall ohne künstlicher Anregung stattfand. Diese Deformation der Grundströmung ist durch eine nichtlineare Wechselwirkung zwischen der stationären Grundströmung und den QSI begründet und wird als Mean Flow Distortion (MFD) bezeichnet [8]. Die Konsequenz einer erzeugten MFD bedeutet, dass mit der Form des spannweitig gemittelten Geschwindigkeitsprofils auch die Stabilitätseigenschaften der Grenzschicht signifikant gegenüber der ungestörten Grundströmung verändert sind.

\section{Sekundärinstabilitäten}

Wie oben beschrieben deformieren QSI lokal die Geschwindigkeitsprofile der Grenzschicht. Bei einer ausreichend starken lokalen Deformation der Profile können diese zusätzlich instabil werden, auch bzgl. Störungen, welche primär nicht angefacht sind. Solche Instabilitäten folgen im Transitionsprozess stromab auf die Primärinstabilitäten und werden deshalb Sekundärinstabilitäten genannt.

Arnal et al. [4] und Poll [83] beobachteten als Erste kurz stromauf des turbulenten Umschlags das Auftreten hochfrequenter instationärer Störungen, deren Frequenz eine Größenordnung über der Frequenz laufender QSI lag. Diese Beobachtung konnte durch Experimente von Kohama [55], Bippes [10] und Deyhle \& Bippes [22] bestätigt werden. Kohama et al. [55] zeigten, dass diese hochfrequenten Störungen im Strömungsfeld spannweitig stark lokalisiert an Positionen starker Grenzschichtdeformation durch stationäre QSI auftraten und interpretierten sie als Sekundärinstabilitäten. White \& Saric [113] machten die Beobachtung, dass solche Sekundärinstabilitäten viel stärker angefacht sind als Primärinstabilitäten und fanden die Transition immer innerhalb von 5\% der Profillänge ab dem Auftreten der Sekundärinstabilitäten. Es wird ein kausaler Zusammenhang zwischen ihnen und dem turbulenten Umschlag vermutet [97]. 
Aufgrund der künstlichen Anregung einzelner laufender Instabilitätsmoden konnte Lerche $[9,61,62]$ phasenstarre Messungen der durch die angeregten laufenden QSI deformierten Grenzschichtprofile durchführen. Dabei stellte sich heraus, dass die hochfrequente Sekundärinstabilität immer dann auftrat, wenn das instantan deformierte $u_{s}$-Profil einen Wendepunkt aufwies. Sekundärinstabilitäten können also nicht nur durch eine stationäre lokale Deformation der Grenzschichtprofile auftreten, sondern auch zu ausgewählten Zeitpunkten durch eine instationäre Grenzschichtdeformation.

In numerischen Untersuchungen konnten zugrundeliegende Mechanismen der Sekundärinstabilitäten identifiziert und drei unterscheidbare Klassifizierungen eingeführt werden [54, 71, 72]. Demnach wird unterschieden zwischen Sekundärinstabilitäten vom Typ I, welche örtlich mit hohen Werten spannweitiger Scherung der Hauptströmungskomponente in Wirbelachsenrichtung korrelieren [71], Sekundärinstabilitäten vom Typ II, welche örtlich mit hohen Werten wandnormaler Scherung dieser Komponente korrelieren [71] und Sekundärinstabilitäten vom Typ III, welche ebenfalls örtlich mit hohen Werten wandnormaler Scherung dieser Komponente korrelieren [54]. Der Typ III wird allerdings wandnah erwartet [54], im Gegensatz zu Typ II, welcher in größerem Wandabstand, nahe des Grenzschichtrands erwartet wird [71]. Da diese Scherspannungen durch die Grenzschichtdeformation der Primärinstabilitäten entstehen, werden Sekundärinstabilitäten erst bei hohen Amplituden der primären QSI beobachtet. Sekundärinstabilitäten können auch im selben Frequenzbereich auftreten wie laufende Primärinstabilitäten. Treten stationäre QSI mit hoher Amplitude auf, treten instationäre Störungen im Frequenzbereich laufender QSI als niederfrequente Sekundärinstabilitäten auf [44].

Wassermann \& Kloker [112] konnten in DNS-Untersuchungen (Direkte Numerische Simulation) nachweisen, dass Sekundärinstabilitäten konvektive und keine absoluten Instabilitäten sind. Da Sekundärinstabilitäten von dem durch Primärinstabilitäten deformierten Grenzschichtprofil verursacht werden, treten sie in Transitionsszenarien mit dominanten stationären QSI stark lokalisiert auf. Eine spannweitig inhomogene Transitionsfront wird beobachtet, während in einem von laufenden QSI dominierten Szenario die Sekundärinstabilitäten und die Transitionsfront spannweitig homogen verteilt beobachtet werden [97]. 


\subsection{Verzögerung der querströmungsdominierten Transition}

Ein Ansatz zur Verzögerung der Transition ist die Stabilisierung der Grenzschicht gegenüber den Störungen, welche für die Transition verantwortlich sind. Dies ist vor allem in der Auslegungsphase eines schiebenden Flügels durch eine geeignete Formgebung möglich. Alternativ kann die Amplitude der Störungen durch einen aktiven Eingriff reduziert werden. Wie im vorhergehenden Abschnitt 2.3 beschrieben, wurde der finale Umschlag im querströmungsdominierten Fall stets kurz nach dem Auftreten von Sekundärinstabilitäten beobachtet, sodass ein kausaler Zusammenhang vermutet wird. Gelingt es, ihr Auftreten zu verzögern, erwartet man, dass auch die Transition verzögert wird. Auf dem Entwicklungspfad bis zu den Sekundärinstabilitäten gibt es mehrere denkbare Eingriffsoptionen. Da die Sekundärinstabilitäten an Orten in der Grenzschicht auftreten, an denen die Primärinstabilitäten eine große Deformation eingebracht haben, liegt es nahe, die Primärinstabilitäten in ihrer Amplitude zu reduzieren. In der Phase der Rezeptivität kann man versuchen, die initiale Anregung der stationären und laufenden QSI zu begrenzen. Da man im Allgemeinen keinen Einfluss auf die Anströmturbulenz hat, bietet sich vor allem an, für eine Oberflächenbeschaffenheit zu sorgen, welche im relevanten Wellenlängenbereich möglichst geringe Anteile enthält. In der Phase des linearen Instabilitätswachstums kann man das spannweitig gemittelte Grenzschichtprofil durch betragliche Reduktion der Querkomponente $v_{s}$ bgzl. QSI stabilisieren. Als aktive Maßnahmen dafür wurden z.B. eine verteilte Absaugung [1, 2, 8] und Plasmaaktuatoren [23, 24, 58] untersucht. Weiterhin wurde rein numerisch die Möglichkeit untersucht, direkt primäre QSI durch lokale Absaugung oder durch Plasmaaktuatoren bzgl. ihrer sekundären Instabilität zu stabilisieren [25, 33]. Dies ist experimentell schwer umzusetzen, da es die genaue Kenntnis der Phasenlage der Primärinstabilitäten an der jeweiligen Aktuierungsposition erfordert.

\subsubsection{Upstream Flow Deformation}

Ein besseres Verständnis der nichtlinearen Effekte ermöglichte eine neue Methode zur Transitionsverzögerung, die Upstream Flow Deformation, siehe bereits Abschnitt 2.3. Eines der Ergebnisse der Experimente von Reibert et al. [90] zum nichtlinearen Verhalten stationärer QSI war, dass bzgl. der spannweitigen Wellenzahl höherharmonische Störungen, aber keine subhar- 
monischen Störungen nichtlinear erzeugt werden. Daraufhin untersuchten Saric et al. [95] den Einfluss einer zusätzlich künstlich angeregten stationären QSI-Mode, deren spannweitige Wellenzahl $\beta$ größer gewählt wurde als die derjenigen stationären QSI-Mode, welche natürlicherweise den Umschlag dominierte. Dies wurde wegen der zur Wellenzahl reziproken Wellenlänge $\lambda$ als ,subkritische Anregung“" und die künstlich angeregte Mode als „,control mode" bezeichnet. In Anlehnung an die englische Bezeichnung wird die künstlich anzuregende Mode auch in dieser Arbeit als „Kontrollmode" bezeichnet. Saric et al. wählten als spannweitige Wellenlänge dieser künstlich angeregten Mode zwei Drittel der spannweitigen Wellenlänge der natürlich dominanten Mode. Die zu wählende Wellenlänge hängt neben anderen Eigenschaften der Grundströmung insbesondere von der Reynoldszahl der Anströmung ab. Bei ihren Untersuchungen beobachteten Saric et al. eine Dämpfung der natürlich dominanten QSI und eine signifikante Transitionsverzögerung.

Wassermann \& Kloker [112] konnten eine solche Situation mithilfe von DNS nachbilden und zeigen, dass die Dämpfung maßgeblich auf die zweidimensionale Verformung der spannweitig und zeitlich gemittelten Geschwindigkeitsprofile zurückzuführen ist. Diese MFD wird nichtlinear von der künstlich angeregten Instabilitätsmode generiert und hat eine stabilisierende Wirkung. Daraus ergibt sich die Anforderung an die Grundströmung, dass in ihr die Kontrollmode ausreichend stark angefacht ist und im Transitionsprozess ausreichend stromauf zu hohen Amplituden führt. Dadurch kann die MFD ebenfalls ausreichend stromauf mit signifikanter Amplitude nichtlinear erzeugt werden. Dies muss geschehen, bevor die natürlich dominante Mode sekundär instabil wird. Die stabilisierende Wirkung der Verformung des spannweitig gemittelten Profils ist auch natürlicherweise für die Sättigung der QSI-Amplituden verantwortlich. Diese Wirkung wird durch die UFDMethode also nur stromauf verlagert. Man spricht vereinfachend auch von einer Vordeformation der Grenzschicht.

Der klare Vorteil dieser Methode zur Transitionsverzögerung im Gegensatz zu den im vorigen Absatz genannten linearen Beeinflussungsansätzen ist, dass nur eine sehr geringe Aktuierungsleistung benötigt wird, weil gezielt eine Instabilität der Grenzschicht und die Anfachung der initialen Anregung durch die Grenzschicht benutzt wird, um einen nichtlinearen Effekt zu provozieren. Die Methode hat dadurch das Potential, auf sehr energieeffiziente Weise eine Transitionsverzögerung zu erreichen. Die nichtlineare Funktionsweise macht die Methode aber sehr empfindlich gegenüber den Anfangsbedingungen. Es ist daher leicht ersichtlich, dass die geeignete Wahl von Wellenlänge und 
Amplitude der künstlich angeregten stationären Kontrollmode von großer Bedeutung ist und insbesondere empfindlich von den Anströmungsbedingungen abhängt, was den Nachteil der Methode darstellt. Die geeignete Wahl von Wellenlänge und Amplitude der Kontrollmode hängt dabei nicht nur von den Eigenschaften der Grenzschichtgrundströmung, sondern auch von den Eigenschaften der Störströmung im unbeeinflussten Fall ab. Tatsächlich sind in der Literatur viele Experimente bekannt, in denen der Einsatz der UFDMethode nicht wie beabsichtigt zu einer Transitionsverzögerung geführt hat (siehe bspw. [98, 105]). Nicht immer konnte dabei die Ursache geklärt werden.

Zur künstlichen Anregung der stationären Kontrollmode verwendeten Saric et al. [95] spannweitig periodisch auf der Modelloberfläche aufgetragene diskrete zylindrische Rauigkeitselemente (DRE). Die Anpassung der Wellenlänge und Amplitude dieser künstlichen Anregung lässt sich nur durch Austausch der DRE realisieren. Nachdem mithilfe der UFD-Methode unter Einsatz von DRE in mehreren Fällen eine Transitionsverzögerung nachgewiesen werden konnte [17, 95], wurden Methoden untersucht, um zumindest die Amplitude der Kontrollmode flexibel variieren zu können. Schuele et al. [101] benutzten einen spannweitig periodisch angeordneten Plasmaaktuator, um stationäre QSI im Sinne der UFD-Methode anzuregen. Leider trafen Sie keine Aussage darüber, ob eine Transitionsverzögerung erreicht wurde oder nicht. Lohse et al. [68] verwendeten einen pneumatischen Aktuator mit spannweitig periodisch angeordneten Bohrungen, durch die alternativ mittels zeitlich konstanter Absaugung oder Ausblasen stationäre QSI angeregt wurden, um die UFDMethode anzuwenden. Die Experimente fanden in derselben experimentellen Konfiguration wie die Experimente dieser Arbeit statt und es konnte für bestimmte Anregungsparameter eine signifikante Dämpfung der natürlich dominanten stationären QSI und eine signifikante Transitionsverzögerung nachgewiesen werden.

Während in numerischen Simulationen der erfolgreiche Einsatz von modellierten Plasmaaktuatoren zur Transitionsverzögerung mithilfe der UFD-Methode gezeigt werden konnte [26, 106], konnte eine Verzögerung des finalen Umschlags mittels Plasmaaktuatoren durch UFD bislang nicht in Experimenten nachgewiesen werden $[53,104]$. Dabei ist zu beachten, dass die instationäre Funktionsweise eines Plasmaaktuators die Gefahr birgt, unabsichtlich zusätzlich zu stationären QSI auch laufende QSI anzuregen, welche selbst zu einer stromauf verlagerten Transition führen können [104]. 


\subsection{Gezielte Anregung stationärer Querströmungsinstabilitäten}

Die künstliche Anregung stationärer QSI ist in der Forschung für die Überprüfung numerischer Methoden oder theoretischer Modelle im Rahmen des Transitionsprozesses in Grenzschichten an schiebenden Flügeln interessant. Durch kontrollierte Variation der Anfangsamplitude kann überprüft werden, ob die Entwicklung der Mode stromab numerisch oder theoretisch korrekt beschrieben wird. Durch die kontrollierte parallele Anregung mehrerer definierter Moden konnte das Verständnis nichtlinearer Wechselwirkungen maßgeblich erweitert werden [62].

Spätestens aber seit den Arbeiten von Saric [95] und der Idee, die nichtlineare Wechselwirkung von Instabilitätsmoden zur Transitionsverzögerung zu benutzen (siehe Abschnitt 2.4), wurden unterschiedliche Methoden zur künstlichen Anregung von stationären QSI untersucht. Nachdem Müller \& Bippes [76, 77] die Oberflächenrauigkeit als entscheidenden Auslöser des Rezeptivitätsmechanismus stationärer QSI identifiziert hatten (siehe Abschnitt 2.3), untersuchten Radeztsky et al. [84] den Einfluss von Rauigkeitshöhe und -durchmesser auf die stationären Instabilitäten in der Grenzschicht. Zu den im Rahmen dieser Arbeit untersuchten Anregungsmethoden sollen in den folgenden Abschnitten die vorhandenen Erkenntnisse und Hypothesen vorgestellt werden. Da die Mechanismen der Anregung einer stationären QSI meist räumlich sehr kleinskalig und die beteiligten Störungsgrößen initial von sehr kleinem Betrag sind, entziehen sie sich experimenteller Untersuchung. Daher basieren viele Erkenntnisse rein auf numerischen Untersuchungen.

\subsubsection{Diskrete Einzelrauigkeiten}

Der Einfluss zylindrischer diskreter Rauigkeitselemente wurde schon früh in zweidimensionalen Grenzschichten, also ohne Querströmung untersucht. Gregory \& Walker [38] fanden einen stationären Hufeisenwirbel an der Einzelrauigkeit. Kurz \& Kloker [59] untersuchten das Grenzschichtströmungsfeld nahe eines solchen Rauigkeitselementes mithilfe von DNS und verglichen seinen Einfluss mit und ohne Querströmung. Dabei fanden sie eine ähnliche Situation in beiden Fällen, wobei aber der Hufeisenwirbel in dem Fall mit Querströmung eine deutliche Asymmetrie stromab des Rauigkeitselements aufwies. Eine Seite des Hufeisenwirbels wird durch die Strömung unterstützt 


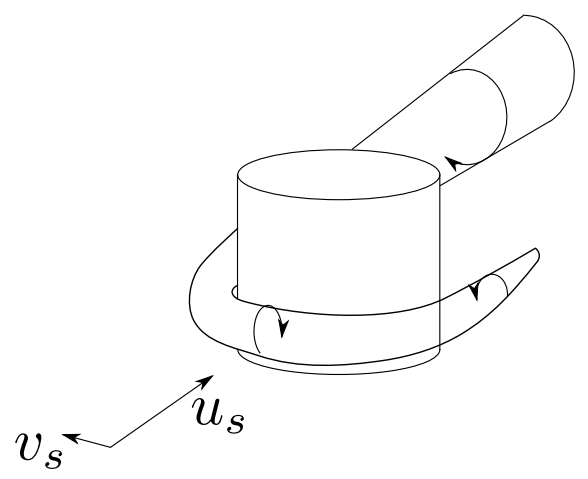

Abbildung 2.5.1: Schematische Darstellung der Entstehung eines Querströmungswirbels stromab eines isolierten zylindrischen Rauigkeitselementes, nach Erkenntnissen von Kurz \& Kloker [59]

und entwickelt sich stromab zu einem stationären elementaren Querströmungswirbel mit passendem Drehsinn, während die andere Seite schnell an Stärke abnimmt und stromab verschwindet. Diese Art der Anregung eines elementaren Querströmungswirbels ist in Abbildung 2.5.1 skizziert. Eine spannweitig periodische Anordnung solcher zylindrischer Rauigkeitselemente eignet sich also zur Anregung einer stationären QSI-Mode. Die entscheidenden Parameter im Rezeptivitätsprozess sind die Form, Höhe, Durchmesser, Position und Verteilung der Einzelrauigkeiten und wurden unter anderem von Müller \& Bippes [77], Radeztsky et al. [84], Deyhle \& Bippes [21], Reibert [88] und Reibert \& Saric [89] untersucht, siehe auch Abschnitt 2.3.

\subsubsection{Lokale Heizung}

Die künstliche Anregung stationärer QSI durch lokale und spannweitig periodische Wandheizung wurde bereits prinzipiell durch Berger [6] untersucht. Im ursprünglichen Prinzipexperiment der schiebenden ebenen Platte untersuchte er einen Heizaktuator, der als Vorbild für den in dieser Arbeit entwickelten Aktuator diente. Der Heizaktuator bestand aus spannweitig periodisch angeordneten Heizelementen, welche jeweils lokal die Wandtemperatur erhöhten. Zur Erläuterung des physikalischen Rezeptivitätsmechanismus einer solchen Aktuierung auf stationäre QSI stützte er sich auf eine Arbeit von Robey [92] zur Anregung von TS-Instabilitäten mit lokaler Wandheizung in einer zweidimensionalen Grenzschicht. Robey bezog sich wiederum auf 
frühere Untersuchungen durch Liepmann et al. [65, 66] sowie Nosenchuck [80], ebenfalls zur Anregung von TS-Instabilitäten.

Berger betrachtete analog zu den Arbeiten der anderen erwähnten Autoren den Reibungsterm in der ersten Grenzschichtgleichung 2.1.8 ohne die dort getroffene Annahme konstanter Viskosität:

$$
\frac{1}{\rho} \frac{\partial \tau_{x z}}{\partial z}=\frac{1}{\rho} \frac{\partial}{\partial z}\left(\mu \frac{\partial u_{c}}{\partial z}\right)=\frac{1}{\rho} \frac{\partial \mu}{\partial z} \frac{\partial u_{c}}{\partial z}+\frac{\mu}{\rho} \frac{\partial^{2} u_{c}}{\partial z^{2}}
$$

und interpretierte $\frac{1}{\rho} \frac{\partial \mu}{\partial z}$ als durch die Temperaturabhängigkeit der Viskosität getriebene induzierte wandnormale Geschwindigkeit:

$$
\frac{1}{\rho} \frac{\partial \mu}{\partial z}=\frac{1}{\rho} \frac{\partial \mu}{\partial T} \frac{\partial T}{\partial z}=:-w_{\text {ind }}
$$

bzw. in der ersten stationären Grenzschichtgleichung:

$$
\begin{aligned}
u_{c} \frac{\partial u_{c}}{\partial x_{c}}+w \frac{\partial u_{c}}{\partial z} & =-\frac{1}{\rho} \frac{\partial p}{\partial x_{c}}+\frac{1}{\rho} \frac{\partial \mu}{\partial T} \frac{\partial T}{\partial z} \frac{\partial u_{c}}{\partial z}+\frac{\mu}{\rho} \frac{\partial^{2} u_{c}}{\partial z^{2}} \\
u_{c} \frac{\partial u_{c}}{\partial x_{c}}+\left(w+w_{i n d}\right) \frac{\partial u_{c}}{\partial z} & =-\frac{1}{\rho} \frac{\partial p}{\partial x_{c}}+\nu \frac{\partial^{2} u_{c}}{\partial z^{2}}
\end{aligned}
$$

Berger [6] argumentierte, dass die so identifizierte induzierte vertikale Geschwindigkeitskomponente $w_{\text {ind }}$ für die Anregung eines elementaren Querströmungswirbels verantwortlich sei. An beiden Seiten senkrecht zur Hauptströmungsrichtung an der Position der lokalen Wandheizung erzeugt die induzierte Vertikalgeschwindigkeit demnach gegensinnig rotierende Wirbelstörungen, von denen eine aufgrund ihres Drehsinns in der Überlagerung mit der Grundströmung stromab angefacht ist und die andere gedämpft. Die Anregung eines elementaren Querströmungswirbels durch eine induzierte Vertikalgeschwindigkeit ist in Abbildung 2.5.2 skizziert.

Derselbe Mechanismus wird für die künstliche Anregung elementarer Querströmungswirbel mithilfe einer diskreten stationären pneumatischen Aktuierung verantwortlich gemacht [70]. Lohse et al. [69] untersuchten erfolgreich die künstliche Anregung stationärer QSI durch spannweitig periodisch verteiltes diskretes Ausblasen sowie Einsaugen. Beim Einsaugen zeigt die induzierte vertikale Geschwindigkeitskomponente $w_{\text {ind }}$ zur Wand und der Drehsinn der beiden erzeugten Wirbelstörungen ist vertauscht. 


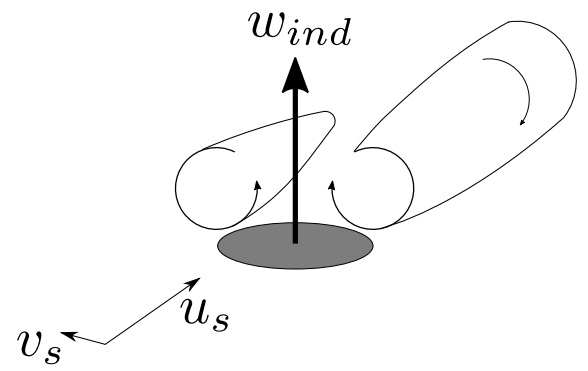

Abbildung 2.5.2: Schematische Darstellung der künstlichen Anregung eines elementaren stationären Querströmungswirbels aufgrund einer induzierten vertikalen Geschwindigkeitskomponente $w_{i n d}$, nach Berger [6]

\subsubsection{Rotationsaktuierung}

Die Strömungen über rotierenden Scheiben sind seit langem Gegenstand der Forschung, einerseits wegen ihrer eigenen technischen Bedeutung, andererseits weil in der Grenzschicht auf einer rotierenden Scheibe Querströmungsinstabilitäten auftreten und die Grenzschicht somit eine einheitliche Modellströmung für die Erforschung querströmungsdominierter Transition darstellt. Für eine Zusammenfassung der Forschung in der Grenzschicht auf einer rotierenden Scheibe siehe [97] und [87]. Ein Vorteil dieser Modellströmung ist, dass für die Strömung über einer ebenen Scheibe, die mit konstanter Drehzahl in sonst ruhendem Fluid rotiert, eine exakte Lösung der Navier-Stokes-Gleichungen existiert. Diese Lösung wurde zuerst von Kármán [52] gefunden und wird auch als Kármán-Strömung bezeichnet. Durch die Reibung wird das Fluid in der Nähe der rotierenden Wand beschleunigt und erhält eine Rotation. Durch die Trägheit wird dabei Material auch in radialer Richtung nach außen transportiert, woraufhin aufgrund der Kontinuität Material ins Innere nachfließen muss. Es entsteht eine axial zur Scheibe gerichtete wandnormale Geschwindigkeitskomponente.

Dem Autor ist keine Untersuchung bekannt, in welcher der Einfluss einer rotierenden Scheibe auf eine über sie strömende laminare Grenzschicht mit nicht verschwindender Haupt- und Querströmungskomponente untersucht wurde, deren Grenzschichtdicke in derselben Größenordnung liegt wie der Radius der Scheibe. Daher soll hier eine Hypothese gemäß einer privaten Mitteilung von S. Hein [42] dargestellt werden, wie eine solche Anordnung zur Anregung eines elementaren Querströmungswirbels in dieser Grenzschicht führen kann. 


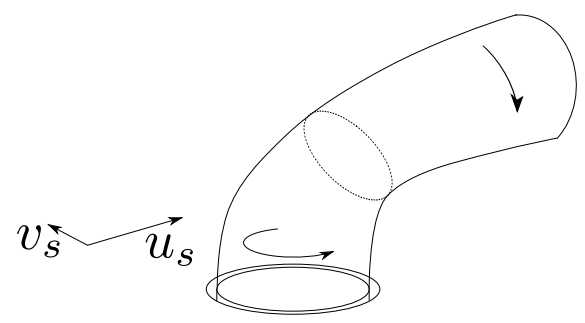

Abbildung 2.5.3: Schematische Darstellung der Hypothese zur Erzeugung eines elementaren Querströmungswirbels mithilfe einer rotierenden Scheibe, nach S. Hein [42]

Wie bei der Kármán-Strömung beschrieben, wird dem Fluid durch die Reibung in der Nähe der Wand eine Rotation aufgeprägt, der sich die ausgeprägte Grenzschichtströmung überlagert. Der Wirbelvektor $\vec{w}=\operatorname{rot} \vec{u}$ der induzierten Wirbelströmung steht senkrecht zur Oberfläche der Scheibe. Wird er durch die überlagerte, verhältnismäßig groß anzunehmende Hauptgeschwindigkeitskomponente $u_{s}$ in eine wandparallele Richtung gekippt, ähnelt die so entstandene Störung stromab in der Grenzschicht bereits einem stationären Längswirbel, aus dessen spannweitig periodischer Anordnung eine stationäre QSI-Mode besteht. Somit würde sich auch eine spannweitig periodische Anordnung solcher Scheiben eignen, um eine stationäre QSI-Mode künstlich anzuregen. Dieser hypothetische Anregungsmechanismus eines elementaren Querströmungswirbels ist in Abbildung 2.5.3 skizziert.

Von den hier vorgestellten Methoden zur künstlichen Anregung stationärer Querströmungsinstabilitäten hätte nach der vorgestellten Hypothese das Ergebnis dieser Form der Anregung die größte Ähnlichkeit zur Störströmung einer QSI-Mode und hätte damit das Potential einer besonders effizienten Anregung. Die direkte Überprüfung der Hypothese entzieht sich aufgrund der geringen räumlichen Ausdehnung und dem geringem Betrag der initialen Störungsgrößen einer experimentellen Untersuchung und liegt nicht im Rahmen dieser Arbeit. Vielmehr dient diese hypothetische Modellvorstellung der Einordnung der experimentellen Ergebnisse, die im Ergebnisteil der Arbeit mit dieser Hypothese verglichen werden. 


\section{Kapitel 3}

\section{Numerische Methoden}

Die im Rahmen dieser Arbeit experimentell untersuchte Strömungssituation um die Konfiguration des neu ausgelegten Prinzipexperiments der schiebenden ebenen Platte in der offenen Messstrecke des Ein-Meter-Windkanals (1MG) (siehe Abschnitt 5.2) ist trotz der großen Spannweite und idealisiert fehlender spannweitiger Gradienten entlang dieser Spannweite durch dreidimensionale Effekte beeinflusst. Eine getreue numerische Nachbildung des Experiments müsste also die dreidimensionale Strömung um die Konfiguration unter Berücksichtigung des Einflusses der Düsengeometrie, der offenen Messstrecke und des Auffangtrichters simulieren, welches im Rahmen dieser Arbeit zu aufwendig war. Stattdessen wurden in unterschiedlichen Stadien der Arbeit zweidimensionale und quasi-dreidimensionale numerische Simulationen der Konfiguration in einer freien Anströmung durchgeführt. Zur Übersicht wird im Folgenden kurz beschrieben, wann welches numerische Verfahren zum Einsatz kam. Danach werden diese Verfahren kurz eingeführt.

Im Auslegungsprozess kam der zweidimensionale Strömungslöser MSES [27, 28, 29, 30] zum Einsatz, da für die Auslegung besonders eine geeignete Druckverteilung entscheidend war und diese als dimensionslose Verteilung $c_{p}\left(x_{c}\right)$ bei unendlich schiebenden Bedingungen nicht vom Schiebewinkel abhängt. Zur Vorbereitung einer Stabilitätsuntersuchung mit der Software NOLOT [45] wurde die Grundströmung in der untersuchten Grenzschicht mithilfe des Grenzschichtlösers COCO [18, 73] bestimmt. Dies geschah im iterativen Auslegungsprozess auf Basis der mit MSES bestimmten Druckverteilung. Die 
Ergebnisse der Stabilitätsuntersuchungen wurden bei der weiteren iterativen Auslegung berücksichtigt, siehe Abschnitt 4.3. Die mit MSES final ausgelegte Konfiguration wurde mithilfe des dreidimensionalen Strömungslösers TAU [34, 35 in quasi-dreidimensionalen Simulationen untersucht, insbesondere mit den im Experiment realisierten Anströmungsbedingungen und dem experimentell bestimmten effektiven Schiebewinkel, siehe Abschnitt 6.1. Auch auf Basis der experimentell bestimmten Druckverteilung und den gegenüber der Auslegung leicht veränderten Anströmbedingungen wurde eine numerische Grundströmung mit COCO bestimmt (zu den experimentellen Anströmbedingungen siehe Abschnitt 6.1.2). Diese numerische Grundströmung wurde ebenfalls mit NOLOT auf Stabilität untersucht (siehe Abschnitt 6.2), um die beobachtete Störströmung mit den gemäß LST-Analyse erwarteten Instabilitäten zu vergleichen. Für alle numerischen Verfahren wurde die jeweils verwendete Netzauflösung basierend auf geeigneten Erfahrungswerten ähnlicher Untersuchungen im DLR gewählt.

\subsection{Berechnung der Grundströmung}

\section{MSES}

Das Softwarepaket MSES, welches von Mark Drela am MIT (Massachusetts Institute of Technology) entwickelt wurde, wurde aufgrund seiner Fähigkeit zum inversen Design [29] im Rahmen der Neuauslegung des Prinzipexperiments zur Anpassung der Druckverteilung auf der Modellplattenoberseite verwendet, siehe Abschnitt 4.3. Es simuliert zweidimensionale Strömungen um Konfigurationen, indem es den reibungsfreien Teil des Strömungsfeldes durch die Euler-Gleichungen beschreibt. Grenzschichten werden durch eine integrale Zweigleichungs-Grenzschicht-Formulierung beschrieben [30]. Die beiden Teile des Strömungsfeldes sind durch die Verdrängungsdicke gekoppelt, indem die Wandstromlinie um die lokale Verdrängungsdicke verschoben wird. Nach der Diskretisierung wird das komplette gekoppelte Gleichungssystem durch eine Newton-Methode gelöst [28].

MSES bietet die Möglichkeit zum inversen Design. Dabei wird nach Vorgabe einer geänderten Druckverteilung an einer Modellkontur diese Kontur entsprechend deformiert, um die Zieldruckverteilung zu erreichen. 


\section{COCO}

COCO ist eine Software zur Lösung der laminaren kompressiblen Grenzschichtgleichungen für stationäre Strömungen um unendlich schiebende Flügel und konische Körper [18, 73]. In seiner Standard-Version bietet COCO an der Wand nur eine adiabate Randbedingung. In einer erweiterten Version der Software [43] kann auch eine Temperaturverteilung als isotherme Randbedingung an der Wand vorgegeben werden, was in Abschnitt 7.3.1 verwendet wird. Des Weiteren gehören Werkzeuge zur Software, die es ermöglichen, eine experimentell ermittelte Wanddruckverteilung als Ausgangspunkt für eine Lösung der Grenzschichtgleichungen zu verwenden.

Im Rahmen des Auslegungsprozesses der experimentellen Konfiguration wurde die Konfiguration mit MSES iterativ so angepasst, dass eine Schritt für Schritt besser geeignete Druckverteilung auf die zu untersuchende Grenzschicht aufgeprägt wird. Dazu wurde eine rein zweidimensionale Umströmung der Konfiguration simuliert. Auf Basis der $c_{p}$-Verteilung wird mit COCO eine unendlich schiebend angeströmte Grenzschicht simuliert (siehe Definition von $c_{p}$ im unendlich schiebenden Fall Gleichung (2.1.13)). Diese Grenzschichtgrundströmung wurde mit NOLOT auf Stabilität untersucht (siehe folgender Abschnitt), um den weiteren iterativen Auslegungsprozess zu den gewünschten Stabilitätseigenschaften lenken zu können. Der Auslegungsprozess wird in Kapitel 4 motiviert und näher beschrieben.

Nach Fertigstellung der finalen Konfiguration und experimenteller Bestimmung der tatsächlich realisierten Strömungsbedingungen wurde mithilfe von COCO auf Basis der experimentell bestimmten Druckverteilung wieder eine Grenzschicht-Grundströmung bestimmt, welche auf Stabilität untersucht wurde, siehe auch die Abschnitte 6.1 und 6.2. Zur Lösung der Grenzschichtgleichungen für die jeweilige Grundströmung wurde das Strömungsfeld an 400 entlang $x_{c}$ verteilten Stationen und 150 wandnormal verteilten Punkten diskretisiert.

\section{TAU}

Zur Vorbereitung der Neuauslegung des Prinzipexperimentes der schiebenden ebenen Platte wurde die stationäre Strömung im ursprünglichen Prinzipexperiment quasi-dreidimensional mit dem Strömungslöser TAU [34, 35] simuliert, siehe Abschnitt 4.1. Des Weiteren wurde auch die stationäre Umströmung der 
finalen Konfiguration quasi-dreidimensional mit TAU untersucht, um die realisierte Wanddruckverteilung und die Strömungsgrößen am Grenzschichtrand im Experiment mit numerischen Ergebnissen zu vergleichen. Dabei wurden die Anströmungsbedingungen variiert, um die experimentellen Ergebnisse möglichst genau nachzuvollziehen, siehe Abschnitt 6.1.

Zunächst wurde um die final ausgelegte Geometrie ein hybrides Rechennetz mithilfe der kommerziellen Software CENTAUR [51] erstellt. Dabei wurde ein kreisförmiger Fernfeldrand mit einem Radius von $500 \cdot c$ vorgesehen, wobei $c=600 \mathrm{~mm}$ der Profiltiefe der Modellplatte entspricht, siehe auch Abschnitt 4.4. Die Oberfläche der Modellplattenoberseite wurde durch 524 entlang $x_{c}$ verteilter Punkte diskretisiert, während das wandnahe Strömungsfeld durch 150 wandnormal verteilte strukturierte Schichten aufgelöst wurde, die über die Grenzschicht hinaus reichen. Die Grenzschichtströmung an der ebenen Platte wurde als rein laminar angenommen. An allen anderen Modellkonturen wurde eine voll turbulente Grenzschichtströmung angenommen. Es kam dabei das Turbulenzmodell nach Spalart und Allmaras [107] zum Einsatz. Da es sich idealisiert um eine unendlich schiebende Strömung handelt, siehe Abschnitt 2.1, wurden spannweitig periodische Randbedingungen gesetzt. Das erzeugte Rechennetz enthält insgesamt etwa 700000 Netzpunkte. Zur Simulation der Strömung um die Konfiguration wurde TAU in der Version 2016.1.0 verwendet. TAU nutzt eine dreidimensionale Finite-Volumen-Methode zur Integration der Reynolds-gemittelten Navier-Stokes-Gleichungen auf hybriden Netzen. Dabei wurden für die wichtigsten Einstellungen die Standardwerte gewählt, sodass ein zentrales Schema für die räumliche und ein explizites Schema für die zeitliche Diskretisierung verwendet wurde.

\subsection{Lineare Stabilitätsuntersuchungen}

Die linearen lokalen Stabilitätsuntersuchungen der Grundströmung wurden mit NOLOT durchgeführt. NOLOT ist eine Software zur Untersuchung linearer nicht-lokaler Stabilität, welche durch das DLR und das „Totalförsvarets forskningsinstitut" (schwedisches Forschungsinstitut für Verteidigung, FOI) entwickelt wurde und welches die kompressiblen parabolisierten Stabilitätsgleichungen (PSE) löst. Gleichzeitig bietet sie aber auch die Möglichkeit für lineare lokale Stabilitätsuntersuchungen, welche im Rahmen dieser Arbeit mit der Parallelströmungsannahme genutzt wurde, siehe auch Abschnitt 2.2. 
Die Funktionsweise der Software und das Lösungsverfahren der Stabilitätsgleichungen wird in [45] beschrieben. Für die im Rahmen dieser Arbeit durchgeführten Stabilitätsuntersuchungen wurde das Strömungsfeld in wandnormaler Richtung mit 300 Punkten diskretisiert und die Stabilität der Grenzschicht an den 400 in Plattentiefenrichtung verteilten Stationen der COCO-Grundströmung untersucht. Als Randbedingung wird angenommen, dass die Geschwindigkeitskomponenten und der Temperaturanteil der Störströmung an der Wand verschwinden. Am oberen Rand des Rechengebiets werden exponentiell mit dem Wandabstand abklingende Störungen angenommen. Für die weitere Vorgehensweise und Ergebnisse siehe Abschnitt 6.2 . 


\section{Kapitel 4}

\section{Die experimentelle Konfiguration}

Die Auslegung der experimentellen Konfiguration für die Untersuchungen dieser Arbeit orientierte sich an dem Prinzipexperiment der schiebenden ebenen Platte mit Verdrängungskörper, wie es im Zeitraum zwischen ca. 1986 [79] und 2001 [1] am DLR Göttingen für grundlegende Untersuchungen des querströmungsdominierten Transitionsprozesses verwendet wurde. Dieses Experiment wird zunächst im folgenden Abschnitt beschrieben. Anhand von Ergebnissen numerischer Untersuchungen zur Strömung in diesem Experiment wird die Zielsetzung der Neuauslegung motiviert. Im weiteren Teil dieses Kapitels folgt die Beschreibung der Vorgehensweise und des Ergebnisses der Neuauslegung. Für weitere Details siehe auch [5].

\subsection{Das ursprüngliche Prinzipexperiment der schiebenden ebenen Platte}

Das ursprüngliche Prinzipexperiment der schiebenden ebenen Platte (siehe Skizzen in Abb. 4.1.1) wurde von Nitschke-Kowsky [79] basierend auf einem Vorschlag von Hirschel und Dallmann [46] ausgelegt und war Grundlage von umfangreichen Untersuchungen am DLR Göttingen zum querströmungsdominierten Transitionsprozess [8, 9, 22, 62, 75, 79], welche das physika- 
lische Verständnis desselben maßgeblich vorantrieben. Ziel der Auslegung war damals, experimentelle Untersuchungen des querströmungsdominierten Transitionsprozesses und insbesondere einen Vergleich mit der Theorie zu ermöglichen. Es war bekannt, dass die Oberflächenkrümmung des umströmten Körpers einen signifikanten Einfluss auf die Stabilität der untersuchten Grenzschicht haben kann, was aber in den damaligen numerischen Verfahren nicht berücksichtigt werden konnte. Dies motivierte maßgeblich die Wahl einer ebenen Platte als umströmtes Modell [79]. Der für Querströmungsinstabilität nötige negative Druckgradient musste daher mittels eines über der Platte angeordneten weiteren Körpers, eines sog. Verdrängungskörpers in einer schiebend angeströmten Anordnung auf die Platte aufgeprägt werden. Die untersuchte Grenzschicht war diejenige an der oberen Plattenoberfläche. Um Querströmungsinstabilität isoliert von Tollmien-Schlichting (TS) -Instabilität zu untersuchen, sollte der Druckgradient entlang der gesamten Plattentiefe stromab der Staulinie negativ sein, was die Grenzschicht bzgl. TS-Instabilität stabilisiert. Die Kontur des Verdrängungskörpers wurde experimentell iteriert bis auf der Plattenoberseite eine geeignete Druckverteilung erreicht wurde, deren Verlauf nahezu entlang der gesamten Profiltiefe eine negative Steigung mit annähernd konstantem Betrag aufwies [79]. Vor dem Verdrängungskörper wurde ein Vorflügel angeordnet, um die Ablösetendenz auf der Oberseite des Verdrängungskörpers zu verringern. Diese experimentelle Konfiguration ist als Querschnittsskizze in Abbildung 4.1.1(a) und als Aufsichtsskizze im EinMeter-Windkanal in Abb. 4.1.1(b) dargestellt.

Die Plattentiefe betrug $c=500 \mathrm{~mm}$ und ihre Dicke hatte in den Arbeiten von Nitschke-Kowsky [79] den Wert $h=30 \mathrm{~mm}$. In den später folgenden Untersuchungen kamen auch Modellplatten mit den Dicken $h=25 \mathrm{~mm}$ und $h=32 \mathrm{~mm}$ zum Einsatz. Um unendlich schiebende Bedingungen anzunähern und somit Vergleiche mit der Theorie unter den in Abschnitt 2.1 beschriebenen Annahmen zu ermöglichen, wurden konturierte Seitenbleche zwischen Verdrängungskörper und Platte mit der Kontur der Grenzschichtrandstromlinie, die sich unter Annahme unendlich schiebender Bedingungen einstellen sollte, eingesetzt. Als typische Anströmungsbedingungen wurde die Anströmgeschwindigkeit $q_{\infty}=19 \mathrm{~m} / \mathrm{s}$ und ein Anströmungsschiebewinkel von $\phi_{\infty}=-45.0^{\circ}$ gewählt.

Für die von Nitschke-Kowsky ausgelegte Konfiguration wurde die Strömung im Rahmen der vorliegenden Arbeit quasi-dreidimensional mit TAU simuliert (siehe Abschnitt 3.1). Müller [76] beschreibt eine gute Übereinstimmung der Strömungsgrößen am Grenzschichtrand der Modellplattenoberseite zwischen 
(a)

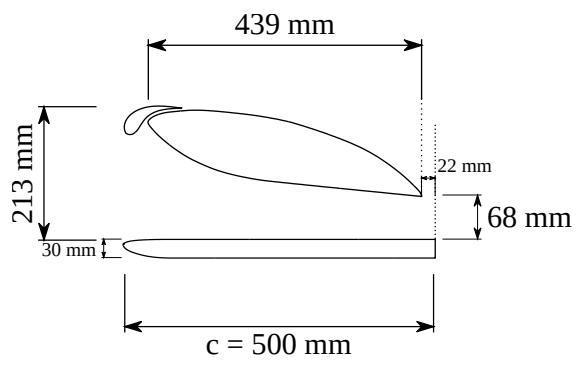

(b)

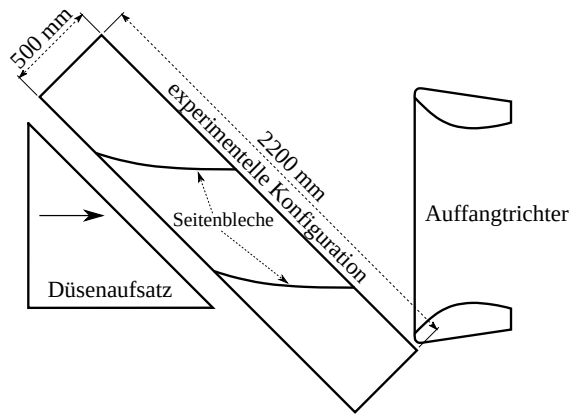

Abbildung 4.1.1: (a) Querschnittskizze der experimentellen Konfiguration im ursprünglichen Prinzipexperiment der schiebenden ebenen Platte nach NitschkeKowsky [79] (b) Aufsichtsskizze der Konfiguration im Ein-Meter-Windkanal nach Müller [76]

dem Experiment und einer numerischen Simulation mit einer freien Anströmung für einen effektiven Schiebewinkel von $\phi_{\text {eff }}=-42.5^{\circ}$. Die Abweichung des effektiven Schiebewinkels vom geometrischen Schiebewinkel ist der Tatsache geschuldet, dass in der numerischen Simulation der dreidimensionale Einfluss der offenen Messstrecke nicht berücksichtigt wird (siehe analog für die neu ausgelegte Konfiguration auch die Diskussion in Abschnitt 6.1). Für die Simulation der Umströmung der ursprünglichen Konfiguration mit TAU in dieser Arbeit wurde also $q_{\infty}=19 \mathrm{~m} / \mathrm{s}$ mit demselben effektiven Schiebewinkel gewählt. Um die experimentell bestimmte $x_{c}$-Position der Staulinie der früheren experimentellen Arbeiten nachzubilden, wurde in der TAUSimulation ein effektiver Anstellwinkel von $\alpha=-4^{\circ}$ gewählt. In Abbildung 4.1.2(a) sind zur Visualisierung der Umströmung der Konfiguration Werte der $u_{c}$-Komponente aus dieser numerischen Strömungssimulation als farbliches Isokonturendiagramm dargestellt. Dabei erkennt man unter anderem eine Ablöseblase auf der Oberseite des Verdrängungskörpers sowie an der Hinterkante der Modellplatte.

In Abbildung 4.1.2(b) ist der Druckbeiwert $c_{p}$ aus der TAU-Simulation auf der Oberseite der Modellplatte im Vergleich mit unveröffentlichten Messergebnissen von T. Lerche [63] dargestellt. Dabei erkennt man eine gute Übereinstimmung und die beinahe konstante Beschleunigung entlang eines Großteils der Profiltiefe. Mithilfe der Software COCO [18] (siehe Abschnitt 
(a)

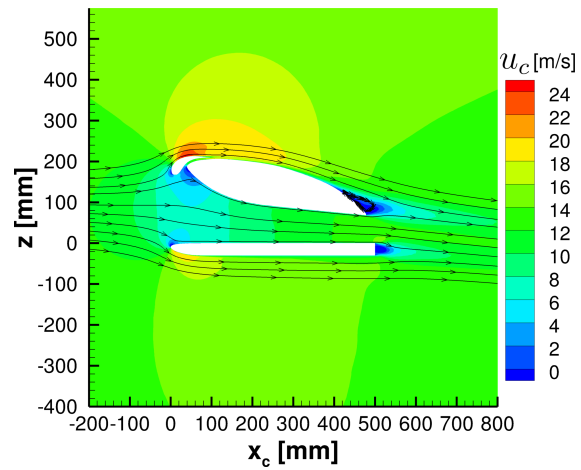

(b)

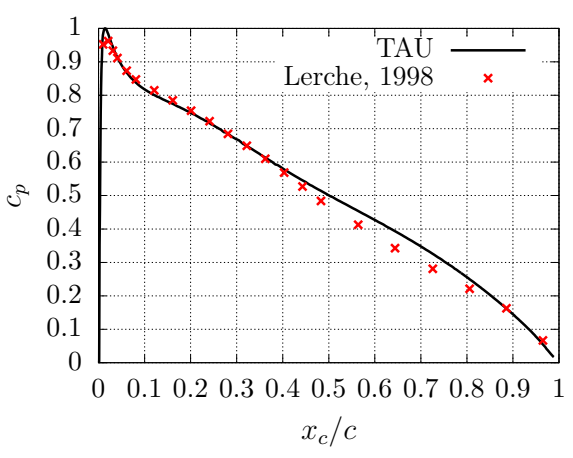

Abbildung 4.1.2: (a) Numerisch simulierte Umströmung der Konfiguration gemäß der TAU-Simulation (b) Vergleich der Druckverteilung gemäß der TAU-Simulation mit Messergebnissen von Lerche [63]

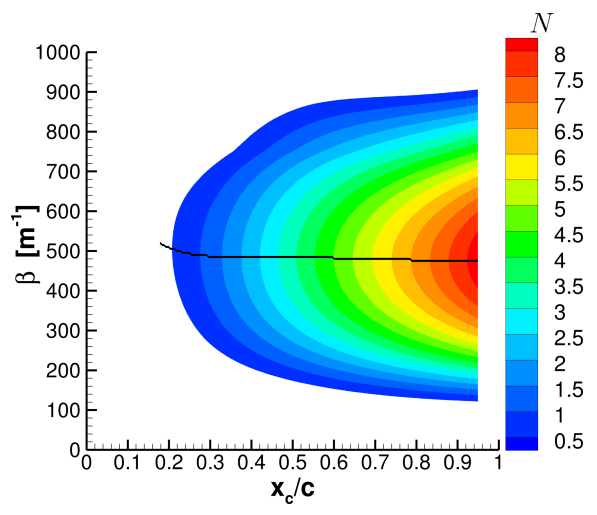

Abbildung 4.1.3: N-Faktoren stationärer Querströmungsinstabilitäten im ursprünglichen Prinzipexperiment der schiebenden ebenen Platte

3.1) wurde auf Grundlage dieser Druckverteilung eine Grenzschichtströmung simuliert, welche mit NOLOT [45] (siehe Abschnitt 3.2) linear und lokal auf Stabilität untersucht wurde. In Abbildung 4.1.3 sind die resultierenden NFaktoren stationärer Querströmungsinstabilitäten abhängig von der Profiltiefenkoordinate $x_{c} / c$ und der spannweitigen Wellenzahl $\beta$ als Isokonturen dargestellt. Des Weiteren ist eine Linie mit eingezeichnet, welche die Wellenzahl der Moden verbindet, die an der jeweiligen $x_{c}$-Position den höchsten NFaktor erreichen. Dabei erkennt man, dass entlang der gesamten Plattentiefe 
die QSI mit nahezu derselben spannweitigen Wellenzahl integral am stärksten angefacht sind, was für die Funktionsweise der UFD-Methode keine idealen Voraussetzungen bietet (siehe Abschnitt 2.4).

Für die Untersuchungen zur UFD-Methode im Rahmen des Projektes AKSA („Aktive Kontrolle von Störungen in der Aerodynamik“) und im Rahmen dieser Arbeit wurde daher bei der Neuauslegung der experimentellen Konfiguration die Kontur des Verdrängungskörpers grundlegend verändert, um eine veränderte Druckverteilung auf die Grenzschicht der Modellplattenoberseite aufzuprägen und für geeignetere Stabilitätseigenschaften zu sorgen. Diese Neuauslegung wird in den folgenden Abschnitten beschrieben.

\subsection{Zielsetzung der Neuauslegung}

Wie im ursprünglichen Prinzipexperiment der schiebenden ebenen Platte sollte eine bzgl. Querströmungsinstabilitäten instabile 3D-Grenzschicht untersucht werden, deren Entwicklung bis zum finalen laminar-turbulenten Umschlag und darüber hinaus detailliert untersucht werden kann. Die Strömung im Experiment sollte möglichst gut unendlich schiebende Bedingungen annähern, siehe Abschnitt 2.1. Instationäre Ablösegebiete sollten vermieden werden, um eine möglichst stationäre Umströmung der Konfiguration zu realisieren.

Rasterförmige Hitzdrahtmessungen und andere Messtechniken werden durch eine ebene Modelloberfläche stark vereinfacht. Daher fiel in der vorliegenden Arbeit wie im ursprünglichen Prinzipexperiment die Wahl auf eine ebene Modellplatte, auf deren Oberseite sich die zu untersuchende Grenzschicht ausbildet.

Um Querströmungsinstabilität möglichst isoliert untersuchen zu können, war auch die Unterdrückung von Tollmien-Schlichting-Instabilitäten eine Anforderung an die Neuauslegung, was durch einen entlang nahezu der gesamten Profiltiefe der Modellplatte negativen und betragsmäßig hinreichend großen Druckgradienten erreicht wird. Dieser Druckgradient wird wie im ursprünglichen Prinzipexperiment mithilfe eines Verdrängungskörpers auf die Modellplattenoberseite aufgeprägt. Als geometrischer Schiebewinkel der Konfiguration wurde wie im ursprünglichen Prinzipexperiment $\phi_{g}=-45.0^{\circ}$ gewählt. 
Des Weiteren sollten Untersuchungen zur Transitionsbeeinflussung mittels der UFD-Methode ermöglicht werden. Wie in Abschnitt 2.3 und Abschnitt 2.4 beschrieben, wird bei der UFD-Methode eine kurzwelligere stationäre Primärinstabilität als die natürlich integral angefachteste angeregt, um das Transitionsszenario zu beeinflussen. In Abschnitt 2.4 wird zudem die Anforderung beschrieben, dass diese künstlich anzuregende stationäre Kontrollmode im vorderen Bereich der Modellgrenzschicht ausreichend stark angefacht sein muss. Gleichzeitig sollte sie aber nicht so stark integral angefacht sein, dass sie selbst den laminar-turbulenten Umschlag auslöst. Wie in Abschnitt 4.1 beschrieben, führte der Druckgradient im ursprünglichen Prinzipexperiment mit nahezu konstanter negativer Steigung entlang der gesamten Profiltiefe der Modellplatte dazu, dass nahezu entlang der gesamten Profiltiefe dieselbe Mode stationärer QSI die jeweils angefachteste Mode war.

Die im vorigen Absatz beschriebenen Anforderungen können durch einen Druckgradienten erreicht werden, der im vorderen Bereich des Modells eine starke und im Verlauf stromab eine schwächere Beschleunigung aufweist. Diese Form des Druckgradienten ist im Rahmen dieser Arbeit eine akademisch motivierte Zielsetzung, sie ähnelt aber auch derjenigen an realistischen schiebenden Tragflügeloberseiten, zumindest bis zur Profiltiefe des Druckminimums. Des Weiteren muss die Grenzschichtdicke ausreichend groß sein, um die Grenzschicht mithilfe rasterförmiger Hitzdrahtanemometrie räumlich ausreichend hochaufgelöst untersuchen zu können. Gemeinsam mit der Anforderung, welche die spannweitige Wellenlänge der anzuregenden Instabilitäten an die konstruktive Realisierung der Aktuatorik stellt, schränkt dies die Reynoldszahl der Anströmung und damit die verwendete Anströmgeschwindigkeit bzgl. höherer Werte ein.

\subsection{Vorgehensweise der Neuauslegung}

Die Anforderung, den laminar-turbulenten Umschlag stromab bis zum turbulenten Zustand untersuchen zu können, impliziert in Abhängigkeit von der Anfachung der beteiligten Instabilitäten die Wahl einer ausreichend hohen Profiltiefen-Reynoldszahl. Um bei fester Anströmgeschwindigkeit eine höhere Profiltiefen-Reynoldszahl zu erreichen bzw. eine bestimmte Reynoldszahl bei einer niedrigeren Anströmgeschwindigkeit zu erreichen, wurde die Profiltiefe der Modellplatte von $500 \mathrm{~mm}$ auf $c=600 \mathrm{~mm}$ erhöht. Bei dem gewählten geometrischen Schiebewinkel wird nahezu die gesamte Länge der offenen Mess- 
strecke des Ein-Meter-Kanals ausgenutzt, wodurch eine weitere Erhöhung der Profiltiefe unmöglich ist.

Die Software MSES [30] bietet die Möglichkeit der inversen Konturanpassung gemäß der Vorgabe einer Zieldruckverteilung an derselben Kontur, siehe auch Abschnitt 3.1. Für eine Druckverteilung mit den im Abschnitt 4.2 beschriebenen Eigenschaften war vor allem eine neue Kontur des Verdrängungskörpers nötig. Da es Indizien dafür gab, dass auf der Verdrängungskörperoberseite im ursprünglichen Prinzipexperiment eine Ablösung stattfand, wurde zunächst von Stefan Hein in einer numerischen Vorstudie im Rahmen eines EU-Projekts die Oberseite des ursprünglichen Verdrängungskörpers manuell derart verändert, dass der statische Druckanstieg zur Hinterkante weniger steil verlief und das Profil insgesamt schlanker wurde. Diese Kontur war Ausgangspunkt der folgenden Konturanpassung mit MSES im Rahmen dieser Arbeit. Für die dabei durchgeführten Strömungssimulationen wurde der manuell angepasste Verdrängungskörper so gestreckt, dass seine Profiltiefe die der Modellplatte übertraf. Die Vorderkante des Verdrängungskörpers wurde im Gegensatz zum ursprünglichen Prinzipexperiment stromauf der Vorderkante der Modellplatte angeordnet. Damit wurde der Einfluss des Verdrängungskörpers auf die Druckverteilung im Nasenbereich der Modellplatte verstärkt. Der vertikale Abstand des Verdrängungskörpers zur Modellplatte wurde wie im ursprünglichen Prinzipexperiment gewählt. Der Vorflügel wurde in Relation zum Verdrängungskörper ebenfalls identisch zum ursprünglichen Prinzipexperiment angeordnet.

Die inverse Konturanpassung mit MSES hatte nur bei kleinen Differenzen zwischen der Zieldruckverteilung und der jeweils aktuellen Druckverteilung Erfolg. Auch deshalb wurde der Prozess iterativ durchgeführt. In jeder Iteration wurde zunächst eine gegenüber der aktuellen Druckverteilung nur leicht veränderte Zieldruckverteilung an der Verdrängungskörperunterseite vorgegeben, woraufhin die Kontur der Unterseite durch MSES entsprechend angepasst wurde. Die Eigenschaften der resultierenden Druckverteilung auf der Modellplattenoberseite wurden mit den gewünschten Eigenschaften verglichen, um im nächsten Schritt wieder die Zieldruckverteilung an der Verdrängungskörperunterseite leicht anzupassen. Da die Verdrängungskörperunterseite und die Modellplattenoberseite einen Kanal bilden, besteht ein enger Zusammenhang zwischen den Druckverteilungen an beiden Oberflächen.

Wenn nach einigen solchen Iterationen eine signifikante Änderung der Druckverteilung an der Modellplattenoberseite erreicht wurde, wurde mithilfe des 
Grenzschichtlösers COCO [18] mit dieser Druckverteilung eine Lösung der Grenzschichtgrundströmung erzeugt. Diese wurde dann mit NOLOT [45] auf Stabilität untersucht. Wie im vorigen Abschnitt beschrieben wurde, soll die auszuwählende Kontrollmode im vorderen Bereich der Modellplatte ausreichend stark angefacht sein. Als Kontrollmode wurde jeweils die stationäre QSI-Mode betrachtet, deren spannweitige Wellenlänge $\lambda$ wie in der Veröffentlichung von Saric [95] zwei Dritteln der Wellenlänge der als natürlich dominant erwarteten Mode entsprach (siehe auch Abschnitt 2.4.1). Als natürlich dominante Mode wurde diejenige betrachtet, die am weitesten stromauf einen als kritisch angenommenen N-Faktor erreicht und am wahrscheinlichsten für den laminar-turbulenten Umschlag verantwortlich ist. Als kritischer N-Faktor wurde hierbei $N_{\text {crit }}=9$ ausgewählt. Bei der weiteren iterativen Anpassung der Druckverteilung wurde berücksichtigt, dass die Kontrollmode ausreichend früh einen höheren N-Faktor erreicht als die natürlich dominante Mode, um zu einer Vordeformation der spannweitig gemittelten Grenzschichtströmung führen zu können. Gleichzeitig sollte ihr N-Faktor aber stromab wieder abnehmen, um nicht selber zur Transition zu führen.

Nach diesem iterativen Prozess wurde die endgültige Position des Vorflügels in Relation zum Verdrängungskörper mithilfe von Parameterstudien in TAU angepasst. Dabei wurde der Abstand des Vorflügels zum Verdrängungskörper in Profiltiefen- und vertikaler Richtung variiert und der Einfluss auf die Druckverteilung bzgl. der Ziele der Neuauslegung bewertet. Im Rahmen der begleitenden Stabilitätsuntersuchungen besaß die natürlich dominante Mode eine spannweitige Wellenlänge von $9 \mathrm{~mm}$ und als Kontrollmode wurde jene mit einer spannweitigen Wellenlänge von $\lambda=6 \mathrm{~mm}$ bzw. einer spannweitigen Wellenzahl von $\beta=1047.2 \mathrm{~m}^{-1}$ ausgewählt.

\subsection{Das neu ausgelegte Prinzipexperiment der schiebenden ebenen Platte}

Die resultierende Konfiguration als Ergebnis der in Abschnitt 4.3 beschriebenen Neuauslegung ist als Querschnittskizze in Abbildung 4.4.1(a) und als Aufsichtsskizze in Abb. 4.4.1(b) dargestellt.

Darin erkennt man die ebene Modellplatte mit einer Profiltiefe von $c=$ $600 \mathrm{~mm}$ und einer Dicke von $32 \mathrm{~mm}$. Sie wurde aus einer Aluminium-Legierung mithilfe von CNC-Fräsen gefertigt. Für die Nasenkontur der Modellplatte wurde wie im ursprünglichen Prinzipexperiment das Clark-Y-Profil gewählt, 
(a)

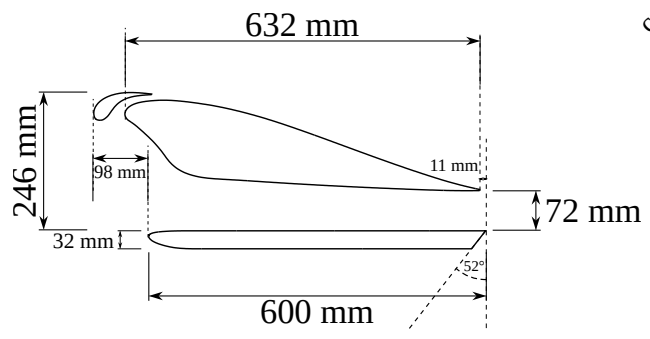

(b)

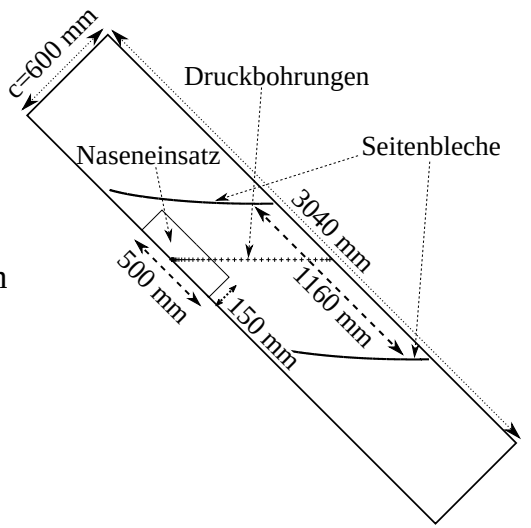

Abbildung 4.4.1: (a) Querschnittsskizze der Konfiguration des neu ausgelegten Prinzipexperiments der schiebenden ebenen Platte (b) Aufsichtsskizze der Modellplatte

dessen Druckseite nach oben ausgerichtet ist. Die Druckseite dieses Profils führt die Kontursteigung stetig von der Vorderkante in $x_{c}$-Richtung auf eine Ebene. Der ebene Bereich der Oberseite erstreckt sich über die Profiltiefe $0.1<x_{c} / c<1$. Als Aufnahmemöglichkeit für Naseneinsätze ist im Nasenbereich der Modellplatte ein Bereich von $500 \mathrm{~mm}$ spannweitiger Breite und $150 \mathrm{~mm}$ Tiefe in $x_{c}$-Richtung ausgespart, welcher in der Abbildung 4.4.1(b) eingezeichnet ist. Am Übergang zwischen dem Naseneinsatz und der restlichen Modellplatte sollte eine Stufe möglichst vermieden werden, da diese eine Störung der Grenzschichtströmung darstellen würde. Durch geeignet angeordnete Schrauben wurde bei jedem Einbau eines Naseneinsatzes sorgfältig ein bündiger Übergang eingestellt.

Für die Experimente im Rahmen dieser Arbeit kamen drei solcher Naseneinsätze zum Einsatz. Neben den in den Abschnitten 4.5.1 und 4.5.2 näher beschriebenen Aktuatoreinsätzen wurde ein Naseneinsatz mit 21 Druckbohrungen zur Messung des Wanddruckes gefertigt, siehe auch Abschnitt 5.3. Dieser Naseneinsatz wurde wie die Modellplatte selbst aus einer AluminiumLegierung mithilfe von CNC-Fräsen gefertigt. Er wird zur grundlegenden Charakterisierung der transitionellen Grenzschichtströmung im Referenzfall verwendet, siehe Kapitel 6. Im restlichen Profiltiefenbereich der Modellplatte, also für $x_{c}>150 \mathrm{~mm}$ bzw. $x_{c} / c>0.25$, sind weitere 19 Druckbohrungen 
eingelassen. Sämtliche Druckbohrungen sind entlang einer Geraden mit dem Winkel $45^{\circ}$ zur Vorderkante und damit parallel zur Anströmungsrichtung angeordnet. Alle Druckbohrungen besitzen einen Durchmesser von $0.2 \mathrm{~mm}$. Ihre Profiltiefenpositionen sind im Anhang tabellarisch aufgeführt, siehe Tabelle A.1.1. Die Oberfläche der Modellplatte und des Naseneinsatzes mit Druckbohrungen wurde geschliffen und wies eine aus mehreren Messungen gemittelte Rautiefe von $\bar{R}_{z}=3.0(5) \mu \mathrm{m}$ und einen gemittelten Mittenrauwert von $\bar{R}_{a}=0.5(1) \mu \mathrm{m}$ auf, wobei der angegebene Fehler der Standardabweichung über die durchgeführten Messungen entspricht.

Die Hinterkante der Modellplatte wurde mit einer Fase mit einem Winkel von $52^{\circ}$ versehen. Über der Modellplatte ist der Verdrängungskörper angeordnet, sodass ein Kanal zwischen ihm und der Modellplatte gebildet wird, welcher an der Hinterkante seinen engsten Querschnitt mit einem vertikalen Abstand von $72 \mathrm{~mm}$ erreicht. Am Verlauf des Querschnitts dieses Kanals erkennt man qualitativ den Verlauf des Druckgradienten in $x_{c}$-Richtung, welchen der Verdrängungskörper auf der Oberseite der Modellplatte aufprägt. Im vorderen Bereich der Platte verringert sich dieser Querschnitt in einem vergleichsweise kurzen $x_{c}$-Bereich stark und im weiteren Verlauf nur noch schwach, was in der Grenzschichtströmung auf der Modellplatte eine starke Beschleunigung gefolgt von einer schwächeren Beschleunigung bewirkt. Der Verdrängungskörper wurde aus einem Polystyrolkern in Positivbauweise mit einem Holm aus UHMKohlefaser (UHM=,Ultra-Hochmodul“, wobei ein hohes Elastizitätsmodul gemeint ist) und einer Kohlefaserschale gefertigt. Der Vorflügel, welcher stromauf und leicht oberhalb des Verdrängungskörpers angeordnet ist, wurde ebenfalls als ein Kohlefaser-Hartschalenflügel mit einem Holm aus UHMKohlefaser gefertigt. Dies wurde allerdings nicht in Positivbauweise, sondern mithilfe einer CNC-gefrästen Negativform ausgeführt.

Die Konfiguration bietet mit einer Spannweite von $3000 \mathrm{~mm}$ die Möglichkeit, den umströmten Bereich spannweitig in der offenen Messstrecke des 1MG zu verschieben. Der Verdrängungskörper und der Vorflügel werden an den beiden spannweitigen Enden der ebenen Platte fixiert (siehe auch Abb. 5.2.2). Bei der Auslegung und Materialwahl wurde auf eine größtmögliche spannweitige Steifigkeit beider Körper geachtet, um Vibrationen und damit zeitliche Schwankungen der Wanddruckverteilung auf der Modellplatte zu vermeiden. Die Modellplatte wird ebenfalls an ihren spannweitigen Enden fixiert, aber zusätzlich spannweitig mittig von unten mit einer Säule gestützt. Die Fixierung und Positionierung in der Messstrecke wird durch ein Gerüst aus X95-Bauteilen gewährleistet. X95 bezeichnet dabei ein System aus Profilen, 
Reitern, Winkel- und anderen Bauteilen, welches oft für optische und andere experimentelle Aufbauten verwendet wird.

Des Weiteren erkennt man in der Aufsichtsskizze in Abbildung 4.4.1(b) die konturierten Seitenbleche, welche den Messbereich in einem spannweitigen Abstand von $1160 \mathrm{~mm}$ zueinander spannweitig begrenzen. Bereits im ursprünglichen Prinzipexperiment wurden solche konturierten Seitenbleche verwendet, um spannweitige Gradienten der Strömungsgrößen aufgrund des Einflusses der offenen Messstrecke zu verringern [21, 62, 76, 79]. Sie besitzen in der $x_{c}-y_{c}$-Ebene die Kontur der gekrümmten Grenzschichtrandstromlinie gemäß der finalen Druckverteilung der Neuauslegung und der Annahme unendlich schiebender Bedingungen (siehe Gleichungen 2.1.13 und 2.1.15). Projiziert auf die $x_{c^{-}} z$-Ebene besitzen sie die Kontur, welche dem oben beschriebenen Kanal zwischen Verdrängungskörper und Modellplatte entspricht. Die Seitenbleche bestehen aus Aluminiumblech mit einer Stärke von $2 \mathrm{~mm}$ und sind an einer passend konturierten Rippe aus Holz fixiert. Diese Rippe befindet sich jeweils an der dem Messbereich abgewandten Seite und bringt das Blech in die gewünschte Kontur. In Plattentiefenrichtung decken die Seitenbleche einen Bereich von $0.05<x_{c} / c<0.98$ ab. Damit befinden sie sich stromab des Bereichs nahe der Anlegelinie, in dem die Stromlinien noch sehr stark gekrümmt sind.

In Abschnitt 6.1 wird geprüft, inwiefern die Strömung im neu ausgelegten Prinzipexperiment die Ziele der Neuauslegung erfüllt. Die Stabilitätseigenschaften einer Grenzschichtgrundströmung, welche auf Basis der experimentell bestimmten Druckverteilung numerisch mit COCO simuliert wurde, werden in Abschnitt 6.2 beschrieben. In Abschnitt 6.3 wird dann überprüft, ob die im Experiment beobachtete Störströmung mithilfe der Stabilitätsergebnisse nachvollzogen werden kann und insbesondere ob sie die Ziele der Neuauslegung erfüllt. Eine Fotografie der experimentellen Konfiguration im 1MG ist in Abbildung 5.2.2(a) bei der Beschreibung des Windkanals zu sehen.

\subsection{Aktuatoren}

Wie in Abschnitt 2.4 beschrieben, ist für die Transitionsverzögerung mithilfe der UFD-Methode die gezielte künstliche Anregung einer stationären Instabilitätsmode mit einer wohldefinierten spannweitigen Wellenlänge und Amplitude nötig. Wie in Abschnitt 4.3 beschrieben, wurde für diese Kontrollmode eine feste spannweitige Wellenlänge $\lambda=6 \mathrm{~mm}$ ausgewählt, welche 
einer spannweitigen Wellenzahl von $\beta=\frac{2 \pi}{\lambda}=1047.2 \mathrm{~m}^{-1}$ entspricht. Für die künstliche Anregung wurden im Rahmen dieser Arbeit zwei Aktuatoren entwickelt und jeweils als eigener Naseneinsatz für die Modellplatte aufgebaut. Der Aufbau dieser Aktuatoren wird im Folgenden beschrieben.

\subsubsection{Heizaktuator}

Eine Möglichkeit, stationäre Querströmungsinstabilitäten anzuregen, ist eine stationäre spannweitig periodische Heizung der Modelloberfläche, wie Berger zeigen konnte [6]. Er verwendete ein Platinenätzverfahren, um eine Reihenschaltung von Leiterbahnen mit geringer Querschnittsfläche und Leiterbahnen mit größerer Querschnittsfläche auf einer konventionellen Platine anzuordnen. Die Leiterbahnen mit relativ geringer Querschnittsfläche besitzen einen größeren Ohmschen Widerstand als die Leiterbahnen mit größerer Querschnittsfläche und heizen sich somit bei elektrischem Stromfluss stärker auf. Durch eine spannweitig periodische Anordnung der so erzeugten lokalen Heizelemente konnte er eine spannweitig periodische stationäre Störung in die Strömung einbringen und somit stationäre Querströmungsinstabilitäten mit definierter Wellenlänge anregen. Die konventionelle Platine wurde von Berger bei $x_{c} / c=0.14$ in den bereits ebenen Teil der Modellplatte und nicht im Bereich der gekrümmten Nasenkontur eingelassen. Die Kupferleiterbahnen besaßen eine Höhe von $18 \mu \mathrm{m}$ und stellten damit eine signifikante Rauigkeit dar.

Der Transitionsprozess ist gegenüber der Anregung stationärer QSI am empfindlichsten in der Nähe des Neutralpunktes der anzuregenden Mode [20, 84]. Um eine möglichst effiziente Anregung der stationären Kontrollmode zu erreichen und eine möglichst lange Strecke der linearen Anfachung zu nutzen, sollte die Heizaktuierung im Rahmen dieser Arbeit möglichst nahe am Neutralpunkt dieser Mode möglich sein. Die Position des Neutralpunktes war aufgrund der LST-Untersuchungen zur Neuauslegung bekannt und hat den Wert $x_{c} / c=0.05$. Diese Position liegt damit im Bereich der gekrümmten Nasenkontur der Modellplatte. Daher wurde ein flexibles Platinensubstrat gewählt, auf dem die Leiterbahnen wie schon bei Berger mithilfe eines Ätzverfahrens freigestellt wurden. Die Dicke des flexiblen Substrats betrug $50 \mu \mathrm{m}$, die Höhe der Kupferelemente auf dem Substrat hatte einen Wert von etwa $35 \mu \mathrm{m}$. Im Folgenden wird für das flexible Substrat mit den Kupferelementen auch der Begriff Heizaktuatorfolie verwendet. Für den Heizaktuator wurde ein spezieller Naseneinsatz mit einer Tasche gefertigt, in der die Heizaktuatorfolie 


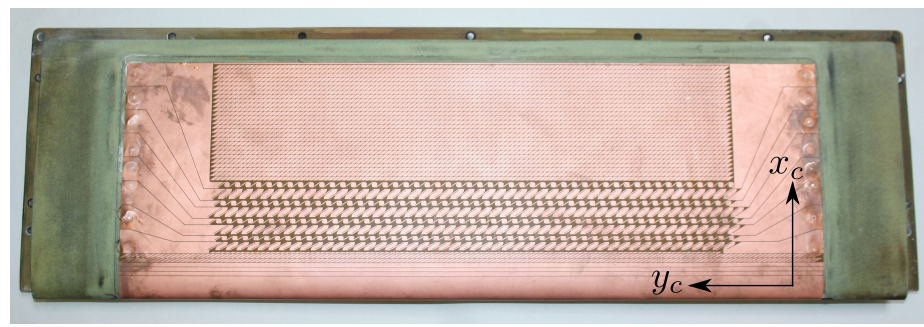

Abbildung 4.5.1: Fotografie der auf dem Heizaktuator-Naseneinsatz fixierten Heizaktuatorfolie vor Auftragen des Epoxid-Füllmaterials

mithilfe doppelseitigen Klebebandes fixiert wurde. Die Tiefe der Tasche war dabei so gewählt, dass die Kupferelemente der eingeklebten Heizaktuatorfolie bündig zur restlichen Oberfläche des Naseneinsatzes abschließen. Um an dem stromauf gelegenen Übergang der restlichen Oberfläche zur Heizaktuatorfolie eine Stufe und damit zusätzliche Störungen der Strömung zu vermeiden, wurde die Folie um die Nase geschlagen und bedeckte auch auf der Modellunterseite noch einen gewissen Bereich. Unter der Tasche für die Heizaktuatorfolie wurde ein Hohlraum für die elektrischen Verbindungen vorgesehen.

Als Material für diesen speziellen Naseneinsatz wurde „Necuron 702“-Epoxidmaterial verwendet, da es im Vergleich zu Aluminium eine deutlich geringere Wärmeleitfähigkeit aufweist, aber mit $415 \mathrm{~K}$ eine hohe Wärmeformbeständigkeitstemperatur gemäß ISO 75 besitzt. Eine geringe Wärmeleitfähigkeit ist von Vorteil, da für die künstliche Anregung von stationären QSI spannweitig periodisch möglichst große Temperaturunterschiede erzeugt werden müssen, siehe Abschnitt 2.5.2. Der Heizaktuator-Naseneinsatz mit fixierter Heizaktuatorfolie ist als Fotografie in Abbildung 4.5.1 dargestellt.

Die Anordnung der Kupferflächen auf der Heizaktuatorfolie ist in Abbildung 4.5.2 dargestellt. Die vertikale Achse der Abbildung entspricht im fixierten Zustand auf dem Modell der Profiltiefenrichtung entlang $x_{c}$. Die Heizelemente geringen Querschnitts und Leitelemente größeren Querschnitts sind in Reihe geschaltet. Eine solche Reihenschaltung wird im Folgenden als Heizreihe bezeichnet. Der spannweitige Abstand der Heizelemente entspricht der spannweitigen Wellenlänge der anzuregenden stationären QSI, $\lambda=6 \mathrm{~mm}$. Durch die Reihenschaltung ist es in dieser technischen Realisierung der Störungsanregung nur möglich, Instabilitäten mit dieser festen Wellenlänge anzuregen. Bei einzelner Kontaktierung der Heizelemente in einem zukünftigen Heizak- 


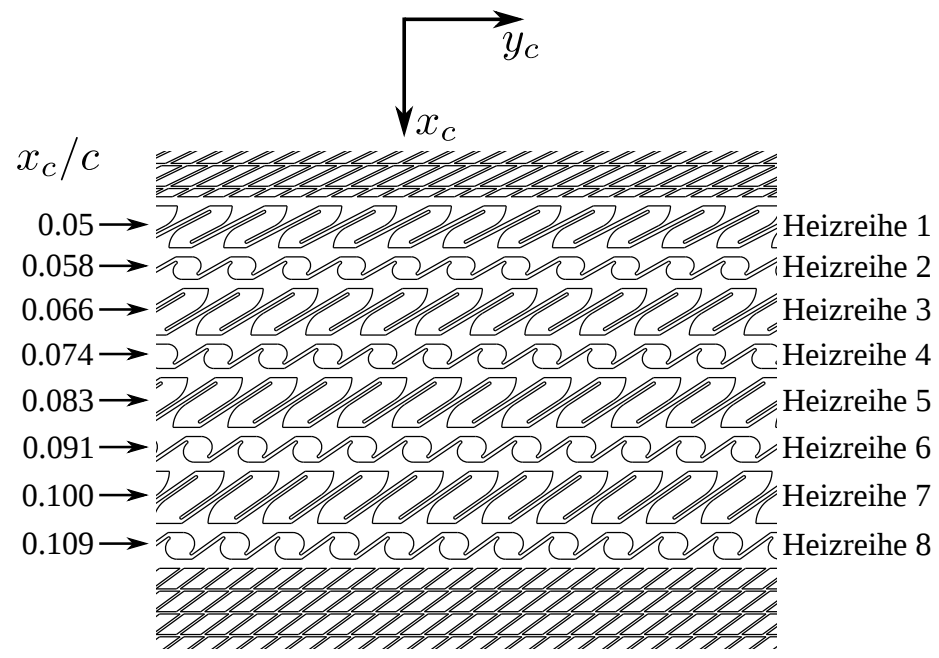

Abbildung 4.5.2: Ausschnitt der Kupferflächen auf der Heizaktuatorfolie.

tuator wäre aber eine Anregung mit spannweitigen Wellenlängen möglich, die ganzzahligen Vielfachen des spannweitigen Abstands der Heizelemente entsprechen. Eine Heizreihe erstreckt sich über eine Spannweite von ca. $300 \mathrm{~mm}$. Um den Einfluss der Aktuierungsposition auf die Effizienz der Anregung untersuchen zu können, wurden acht in Profiltiefenrichtung aufeinanderfolgende Heizreihen auf der Heizaktuatorfolie angeordnet. Der lokale Schiebewinkel $\phi$ der Heizelemente richtet sich nach der Orientierung der Wirbelachse der Kontrollmode an der jeweiligen mittleren Profiltiefenposition. Diese Orientierung der Kontrollmoden-Wirbelachsen wurde aus Stabilitätsuntersuchungen gewonnen. Am jeweiligen spannweitigen Ende einer solchen Heizreihe wird ein elektrischer Kontakt in den darunter liegenden Hohlraum geführt, sodass ein elektrischer Strom mit konstanter Leistung $P$ angelegt werden kann. Aus dem Hohlraum werden die elektrischen Anschlussleitungen durch Kanäle spannweitig aus dem Modell geführt, wo sich die Stromquelle befindet.

Die Breite der Heizelemente beträgt $0.4 \mathrm{~mm}$. Um den Einfluss der Heizelementlänge zu untersuchen, beträgt diese in vier der acht Heizreihen $10 \mathrm{~mm}$, und in den restlichen vier Heizreihen beträgt sie $5 \mathrm{~mm}$. Die Reihen mit langen und kurzen Heizelementen sind in Profiltiefenrichtung abwechselnd angeordnet. Durch die zwei vorgesehenen unterschiedlichen Längen der Heizelemente ergibt sich für die jeweiligen Heizreihen zwangsweise eine leicht verschiedene Geometrie der Leitelemente. Daher sind in Abbildung 4.5.3 
(a) Heizreihen 1,3,5 und 7

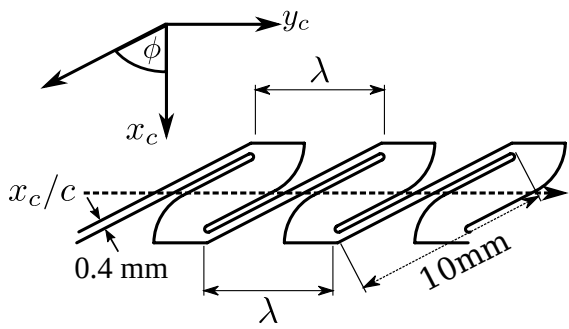

(b) Heizreihen 2,4,6 und 8

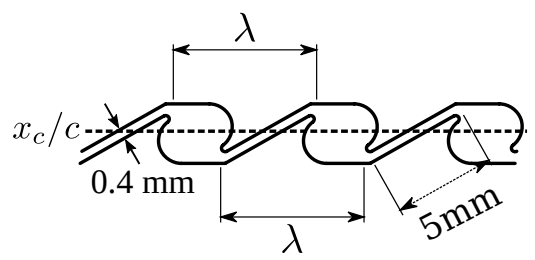

Abbildung 4.5.3: Die beiden unterschiedlichen verwendeten Heizreihen-Geometrien (die mittlere Plattentiefenposition ist als gestrichelte Linie markiert)

\begin{tabular}{cccc} 
Heizreihen-Nr. & $x_{c} / c$ & Länge $[\mathrm{mm}]$ & $\phi\left[^{\circ}\right]$ \\
\hline 1 & 0.050 & 10 & -62.71 \\
2 & 0.058 & 5 & -60.50 \\
3 & 0.066 & 10 & -59.35 \\
4 & 0.074 & 5 & -57.82 \\
5 & 0.083 & 10 & -56.98 \\
6 & 0.091 & 5 & -55.81 \\
7 & 0.100 & 10 & -55.17 \\
8 & 0.109 & 5 & -54.26
\end{tabular}

Tabelle 4.5.1: Mittlere Profiltiefenpositionen, Länge und Winkel der Heizelemente der acht Heizreihen des Heizaktuators

die beiden Geometrien exemplarisch im Vergleich dargestellt. Die mittleren Profiltiefenpositionen der acht Heizreihen sind in Tabelle 4.5.1 angegeben. Die Position der Heizreihe 1 befindet sich damit besonders nah am Neutralpunkt der ausgewählten Kontrollmode. Die hier eingeführte Nummerierung der Heizreihen wird bei der Diskussion der Ergebnisse verwendet werden.

Die Kupferelemente stellen bereits im unbeheizten Zustand eine signifikante geometrische Rauigkeit dar. Im Profiltiefenbereich der Heizreihen ist die Grenzschicht zudem sehr rezeptiv für initiale Störungen. Es gilt soweit wie möglich zu verhindern, dass zusätzliche stationäre QSI durch die geometrische Rauigkeit angeregt werden. Dafür wird eine Abdeckschicht aus einem Füllmaterial auf die fixierte Abdeckfolie aufgetragen. Um dabei eine einheitliche Höhe zu definieren, sind in Bereichen, in denen weder Heiz- noch Leitelemente benötigt werden, ebenfalls Kupferelemente angeordnet. Zwischen ihnen sind Zwischenräume freigestellt, in die das Füllmaterial eindringt und somit die 
hohe Wärmeleitfähigkeit der Kupferelemente regelmäßig durch seine niedrigere Wärmeleitfähigkeit unterbricht. Um eine möglichst hohe spannweitige Periodizität der Wandtemperatur erzeugen zu können, sollte die spannweitige Wärmeleitfähigkeit so gering wie möglich sein.

Als Füllmaterial wurde in ersten Experimenten ein nach Herstellerangaben „hochtemperaturfester" Acryllack verwendet, welcher üblicherweise zur Lackierung von Öfen verwendet wird. In ersten Windkanalexperimenten wurde aber festgestellt, dass der Betrieb der Heizreihen die Oberflächenbeschaffenheit selbst veränderte. Nach dem Betrieb wurde bei Wiederholungsmessungen in der Grenzschicht stromab mit ausgeschaltetem Aktuator eine veränderte stationäre Störströmung beobachtet. Die Anforderungen an die Reproduzierbarkeit der Oberflächenbeschaffenheit als relevante Anregung stationärer QSI liegen dabei im Mikrometerbereich.

Für die im Rahmen dieser Arbeit beschriebenen Untersuchungen kam daher als Füllmaterial ein Epoxidharz namens „MP Advanced“ des Herstellers „R\&G Faserverbundwerkstoffe“ zum Einsatz, welches nach dem Aushärten einen Duroplasten darstellt und sich in Vorversuchen als äußerst temperaturstabil erwiesen hatte. Zum Aushärten des Epoxidharzes wurde der gesamte Aktuatoreinsatz in einem Industrieofen erhitzt. Dabei wurde die Temperatur zunächst innerhalb von 30 Minuten auf $373 \mathrm{~K}$ angehoben. Diese Temperatur wurde für sieben Stunden konstant gehalten, um sie dann innerhalb von vier Stunden auf $413 \mathrm{~K}$ zu erhöhen. Diese Temperatur wurde wiederum 10 Stunden konstant gehalten, bevor die Ofentemperatur innerhalb von drei Stunden auf Raumtemperatur gesenkt wurde. Nach dem Aushärten wurde das Material manuell geschliffen, um die Amplitude der Periodizität der Modelloberflächengeometrie im für Anregung von stationären QSI relevanten Wellenzahlbereich zu reduzieren. Dabei blieben die Kupferelemente aber von einer ununterbrochenen Epoxidschicht bedeckt. Der gemittelte Mittenrauwert dieser Oberfläche betrug final $\bar{R}_{a}=2.5(9) \mu \mathrm{m}$. Die Oberfläche wird in Abschnitt 7.1 detailliert charakterisiert.

\subsubsection{Rotationsaktuator}

Wie in Abschnitt 2.5.3 beschrieben, sollte eine spannweitig periodische Anordnung rotierender Scheiben in der Lage sein, eine geeignete Störung in die Grenzschichtströmung einzubringen, um eine stationäre QSI-Mode anzuregen. Die spannweitige Wellenlänge $\lambda=6 \mathrm{~mm}$ der ausgewählten Kontrollmode schränkt dabei den zur Verfügung stehenden Bauraum für die nötigen mecha- 
(a)

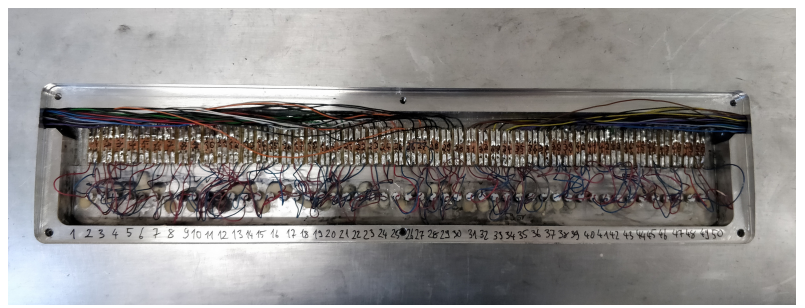

(b)

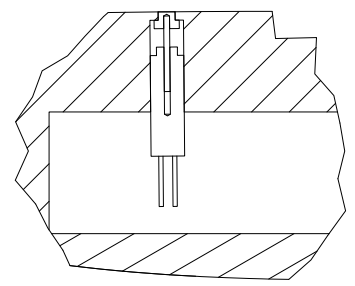

Abbildung 4.5.4: (a) Foto des geöffneten Hohlraums auf der Unterseite des Rotationsaktuator-Naseneinsatzes mit eingebauten Motoren und elektrischen Zuleitungen (b) Ausschnitt des Querschnitts des Rotationsaktuator-Naseneinsatzes mit einer einzelnen rotierenden Scheibe, welche durch einen Miniatur-Elektromotor angetrieben wird.

nischen Elemente eines solchen Aktuators ein. Die im Rahmen dieser Arbeit realisierte technische Lösung ist in Abbildung 4.5.4(b) als Querschnittsskizze dargestellt.

Es wurde ein spezieller Naseneinsatz aus einer Aluminium-Legierung gefertigt, in dem an der Plattentiefenposition $x_{c} / c=0.1$ auf der Modelloberseite 50 spannweitig periodisch verteilte Bohrungen mit einem Abstand von $\lambda=6 \mathrm{~mm}$ zueinander angeordnet sind (Abb. 4.5.5). Diese Bohrungen besitzen einen Durchmesser von $2.10 \mathrm{~mm}$, in dem die rotierenden Scheiben mit einem leicht geringerem Durchmesser von $d=2.0 \mathrm{~mm}$ Platz finden. Unter jeder Bohrung befindet sich ein Sackloch mit einem Durchmesser von $4.2 \mathrm{~mm}$, welches in einen Hohlraum mündet, der auf der Modellunterseite mit einem Deckel luftdicht verschlossen werden kann.

Die rotierenden Scheiben sind als sog. Drehteller realisiert. Jeder Drehteller ist aus einer Aluminium-Legierung gefertigt und besitzt auf der zur Strömung exponierten Seite einen Durchmesser von $d=2.0 \mathrm{~mm}$ und auf der unteren Seite einen Durchmesser von $3 \mathrm{~mm}$, wodurch ein Kragen entsteht. Der Drehteller wird mithilfe eines Miniatur-Elektromotors in Rotation versetzt, an dessen Motorachse der Drehteller fixiert ist. Zum Einbau der Motoren wird der Naseneinsatz mit seiner Unterseite nach oben orientiert, sodass der Hohlraum von oben zugänglich ist. Der geöffnete Hohlraum mit eingebauten Motoren und verbundenen elektrischen Anschlüssen ist als Fotografie in Abbildung 4.5.4(a) dargestellt. Der Elektromotor und der an der Achse fixierte Drehteller werden im Hohlraum in das Sackloch gesetzt, während der Motor in Betrieb 


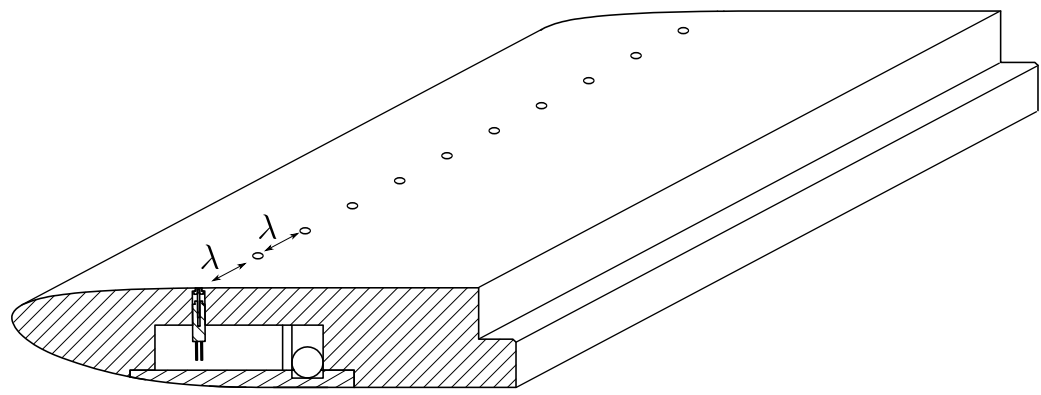

Abbildung 4.5.5: Dreidimensionale Skizze eines spannweitigen Ausschnitts des aufgeschnittenen Rotationsaktuator-Naseneinsatzes mit rotierenden Scheiben

ist und der Drehteller rotiert. Der Drehteller wird dabei so in die Durchgangsbohrung zur Oberseite des Naseneinsatzes geführt, dass der Teil geringeren Durchmessers frei rotiert und seine Oberseite bündig mit der Oberseite des Einsatzes abschließt. In dieser Position schleift der Kragen des Drehtellers auf der Unterseite der Sacklochdecke, wobei Graphitpulver als Schmiermittel dient. Das Eigengewicht des Motors drückt dabei den Drehteller an. Bei rotierendem Drehteller wird ein Klebstoff zwischen dem Gehäuse des Motors und der Wand des Sackloches appliziert, der den Motor in seiner Position fixiert. Damit wird erreicht, dass der trocknende Klebstoff den Motor in einer Position fixiert, in der die freie Rotation des Drehtellers möglich bleibt. Um eine spektral möglichst reine Anregung der stationären Kontrollmode mit einer spannweitigen Wellenlänge von $\lambda=6 \mathrm{~mm}$ zu erreichen, wurden 50 der beschriebenen Anordnungen in einer spannweitigen Reihe mit diesem periodischen Abstand eingesetzt.

Eine schematische Darstellung eines Ausschnitts des Naseneinsatzes ist zur Verdeutlichung dieser Anordnung in Abbildung 4.5.5 dargestellt. In dem Hohlraum unterhalb der Motoren sind die elektrischen Anschlussleitungen der Motoren verlegt, welche durch seitliche Kanäle spannweitig aus dem Modell geführt werden. Der Hohlraum wird an der Unterseite des Einsatzes durch einen konturierten Deckel verschlossen. Um zu vermeiden, dass durch die oben beschriebenen Spaltmaße und durch den Hohlraum ein Druckausgleich zwischen der Oberseite und der Unterseite der Modellplatte stattfindet, wird der Hohlraum mithilfe von Vakuumfett am Deckel abgedichtet. Die seitlichen Kanäle mit den elektrischen Zuleitungen werden mit flüssigem Kerzenwachs abgedichtet. 


\section{Regelung der Rotationsfrequenz}

Um stationäre QSI mit einer reproduzierbaren Amplitude anzuregen, müssen die Elektromotoren mit einer zeitlich konstanten, reproduzierbaren und bekannten Rotationsfrequenz $f_{\text {rot }}$ betrieben werden. In ersten Tests wurde festgestellt, dass die Rotationsfrequenz der Motoren mit fixierten Drehtellern im eingebauten Zustand nicht wie im freilaufenden Zustand in einem einfachen linearen Zusammenhang zur Betriebsspannung des Motors stand. Bei gleicher Betriebsspannung stellte sich bei unterschiedlichen Drehtellern eine verschiedene Rotationsfrequenz ein, welche zudem nicht reproduzierbar war. Denkbare relevante Einflüsse auf dieses Verhalten sind die unterschiedliche Einbaulage der Drehteller, Effekte von thermischer Ausdehnung oder ein Einfluss vom Abrieb der Drehtellerkragen. Daher musste jeder der 50 Motoren einzeln in seiner Rotationsfrequenz geregelt werden. Dafür war keine kommerzielle Lösung verfügbar, weshalb eigens eine Regelung entwickelt wurde.

Die verwendeten Elektromotoren sind Gleichstrommotoren mit einem DreiSegment-Kommutator und Schleifkontakten. Pro Umdrehung wird der elektrische Stromfluss also drei Mal unterbrochen. Es wird die an einem Vorwiderstand vor jedem einzelnen Motor abfallende Spannung zeitlich hochaufgelöst mit einem Datenerfassungsgerät (Analog-Digital-Wandler) erfasst und aus der Periodizität des Signals mittels Auswertung des Frequenzspektrums die Rotationsfrequenz $f_{\text {rot }}$ bestimmt. Die korrekte Erkennung der Rotationsfrequenz wurde vor den Windkanalexperimenten mithilfe eines Stroboskops mit einstellbarer Blitzfrequenz und Markierungen auf den rotierenden Drehtellern verifiziert. Die so erkannte Rotationsfrequenz wird in einer in LabView geschriebenen Software einem digitalen Proportional-Integral-Derivativ-Regelalgorithmus als Eingangsgröße zugeführt, welcher sie mit einem Sollwert vergleicht und eine Stellgröße regelt. Als Stellgröße dient die Betriebsspannung des Motors, welche ohne Last bzw. im nicht eingebauten Zustand in einem linearen Zusammenhang mit der Rotationsfrequenz steht. Da in den verwendeten Spannungsausgabegeräten (Digital-Analog-Wandler) nur ein sehr geringer Strom fließen darf, ist für jeden Motor eine TransistorEmitterschaltung nötig, welche den geringen Steuerstrom auf den nötigen Betriebsstrom des Motors verstärkt. Die so einzeln regelbaren Motoren erlauben es, zusätzlich zur Anregung einer Störung mit der spannweitigen Auslegungswellenlänge $\lambda=6 \mathrm{~mm}$ eine Anregung mit einem ganzzahligen Vielfachen dieser Wellenlänge oder eine Anregung durch einen einzeln betriebenen Drehteller zu untersuchen. Der beschriebene Regelkreislauf ist schematisch 


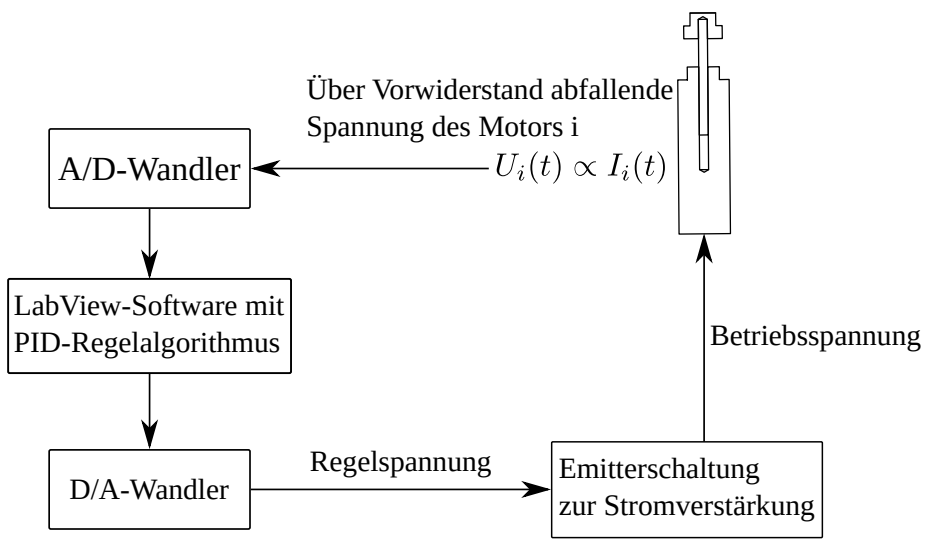

Abbildung 4.5.6: Schematische Darstellung der individuellen Regelung der Motoren bzgl. ihrer Rotationsfrequenz

in Abbildung 4.5.6 dargestellt. Die so geregelte Rotationsfrequenz wurde im Bereich $166.67 \mathrm{~Hz} \leq f_{\text {rot }} \leq 1083.33 \mathrm{~Hz}$ variiert. Leider ergaben sich durch die einfache Lagerung der Drehteller trotz der Regelung teilweise Einschränkungen bzgl. der konstanten Rotationsfrequenz mit dem Sollwert. Daher musste bei den Untersuchungen der spannweitige Messbereich auf den Nachlauf der längsten ununterbrochenen spannweitigen Reihe von einwandfrei rotierenden Drehtellern eingeschränkt werden, siehe Abschnitt 8.1.1. 


\section{Kapitel 5}

\section{Experimentelle Methoden und Versuchstechnik}

In diesem Kapitel werden die im Rahmen der Arbeit verwendeten experimentellen Methoden, Abläufe und Versuchstechniken beschrieben und mögliche Fehler diskutiert. Des Weiteren werden im Folgenden Ergebnisse entsprechender Voruntersuchungen zu deren Charakterisierung präsentiert.

\subsection{Hitzdrahtmessungen}

Zur Untersuchung der Vorgänge in der Grenzschicht kam KonstanttemperaturHitzdrahtanemometrie zum Einsatz. Dabei wird ein sehr dünner Draht, der Hitzdraht, durch einen elektrischen Heizstrom auf eine Temperatur deutlich über der Fluidtemperatur geheizt. Durch die Strömung wird konvektiv Wärme vom Hitzdraht abgeführt. Mittels einer elektronischen Regelung wird unter Ausnutzung der Temperaturabhängigkeit des elektrischen Widerstandes die Drahttemperatur konstant gehalten. Die dabei zugeführte elektrische Energie entspricht somit der konvektiv abgeführten Wärmemenge, die wiederum maßgeblich von Betrag und Richtung der Anströmgeschwindigkeit sowie von der Fluidtemperatur abhängt. Dieser Zusammenhang kann durch eine physikalische Modellierung hergestellt werden, siehe [16, 78, 81]. In der Praxis ist jedoch immer eine Kalibrierung notwendig, da viele Einflussgrößen, u.a. 


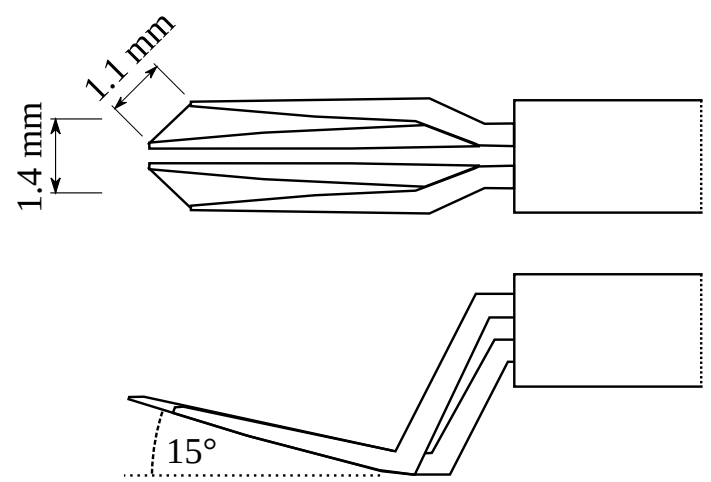

Abbildung 5.1.1: Skizze der eingesetzten V-Draht-Sonden in Aufsicht (oben) und Seitenansicht (unten), nach Produktabbildung des Herstellers DANTEC Dynamics

die exakte Geometrie der verwendeten Sonde, theoretisch nur unzureichend erfasst und berücksichtigt werden können. Im folgenden Abschnitt 5.1.1 werden zwei unterschiedliche Kalibrierverfahren näher erläutert.

Es kamen Anemometer vom Typ DISA 55M01 und spezielle Grenzschicht-VDraht-Sonden der Firma DANTEC Dynamics zum Einsatz. An der gekröpften Sondenspitze sind zwei mit Gold beschichtete Hitzdrähte aus Wolfram mit einem Durchmesser von $5 \mu \mathrm{m}$ in einem Winkel von $90^{\circ}$ zueinander in einer Ebene angebracht, siehe Abbildung 5.1.1. Durch den parallelen Betrieb zweier Anemometer an jeweils einem Draht lassen sich so zwei Geschwindigkeitskomponenten in der gemeinsamen Drahtebene messen. Dabei wird im Folgenden die Annahme getroffen, dass die wandnormale Geschwindigkeitskomponente $w$ in der Grenzschicht vernachlässigbar klein ist und die konvektive Kühlung der Drähte durch die wandparallelen Komponenten dominiert wird. Die Drähte hatten jeweils eine Länge von $1.1 \mathrm{~mm}$ und einen seitlichen Abstand von Drahtmitte zu Drahtmitte von $1.4 \mathrm{~mm}$. Der Kröpfungswinkel betrug $15^{\circ}$ und die Sonde wurde so gehalten, dass sich die durch die Drähte aufgespannte Ebene parallel zur Modelloberfläche befand.

Die V-Draht-Sonde wurde am Ende eines Haltearms befestigt, welcher im rechten Winkel in einer vertikalen Vierkantsäule eingespannt wurde. Diese schloss am oberen Ende an die Säule einer Traversiereinrichtung an, die wiederum oberhalb der offenen Messstrecke angebracht war. Dieser Aufbau ist in Abb. 5.1.2 dargestellt. Er ermöglichte es, die Sonde von der stromab gelegenen Seite zwischen Modellplatte und Verdrängungskörper zu traversieren. Im Haltearm waren in regelmäßigen Abständen von 100 mm Durchgangs- 


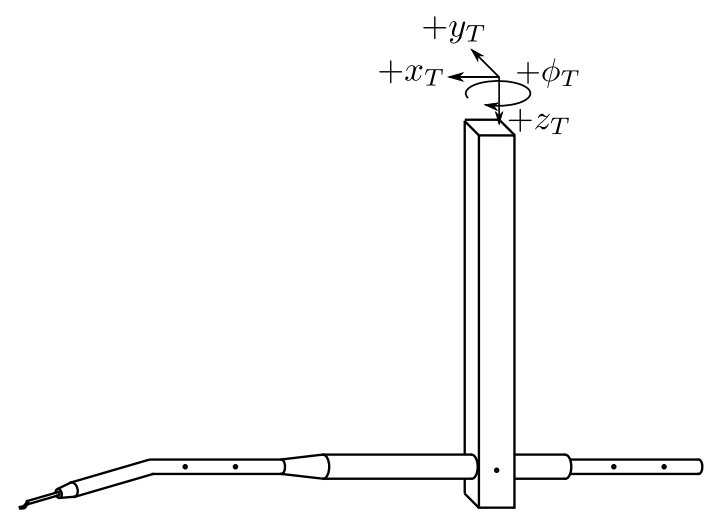

Abbildung 5.1.2: Skizze des Haltearms für Hitzdrahtmessungen und Anschluss an die Traversiereinrichtung

bohrungen vorgesehen, sodass der Abstand der Sonde von der vertikalen Vierkantsäule verändert werden konnte. Dies ermöglichte Hitzdrahtmessungen an spannweitig verteilten Positionen entlang der gesamten Plattentiefe sowie Messungen stromauf und stromab der Modellplatte.

Die drei translatorischen Achsen der Traversiereinrichtung sind mit $x_{T}, y_{T}$, $z_{T}$ bezeichnet. Die Traversiereinrichtung ermöglicht auch eine Rotation um die $z_{T}$-Achse mit dem Winkel $\phi_{T}$, was vor allem zur Kalibrierung der Sonden genutzt wurde, siehe Abschnitt 5.1.3. Die durch den Schrittmotorantrieb bedingten Schrittweiten betragen in den vier Bewegungsrichtungen:

$$
\begin{aligned}
\delta x_{T} & =12.5 \mu \mathrm{m} \\
\delta y_{T} & =12.5 \mu \mathrm{m} \\
\delta z_{T} & =7.5 \mu \mathrm{m} \\
\delta \phi_{T} & =\frac{1}{400}^{\circ}
\end{aligned}
$$

Die Traversiereinrichtung, der Haltearm und natürlich auch die Hitzdrahtsonde sind mechanischen Vibrationen ausgesetzt. Mithilfe eines Beschleunigungssensors konnten diese Vibrationen an mehreren Positionen entlang der Traversiereinrichtung und der Stahlträgerkonstruktion oberhalb der Messstrecke festgestellt werden.

Sondenvibrationen stellen einen unerwünschten Einfluss auf Hitzdrahtmessungen dar, da einerseits die Schwingungsbewegung der Hitzdrahtsonde zu einer induzierten Geschwindigkeitskomponente führt, welche die Drähte insta- 


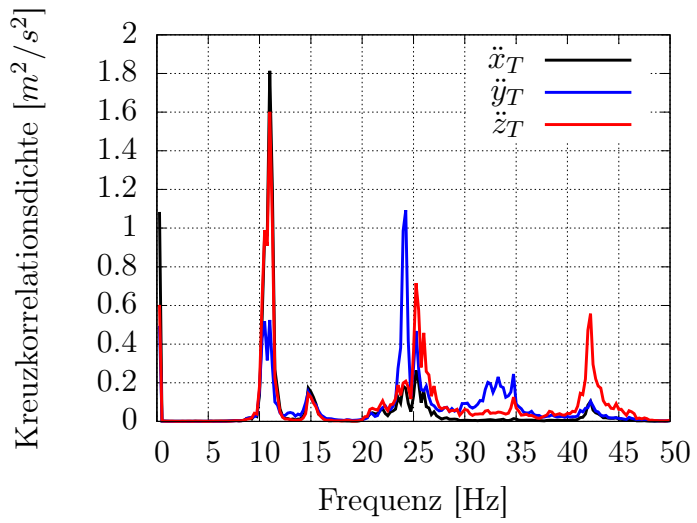

Abbildung 5.1.3: Exemplarisches Kreuzkorrelationsspektrum der gemessenen mechanischen Beschleunigung und der gemessenen Geschwindigkeitsschwankung in der Grenzschicht an einer ausgewählten Position

tionär zusätzlich zur Anströmgeschwindigkeit kühlt. Des Weiteren führt die Auslenkung der Sonde in der Grenzschicht mit starken örtlichen Geschwindigkeitsgradienten dazu, dass an einer nominellen Messposition scheinbar große Geschwindigkeitsschwankungen gemessen werden. Diese Geschwindigkeitsschwankungen existieren aber nur im bewegten Bezugssystem der Sonde und dürfen nicht als instationäre Geschwindigkeitsschwankungen in der Grenzschicht interpretiert werden.

Um diese Effekte bei der Interpretation der Ergebnisse von den aerodynamischen Effekten der Grenzschichtströmung trennen zu können, wurde ein 3-Achsen-Beschleunigungssensor des Typs „Analog Devices ADXL330“ am Haltearm nahe der Hitzdrahtsonde befestigt und sein Signal während aller Hitzdrahtmessungen erfasst. Berechnet man aus den mit gleicher Abtastrate aufgenommenen Signalen des Beschleunigungssensors und der Hitzdrahtsonde das Kreuzkorrelationsspektrum, ergeben sich lokale Maxima hoher Korrelation. Bei diesen Frequenzen findet am wahrscheinlichsten ein signifikanter störender Einfluss der mechanischen Vibrationen auf Hitzdrahtmessungen statt. Ein Beispiel eines solchen Kreuzkorrelationsspektrums ist in Abbildung 5.1.3 dargestellt. Zur Berechnung des Spektrums wurde die Funktion „csd“ der Software-Bibliothek Matplotlib [47] verwendet. Dabei sind diskrete lokale Maxima in $x_{T}, y_{T}$ und $z_{T}$-Richtung zu erkennen, deren Überlagerung einer nicht-trivialen Bewegung der Sonde entspricht. Solche lokalen Maxima hoher Korrelation können bei der Interpretation instationärer Hitzdrahtsignale berücksichtigt werden. Insbesondere wurden vor der Berechnung von 


\begin{tabular}{l|c|c|c} 
& $x_{T}$-Richtung & $y_{T}$-Richtung & $z_{T}$-Richtung \\
\hline$s\left(\ddot{x}_{T}\right), s\left(\ddot{y}_{T}\right), s\left(\ddot{z}_{T}\right)\left[\mathrm{m} / \mathrm{s}^{2}\right]$ & 0.22 & 1.05 & 0.45 \\
$s\left(\dot{x}_{T}\right), s\left(\dot{y}_{T}\right), s\left(\dot{z}_{T}\right)[\mathrm{m} / \mathrm{s}]$ & $2 \cdot 10^{-3}$ & $7 \cdot 10^{-3}$ & $2 \cdot 10^{-3}$ \\
$s\left(x_{T}\right), s\left(y_{T}\right), s\left(z_{T}\right)[\mu \mathrm{m}]$ & 29 & 54 & 24
\end{tabular}

Tabelle 5.1.1: Exemplarische Standardabweichungen der instationären Werte für Beschleunigung, Geschwindigkeit und Auslenkung des Beschleunigungssensors

Effektivwerten instationärer Geschwindigkeitsfluktuationen jene spektralen Anteile, die signifikant mit einem der drei Signale des Beschleunigungssensors korrelierten, entfernt. Dies betraf ausschließlich spektrale Anteile im Frequenzbereich $f<100 \mathrm{~Hz}$. Das Entfernen solcher Signalanteile verhindert, dass mechanische Vibrationen in Effektivwerten als instationäre Geschwindigkeitsfluktuationen fehlinterpretiert werden. Es bedeutet aber auch, dass echte instationäre Geschwindigkeitsfluktuationen bei diesen Frequenzen in Effektivwerten nicht berücksichtigt werden. Bei der Darstellung von Spektren instationärer Geschwindigkeitsfluktuationen werden keine Anteile entfernt.

Durch Integration der zeitlich aufgelösten Signale des Beschleunigungssensors erhält man die Geschwindigkeit und Auslenkung des Sensors. Dabei werden die Signale vor jeder Integration mit einem Hochpass der Grenzfrequenz $f_{G}=5 \mathrm{~Hz}$ gefiltert, um eine Drift zu vermeiden. Dies wurde anhand einer für Grenzschichtuntersuchungen repräsentativen Messung (die Hitzdrahtsonde befand sich bei $x_{c} / c=0.5$ in der Grenzschicht) während einer Gesamtdauer von $300 \mathrm{~s}$ an einer einzelnen Position durchgeführt. Aus den ermittelten instationären Beschleunigungen, Geschwindigkeiten und Auslenkungen in $x_{T}$, $y_{T}$ und $z_{T}$-Richtung wurde jeweils die Standardabweichung $s$ gebildet und diese in Tabelle 5.1.1 aufgeführt.

Durch die starre Verbindung der Hitzdrahtsonde mit dem Haltearm und dem Beschleunigungssensor vibriert die Sonde mit derselben Geschwindigkeit wie der Sensor. Der Effektivwert der erwarteten instationären induzierten Geschwindigkeitsschwankung setzt sich abhängig von der Richtungsabhängigkeit der Sonde aus den Schwankungsgrößen $s\left(\dot{x}_{T}\right), s\left(\dot{y}_{T}\right)$ und $s\left(\dot{z}_{T}\right)$ des Sensors additiv zusammen. Er wird in Summe aber in ähnlich geringer Größenordnung wie die dargestellten Werte erwartet. Neben den Einschränkungen bzgl. der Interpretation instationärer Geschwindigkeitsinformationen der Hitzdrahtsonde schränken die Auslenkungen auch die minimal vermessbaren Wandabstände ein, da die Sonde sonst auf der Modelloberfläche aufschlägt. Da die Sonde etwas weiter vom Aufhängungspunkt der Traversiereinrichtung entfernt fixiert ist als der Beschleunigungssensor und größere Auslenkungen auftreten 
als die Standardabweichung, ist der minimal vermessbare Wandabstand auf erfahrungsgemäß etwa $z=60 \mu \mathrm{m}$ eingeschränkt.

\subsubsection{Kalibrierung und Auswertung}

Mithilfe zweier Hitzdraht-Anemometer werden als Maß für die elektrische Heizleistung zwei Brückenspannungen $E_{1}$ und $E_{2}$ als Messsignale erfasst. Unter der Annahme von vernachlässigbarem Wärmetransport senkrecht zur gemeinsamen Drahtebene enthalten diese Spannungen die Information über den Geschwindigkeitsbetrag $U$ der Anströmung und ihren Anströmwinkel $\phi_{S o}$ in dieser Ebene. Um diese Information aus gemessenen Wertepaaren $\left(E_{1}, E_{2}\right)$ auswerten zu können, ist eine eindeutige Abbildung $\left(E_{1}, E_{2}\right) \rightarrow\left(U, \phi_{S o}\right)$ nötig. Zum Herstellen dieser Abbildung wurden zwei unterschiedliche Verfahren implementiert und verglichen, welche in den nächsten beiden Abschnitten beschrieben werden.

\section{Getrennte Kalibrierung von Geschwindigkeits- und Richtungsabhängigkeit}

In früheren Arbeiten am DLR Göttingen, bspw. [93] und [76], wird eine getrennte Kalibrierung von Geschwindigkeits- und Richtungsabhängigkeit der Spannungen basierend auf physikalischen Modellen des konvektiven Wärmeabtransports verwendet. Die gesuchte Abbildung zwischen AnemometerBrückenspannungen und Geschwindigkeiten wird im Wesentlichen durch Geschwindigkeitskoeffizienten $c_{i}$ und Richtungskoeffizienten $a_{i}$ in Modellgleichungen hergestellt, wie im Folgenden skizziert wird. In diesem Abschnitt wird die Herleitung dieser Abbildung aus [93] speziell für den in dieser Arbeit relevanten Fall der V-Draht-Sonden wiedergegeben, wobei lediglich die Geschwindigkeitskomponenten in der gemeinsamen Drahtebene berücksichtigt werden. Für die Bestimmung der Geschwindigkeitskoeffizienten lässt sich für jeden der beiden Drähte ein polynomieller Ansatz wie folgt aufstellen:

$$
U_{k}^{*}=\sum_{i=0}^{4} c_{i} \cdot E^{* i}
$$

Dabei ist $U_{k}$ die normierte effektive Kühlgeschwindigkeit des Drahtes, welche bei Anströmung entlang der Sondenachse der kalibrierten Anströmgeschwindigkeit $U$ entspricht. Die Normierung berücksichtigt den Winkel der Drähte zur Sondenachse und wird hier nicht weiter thematisiert. Sie wird von Rose- 
mann im Detail beschrieben [93]. Dort trägt diese normierte Kühlgeschwindigkeit den Index $e$, welches hier zwecks Konsistenz vermieden wurde. Der Zusatz „*" bezeichnet eine Kompensation des Einflusses der Fluidtemperatur, welche wie folgt definiert ist:

$$
\begin{aligned}
E^{*} & =\left(\frac{T_{f}}{T_{f_{0}}}\right)^{-0.38} \cdot\left(\frac{T_{W}-T_{0}}{T_{W}-T}\right)^{0.5} \cdot E \\
U_{k}^{*} & =\frac{p}{p_{0}} \cdot\left(\frac{T_{f}}{T_{f_{0}}}\right)^{-1.76} \cdot U_{k}
\end{aligned}
$$

Der Index 0 beschreibt einen bekannten Referenzzustand. Die sog. „Filmtemperatur" $T_{f}$ entspricht dem Mittelwert aus Drahttemperatur $T_{W}$ und Fluidtemperatur $T$ :

$$
T_{f}=\frac{T_{w}+T}{2}
$$

Dabei muss die Fluidtemperatur $T$ unabhängig gemessen werden. Die Drahttemperatur $T_{W}$ ergibt sich aus dem sog. „Überhitzungsverhältnis“ $\chi$, welches am Anemometer eingestellt wird. Es beschreibt, wie stark der Widerstand des Drahtes im geheizten Zustand und damit die Drahttemperatur im Vergleich zum Referenzzustand erhöht wird (mit dem materialspezifischen Temperaturkoeffizienten $\xi$ ):

$$
\begin{aligned}
\chi & =\frac{R_{W}-R_{W, 0}}{R_{W, 0}} \\
R_{W} & =R_{W, 0}\left(1+\xi\left(T_{W}-T_{W, 0}\right)\right) \\
T_{W} & =T_{W, 0}+\frac{\chi}{\xi}
\end{aligned}
$$

Die vier Koeffizienten $c_{i}$ können für jeden Draht bestimmt werden, indem die Sonde bei einem konstanten Sondenschiebewinkel von $\phi_{S o}=0^{\circ}$ mehreren bekannten Anströmgeschwindigkeiten ausgesetzt wird. Mit einem Anpassungsalgorithmus basierend auf der Minimierung der Summe der Fehlerquadrate wird dann das Polynom 5.1.5 an die gemessenen Spannungen $E^{*}$ und die Werte von $U_{k}^{*}$ angepasst.

Für die Richtungsabhängigkeit der Sonde wird zunächst für beide Drähte unabhängig folgender Ansatz gemacht:

$$
U_{k}^{2}=a_{1} u^{2}+2 a_{2} u v+a_{3} v^{2}
$$

Bei einer konstanten und bekannten Anströmgeschwindigkeit $U$ wird diese anhand des variierten Winkels $\phi_{S o}$ in Komponenten $u$ und $v$ zerlegt. Für den 
jeweiligen Winkel wird mithilfe der Geschwindigkeitskalibrierung und der gemessenen Spannungen die Kühlgeschwindigkeit $U_{k}$ bestimmt. Nun lassen sich die die drei Richtungskoeffizienten $a_{i j} \quad(j=1,2,3)$ für den jeweiligen Draht $i$ mit einem Anpassungsalgorithmus basierend auf der Minimierung der Summe der Fehlerquadrate gemäß Gleichung 5.1.12 bestimmen.

Misst man mit der kalibrierten Sonde nun Brückenspannungen $E_{1}$ und $E_{2}$, kann man mithilfe der kalibrierten Geschwindigkeitskoeffizienten und Gleichungen 5.1.5, 5.1.6 und 5.1.7 normierte Kühlgeschwindigkeiten $U_{k 1}$ und $U_{k 2}$ für die beiden Drähte errechnen. Mit den nun gesuchten Komponenten $u_{S o}=U \cos \phi_{S o}$ und $v_{S o}=U \sin \phi_{S o}$ schreiben wir Gleichung 5.1.12 als:

$$
\begin{aligned}
& U_{k 1}{ }^{2}=a_{11} u_{S o}{ }^{2}+2 a_{12} u_{S o} v_{S o}+a_{13} v_{S o}{ }^{2} \\
& {U_{k 2}}^{2}=a_{21} u_{S o}{ }^{2}+2 a_{22} u_{S o} v_{S o}+a_{23} v_{S o}{ }^{2}
\end{aligned}
$$

und

$$
\begin{aligned}
& U_{k 1}{ }^{2}=U^{2} \cdot\left(a_{11} \cdot \cos ^{2} \phi_{S o}+2 a_{12} \cos \phi_{S o} \sin \phi_{S o}+a_{13} \sin ^{2} \phi_{S o}\right) \\
& U_{k 2}{ }^{2}=U^{2} \cdot\left(a_{21} \cdot \cos ^{2} \phi_{S o}+2 a_{22} \cos \phi_{S o} \sin \phi_{S o}+a_{23} \sin ^{2} \phi_{S o}\right)
\end{aligned}
$$

mit dem Geschwindigkeitsbetrag $U$ und dem Schiebewinkel $\phi_{S o}$ der Anströmung aus Sicht der Sonde. Durch Elimination von $U$ ergibt sich eine quadratische Gleichung für $\tan \phi_{S o}$ :

$$
d_{3} \tan ^{2} \phi_{S o}+2 d_{2} \tan \phi_{S o}+d_{1}=0
$$

mit

$$
d_{j}=a_{2 j}-\left(\frac{U_{k 2}}{U_{k 1}}\right)^{2} \cdot a_{1 j} \quad j=1,2,3 .
$$

Somit ergeben sich zwei Lösungen für $\phi_{S o}$, aus denen eine ausgewählt werden muss. Die Auswahl wird insbesondere anhand des Gültigkeitsbereichs der Sonde getroffen, mit dem gewisse Wertebereiche von $\phi_{S o}$ ausgeschlossen werden können, siehe [93]. Die beiden Lösungen lauten

$$
\phi_{S o}^{1,2}=\arctan \left(-\frac{d_{2}}{d_{3}} \pm \sqrt{\frac{d_{2}^{2}}{d_{3}{ }^{2}}-\frac{d_{1}}{d_{3}}}\right) .
$$

Der Geschwindigkeitsbetrag $U$ der Anströmung ergibt sich mit dem bekannten $\phi_{\text {So }}$ nun gleichermaßen aus Gleichung 5.1.15 oder 5.1.16. 
Der Vorteil dieser Kalibrier- und Auswertemethode besteht darin, dass die Geschwindigkeitsabhängigkeit nur bei einem einzelnen Anströmwinkel der Sonde und die Richtungsabhängigkeit nur bei einer einzelnen Anströmgeschwindigkeit kalibriert werden muss. Die Methode erfordert daher weniger zeitaufwendige Kalibriermessungen als die im Folgenden beschriebene Methode.

\section{Interpolationsmethode}

Eine weitere Möglichkeit, eine eindeutige Abbildung $\left(E_{1}, E_{2}\right) \rightarrow\left(U, \phi_{S o}\right)$ herzustellen, ist die Verwendung einer möglichst detaillierten Datenbank der beteiligten Größen. Dazu wird die V-Draht-Sonde systematisch mehreren bekannten Anströmgeschwindigkeiten und -winkeln in dem für Messungen relevanten Bereich ausgesetzt und die Brückenspannungen $E_{1}$ und $E_{2}$ gemessen. Aus der entstandenen Datenbank kann dann bei der Messung von Spannungen $E_{1}$ und $E_{2}$ die Anströmgeschwindigkeit und der Anströmwinkel zweidimensional interpoliert werden. Um den Aufwand zu reduzieren, wird für die Variation der Anströmgeschwindigkeit eine Verteilung mit vergleichsweise wenigen Stützstellen gewählt, zwischen denen interpoliert werden soll. Dafür wird für jeden kalibrierten Winkel an die kalibrierten Werte $E_{i}(U), i=1,2$ das sog. Kingsche Gesetz (Gleichung 5.1.20), welches aus physikalischen Überlegungen zum erzwungenen konvektiven Wärmeabtransport hergeleitet werden kann (siehe z.B. [78, 93]), angepasst. Diese Anpassung geschieht mithilfe eines Algorithmus zur Minimierung der Summe der Fehlerquadrate.

$$
E_{i}^{+^{2}}=A_{i}+B_{i} U^{n_{i}}
$$

Der hochgestellte Zusatz „+“" bezeichnet eine Temperaturkompensation der Spannung $E_{i}$, welche von derjenigen im vorigen Teilabschnitt abweicht. Dort wurde ein Einfluss der Fluidtemperatur sowohl in der Spannung als auch in der Kühlgeschwindigkeit des jeweiligen Drahtes kompensiert. Für jeden einzelnen der beiden Drähte wurde einer Spannung eine Kühlgeschwindigkeit zugeordnet. Die Temperaturkompensation für Spannung und Kühlgeschwindigkeit konnte für beide Drähte unabhängig erfolgen (Gleichungen 5.1.6 und 5.1.7). Bei der Interpolationsmethode hingegen wird der Einfluss der Fluidtemperatur nur anhand der jeweiligen Spannung kompensiert (siehe bspw. [78]), da dem korrigierten Spannungspaar direkt eine einzelne Anströmungsgeschwindigkeit (und eine Anströmungsrichtung) zugeordnet wird:

$$
E_{i}^{+}=\sqrt{E_{i}^{2} \frac{T_{W, i}-T_{0}}{T_{W, i}-T}}
$$




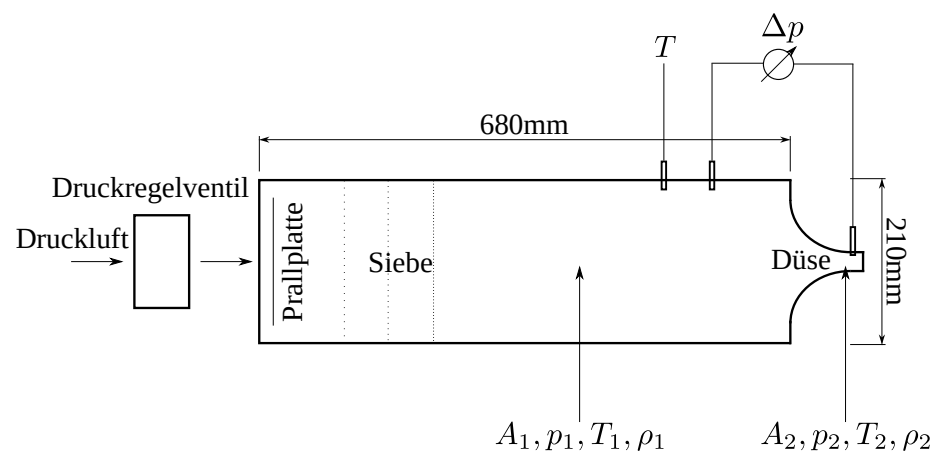

Abbildung 5.1.4: Querschnittsskizze des Hitzdrahtkalibrators

Dabei entspricht $T$ wieder der Fluidtemperatur während der Messung, $T_{W, i}$ der jeweiligen Drahttemperatur und $T_{0}$ der Fluidtemperatur während der Kalibrierung. Um bei der Auswertung physikalisch motiviert entlang der Anströmgeschwindigkeit interpolieren zu können, werden mithilfe von Gleichung (5.1.20) für jeden kalibrierten Winkel feiner verteilte Werte von $E_{i}(U), i=1,2$ berechnet als die tatsächlich kalibrierten Wertepaare. Aus der so entstandenen Datenbank von Wertepaaren $\left(E_{1}, E_{2}\right)$ und zugehörigen $\left(U, \phi_{S o}\right)$ wird dann bei einer Messung mit der kalibrierten Sonde zu gemessenen Spannungen der zugehörige Betrag von Anströmgeschwindigkeit und winkel mithilfe des 2D-Interpolationsalgorithmus griddata aus der SoftwareBibliothek SciPy [110] interpoliert, wobei eine kubische Polynomialinterpolation $[3,32]$ gewählt wurde.

Der Nachteil dieser Kalibrier- und Auswertungsmethode gegenüber der getrennten Kalibrierung von Geschwindigkeits- und Winkelabhängigkeit ist der deutlich höhere Zeitaufwand der Kalibrierung, da eine zweidimensionale Variation von $U$ und $\phi_{S o}$ durchgeführt werden muss. In Abschnitt 5.1.6 werden beide Methoden bzgl. des erwarteten methodischen Fehlers verglichen. Begründet durch das Ergebnis des Vergleichs wurde im Rahmen dieser Arbeit zur Auswertung der Hitzdrahtmessungen ausschließlich die Interpolationsmethode eingesetzt.

\subsubsection{Hitzdrahtkalibrator}

Um die V-Draht-Sonden bekannten Anströmgeschwindigkeiten aussetzen zu können, wurde ein Hitzdrahtkalibrator entworfen, der hauptsächlich aus einer Beruhigungskammer und einer Düse besteht und in Abbildung 5.1.4 als 
Querschnittsskizze dargestellt ist. Als Vorlage für den Entwurf diente dabei ein ähnlicher Kalibrator der TU Berlin. An die Beruhigungskammer wird über ein Druckregelventil Druckluft angeschlossen. Mithilfe des Druckregelventils lässt sich der Überdruck in der Beruhigungskammer relativ zum Umgebungsdruck und damit die Geschwindigkeit der ausströmenden Luft regeln.

In der Beruhigungskammer befindet sich hinter dem Druckluftanschluss eine Prallplatte zur Auffächerung der eintretenden Strömung. Stromab der Prallplatte befinden sich drei Turbulenzsiebe mit in Strömungsrichtung abnehmender Maschenweite. Die Distanz der Siebe zueinander wurde nach den Erkenntnissen von Groth et al. [39] gewählt, um sicherzustellen, dass die vom jeweiligen Sieb erzeugte Turbulenz zu kleineren Größenskalen zerfallen kann, bevor die Strömung auf das nächstfeinere Sieb trifft. In der Beruhigungskammer befindet sich auch ein PT100-Temperatursensor, um die Temperatur $T_{1}$ der strömenden Luft während der Kalibrierung messen zu können. Direkt stromab des Düsenaustritts wird die Hitzdrahtsonde während der Kalibrierung positioniert. Die Geschwindigkeit der ausströmenden Luft wird über den Differenzdruck $\Delta p=p_{1}-p_{2}$ zwischen dem statischen Druck $p_{1}$ in der Beruhigungskammer und im Düsenhals $p_{2}$ bestimmt. Zur Messung des Differenzdrucks sind jeweils Druckbohrungen eingelassen. Des Weiteren wird auch der Umgebungsdruck $p_{\infty}$ während der Kalibrierung gemessen. Die Kontur des Düsenzulaufs entspricht einem Kreissegment mit einem Radius von $60 \mathrm{~mm}$ und einem horizontalen Segment mit einer Länge von $20 \mathrm{~mm}$. Der Düseninnendurchmesser beträgt am Austritt $d_{2}=19.6 \mathrm{~mm}$. Die Geometrie des Kalibrators ist axialsymmetrisch um die Hauptströmungsachse und der Innendurchmesser der Beruhigungskammer beträgt $d_{1}=190 \mathrm{~mm}$. Somit gilt für das Kontraktionsverhältnis $\eta$ als Verhältnis der Düseneintritts- und Düsenaustrittsfläche $A_{1}$ und $A_{2}$ :

$$
\eta=\frac{A_{1}}{A_{2}}=\frac{\pi d_{1}^{2} / 4}{\pi d_{2}^{2} / 4}=\frac{d_{1}^{2}}{d_{2}^{2}} \approx 94
$$

Nimmt man den Zustand in der Beruhigungskammer mit den thermodynamischen Zustandsgrößen $p_{1}, T_{1}$ und $\rho_{1}$ als konstant an, und geht von einer adiabatischen Zustandsänderung bis zum Zustand im Düsenaustritt mit den Zustandsgrößen $p_{2}, T_{2}$ und $\rho_{2}$ aus, gilt:

$$
\frac{U_{2}^{2}}{2}-\frac{U_{1}^{2}}{2}=\frac{\kappa}{\kappa-1} R\left(T_{1}-T_{2}\right)
$$

Dies beschreibt die Enthalpieabnahme bei der Zunahme der kinetischen Energie, wobei $U_{1}$ der Strömungsgeschwindigkeit in der Beruhigungskammer 
und $U_{2}$ der Strömungsgeschwindigkeit im Düsenhals entspricht. Bei reversibel adiabatischer Zustandsänderung gilt

$$
\frac{T_{2}}{T_{1}}=\left(\frac{p_{2}}{p_{1}}\right)^{\frac{\kappa-1}{\kappa}} .
$$

Die relevante Temperatur für die Temperaturkompensation, wie sie bspw. in den Gleichungen 5.1.6 und 5.1.7 beschrieben wurde, ist also $T_{2}$, die erwartete Fluidtemperatur am Düsenaustritt. In Gleichung 5.1.23 lässt sich $T_{2}$ nun eliminieren. Des Weiteren liefert die Erhaltung des Massenstroms eine Beziehung zwischen $U_{1}$ und $U_{2}$ :

$$
\begin{aligned}
U_{1} \rho_{1} A_{1} & =U_{2} \rho_{2} A_{2} \\
U_{1} & =\frac{A_{2}}{A_{1}} \frac{\rho_{2}}{\rho_{1}} U_{2}
\end{aligned}
$$

Bei reversibler adiabatischer Zustandsänderung gilt weiterhin

$$
\frac{\rho_{2}}{\rho_{1}}=\left(\frac{p_{2}}{p_{1}}\right)^{\frac{1}{\kappa}} .
$$

Setzt man Gleichungen 5.1.24, 5.1.26 und 5.1.27 in Gleichung 5.1.23 ein, erhält man einen Ausdruck für die Strömungsgeschwindigkeit $U_{2}$ im Düsenaustritt:

$$
\begin{aligned}
U_{2}^{2}-\left(\frac{A_{2}}{A_{1}}\right)^{2}\left(\frac{p_{2}}{p_{1}}\right)^{\frac{2}{\kappa}} U_{2}^{2} & =\frac{2 \kappa}{\kappa-1} R T_{1}\left(1-\left(\frac{p_{2}}{p_{1}}\right)^{\frac{\kappa-1}{\kappa}}\right) \\
U_{2} & =\sqrt{\frac{\frac{2 \kappa}{\kappa-1} R T_{1}\left(1-\left(\frac{p_{2}}{p_{1}}\right)^{\frac{\kappa-1}{\kappa}}\right)}{1-\left(\frac{A_{2}}{A_{1}}\right)^{2}\left(\frac{p_{2}}{p_{1}}\right)^{\frac{2}{\kappa}}}}
\end{aligned}
$$

Zur Bestimmung der Austrittsgeschwindigkeit wird nun angenommen, dass am Düsenaustritt der statische Druck $p_{2}$ dem Umgebungsdruck $p_{\infty}$ entspricht und der statische Druck in der Beruhigungskammer entsprechend um den gemessenen Differenzdruck erhöht ist:

$$
\begin{aligned}
& p_{2}=p_{\infty} \\
& p_{1}=p_{\infty}+\Delta p
\end{aligned}
$$

Der Adiabatenexponent $\kappa=1.4$ und die spezifische Gaskonstante $R=$ $287.058 \mathrm{~J} /(\mathrm{kg} \cdot \mathrm{K})$ von Luft werden als konstant angenommen. Zusammen 
mit der gemessenen Temperatur in der Beruhigungskammer $T_{1}$ und dem bekannten Querschnittsverhältnis $\frac{A_{2}}{A_{1}}=\frac{1}{n}$ (siehe Gleichung 5.1.22) lässt sich nun die Strömungsgeschwindigkeit $U_{2}$ im Düsenhals bestimmen. Da die Sonde direkt stromab des Düsenaustritts positioniert wird, wird angenommen, dass diese Geschwindigkeit der für die Kühlung des Hitzdrahtes relevanten Strömungsgeschwindigkeit entspricht. Die Homogenität der erzeugten Geschwindigkeit stromab des Düsenaustritts wurde experimentell exemplarisch für eine feste Druckdifferenz $\Delta p$ untersucht. Dazu wurde eine kalibrierte Hitzdrahtsonde etwa an der Kalibrierposition rasterförmig entlang der Querschnittsfläche der Düse traversiert. Diese Messung zeigte eine gute Homogenität der Austrittsgeschwindigkeit. Eine quasi-dreidimensionale Simulation der Innenströmung des Hitzdrahtkalibrators mit TAU unter Annahme von Rotationssymmetrie zeigt zudem, dass die Strömung am Düsenaustritt keine signifikante Geschwindigkeitskomponente senkrecht zur Symmetrieachse aufweist. Des Weiteren zeigt sie, dass die Grenzschicht an der Düsenwand ausreichend dünn ist, dass sie den Geschwindigkeitsbetrag an der üblichen Kalibrierposition im Kern der Düsenströmung nicht wesentlich beeinflusst.

Um die Anwendbarkeit der Kalibrierung auf die Messung im Windkanal zu überprüfen, wurde eine Hitzdraht-V-Sonde vor dem Kalibrator kalibriert und mit ihr die Strömungsgeschwindigkeit im 1MG für unterschiedliche Kanalgeschwindigkeiten ausgewertet. In diesem Windkanal wurden die Grenzschichtuntersuchungen im Rahmen dieser Arbeit durchgeführt. Er wird in Abschnitt 5.2 näher beschrieben. Für die hier beschriebenen Voruntersuchungen war keine experimentelle Konfiguration in der Messstrecke eingebaut. Der ausgewertete Wert $U_{H D}$ wurde mit dem Ergebnis $U_{\text {Prandtl }}$ der Geschwindigkeitsmessung mithilfe einer Prandtl-Sonde verglichen. Dazu wurde die Hitzdrahtsonde in der leeren offenen Messstrecke des Ein-Meter-Kanals horizontal und vertikal mittig in einem Abstand entlang $x_{T}$ von $190 \mathrm{~mm}$ stromab des Düsenaustritts positioniert. Die Prandtlsonde war in einem Abstand entlang $y_{T}$ von $100 \mathrm{~mm}$ an der gleichen $x_{T^{-}}$und $z_{T}$-Position wie die Hitzdrahtsonde fixiert. Die Ausrichtung der Prandtlsonde parallel zur Anströmung wurde optisch durchgeführt und unterliegt einem gewissen Fehler. Es werden allerdings für die verwendete Prandtlsonde wie für gängige Prandtlsonden bei Ausrichtefehlern unter $5^{\circ}$ nur sehr geringe Fehler in der ausgewerteten Geschwindigkeit erwartet [78]. Eine Fotografie dieser Anordnung ist in Abbildung 5.1.5(a) zu sehen.

Dieser Vorgang wurde viermal wiederholt, wobei aus den Hitzdrahtmessungen jeweils der Betrag der Geschwindigkeit für 10 unterschiedliche Windkanalge- 
(a)

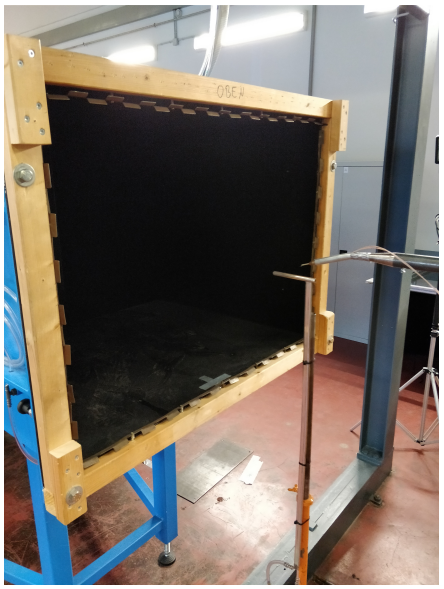

(b)

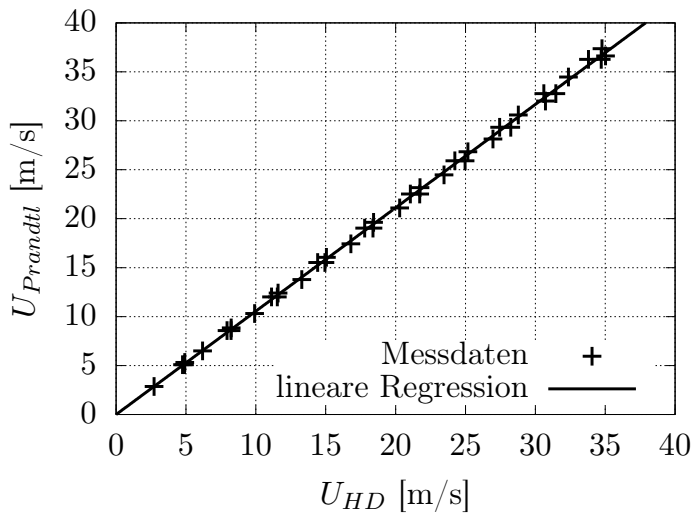

Abbildung 5.1.5: (a) Prandtl- und Hitzdrahtsonde nebeneinander vor der Düse des $1 \mathrm{MG}$ (b) Die mithilfe der Prandtl-Sonde gemessene Geschwindigkeit $U_{\text {Prandtl }}$ in der leeren Messstrecke des Ein-Meter-Kanals in Abhängigkeit vom Geschwindigkeitswert $U_{H D}$ aus der vor dem Kalibrator kalibrierten Hitzdrahtmessung

schwindigkeiten ausgewertet wurde. Die durchgeführten Kalibrierungen und Messungen im Windkanal fanden an unterschiedlichen Messtagen und somit bei verschiedenen Umweltbedingungen statt. In Abbildung 5.1.5(b) sind die beiden unabhängig bestimmten Geschwindigkeitswerte für unterschiedliche Windkanalgeschwindigkeiten und verschiedene Kalibrierungen gesammelt gegeneinander aufgetragen. Mit der Annahme, dass die spannweitigen Geschwindigkeitsunterschiede stromab der Düse des 1MG auf einer Strecke von $100 \mathrm{~mm}$ vernachlässigbar klein sind, muss dieser Vergleich mit einer korrekt kalibrierten Hitzdrahtsonde also eine Gerade mit der Steigung 1 ergeben. Es ergibt sich ein eindeutig linearer Zusammenhang, dessen Steigung aber von 1 abweicht. Mithilfe eines Anpassungsalgorithmus basierend auf der Minimierung der Summe der Fehlerquadrate wurde eine Ursprungsgerade angepasst. Die Steigung dieser Geraden beträgt $k_{c a l}=1.056(3)$. Der angegebene Fehler entspricht der Ungenauigkeit der Steigung der angepassten Ursprungsgerade. Die mit der kalibrierten Hitzdrahtsonde gemessenen Geschwindigkeiten sind also systematisch niedriger als die mit der Prandtlsonde gemessenen.

Dies weist darauf hin, dass der anhand von Gleichung 5.1.29 bestimmte Wert für $U_{2}$ bei der Kalibrierung systematisch zu gering war. Möglicherweise 
wich die Temperatur im Zentrum der Beruhigungskammer des Kalibrators signifikant von der Temperatur des Temperatursensors an der Wand ab. Um einen höheren Wert von $U_{2}$ zu begründen, müsste die Temperatur $T_{1}$ systematisch größer als der gemessene Wert sein. Dies hätte auch eine Auswirkung auf den berechneten Wert von $T_{2}$ und damit auf die angewandte Temperaturkompensation. Ein weiterer Erklärungsansatz betrifft die thermischen Eigenschaften des Wärmetransports durch erzwungene Konvektion. Wäre die Wärmleitfähigkeit der Luft im Kalibrator gegenüber der im Windkanal zirkulierenden Luft erhöht, könnte dies dazu führen, dass bei gleichem Geschwindigkeitsbetrag vor dem Kalibrator eine höhere Anemometerspannung gemessen wird. Dies ist denkbar, da die Druckluft, welche zum Betrieb des Kalibrators verwendet wird, mithilfe eines Kompressors komprimiert wird und möglicherweise Ölpartikel enthält, welche die Wärmeleitfähigkeit der Luft erhöhen.

Für eine abschließende Klärung der Ursache für den Wert $k_{c a l}>1$ wären umfangreichere Untersuchungen nötig. Stattdessen wurde bei der Kalibrierung die kalibrierte Geschwindigkeit $U_{C}$ empirisch linear korrigiert, da der beobachtete Zusammenhang eine klare Linearität aufweist und reproduzierbar ist:

$$
U_{C}=k_{c a l} U_{2}
$$

\subsubsection{Durchgeführter Ablauf der Kalibrierung}

Um für die Auswertung der Hitzdrahtmessungen die Interpolationsmethode verwenden zu können, wurde in den Kalibrierungen dieser Arbeit immer eine zweidimensionale Variation von Anströmgeschwindigkeit und -winkel durchgeführt. Dabei wurde ein Winkelbereich von $-30^{\circ}<\phi_{S o}<+30^{\circ}$ in Schritten von $1^{\circ}$ und ein Geschwindigkeitsbereich von etwa $3 \mathrm{~m} / \mathrm{s}<U<35 \mathrm{~m} / \mathrm{s}$ in 10 Schritten kalibriert. Bei früheren Experimenten im neu ausgelegten Prinzipexperiment $[68,70]$ wurde die Sonde zur Kalibrierung aus dem Haltearm ausgebaut und in einer separaten Traversiervorrichtung vor einem Hitzdrahtkalibrator kalibriert. Dabei sind aber Fehler in der Ausrichtung sowie in den Kontaktwiderständen der benötigten Steckverbindungen nicht zu vermeiden.

Daher wurde für die Kalibrierungen dieser Arbeit der Hitzdrahtkalibrator so auf einer Plattform unter der Modellplatte aufgestellt, dass für die Variation des Anströmwinkels der Sonde die Traversiereinrichtung benutzt werden konnte. Dadurch musste die Sonde für eine Kalibrierung nicht aus dem Haltearm ausgebaut und kein elektrischer Kontakt geöffnet werden. Nach 


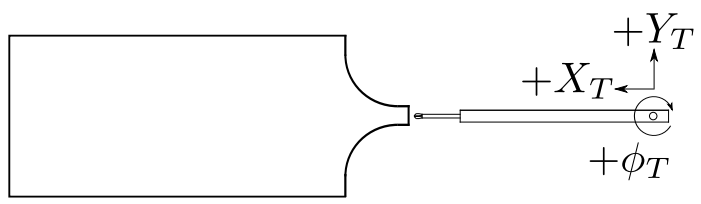

Abbildung 5.1.6: Aufsichtsskizze der V-Sonden-Kalibrierung mit dem Hitzdrahtkalibrator und der Traversiereinrichtung

abgeschlossener Kalibrierung wurde der Hitzdrahtkalibrator wieder von der Plattform entfernt, um bei Messungen im Windkanal keine Versperrung darzustellen. Die Anordnung während der Kalibrierung wird in der Skizze 5.1.6 verdeutlicht.

Dadurch, dass die Drähte der V-Sonde um die Länge des Haltearms von der Drehachse der Traversiereinrichtung entfernt waren, musste bei einer Drehung um $\phi_{T}$ die Position der Traversiereinrichtung in $x_{T}$ und $y_{T}$ korrigiert werden, damit die Sonde in ihrer Kalibrierposition mittig und nah am Düsenaustritt des Kalibrators fest stand während nur der Anströmwinkel variiert wurde. Da die Korrekturstrecken in $x_{T}$ - und $y_{T}$-Richtung stark verschieden und abhängig vom aktuellen Anströmwinkel waren, musste bei jedem Schritt dabei auch die Schrittmotor-Geschwindigkeit der zu verfahrenden Achsen angepasst werden, damit auch zwischenzeitlich die Sonde nicht mit der Düse kollidierte. Mithilfe der Programmierumgebung „LabVIEW“ wurde ein Programm entwickelt, das über eine Steuerspannung am Druckregelventil einen konstanten Geschwindigkeitsbetrag der Anströmung einstellte und dann die Traversiereinrichtung so ansteuerte, dass die Sonde über den definierten Winkelbereich geführt wurde. Pro Winkelschritt wurde nach der Traversierung und vor Aufnahme der Messdaten $1 \mathrm{~s}$ gewartet. Die Messdauer an jeder Position betrug $2 \mathrm{~s}$. Nach der Messung an allen Anströmwinkeln bei konstanter Anströmgeschwindigkeit wurde die Sonde wieder auf den anfänglichen Anströmwinkel rotiert und die Geschwindigkeit erhöht. Für das Einstellen eines stationären Zustands des statischen Drucks in der Vorkammer und der Austrittsgeschwindigkeit wurde nach dem Erhöhen der Steuerspannung eine Wartedauer von $60 \mathrm{~s}$ eingehalten. Der Vorgang wurde dann für die restlichen definierten Anströmgeschwindigkeiten wiederholt. Die Kalibrierung mit $10 \cdot 61=610$ Messpositionen dauerte etwa eine Stunde und enthielt alle Informationen, um beide beschriebenen Auswertemethoden anwenden zu können. Um die Alterung der Hitzdrähte ausreichend zu berücksichtigen, wurde diese Kalibrierung jeweils zu Beginn jedes Messtages wiederholt. 


\subsubsection{Messablauf}

Für die räumliche dreidimensionale Untersuchung der Grenzschichtströmung wurden rasterförmige Hitzdrahtmessungen durchgeführt, bei denen die Hitzdrahtsonde an mehreren Plattentiefenpositionen spannweitig und wandnormal traversiert wurde. Dadurch, dass die beiden Drähte sich an unterschiedlichen spannweitigen Positionen befinden, können die unkorrigierten Messdaten zu Fehlinterpretationen bzgl. spannweitiger Gradienten der Strömungsgrößen führen. Daher wurde die spannweitige Schrittweite so gewählt, dass das Korrekturverfahren für spannweitige Gradienten der zeitlich gemittelten Geschwindigkeitskomponenten nach Deyhle [21] möglich ist. Dieses Verfahren wird im Folgenden beschrieben.

Bezeichnet $i$ den Index der spannweitigen Traversierung, wird die Sonde so traversiert, dass der linke Draht sich im Schritt $i+1$ an der spannweitigen Position befindet, an der sich im vorhergehenden Schritt $i$ der rechte Draht befand, siehe Abb. 5.1.7. Somit kann die Spannung des rechten Drahtes aus Schritt $i$ und die Spannung des linken Drahts aus dem Schritt $i+1$ als ein Spannungspaar an derselben spannweitigen Position interpretiert werden, was ein effektives Korrekturverfahren für die Auswirkung der endlichen Ausdehnung der V-Draht-Sonde auf zeitlich gemittelte Messdaten ergibt. Das Signal des linken Drahtes der ersten spannweitigen Position und das des rechten Drahtes der letzten Position können nicht verwendet werden. Wurde an insgesamt $Z$ spannweitig verteilten Positionen gemessen, liegen korrigierte Daten an $Z-1$ virtuellen Positionen vor, deren absolute spannweitige Koordinaten um die halbe Schrittweite verschoben sind. Eine solche spannweitige Traversierung ist in Abbildung 5.1.7 schematisch dargestellt. Die Hitzdrahtsonde ist bei allen Messungen parallel zur $x_{T}$-Achse ausgerichtet und wird in einem großen $x_{c}$-Bereich im Wesentlichen frontal angeströmt, da die Grenzschichtrandstromlinie dort ebenfalls nahezu parallel zur $x_{T}$-Achse verläuft. Für die beschriebene Korrektur muss die Sonde also pro Schritt in $y_{T}$-Richtung um den Abstand $y_{S o}$ der Drahtmitten traversiert werden, was aufgrund des geometrischen Schiebewinkels $\phi_{g, \infty}=-45.0^{\circ}$ einer Schrittweite in $y_{c}$-Richtung von $\Delta y_{c}=y_{S o} \cdot \sqrt{2}$ entspricht.

Wird die Sonde hingegen in jedem Schritt nur um $\Delta y_{T}=\frac{1}{2} y_{S o}$ traversiert, entspricht die Position des linken Drahtes erst im Schritt $i+2$ der Position des rechten Drahtes in Schritt $i$ und die beschriebene Korrektur lässt sich ebenfalls durchführen. Wurde an insgesamt $Z$ spannweitig verteilten Positionen gemessen, liegen dann korrigierte Daten an $Z-2$ Positionen 


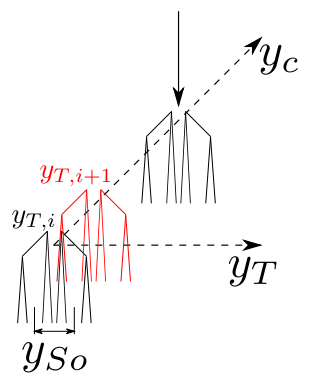

Abbildung 5.1.7: Relevante geometrische Verhältnisse für die Korrektur spannweitiger Gradienten

vor. Der Abstand $y_{S o}$ zwischen den Drahtmitten der verwendeten V-DrahtSonden wurde optisch bestimmt und entspricht $y_{S o}=1.40(3) \mathrm{mm}$ (Der angegebene Fehler entspricht der Auflösung des Messverfahrens). Wenn nicht anders genannt, wurde für die spannweitige Traversierung die Schrittweite $\Delta y_{T}=\frac{1}{2} y_{S o}=0.7 \mathrm{~mm}$ gewählt, was im plattenfesten Koordinatensystem $\Delta y_{c}=0.99 \mathrm{~mm}$ entspricht.

Wie zu Beginn des Abschnitts 5.1 beschrieben wurde, ist die Position der Hitzdrahtsonde zunächst nur im Koordinatensystem der Traversiereinrichtung bekannt, welches durch $x_{T}, y_{T}$ und $z_{T}$ aufgespannt wird. Die Koordinaten $x_{T}$ und $y_{T}$ wurden anhand des geometrischen Schiebewinkels $\phi_{g}=-45.0^{\circ}$ und der bekannten modellfesten Koordinaten eines Bezugspunktes in das modellfeste Koordinatensystem überführt. Als ein solcher Bezugspunkt diente eine Druckbohrung mit sehr genau bekannten Koordinaten, über welcher die Hitzdrahtsonde zum Abgleich der Koordinatensysteme positioniert wurde. Da nur im ebenen Bereich der Modellplatte $\left(x_{c} / c \geq 0.1\right)$ gemessen wurde, waren die vertikale Komponente $z_{T}$ und die wandnormale Komponente $z$ im Rahmen der Ausrichtungsgenauigkeit stets parallel. Zur Überführung ist der Wert der Wandposition in $z_{T}$ nötig. Die Bestimmung dieses Wertes ist mit der nötigen Genauigkeit mithilfe wandnormal verteilter Geschwindigkeitsmessungen in der Grenzschicht möglich. Die Wandposition wurde durch lineare Extrapolation des Geschwindigkeitsprofils in Wandnähe bestimmt. Wie in Abschnitt 5.1 beschrieben, führen die mechanischen Vibrationen der Hitzdrahtsonde zu einem minimal einstellbaren Wandabstand von etwa $z \approx 60 \mu \mathrm{m}$.

Wenn nicht anders erwähnt, wurde für spannweitig und wandnormal rasterförmige Flächenmessungen an fester Profiltiefenposition eine spannweitige Strecke von $L=59.4 \mathrm{~mm}$ an 61 Positionen abgetastet. An jeder spannweitigen 
Position wurde ein wandnormales Profil mit 30 Messpositionen vermessen, wobei die Sonde sich stets der Wand näherte und vor der nächsten spannweitigen Traversierung wieder zum größten Wandabstand traversiert wurde. Dabei wurde eine variable wandnormale Schrittweite gewählt, um die starken Gradienten in Wandnähe besser aufzulösen. Der maximale Wandabstand wurde an die lokale Grenzschichtdicke angepasst. An jeder einzelnen Position wurde das Spannungssignal der beiden Drähte, sowie die Kanalmessgrößen und das Signal der Beschleunigungssensoren mit einer Abtastrate von $32768 \mathrm{~Hz}$ für eine Dauer von $t=4 \mathrm{~s}$ erfasst, nachdem nach der Traversierung eine Wartezeit von $1 \mathrm{~s}$ eingehalten wurde. Eine solche Messung nimmt trotz automatisierter Traversierung etwa vier Stunden pro Messebene und somit pro Profiltiefenposition in Anspruch.

Bei der systematischen Variation der jeweiligen Anregungsstärke der Heizund Rotationsaktuierung war die Bestimmung der Kontrollmodenamplitude das vorrangige Ziel. Daher wurden weniger zeitaufwendige spannweitige Linienmessungen bei dem konstantem Wandabstand durchgeführt, an dem die maximale Amplitude der Kontrollmode beobachtet wurde. Dabei wurde dieselbe spannweitige Schrittweite gewählt wie oben genannt. Um den Zeitaufwand für solche Linienmessungen weiter zu reduzieren, wurde die Messdauer pro Schritt auf $t=2 \mathrm{~s}$ reduziert.

Des Weiteren wurde die Sonde bei einem konstanten Wandabstand knapp außerhalb der Grenzschicht in $x_{T}$-Richtung traversiert. Mit der Annahme unendlich schiebender Bedingungen sind spannweitige Gradienten in der stationären Grundströmung vernachlässigbar, sodass eine Messung in $x_{T^{-}}$ Richtung äquivalent mit einer Messung in $x_{c}$-Richtung ist und man somit die Grenzschichtrandgeschwindigkeiten $u_{c, e}\left(x_{c}\right)$ und $v_{c, e}\left(x_{c}\right)$ erhält.

\subsubsection{Datenerfassung}

Für die rasterförmigen Hitzdrahtmessungen wurde die von Kai Ehrensperger entwickelte Software „SAMS“ (Software für automatische Messdatenerfassung und Schrittmotorsteuerung) [31] eingesetzt, die es ermöglicht, basierend auf einer Liste von geplanten Schritten in $x_{T^{-}}, y_{T^{-}}$und $z_{T^{-}}$Koordinaten eine automatisierte Traversierung und anschließende Datenerfassung durchzuführen.

Die Windkanalsensorik umfasst die Messung des statischen Drucks in der Vorkammer, der Luftfeuchtigkeit in der Vorkammer, der Temperatur in der Vorkammer und des statischen Drucks im engsten Querschnitt der Düse. Der 
Wechselspannungsanteil der Anemometerspannungen wurde vom Gleichspannungsanteil getrennt erfasst und vor der Erfassung mithilfe eines Messverstärkers „Krohn\&Hite Q3916B“ mit passenden Filterkarten „Q38“ verstärkt. Die drei Signale des Beschleunigungssensors für die drei Raumrichtungen wurden ebenfalls vor der Erfassung verstärkt.

Die digitale Erfassung der analogen Spannungssignale erfolgt mithilfe eines Datenerfassungsgeräts „NI-USB-6353“ des Herstellers „National Instruments“, dessen integrierter Analog-Digital-Wandler eine Auflösung von 16 Bits besitzt. Bei einem Messbereich von $-10 \mathrm{~V}<E<10 \mathrm{~V}$ gibt der Hersteller eine nominelle Auflösung von $\Delta E=3.2 \cdot 10^{-4} \mathrm{~V}$ und eine absolute Genauigkeit von $\epsilon_{E}=1.52 \cdot 10^{-3} \mathrm{~V}$ an.

\subsubsection{Fehlerbetrachtung für Hitzdrahtmessungen}

Hitzdrahtanemometrie ist ein indirektes Messverfahren. Aus den gemessenen Anemometerspannungen wird durch Anwendung einer Kalibrierung eine Geschwindigkeit und bei Mehrdrahtsonden auch eine Anströmungsrichtung bestimmt. Dabei sind viele Fehlerquellen zu beachten, deren Einflüsse möglichst minimiert werden müssen. Bei der Erfassung der Anemometerspannungen ist die Genauigkeit des Datenerfassungsgeräts relevant. Da die Anemometerspannung in direktem Zusammenhang mit dem Widerstand des jeweiligen Hitzdrahtes steht, sind die Kontaktwiderstände der Anschlusskabel bis zum eigentlichen Hitzdraht ebenfalls von Bedeutung. Für die Genauigkeit der instationären Geschwindigkeitsinformationen, welche im Wechselspannungsanteil der Anemometerspannungen enthalten sind, ist auch eine Ungenauigkeit des Messverstärkers zu beachten. Die größten Fehler werden aber bei der Anwendung der Kalibrierung auf die Messung erwartet, da hier alle bisher diskutierten Fehlerquellen der Messkette kombiniert auftreten und die Kalibriersituation auf die Messsituation übertragen wird. Alle signifikanten Unterschiede zwischen der Kalibriersituation und der Messsituation führen also zu einer Fehlinterpretation der Anemometerspannung. Eine Alterung der Drähte in der Zeitspanne zwischen Kalibrierung und Messung hat einen Einfluss auf den Drahtwiderstand und schränkt die Anwendbarkeit der Kalibrierung ebenso ein wie eine Änderung der Anschlusskontaktwiderstände. Der Temperaturunterschied wird in der Temperaturkompensation (siehe Abschnitt 5.1.1) berücksichtigt. Die Temperatur während der Messung wird allerdings mithilfe der Kanalmesstechnik in der Beruhigungskammer des Windkanals bestimmt. Eine als adiabatisch angenommene Temperaturände- 
rung durch den Druckabfall zwischen Beruhigungskammer und Messstrecke wird berücksichtigt, aber die ermittelte Temperatur muss nicht exakt mit der Temperatur am Ort der Hitzdrahtsonde übereinstimmen. Der dadurch erwartete Fehler ist normalerweise klein, mit Ausnahme der Untersuchungen mit dem Heizaktuator, siehe Abschnitt 7.3. Ein weiterer möglicher Fehlereinfluss betrifft die Bestimmung der $v_{S o}$-Komponente, also der Komponente senkrecht zur Hauptachse der Sonde, in einer Grenzschicht mit starken wandnormalen Geschwindigkeitsgradienten. Ist der Rollwinkel der Sonde nicht genau so ausgerichtet, dass die beiden Drähte sich in exakt demselben Wandabstand befinden, können wandnormale Geschwindigkeitsgradienten fälschlicherweise als ein Betrag von $v_{S o}$ interpretiert werden. Da der Rollwinkel der Sonde manuell ausgerichtet wird, unterliegt er einer nicht zu vernachlässigenden Ausrichtungsungenauigkeit. Dies ist besonders auch bei der Bestimmung von $v_{s}$ von Bedeutung.

Die beschriebenen Unterschiede zwischen Kalibrier- und Messsituation wurden durch experimentelle Maßnahmen minimiert, was u.a. durch Vergleichmessungen mit einer Prandtlsonde überprüft wurde, siehe Abschnitt 5.1.2. Des Weiteren werden in Abschnitt 6.1 Reproduktionsmessungen der Strömungsgrößen am Grenzschichtrand beschrieben, anhand deren Schwankungsbreiten das Maß des kombinierten Einflusses der beschriebenen Fehler abgeschätzt wird. Bei der Anwendung des kalibrierten nichtlinearen Zusammenhangs zwischen Spannung und Geschwindigkeitskomponenten sind methodische Fehler aber unvermeidbar. Die in Abschnitt 5.1.1 beschriebenen Kalibrierund Auswerteverfahren stellen modellierte Abbildungen zwischen den gemessenen Anemometerspannungen und der Anströmgeschwindigkeit sowie des Anströmwinkels dar. Diese modellierten Abbildungen unterliegen selbst einer gewissen Ungenauigkeit. Des Weiteren weisen sie ein nicht-triviales Verhalten bzgl. der Fehlerfortpflanzung auf. Im Folgenden werden die dadurch erwarteten Fehler diskutiert.

\section{Methodische Fehler - Empirische Betrachtung}

Die Anwendung der Kalibrierung mithilfe der jeweils modellierten Abbildung der gemessenen Anemometerspannungen $E_{1}$ und $E_{2}$ auf die Anströmgeschwindigkeit $U$ und den Anströmungsschiebewinkel $\phi_{S o}$ kann nicht fehlerfrei erfolgen. Wie in Abschnitt 5.1.3 beschrieben wurde, wird die Hitzdrahtsonde bei der Kalibrierung bekannten Anströmgeschwindigkeiten $U_{C}$ und Anströmungsschiebewinkeln $\phi_{S o, C}$ ausgesetzt und jeweils das Spannungspaar $\left(E_{1}, E_{2}\right)$ gemessen. Wendet man die dadurch modellierte Abbildung des Span- 
(a) Geschwindigkeitsfehler

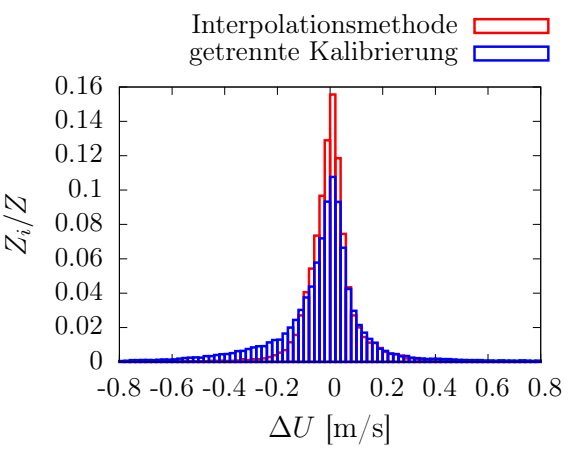

(b) Winkelfehler

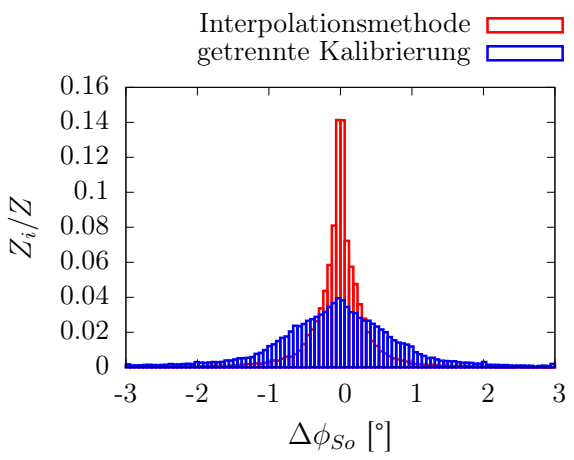

Abbildung 5.1.8: (a) Relative Häufigkeit $Z_{i} / Z$ der Abweichung $\Delta U$ und (b) der Abweichung $\Delta \phi_{S o}$ des jeweils ausgewerteten Werts vom bekannten Wert als Maßs für den Geschwindigkeitsfehler und den Winkelfehler

nungspaares nach den beiden in Abschnitt 5.1.1 beschriebenen Verfahren an, müssten idealerweise dieselben Werte $U_{C}$ und $\phi_{S o, C}$ als Ergebnis erreicht werden. Dies ist aber durch die Ungenauigkeit der jeweils modellierten Abbildung nicht immer der Fall.

Um die Genauigkeit des jeweiligen Kalibrier- und Auswertungsverfahrens zu beurteilen, wurden die während einer Kalibrierung aufgenommenen Spannungen $E_{1}$ und $E_{2}$ als Messwerte interpretiert und mit beiden Methoden ausgewertet. Die Kalibrierung erfolgte jeweils an 61 Werten für $\phi_{S o, C}$ und 10 Werten für $U_{C}$, also an $61 \cdot 10=610$ Messpunkten, von denen für die getrennte Methode nur ein kleiner Teil zur Kalibrierung benötigt wird (10 Messpunkte zur Kalibrierung der Geschwindigkeitsabhängigkeit bei festem Anströmwinkel und 61 Messpunkte zur Kalibrierung der Winkelabhängigkeit bei fester Anströmgeschwindigkeit). Die ausgewerteten Anströmgeschwindigkeiten $U$ und Anströmwinkel $\phi_{S o}$ können dann mit den aus der Kalibrierung bekannten Werten $U_{C}$ und $\phi_{S o, C}$ verglichen werden. Dies wurde für 146 Kalibrierungen mit je 610 Messpunkten durchgeführt (Gesamtanzahl $Z=146 \cdot 610=89060$ ).

Die relative Häufigkeit der Abweichung des ausgewerteten Geschwindigkeitswertes vom bekannten Wert $\Delta U=U-U_{C}$ ist als Histogramm für beide Kalibrier- und Auswertemethoden in Abbildung 5.1.8(a) dargestellt. In Abbildung 5.1.8(b) ist die jeweilige relative Häufigkeit der Winkelabweichung $\Delta \phi_{S o}=\phi_{S o}-\phi_{S o, C}$ als Histogramm dargestellt. Die Standardabweichungen 
dieser Verteilungen sind in Tabelle 5.1.2 zusammengefasst.

\begin{tabular}{l|cc} 
& $s(\Delta U)[\mathrm{m} / \mathrm{s}]$ & $s\left(\Delta \phi_{S o}\right)\left[^{\circ}\right]$ \\
\hline getrennte Kalibrierung und Auswertung & 0.20 & 0.86 \\
Interpolationsmethode & 0.11 & 0.46
\end{tabular}

Tabelle 5.1.2: Standardabweichungen der in Abbildung 5.1.8(a) und 5.1.8(b) dargestellten Verteilungen als Geschwindigkeitsfehler und Winkelfehler für die beschriebenen Kalibrationsmethoden

Die Verteilungen der Abweichungen sind erwartungsgemäß um Null verteilt, wobei die Intervalle mit den geringsten Abweichungen die höchste relative Häufigkeit aufweisen. Die Abweichungen $\Delta U$ für die Interpolationsmethode und die getrennte Methode weisen prinzipell ähnliche Verteilungen auf, während besonders bei der getrennten Methode häufiger zu niedrige Geschwindigkeiten ausgewertet werden als zu hohe. Diese Asymmetrie ist bei der Interpolationsmethode deutlich geringer ausgeprägt, was sich auch in der geringeren Standardabweichung der Geschwindigkeitsabweichungen gegenüber der getrennten Methode äußert. Bei der getrennten Kalibrationsmethode treten des Weiteren deutlich häufiger größere Fehler bei der Auswertung des Anströmwinkels $\phi_{S o}$ zu beiden Seiten des wahren Wertes auf. Die Standardabweichung der Winkelfehler im Fall der Interpolationsmethode entspricht nahezu der Hälfte des bei der Kalibrierung verwendeten Winkelschrittes.

Wegen des deutlich geringeren Fehlers bei der Auswertung des Anströmwinkels wurde im Rahmen dieser Arbeit die Interpolationsmethode zur Auswertung der Hitzdrahtmessungen angewandt. Bei der Auswertung wird $U$ mit $\phi_{S o}$ in unterschiedliche Koordinatensysteme projiziert. Der angegebene Messfehler pflanzt sich dadurch unterschiedlich in die jeweiligen Koordinatensysteme fort und wird bei der Diskussion berücksichtigt werden.

\section{Fehlerfortpflanzung - Statistische Betrachtung}

In Abschnitt 5.1.1 wurde das Kingsche Gesetz (Gleichung 5.1.20) eingeführt. Dieser Zusammenhang stellt eine Modellgleichung für die Auswertung von Geschwindigkeiten aus Anemometerspannungen dar. In der in Abschnitt 5.1.1 beschriebenen Interpolationsmethode wird er verwendet, um auf einem nur grob abgetasteten Gitter aus kalibrierten Geschwindigkeiten physikalisch motiviert zu interpolieren. Bei der ebenfalls in Abschnitt 5.1.1 beschriebenen 
getrennten Auswertemethode stellt Gleichung 5.1.5 eine verallgemeinerte Form des Kingschen Gesetzes dar.

Zur Untersuchung der Fehlerfortpflanzung einer Ungenauigkeit in der Spannung $E$ und den angepassten Parametern des Kingschen Gesetzes $A, B$ und $n$ bietet sich eine statistische Untersuchung an. Dazu wurde das Kingsche Gesetz exemplarisch mithilfe eines Anpassungsalgorithmus zur Minimierung der Summe der Fehlerquadrate an einen kalibrierten Spannungsverlauf $E(U)$ angepasst. Dabei ergeben sich Parameter $A, B$ und $n$ und ihre Kovarianzmatrix. Die Kovarianzmatrix enthält exemplarisch die Ungenauigkeit der angepassten Parameter der Modellgleichung, während für die Ungenauigkeit der Spannung $E$ angenommen wird, dass sie der Hälfte der in Abschnitt 5.1.5 angegebenen Aufösung der Datenerfassung entspricht.

Aus dem kalibrierten Wertebereich werden 100 gleichverteilte Mittelwerte der Spannung $E$ ausgewählt. Unter der Annahme, dass Schwankungen der Anemometerspannung normalverteilt sind, werden für jeden dieser Mittelwerte $10^{6}$ zufällige Werte aus einer Normalverteilung um diesen Mittelwert gezogen, deren Standardabweichung der angenommenen Ungenauigkeit der Spannung $\Delta E=\frac{3 \cdot 2 \cdot 10^{-4}}{2} \mathrm{~V}$ entspricht. Des Weiteren werden für die Parameter $A, B$ und $n$ jeweils $10^{6}$ um ihren jeweiligen Mittelwert normalverteilte Werte zufällig gezogen, wobei die Ziehung aus einer multivariaten Normalverteilung gemäß der Kovarianzmatrix der exemplarisch kalibrierten Parameter des Kingschen Gesetzes durchgeführt wurde. Dabei wurden Methoden aus der Software-Bibliothek NumPy [41] verwendet. Durch Auswertung des Kingschen Gesetzes dieser $10^{6}$ zufällig gezogenen Kombinationen der Eingangsparameter gemäß:

$$
U=\left(\frac{E^{2}-A}{B}\right)^{\frac{1}{n}}
$$

ergibt sich für jeden Mittelwert von $E$ eine statistische Verteilung der $10^{6}$ ausgewerteten Werte von $U$, welche die Nichtlinearität der Modellgleichung berücksichtigt. Diese Art der statistischen Betrachtung einer Fehlerfortpflanzung ist als Monte-Carlo Methode bekannt [19, 48].

In Abbildung 5.1.9(a) sind zwei solcher Verteilungen der ausgewerteten Werte von $U$ am oberen und am unteren Rand des untersuchten Wertebereichs von $\bar{E}$ als Histogramm dargestellt. Dabei erkennt man, dass bei niedrigen Werten von $\bar{E}$ bzw. $\bar{U}$ die Verteilung von $U$ um den Mittelwert im Wesentlichen symmetrisch ist. Bei hohen Mittelwerten weist sie allerdings eine leichte Asymmetrie hin zu höheren Werten auf, während die Schwankungsbreite 
(a)

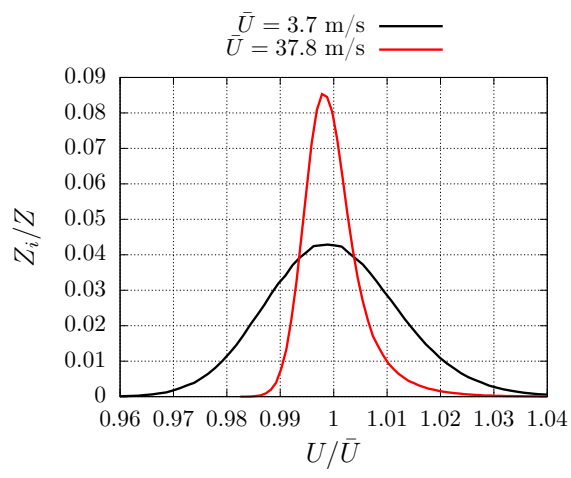

(b)

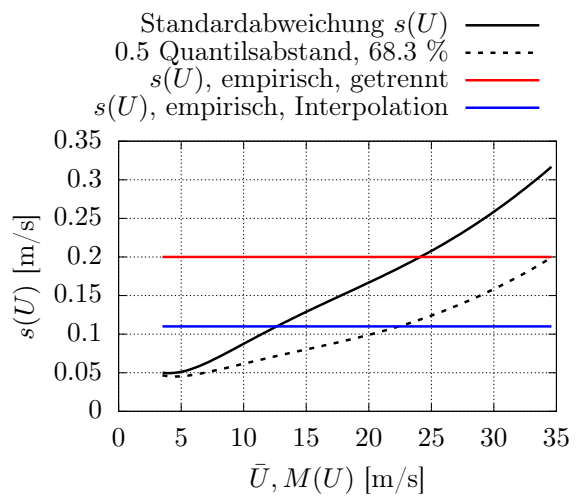

Abbildung 5.1.9: (a) Resultierende Verteilung der ausgewerteten Werte von $U$ für zwei exemplarische Mittelwerte von $E$, (b) Schwankungsbreiten der statistischen Verteilung von $U$ abhängig vom jeweiligen Mittelwert $\bar{U}$ bzw. vom Medianwert $M(U)$ im Vergleich zu den empirisch bestimmten Ungenauigkeiten aus Abschnitt 5.1 .6

relativ zum Mittelwert vergleichsweise geringer ist. Als Maß für diese Schwankungsbreite ist die Standardabweichung der 100 ausgewerteten Verteilungen für $U$ in Abbildung 5.1.9(b) in Abhängigkeit vom jeweiligen Mittelwert $\bar{U}$ der Verteilung und im Vergleich mit den im vorigen Abschnitt empirisch ermittelten Standardabweichungen aus Tabelle 5.1.2 dargestellt. Dort wurde aufgrund der vergleichsweise geringen Anzahl an Stichproben der Geschwindigkeitsfehler nicht abhängig vom Mittelwert der Geschwindigkeit untersucht. Da die Verteilungen im Allgemeinen asymmetrisch sind, sind im Histogramm in einem Intervall mit der Breite der doppelten Standardabweichung mehr Stichproben enthalten als bei einer symmetrischen Verteilung. Daher ist zum Vergleich als alternative Schwankungsbreite der jeweiligen Verteilung auch der halbe Quantilsabstand, in dem 68.3\% der Stichproben enthalten sind, abhängig vom Medianwert $M(U)$ der Verteilung dargestellt. Die ermittelten Schwankungsbreiten dieser statistischen Untersuchung liegen also im selben Wertebereich wie die für die beiden untersuchten Kalibrier- und Auswertemethoden empirisch bestimmten Geschwindigkeitsfehler. Also ergibt sich eine konsistente Abschätzung für die zu erwartenden methodischen Fehler der Hitzdrahtanemometrie. 


\subsection{Der Ein-Meter-Windkanal Göttingen}

Die Grenzschichtuntersuchungen dieser Arbeit wurden am neu ausgelegten Prinzipexperiment der schiebenden ebenen Platte im Ein-Meter-Windkanal Göttingen durchgeführt, wobei es sich um einen umlaufenden Windkanal Göttinger Bauart handelt. Es ist zu beachten, dass der Windkanal seit den Untersuchungen mit dem ursprünglichen Prinzipexperiment erhebliche bauliche Veränderungen erfahren hat. Im Jahr 2015 wurden die Beruhigungskammer, die Düse, der Auffangtrichter und das Axialgebläse ausgetauscht.

Der Windkanal bietet die Möglichkeit, eine offene oder eine geschlossene Messstrecke einzusetzen. Mithilfe des Axialgebläses mit einer elektrischen Leistung von $119 \mathrm{~kW}$ können Strömungsgeschwindigkeiten von maximal $70 \mathrm{~m} / \mathrm{s}$ in der geschlossenen und $65 \mathrm{~m} / \mathrm{s}$ in der offenen Messstrecke erzeugt werden [67]. Die offene Messstrecke mit einer Länge von $2.40 \mathrm{~m}$ kann durch den Einbau eines Zwischenstückes auf eine Länge von $1.50 \mathrm{~m}$ verkürzt werden. Im Rahmen dieser Arbeit wurde jedoch ausschließlich die längere offene Messstrecke verwendet. Der Windkanal verfügt nicht über eine Kühlvorrichtung. Durch die offene Messstrecke findet ein Luftaustausch mit der Umgebungsluft in der umliegenden Halle statt. Durch geöffnete Fenster und eine aktive Belüftung der Halle kann durch den damit verbundenen thermischen Ausgleich die Aufwärmung der im Kanal zirkulierenden Luft begrenzt werden. Die Düse hat eine für den Windkanal namensgebende Breite von $1.0 \mathrm{~m}$ und eine Höhe von $0.74 \mathrm{~m}$.

Für Messungen mit der schiebend eingebauten experimentellen Konfiguration dieser Arbeit wird ein Aufsatz an die Düse angeschlossen, der die Düse so verlängert, dass sie modellparallel abschließt (Abb. 5.2.2(b)). Der gewählte Schiebewinkel der Anströmung beträgt dabei $\phi_{g}=-45.0^{\circ}$, siehe Abschnitt 4.2. In einer Aufsicht hat der Düsenaufsatz einen dreieckigen Querschnitt, dessen Hypotenuse parallel zur Modellvorderkante verläuft. An allen vier Kanten des Düsenaufsatzes befinden sich sog. Seiferth-Flügel am Rand des Austrittsquerschnitts. Diese sollen Rückkopplungseffekte und Resonanzschwingungen der Luftsäule in der Messstrecke vermindern [103]. Der Ein-Meter-Windkanal ist in Abbildung 5.2.1 als Seitenansicht dargestellt. Die experimentelle Konfiguration des neu ausgelegten Prinzipexperiments in der offenen Messstrecke ist in Abbildung 5.2.2(a) als Fotografie dargestellt. Dabei wurde der Düsenaufsatz abgenommen, um die Sicht auf die Konfiguration freizugeben. 


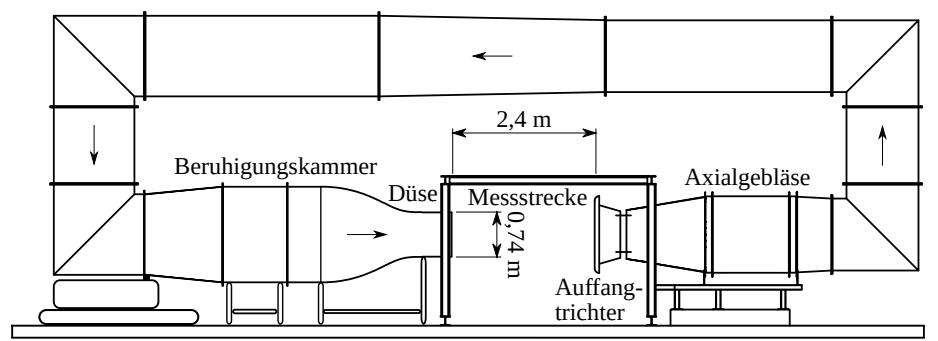

Abbildung 5.2.1: Seitenansicht des Ein-Meter-Windkanals

(a)

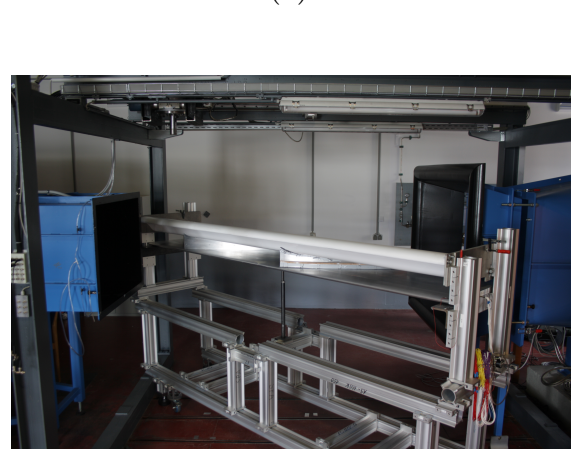

(b)

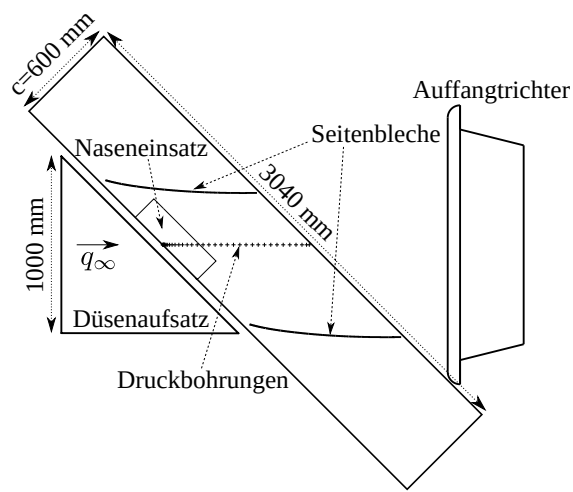

Abbildung 5.2.2: (a) Fotografie der experimentellen Konfiguration im 1MG, ohne Düsenaufsatz (b) Aufsichtsskizze der Modellplatte in der offenen Messstrecke des $1 \mathrm{MG}$

In der Beruhigungskammer des Windkanals sowie in der Düse kurz stromauf des Austritts der Strömung sind Druckbohrungen eingelassen, über die der statische Druck in der Vorkammer und in der Düse gemessen wird. Weiterhin umfasst die Windkanalmesstechnik einen Temperatursensor in der Beruhigungskammer, einen Feuchtesensor und eine Druckdose zur Messung des Umgebungsdrucks in der Halle. Der Windkanal wird über eine Software gesteuert, die aus der Differenz zwischen dem statischen Druck in der Vorkammer und in der Düse den Betrag der aktuellen Geschwindigkeit berechnet [115]. Mithilfe eines Regelalgorithmus kann die Software die Geschwindigkeit über die Leistung des Windkanalantriebs auf einen konstanten Wert regeln. Für die Experimente dieser Arbeit wurde ein Wert von $q_{K, \infty}=25.8 \mathrm{~m} / \mathrm{s}$ als Sollwert gewählt. Dieser Wert entspricht aber aufgrund der starken Verdrängungs- 
wirkung des Aufbaus nicht dem Geschwindigkeitsbetrag einer ungestörten Anströmung, wie sie in den numerischen Simulationen während der Neuauslegung untersucht wurde. Daher wird in Abschnitt 6.1.2 beschrieben, wie virtuelle Anströmbedingungen ausgewählt wurden, für welche die experimentell gemessenen Geschwindigkeitskomponenten am Grenzschichtrand und die Wanddruckverteilung mit der numerischen Simulation nachvollzogen wurden.

In Abschnitt 5.2.2 wird die Reproduzierbarkeit der Versuchsbedingungen im Ein-Meter-Windkanal untersucht.

\subsubsection{Charakterisierung der instationären Anströmung}

Für die Anregung laufender QSI und anderer instationärer Störungen in der Modellgrenzschicht sind instationäre Geschwindigkeitsschwankungen in der Anströmung entscheidend. Im Rahmen der Einmessung des Windkanals nach dem Umbau im Jahr 2015 wurde in der leeren kurzen offenen Messstrecke im für diese Arbeit relevanten Geschwindigkeitsbereich zwischen $20 \mathrm{~m} / \mathrm{s}$ und $30 \mathrm{~m} / \mathrm{s}$ abhängig von der Position in der Messstrecke ein Turbulenzgrad der Anströmung zwischen $T u=0.05 \%$ und $T u=0.25 \%$ festgestellt, wobei besonders bei tiefen Frequenzen unter $100 \mathrm{~Hz}$ viel Energie enthalten war [15]. Um die instationären Eigenschaften der Anströmung mit eingebauter Konfiguration in der Messstrecke zu charakterisieren, wurde die kalibrierte Hitzdraht-V-Sonde stromauf von der Modellplatte positioniert und das Signal der Anemometer und der Kanalmessgrößen während einer Messdauer von 300 Sekunden erfasst. Die Sonde befand sich auf der Windkanalachse gemessen $33.3 \mathrm{~mm}$ stromauf vor der Modellplattenvorderkante in einer Höhe, die im ebenen Bereich der Platte einem Wandabstand von $6 \mathrm{~mm}$ entspricht. Aus der Auswertung der Windkanalmessgrößen ergibt sich ein Betrag der Windkanalgeschwindigkeit von $q_{K, \infty}=25.8(6) \mathrm{m} / \mathrm{s}$. Mit der kalibrierten Hitzdrahtsonde wurde an dieser ausgewählten Position des Strömungsfeldes ein gemittelter Betrag der Geschwindigkeit von $\bar{u}=31.13(7) \mathrm{m} / \mathrm{s}$ gemessen.

Ein exemplarischer Ausschnitt der zeitlich aufgelösten Geschwindigkeitswerte aus den Hitzdraht-Messungen ist in Abb. 5.2.3(a) dargestellt. Wie man schon im zeitlichen Verlauf des Geschwindigkeitsbetrags sieht, enthält das Spektrum instationärer Geschwindigkeitsschwankungen in der Anströmung, dargestellt in Abbildung 5.2.3(b), Anteile in einem breiten Frequenzbereich mit besonders hohen Amplituden $u^{\prime}$ bei Frequenzen unter $50 \mathrm{~Hz}$. Der Frequenzbereich $f<100 \mathrm{~Hz}$ ist in einem Ausschnitt vergrößert zu sehen. Dabei fallen diskrete lokale Maxima auf, besonders ausgeprägt eines zwischen $f=10 \mathrm{~Hz}$ und 
(a)

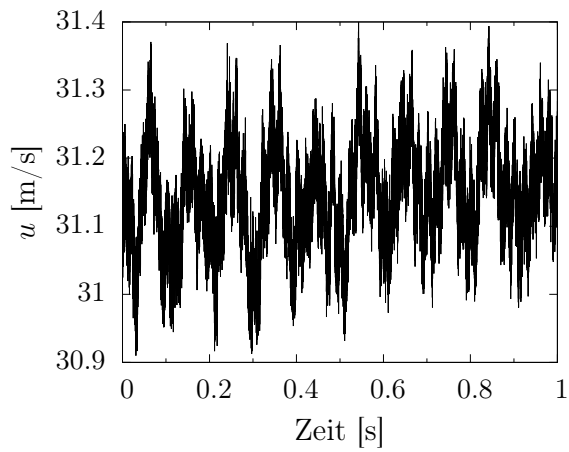

(b)

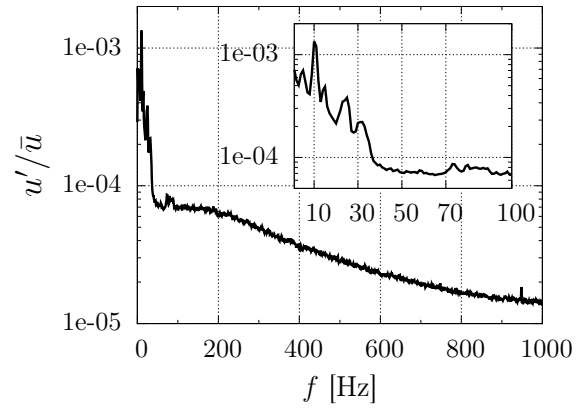

Abbildung 5.2.3: (a) Exemplarischer Ausschnitt aus der ausgewerteten Geschwindigkeit in der Anströmung (b) Geschwindigkeitsspektrum aus dem Hitzdrahtsignal in der Anströmung bis $f=1000 \mathrm{~Hz}$

$f=11 \mathrm{~Hz}$. Ein lokales Maximum bei $f=11 \mathrm{~Hz}$ wurde bereits in Abschnitt $5.1 \mathrm{im}$ exemplarischen Spektrum der Kreuzkorrelation der Geschwindigkeit in der Grenzschicht und dem Signal des Beschleunigungssensors am Hitzdrahthalter festgestellt. Bei der hier diskutierten Messung in der Anströmung stromauf der experimentellen Konfiguration sind im Gegensatz zu Messungen in der Modellgrenzschicht keine starken räumlichen Geschwindigkeitsgradienten zu erwarten (siehe dazu auch Messungen in der freien Anströmung ohne Modell [15]). Tatsächlich sind die Maxima der Kreuzkorrelationsdichte in der Anströmung drei Größenordnungen kleiner als die in Abschnitt 5.1 gezeigten Werte (ohne Abbildung). Daher ist es unwahrscheinlich, dass die beobachteten instationären Schwankungen des Hitzdrahtsignals nur auf eine mechanische Vibration hinweisen, sondern tatsächlich zusätzlich Geschwindigkeitsschwankungen in der Anströmung entsprechen. Als Ursache dieser Geschwindigkeitsschwankungen sind viele Phänomene im Windkanal denkbar. Als Beispiel seien instationäre Ablösephänomene an den Schaufeln des Gebläses oder an den Umlenkschaufeln genannt. Die Geschwindigkeitsschwankungen stellen in der Grenzschicht der Modellplatte eine initiale Anregung für laufende Querströmungsinstabilitäten dar, welche dann gemäß der Stabilitätseigenschaften der Grenzschicht angefacht werden. Da die als Hauptuntersuchungsmethode eingesetzte Hitzdrahtanemometrie langwierige Messungen erfordert, soll im Folgenden untersucht werden, wie sehr sich die Amplitude dieser Geschwindigkeitsschwankungen während eines Experiments verändert. 
(a)

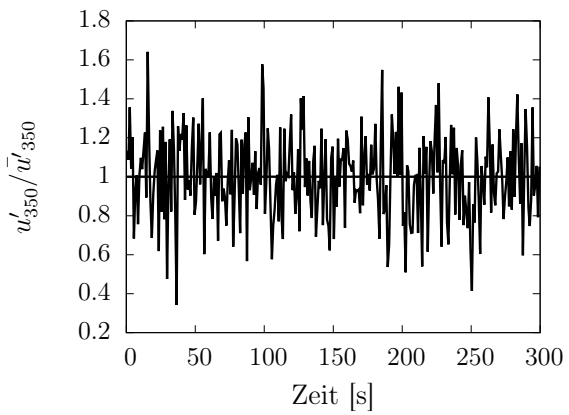

(b)

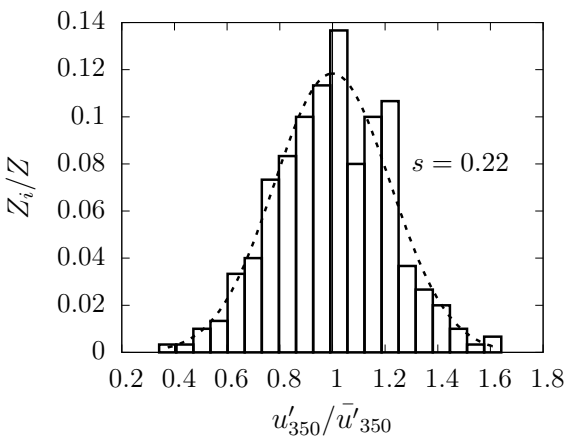

Abbildung 5.2.4: (a) Relative Amplitude der instationären Fluktuationen in der Anströmung bei $f=350 \mathrm{~Hz}$ in jedem zeitlichen Auswertefenster der Länge 1s und (b) die Häufigkeitsverteilung dieser Amplitude als Histogramm

Teilt man die Gesamtdauer der oben beschriebenen Anströmungsmessung in einzelne Fenster einer Länge von jeweils einer Sekunde auf, lässt sich für jedes Fenster ein Spektrum der ausgewerteten Geschwindigkeit berechnen und die Amplitude des spektralen Anteils zu einer bestimmten Frequenz für jedes Fenster auswerten. Nach LST-Ergebnissen werden im Experiment bei etwa $f=350 \mathrm{~Hz}$ stark angefachte laufende QSI erwartet (siehe Abschnitt 6.2.2). Als Maß der relevanten instationären Anregung in der Anströmung ist in Abb. 5.2.4(a) die Amplitude $u_{350}^{\prime}$ der Fluktuationen bei dieser Frequenz relativ zu ihrem Mittelwert dargestellt. Auf der Abszisse ist dabei der zeitliche Mittelpunkt des jeweiligen Fensters aufgetragen. Wie man sieht, schwankt sie um etwa $50 \%$ des Mittelwertes. In Abbildung 5.2.4(b) ist die relative Häufigkeit der ausgewerteten Amplitudenwerte von Geschwindigkeitsschwankungen dieser Frequenz als Histogramm dargestellt. Wie man sieht, ist ihre Häufigkeit nahezu normalverteilt und eine angepasste Normalverteilung ist mit eingezeichnet. Die Standardabweichung dieser Normalverteilung beträgt $s=0.22$. Bei der Interpretation von in der Grenzschicht beobachteten laufenden Querströmungsinstabilitäten muss also berücksichtigt werden, dass das instationäre Anregungsspektrum bzgl. der Frequenz nicht gleichverteilt ist und zudem statistischen Schwankungen unterliegt.

Aus dieser Messung in der Anströmung vor der experimentellen Konfiguration in der offenen Messstrecke lässt sich auch ein Turbulenzgrad bestimmen. Deyhle und Bippes [22] veröffentlichten einen Richtwert für den Turbulenzgrad der Anströmung, bis zu dem mit einer Dominanz stationärer QSI zu 
rechnen ist $(T u=0.15 \%)$. Dies entsprach dem ermittelten Turbulenzgrad des $1 \mathrm{MG}$, welcher aus Hitzdrahtmessungen in der leeren Messstrecke bei $q_{\infty}=19$ $\mathrm{m} / \mathrm{s}$ durch Integration des Geschwindigkeitsspektrums über $2 \mathrm{~Hz}<f<2 \mathrm{kHz}$ bestimmt wurde. Der Turbulenzgrad, der auf gleiche Art und Weise aus der vorliegenden Messung bei $q_{K, \infty}=25.8 \mathrm{~m} / \mathrm{s}$ mit der eingebauten experimentellen Konfiguration bestimmt wurde, beträgt $T u=0.18 \%$. Vernachlässigt man dabei die spektralen Anteile, die signifikant mit dem Signal des am Hitzdrahthalters angebrachten Beschleunigungssensors korrelieren, erhält man einen Turbulenzgrad von $T u=0.13 \%$. Trotz Umbau des Windkanals seit den Messungen von Deyhle und Bippes [22] ist der Turbulenzgrad des 1MG also ähnlich zu den damals gemessenen Werten. Bei diesem Turbulenzgrad ist also damit zu rechnen, dass neben stationären QSI auch laufende QSI mit signifikanter Amplitude in der untersuchten Grenzschicht auftreten. Die Amplituden der dafür besonders relevanten instationären Geschwindigkeitsschwankungen in der Anströmung unterliegen statistischen Schwankungen.

\subsubsection{Reproduzierbarkeit der Strömungsbedingungen}

Da der 1MG über keine Kühlvorrichtung verfügt, unterliegen die Messungen schon wegen der Aufwärmung durch das Gebläse Temperaturänderungen. Durch die offene Messstrecke spielen auch der wetterabhängige atmosphärische Druck, die Temperatur und die Luftfeuchtigkeit der Umgebungsluft eine Rolle für die Versuchsbedingungen. Die Untersuchungen im Rahmen dieser Arbeit fanden im Wesentlichen in zwei Messkampagnen, einer im Sommer (Juli bis August) und einer im Winter (Oktober bis Dezember), statt. Bei jeder Hitzdrahtmessung wurden mithilfe der Windkanalmesstechnik die Temperatur der im Kanal zirkulierenden Luft $T_{K}$, der Umgebungsdruck $p_{\infty}$ und die relative Luftfeuchtigkeit gemeinsam mit den restlichen Messgrößen erfasst. Betrachtet man die zeitlich gemittelten Messwerte dieser Größen an jeder Messposition der Hitzdrahtmessungen als Stichprobe eines stationär und konstant beabsichtigten Zustandes, kann ihre statistische Verteilung über eine große Stichprobenzahl ausgewertet werden, wobei der zeitliche Abstand zwischen den einzelnen Stichproben stark variiert. Die statistische Verteilung der Temperatur $T_{K}$ der im Windkanal zirkulierenden Luft ist für die beiden Messkampagnen getrennt als Histogramm in Abbildung 5.2.5 dargestellt.

Für die Temperatur, die restlichen Messgrößen und daraus abgeleitete Größen ist der jeweils beobachtete Mittelwert mit der zugehörigen Standardabweichung als Fehler in Tabelle 5.2.1 angegeben. Der Mittelwert der aus der 


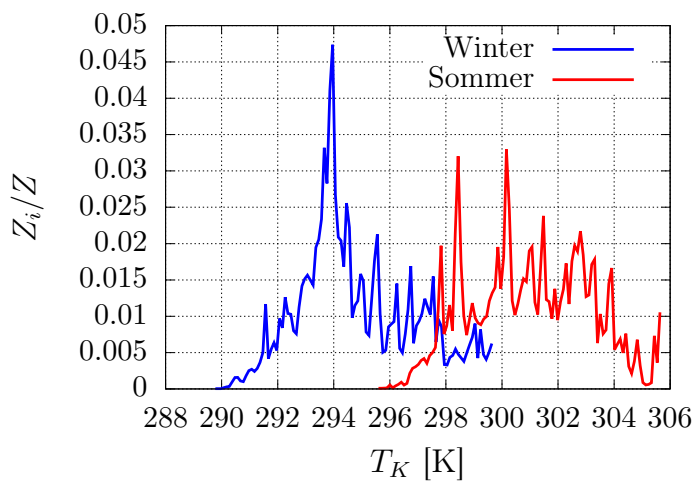

Abbildung 5.2.5: Verteilung der relativen Häufigkeit der Lufttemperatur im Windkanal $T_{K}$ während der beiden Hauptmesskampagnen dieser Arbeit

\begin{tabular}{ccc} 
Größe & Sommer & Winter \\
\hline Stichprobenanzahl $Z$ & 75903 & 109006 \\
$q_{K, \infty}[\mathrm{m} / \mathrm{s}]$ & $25.75(7)$ & $25.73(9)$ \\
$T_{K}[\mathrm{~K}]$ & $301(2)$ & $295(2)$ \\
$p_{\infty}[\mathrm{Pa}]$ & $99800(393)$ & $99900(651)$ \\
rel. Feuchte & $0.43(6)$ & $0.42(6)$ \\
$\mu[\mathrm{Pa} \cdot \mathrm{s}]$ & $1.85(1) \cdot 10^{-5}$ & $1.82(1) \cdot 10^{-5}$ \\
$\rho\left[\frac{\mathrm{kg}}{\mathrm{m}^{3}}\right]$ & $1.15(1)$ & $1.18(1)$ \\
$R e_{K}$ & $1.36(2) \cdot 10^{6}$ & $1.41(2) \cdot 10^{6}$
\end{tabular}

Tabelle 5.2.1: Versuchsbedingungen im $1 M G$ während der beiden Hauptmesskampagnen dieser Arbeit 
statischen Druckdifferenz zwischen Beruhigungskammer und Düse berechneten Anströmgeschwindigkeit $q_{K, \infty}$ weicht leicht vom eingestellten Sollwert $q_{K, \infty}=25.8 \mathrm{~m} / \mathrm{s} \mathrm{ab}$, allerdings um weniger als die Standardabweichung über alle Stichproben. Die Standardabweichung der Temperaturschwankungen während beider Messkampagnen beträgt etwa $2 \mathrm{~K}$, während der Mittelwert der Temperatur $T_{K}$ sich zwischen beiden Messkampagnen um $6 \mathrm{~K}$ unterscheidet. Die beobachteten Schwankungen der Temperatur und des Umgebungsdruckes bedingen Schwankungen in den abgeleiteten Größen der dynamischen Viskosität $\mu$, der Dichte $\rho$ und der wie folgt definierten Windkanal-Reynoldszahl:

$$
R e_{K}=\frac{\rho c q_{K, \infty}}{\mu \cos \left(\phi_{g, \infty}\right)}
$$

Dabei bezeichnet $c$ die Profiltiefe der Modellplatte und $\phi_{g, \infty}=-45^{\circ}$ den geometrischen Schiebewinkel. Diese Reynoldszahl variiert innerhalb der beiden Messkampagnen jeweils um etwa 1.4\% des jeweiligen Mittelwerts und dieser jeweilige Mittelwert unterscheidet sich zwischen den beiden Messkampagnen um etwa 4\%. Leider bietet die Drehzahlregelung des 1MG-Gebläses nur die Möglichkeit, die Anströmgeschwindigkeit $q_{K, \infty}$ näherungsweise konstant zu halten, nicht $R e_{K}$. Die Schwankungen von $R e_{K}$ haben einen Einfluss auf die Störungen in der Modellgrenzschicht und damit auf den untersuchten Transitionsprozess. Dies wird in Abschnitt 7.2.1 anhand von Ergebnissen diskutiert, bei denen dieser Einfluss deutlich auffällt.

\subsection{Druckmessungen}

In der Modellplatte sind zwischen $x_{c} / c=0.25$ und der Hinterkante 19 Druckbohrungen zur Messung des statischen Wanddrucks $p_{W}$ eingelassen. In einem Vorderkanteneinsatz sind zwischen $x_{c} / c=0.0012$ und $x_{c} / c=0.2324$ weitere 21 Druckbohrungen vorgesehen, welche besonders im Bereich der erwarteten Staulinienposition konzentriert wurden, da die Bestimmung der genauen Staulinienlage und des dort aufgestauten Drucks für die numerische Nachbildung des Experiments wichtig ist. Die Bohrungen wurden mit einer computergestützten Bohrmaschine in die Modelle eingelassen, sodass ihre tatsächliche Position mit hoher Genauigkeit der Position gemäß des Entwurfs entspricht.

Für die Messungen der statischen Wanddruckverteilung an der Oberseite der Modellplatte wird dieser Vorderkanteneinsatz in die Modellplatte eingebaut. Die Druckbohrungen im Naseneinsatz und im Rest der Modellplatte sind über 
Leitungen mit einem Scanivalve-System verbunden, welches es ermöglicht, nacheinander alle Druckbohrungen mit einer Differenzdruckdose „Modell 239“ der Firma Setra mit einem Druckmessbereich von $0-500 \mathrm{~Pa}$ zu verbinden. Als Referenzdruck wurde dabei der Umgebungsdruck in der Windkanalhalle verwendet. Nach dem Umschalten des Scanivalve-Systems auf die nächste Druckbohrung wurde zunächst eine Wartedauer von zwei Sekunden eingehalten, bevor die Ausgangsspannung des Druckdosenverstärkers für eine Dauer von 30 Sekunden mit einer Abtastfrequenz von $1000 \mathrm{~Hz}$ aufgenommen und der Mittelwert gespeichert wurde.

Die Angabe des Herstellers zur Genauigkeit der Differenzdruckdose beträgt $\epsilon_{p}=0.14 \%$ der Größe des Druckmessbereichs. Es ergibt sich also eine Unbestimmtheit eines einzelnen Druckmesswertes von $\epsilon_{p}=0.7 \mathrm{~Pa}$. Als relevantes Maß für die Unbestimmtheit der Wanddruckverteilung wird die Streuung über mehrere Reproduktionsmessungen gewählt, siehe Abschnitt 6.1.

\subsection{Infrarot-Thermografie}

Bei den Messungen zur Anregung von stationären QSI mit spannweitig periodischer Wandheizung im 1MG wurden stets stationäre Zustände mit einer stationären Wandtemperaturverteilung untersucht. Diese Wandtemperaturverteilung $T_{W}\left(x_{c}, y_{c}\right)$ an der Oberfläche des Heizaktuators wurde für jeden untersuchten Fall mithilfe einer Infrarotkamera vom Typ „IRCAM Equus 327k L PRO“ mit einer Linse mit einer Brennweite von $25 \mathrm{~mm}$ gemessen. Die Kamera wurde so vor und neben der experimentellen Konfiguration fixiert, dass sie das Infrarotbild der Modelloberfläche durch den Spalt zwischen dem Düsenaufsatz und der Konfiguration aufnehmen konnte (siehe Foto 5.4.1(a)). Mit den zur Verfügung stehenden Objektiven und den Einschränkungen des optischen Zugangs ergibt sich ein gewisser Mindestabstand zur Modelloberfläche. Dieser Abstand und der ebenfalls durch den optischen Zugang eingeschränkte Betrachtungswinkel führen zu einer eingeschränkten räumlichen Auflösung der Modelloberfläche. Es wurde eine räumliche Auflösung von etwa $0.6 \mathrm{pixel} / \mathrm{mm}$ in spannweitiger und $1.6 \mathrm{pixel} / \mathrm{mm}$ in $x_{c}$-Richtung erreicht. Der Abstand zur Oberfläche des Heizaktuator-Naseneinsatzes betrug etwa $800 \mathrm{~mm}$. Die Infrarotkamera ist als Fotografie in ihrer Messposition in Abb. 5.4.1(a) dargestellt. Ein exemplarisches Infrarotbild ist in Abbildung 5.4.1(b) in Falschfarben dargestellt. Dabei ist die Anströmungsrichtung von links, die Vorderkante der Modellplatte ist als Linie von oben links nach unten rechts erkennbar. In Rot bis Weiß erkennt man die Positionen der Modell- 
(a)

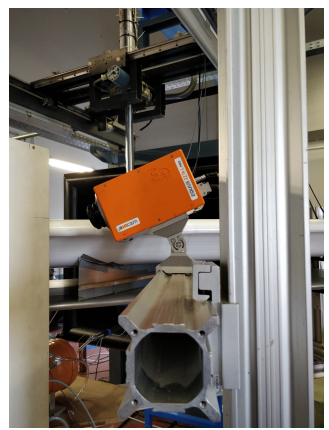

(b)

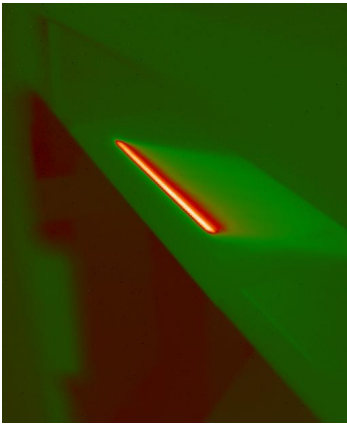

Abbildung 5.4.1: (a) Infrarot-Kamera am Aufbau der experimentellen Konfiguration im 1MG (b) Exemplarisches Infrarotbild in Falschfarben (Rot steht für eine hohe Temperatur, Grün bis Schwarz für eine niedrige Temperatur.)

oberfläche, deren Wandtemperatur durch Heizreihe 3 (siehe Abschnitt 4.5.1) bei einer Leistung von $P=14 \mathrm{~W}$ deutlich angehoben ist. Stromab sind im Nachlauf in hellgrün noch angehobene Wandtemperaturen zu erkennen. Die Infrarotbilder wurden mithilfe der Software „HeatFIT“ von Erich Schülein [102, 109] perspektivisch entzerrt und die Wandtemperaturverteilung wurde in Bezug zu den modellfesten Koordinaten $x_{c}$ und $y_{c}$ ausgewertet.

Die realisierte Auflösung der Infrarotmessungen während der Hitzdrahtmessungen im $1 \mathrm{MG}$ ist ausreichend fein, um die spannweitig gemittelte Wandtemperaturverteilung mean $y_{c}\left(T_{W}\right)\left(x_{c}\right)$ zu bestimmen, allerdings nicht fein genug, um die spannweitige Wandtemperaturperiodizität $\hat{T}_{W}\left(x_{c}\right) \mathrm{zu}$ untersuchen. Daher wurden zusätzliche Infrarotmessungen an dem Heizaktuator-Naseneinsatz in einem Testwindkanal mit kleineren Düsenabmessungen durchgeführt, welche in Abschnitt 7.3.2 diskutiert werden. Dabei kam eine Infrarotkamera vom Typ „FLIR Thermacam SC 500“ zum Einsatz, mit der Aufnahmen der Wandtemperaturverteilung an der Oberfläche des Heizaktuator-Naseneinsatzes aus einem geringeren Abstand von etwa $300 \mathrm{~mm}$ möglich waren, was der minimalen Objektdistanz der Kamera entspricht. Dabei wurde eine räumliche Auflösung von etwa $1.7 \mathrm{pixel} / \mathrm{mm}$ in $y_{c}$-Richtung und $1.5 \mathrm{pixel} / \mathrm{mm}$ in $x_{c}$-Richtung erreicht. Der Hersteller gibt eine absolute Unsicherheit der Temperatur von $\epsilon_{T_{W}}=2 \mathrm{~K}$ und eine Empfindlichkeit von $0.07 \mathrm{~K}$ an. Aus den Aufnahmen wurde für jeden Fall die Amplitude $\hat{T}_{W}$ der beobachteten spannweitigen Wandtemperaturperiodizität als Maximalwert entlang $x_{c}$ ausgewertet, siehe Abschnitt 7.3.2. 


\section{Kapitel 6}

\section{Die transitionelle Strömung im neu ausgelegten Prinzipexperiment}

Ein wichtiges Ziel der Arbeit ist die Neuauslegung eines Prinzipexperiments, in dem die Anwendung der UFD-Transitionsverzögerungsmethode möglich ist und in dem neue Aktuierungskonzepte für diese Methode untersucht werden können. Die Anforderungen an die Neuauslegung wurden in Abschnitt 4.2 beschrieben. In diesem Kapitel wird die experimentell realisierte Strömung im neu ausgelegten Prinzipexperiment charakterisiert und bzgl. dieser Anforderungen geprüft.

Experimentell kam dabei der Naseneinsatz mit Druckbohrungen zum Einsatz, dessen Oberfläche im Gegensatz zu den Aktuatoreinsätzen außer den Druckbohrungen keine besonderen Unregelmäßigkeiten, sondern nur die herstellungsbedingt vorhandene verteilte Rauigkeit besaß. Es wird zunächst in Abschnitt 6.1 gezeigt, dass die unendlich schiebenden Bedingungen im Experiment hinreichend gut erfüllt sind. Im Folgenden wird gezeigt, dass die Wanddruckverteilung und die Grenzschichtrandströmung gut mit den Ergebnissen aus einer numerischen Simulation übereinstimmen. Auf Grundlage der Wanddruckverteilung kann dann eine numerische Grenzschichtgrundströmung bestimmt werden, deren Grenzschichtprofile mit experimentellen Grenzschicht- 
profilen verglichen werden. Diese numerische Grenzschichtgrundströmung wird in Abschnitt 6.2 auf Stabilität untersucht. Dabei kann gezeigt werden, dass die Grenzschichtgrundströmung die bei der Neuauslegung gewünschten Stabilitätseigenschaften besitzt. In Abschnitt 6.3 wird dann gezeigt, dass die experimentell beobachtete Störströmung durch die Stabilitätsergebnisse im Wesentlichen nachvollzogen werden kann.

\subsection{Grundlegende Messungen}

Zur theoretischen Behandlung der Grenzschichtströmung im neu ausgelegten Prinzipexperiment wurde in Abschnitt 2.1 die Annahme unendlich schiebender Bedingungen und ihre Konsequenzen eingeführt. Die Gültigkeit dieser Annahme soll nun in der Strömung an der experimentellen Konfiguration überprüft werden. Des Weiteren soll gezeigt werden, dass die Druckverteilung an der Modellplattenoberseite und die Strömung am Grenzschichtrand sich durch eine numerisch simulierte Umströmung der Konfiguration reproduzieren lassen. Es wird eine numerische Grenzschichtlösung auf Grundlage der gemessenen Wanddruckverteilung eingeführt und überprüft, inwiefern die numerischen Grenzschichtprofile mit experimentellen Profilen übereinstimmen.

\subsubsection{Die Gültigkeit der Annahme unendlich schiebender Bedingungen}

Für die Überprüfung der Gültigkeit der Annahme unendlich schiebender Bedingungen muss gezeigt werden, dass spannweitige Gradienten in der reibungsfreien Außenströmung im Wesentlichen vernachlässigt werden können. Dazu wurde die Hitzdrahtsonde bei $x_{c} / c=0.35$ und $x_{c} / c=0.5$ bei konstantem Wandabstand knapp oberhalb der Grenzschicht entlang einer spannweitigen Strecke von $400 \mathrm{~mm}$ bzw. $900 \mathrm{~mm}$ traversiert. Dabei wurden größere spannweitige Schrittweiten $\Delta y_{c}$ gewählt als in Abschnitt 5.1.4 beschrieben. Zur Verdeutlichung sind diese linienförmigen Messbereiche in der Aufsichtsskizze 6.1.2 der experimentellen Konfiguration in der offenen Messstrecke des 1MG mit eingezeichnet. Der in Abschnitt 5.2 beschriebene Düsenaufsatz ist ebenfalls eingezeichnet, um die Position der Seitenbleche relativ zu den seitlichen Rändern des Freistrahls aus der Düse einzuordnen. Stromab der experimentellen Konfiguration ist der Auffangtrichter mit eingezeichnet, mit dem die Strömung in das Gebläse des 1MG geführt wird, siehe Abschnitt 5.2. 
(a) $x_{c} / c=0.35$

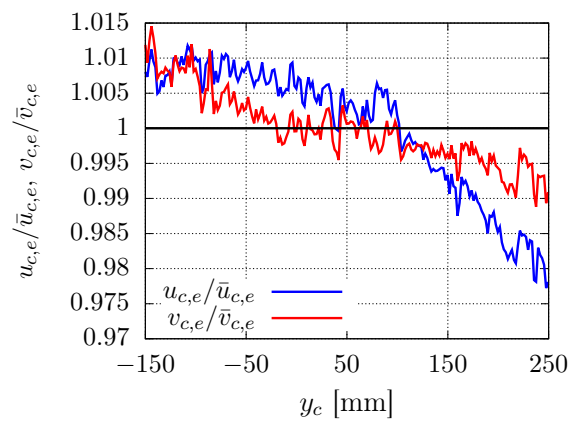

(b) $x_{c} / c=0.5$

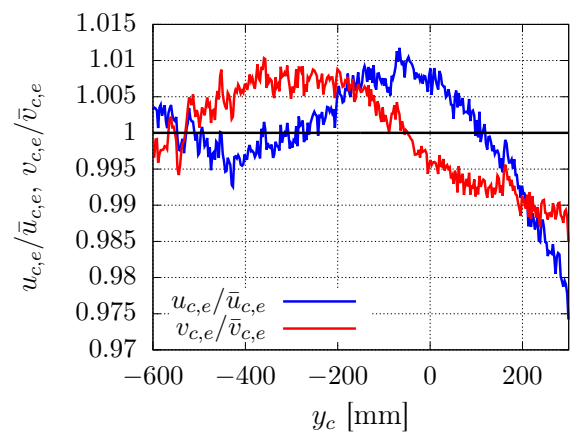

Abbildung 6.1.1: (a) Verhältnis der $u_{c^{-}}$und $v_{c^{-}}$-Geschwindigkeitskomponente am Grenzschichtrand zu ihrem jeweiligen spannweitigen Mittelwert bei konstantem Wandabstand und $x_{c} / c=0.35$ (b) Äquivalente Darstellung für $x_{c} / c=0.5$

Die jeweils ermittelten Geschwindigkeitskomponenten $u_{c}$ und $v_{c}$ wurden in den Abbildungen 6.1.1(a) und 6.1.1(b) durch ihren spannweitigen Mittelwert entdimensionalisiert. Bei der Angabe von Werten für $y_{c}$ wird in diesem und den weiteren Kapiteln der Ursprung so verschoben, dass $y_{c}=0 \mathrm{~mm}$ der spannweitigen Position des Druckschnittes entspricht (siehe Abbildung 4.4.1(b)). Der Druckschnitt verläuft parallel zur Anströmungsrichtung.

An der Position $x_{c} / c=0.35$ wird über die vermessene spannweitige Strecke eine maximale $u_{c}$-Differenz von $2.3 \%$ und eine maximale $v_{c}$-Differenz von $1.5 \%$ vom jeweiligen spannweitigen Mittelwert beobachtet. An der Plattentiefenposition $x_{c} / c=0.5$ wurde eine deutlich größere spannweitige Strecke untersucht. Die Leitbleche befinden sich an dieser Plattentiefenposition bei $y_{c}=-728 \mathrm{~mm}$ und bei $y_{c}=+432 \mathrm{~mm}$. Über die vermessene spannweitige Strecke wird eine maximale $u_{c}$-Differenz von $2.6 \%$ und eine maximale $v_{c^{-}}$ Differenz von $1.5 \%$ vom jeweiligen spannweitigen Mittelwert beobachtet, die beide bei stark positiven Werten von $y_{c}$ auftreten. Im ursprünglichen Prinzipexperiment der schiebenden ebenen Platte wurden auf einer kürzeren spannweitig untersuchten Strecke größere Geschwindigkeitsunterschiede beobachtet [76].

Auffällig ist die Abnahme des Betrags beider Geschwindigkeitskomponenten bei positiven $y_{c}$-Werten. Möglicherweise stellt dies einen Einfluss des spannweitigen Randes der Anströmung dar, siehe Abbildung 6.1.2. Die meisten Messungen im Rahmen dieser Arbeit fanden allerdings zwischen $y_{c}=-250 \mathrm{~mm}$ und 


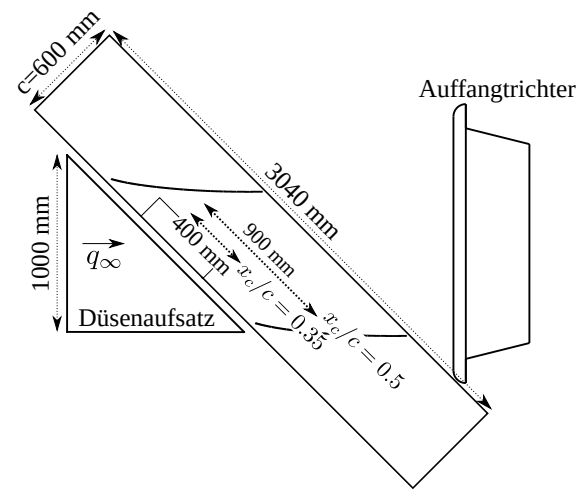

Abbildung 6.1.2: Aufsichtsskizze der Modellplatte in der offenen Messstrecke des $1 \mathrm{MG}$

$y_{c}=+100 \mathrm{~mm}$ statt, wo die Abweichungen der Grenzschichtrandgeschwindigkeiten von ihrem Mittelwert geringer sind als die oben beschriebenen maximalen Abweichungen. Die Auswahl dieses spannweitigen Bereichs liegt darin begründet, dass insbesondere der spannweitige Bereich im Nachlauf der Aktuatoren mit Hitzdrahtmessungen untersucht wurde. Aufgrund des Verlaufs der gekrümmten Potentialstromlinie und der Wirbelachsen der QSI ist der Nachlauf eines Aktuators, dessen spannweitige Ausdehnung im Nasenbereich mittig die Verbindungslinie der Druckbohrungen schneidet $\left(y_{c}=0 \mathrm{~mm}\right)$, bei stromab gelegenen Plattentiefenpositionen zu negativen $y_{c}$-Werten verschoben. Die beobachteten Abweichungen der Geschwindigkeitskomponenten von ihrem jeweiligen Mittelwert werden als gering bewertet. Deshalb sind sie ein Indiz dafür, dass die Vernachlässigung spannweitiger Gradienten bei der theoretischen Behandlung der Grenzschichtgrundströmung eine gerechtfertigte Annahme ist und dass unendlich schiebende Bedingungen ausreichend gut erfüllt sind.

\subsubsection{Strömung am Grenzschichtrand und virtuelle Anströmungsbedingungen}

Die aus der statischen Druckdifferenz zwischen der Windkanalvorkammer und der Windkanaldüse bestimmte Kanalgeschwindigkeit $q_{K, \infty}$ betrug für alle Experimente im Rahmen dieser Arbeit $q_{K, \infty}=25.8 \mathrm{~m} / \mathrm{s}$. Der Schiebewinkel der Anströmung betrug $\phi_{g, \infty}=-45.0^{\circ}$ und die Modellplatte wurde ohne Anstellwinkel ausgerichtet. 
Im Experiment wurde die Wanddruckverteilung $p_{W}\left(x_{c}\right)$ an der Modellplattenoberseite über Druckbohrungen gemessen, wie in Abschnitt 5.3 beschrieben. Die Verläufe von $u_{c, e}\left(x_{c}\right)$ und $v_{c, e}\left(x_{c}\right)$ wurden gemessen, indem die Hitzdrahtsonde knapp außerhalb der Grenzschicht in der Hauptkanalachsenrichtung $-x_{T}$ über einen großen Teil der Profiltiefe der Modellplatte traversiert wurde. Dabei wurden in regelmäßigen Abständen von $\Delta x_{c}=\Delta x_{T} \cdot \cos \left(45^{\circ}\right)=0.01 c$ Daten aufgenommen. Da spannweitige Gradienten vernachlässigt werden können, ist eine Messung entlang $x_{T}$ gleichwertig mit einer Messung entlang $x_{c}$. Diese Messungen wie auch die Messung der Wanddruckverteilung $p_{W}\left(x_{c}\right)$ wurden in jeder Messkampagne mehrfach wiederholt, um die Reproduzierbarkeit der Randbedingungen zu dokumentieren. Dabei bezeichnet eine Messkampagne einen Zeitraum, in dem die experimentelle Konfiguration kontinuierlich in der offenen Messstrecke des $1 \mathrm{MG}$ in unveränderter Position fixiert war. Jeweils zum Anfang einer Messkampagne musste die experimentelle Konfiguration neu in der Messstrecke ausgerichtet werden, wodurch die Reproduzierbarkeit der Strömungsbedingungen potentiell beeinflusst wurde. Durch Mittelung dieser Reproduktionsmessungen konnte die Unsicherheit sowohl der mittleren Druckverteilung als auch der mittleren Geschwindigkeitsverteilungen am Grenzschichtrand deutlich verringert werden. Die Schwankungsbreiten der Reproduktionsmessungen selbst werden zwecks einer Bewertung der Reproduzierbarkeit der Randbedingungen in Abschnitt 6.1.3 separat diskutiert.

Für die Bestimmung der mittleren Wanddruckverteilung wurden acht in unterschiedlichen Messkampagnen gemessene Druckverteilungen $\left(M_{p}=8\right)$ gemittelt. Im Diagramm 6.1.3(a) ist die so gemittelte Wanddruckverteilung $p_{W}\left(x_{c}\right)$ dargestellt. Als Fehlerbalken ist der Fehler des Mittelwertes $\epsilon_{p_{w}}=\frac{s\left(p_{W}\right)}{\sqrt{M_{p}}}$ dargestellt, wobei $s\left(p_{W}\right)$ die Standardabweichung der $M_{p}$ gemessenen Wanddrücke an der jeweiligen Druckbohrung bezeichnet. Die im neu ausgelegten Prinzipexperiment realisierte Druckverteilung besitzt die in Abschnitt 4.2 beschriebenen Eigenschaften der Zieldruckverteilung, einen stark negativen Druckgradienten stromab der Staulinienposition $x_{c} / c=0.013$ und einen schwächeren aber weiterhin negativen Gradienten weiter stromab bis nahezu zum Plattenende.

Zur Bestimmung der mittleren Verläufe der Grenzschichtrandgeschwindigkeiten wurden die Verläufe aus 18 Hitzdrahtmessungen mehrerer Messkampagnen gemittelt $\left(M_{e}=18\right)$. Dabei musste linear auf ein gemeinsames Raster entlang $x_{c}$ interpoliert werden, da die Hitzdrahtmessungen nicht immer auf demselben $x_{c}$-Raster durchgeführt wurden. Die Fehler $\epsilon_{u_{c, e}}$ und $\epsilon_{v_{c, e}}$ des 
(a)

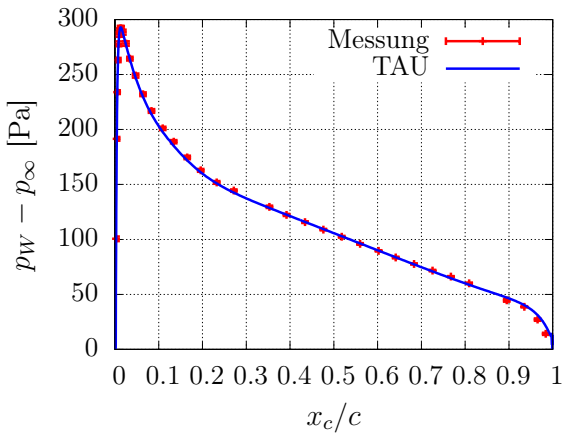

(b)

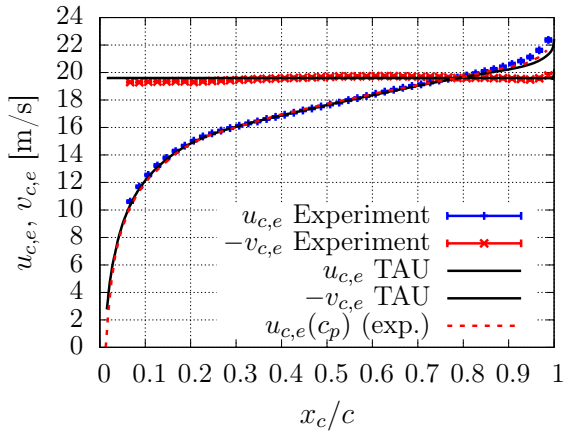

Abbildung 6.1.3: (a) Vergleich der gemessenen mittleren Wanddruckverteilung mit der Wanddruckverteilung in der numerischen TAU-Simulation (mit unterschiedlichem $p_{\infty}$ ), (b) Vergleich der Verläufe von $u_{c, e}$ und $v_{c, e}$ am Grenzschichtrand gemittelt aus Hitzdrahtmessungen im Experiment bei $y_{c}=0 \mathrm{~mm}$ und in der numerischen Simulation (TAU) sowie des $u_{c, e}$-Verlaufs unter Annahme unendlich schiebender Bedingungen gemäß der gemessenen Druckverteilung und Gleichung 2.1 .13

jeweiligen mittleren Geschwindigkeitswertes auf diesem gemeinsamen Raster wurden auf die gleiche Art wie die Fehler der mittleren Wanddrücke aus der jeweiligen Standardabweichung bestimmt und sind als Fehlerbalken des jeweiligen Mittelwerts in Abb. 6.1.3(b) dargestellt. Die dargestellten Fehler $\epsilon_{p_{w}}, \epsilon_{u_{c, e}}$ und $\epsilon_{v_{c, e}}$ des jeweiligen Mittelwertes sind von geringem Betrag und die Fehlerbalken sind ähnlich groß wie typische Symbole. Die Schwankung der jeweiligen Messgröße über die gemittelten Messungen wird in Abschnitt 6.1.3 diskutiert.

Der Verlauf der experimentell bestimmten $u_{c, e}$-Komponente spiegelt die Eigenschaften der Druckverteilung wider. Im Plattentiefenbereich des stark negativen Druckgradienten etwa bis $x_{c} / c=0.2$ wird eine starke Beschleunigung der Strömung in $x_{c}$-Richtung beobachtet. Im weiteren $x_{c}$-Verlauf erfährt die Strömung eine weitere aber schwächere Beschleunigung.

Wie in Abschnitt 2.1 beschrieben, gilt unter unendlich schiebenden Bedingungen, dass der Wert der Geschwindigkeitskomponente $v_{c, e}=v_{c, \infty}$ am Grenzschichtrand entlang $x_{c}$ konstant bleibt (Gleichung 2.1.15). Die geringe Abweichung der am Grenzschichtrand gemessenen $v_{c, e}$-Komponente von ihrem Mittelwert entlang $x_{c}$ von maximal $1.4 \%$ diese Mittelwertes ist ein 
weiteres Indiz dafür, dass die unendlich schiebenden Bedingungen ausreichend gut erfüllt sind. Die Randbedingungen für die Grenzschichtströmung erfüllen also die Ziele der Auslegung.

In Abschnitt 2.1 wurde ebenfalls beschrieben, dass unter unendlich schiebenden Bedingungen diese Randbedingungen für die Grenzschichtströmung über folgende Gleichungen verknüpft sind:

$$
\begin{aligned}
c_{p}\left(x_{c}\right) & =\frac{p_{W}\left(x_{c}\right)-p_{\infty}}{\frac{1}{2} \rho u_{c, \infty}^{2}} \\
u_{c, e}\left(x_{c}\right) & =\sqrt{u_{c, \infty}^{2}\left(1-c_{p}\right)} \\
u_{c, \infty} & =q_{\infty} \cos \left(\phi_{\infty}\right) \\
v_{c, e}\left(x_{c}\right) & =v_{c, \infty}=q_{\infty} \sin \left(\phi_{\infty}\right)
\end{aligned}
$$

Der Index $\infty$ bezeichnet dabei einen ungestörten Anströmungszustand in unendlicher Entfernung stromauf von der experimentellen Konfiguration. Die experimentelle Konfiguration stellt in der offenen Messstrecke des 1MG eine erhebliche Versperrung des Freistrahls der Düse dar. Daher existiert ein solcher ungestörter Zustand nirgendwo in der Messstrecke des Windkanals. Also entspricht die aus der statischen Druckdifferenz zwischen Beruhigungskammer und Düse berechnete Kanalgeschwindigkeit $q_{K, \infty}$ nicht der ungestörten Anströmgeschwindigkeit $q_{\infty}$, der geometrische Schiebewinkel $\phi_{g, \infty}$ nicht dem ungestörten Schiebewinkel $\phi_{\infty}$ und der Umgebungsdruck in der Windkanalhalle nicht dem statischen Druck in der ungestörten Anströmung $p_{\infty}$. Es wird nun nach einem virtuellen ungestörten Anströmungszustand gesucht, definiert durch $q_{\infty}, \phi_{\infty}$ und $p_{\infty}$, für den die gemessene Druckverteilung $p_{W}\left(x_{c}\right)$ und die gemessene Grenzschichtrandgeschwindigkeit $u_{c, e}\left(x_{c}\right)$ über Gleichungen 6.1.1 und 6.1.2 verknüpft werden können, während Gleichungen 6.1.3 und 6.1.4 erfüllt sind. Dabei soll die virtuelle Komponente $v_{c, \infty}$ dem Wert der entlang $x_{c}$ gemittelten experimentell bestimmten $v_{c, e}$-Komponente aus Hitzdrahtmessungen entsprechen. Auch in den Experimenten am ursprünglichen Prinzipexperiment der schiebenden ebenen Platte im 1MG wurde bereits festgestellt, dass eine gute Übereinstimmung zwischen der numerisch simulierten Strömung und der experimentell realisierten Strömung nur für einen leicht vom Experiment abweichenden Schiebewinkel in der numerischen Simulation erreicht wurde, dem sog. effektiven Schiebewinkel [21, 62, 76, 79].

Die virtuellen Anströmbedingungen, für die der mit Gleichung 6.1.2 aus der experimentellen Druckverteilung bestimmte $u_{c, e}$-Verlauf mit dem Verlauf der experimentell durch Hitzdrahtmessungen bestimmten Geschwindigkeitskom- 
ponente gut übereinstimmt und für die $v_{c, \infty}$ dem entlang $x_{c}$ gemittelten Wert von $v_{c, e}\left(x_{c}\right)$ aus Hitzdrahtmessungen entspricht, lauten:

$$
\begin{aligned}
q_{\infty} & =29.09 \mathrm{~m} / \mathrm{s} \\
\phi_{\infty} & =-42.26^{\circ}
\end{aligned}
$$

Nimmt man an, dass eine der Druckbohrungen im Nasenbereich genau der $x_{c} / c$-Position der Staulinie entspricht und damit der maximal gemessene Wanddruck $p_{W}\left(x_{c}\right)$ dem Staudruck entspricht, ergibt sich, dass der virtuelle statische Druck $p_{\infty}$ in der virtuellen ungestörten Anströmung etwa $16 \mathrm{~Pa}$ größer ist als der Referenzdruck der Druckmessung, der Umgebungsdruck in der Windkanalhalle. Der aus der experimentellen Druckverteilung über Gleichung 6.1 .2 bestimmte Verlauf der Geschwindigkeitskomponente $u_{c, e}$ ist zum Vergleich ebenfalls in Abbildung 6.1.3(b) dargestellt. Es zeigt sich insgesamt eine gute Übereinstimmung mit dem experimentell aus Hitzdrahtmessungen bestimmten Verlauf. Für $x_{c} / c>0.9$ sind leichte Abweichungen der beiden Verläufe zu erkennen, die darauf hinweisen, dass die Annahme unendlich schiebender Bedingungen in diesem Bereich verletzt wird.

Diese virtuellen Anströmbedingungen werden auch in einer numerischen quasidreidimensionalen Simulation der Strömung um die experimentelle Konfiguration verwendet, um einen Vergleich der experimentellen Ergebnisse mit numerischen Ergebnissen zu ermöglichen, ohne die voll dreidimensionale Strömung in der offenen Messstrecke zu simulieren. Eine Simulation der voll dreidimensionalen Strömung wäre mit erheblichem numerischen Aufwand verbunden und wurde daher im Rahmen dieser Arbeit nicht durchgeführt. Mithilfe des Strömungslösers TAU (siehe Abschnitt 3.1) wurden mit der ausgelegten Geometrie der Modellplatte, des Verdrängungskörpers und des Vorflügels in der ausgelegten Konfiguration numerische Strömungssimulationen mit einer Fernfeld-Einströmungsbedingung und spannweitig periodischer Randbedingung durchgeführt. Abweichend von den oben beschriebenen virtuellen ungestörten Anströmbedingungen wurde hier ein effektiver Anstellwinkel von $\gamma=-3.80^{\circ}$ verwendet, um die $x_{c}$-Position der Staulinie an die im Experiment beobachtete Position anzupassen. Dementsprechend wurde die ungestörte Anströmgeschwindigkeit um den Faktor $\cos ^{-1}(\gamma)$ auf $q_{\infty}=29.15 \mathrm{~m} / \mathrm{s}$ erhöht, während derselbe Schiebewinkel $\phi_{\infty}=-42.26^{\circ}$ verwendet wurde.

Die Wanddruckverteilung an der Modellplattenoberseite der numerischen Simulation ist im Vergleich zu den experimentellen Werten zusätzlich in Abb. 6.1.3(a) dargestellt. Des Weiteren sind auch die Verläufe der Geschwindigkeitskomponenten am Grenzschichtrand aus der numerischen Simulation 
zum Vergleich mit den experimentellen Werten in Abb. 6.1.3(b) dargestellt. Insgesamt stimmen die Verläufe sehr gut überein, einen Unterschied stellt man allerdings auch nahe der Plattenhinterkante stromab von $x_{c} / c=0.9$ fest. An der Plattenhinterkante tritt die Strömung aus dem Kanal zwischen Modellplatte und Verdrängungskörper aus und stromab der Konfiguration in den Auffangtrichter des 1MG ein. Durch den spannweitig variierenden Abstand zwischen Plattenhinterkante und Auffangtrichter ist es wahrscheinlich, dass dort spannweitige Gradienten auftreten und die Annahme unendlich schiebender Bedingungen ungültig wird. Die gute Übereinstimmung der gemessenen Druckverteilung an der Modellplatte mit der Druckverteilung aus der numerischen Simulation, welche auf der entworfenen Konfiguration basiert, ist ein Indiz für eine ausreichend gute Fertigungsgenauigkeit der einzelnen Komponenten (Modellplatte, Verdrängungskörper und Vorflügel) sowie für eine ausreichend gute Ausrichtungsgenauigkeit dieser zueinander. Zusätzlich ist sie ein weiterer Hinweis auf ausreichend gut erfüllte unendlich schiebende Bedingungen.

\subsubsection{Reproduzierbarkeit der Druckverteilung und Grenzschichtrandströmung}

Die oben beschriebenen Reproduktionsmessungen bieten auch die Gelegenheit, die Schwankungsbreiten der Messergebnisse zu bestimmen und mit den in den Abschnitten 5.3 und 5.1.6 beschriebenen Unsicherheiten der jeweiligen Messmethode zu vergleichen.

Die Schwankungsbreiten der Geschwindigkeitskomponenten am Grenzschichtrand über die $M_{e}=18$ Reproduktionsmessungen enthalten den Einfluss aller in Abschnitt 5.1.6 beschriebenen Fehlerquellen von Hitzdrahtmessungen und zusätzlich Einschränkungen in der Reproduzierbarkeit der Versuchsbedingungen (siehe Abschnitt 5.2.2). Dabei ergibt sich für jede $x_{c}$-Position der Reproduktionsmessungen eine Standardabweichung der bestimmten Geschwindigkeiten bzw. des Geschwindigkeitsbetrags $|U|$ und des Anströmwinkels im sondenfesten Koordinatensystem $\phi_{S o}$. Die über das $x_{c}$-Raster gemittelten Standardabweichungen von $|U|$ und $\phi_{S o}$ am Grenzschichtrand betragen $s(|U|)=0.31 \mathrm{~m} / \mathrm{s}$ und $s\left(\phi_{S o}\right)=0.59^{\circ}$. Diese Werte liegen in derselben Größenordnung wie die in Abschnitt 5.1.6 beschriebenen methodischen Fehlerabschätzungen zu Hitzdrahtmessungen. Dies legt die Interpretation nahe, dass die Schwankungen der Geschwindigkeitskomponenten am Grenz- 
schichtrand aufgrund von veränderlichen Versuchsbedingungen geringer sind als es die Messmethode in der Lage ist, nachzuweisen.

Für jede der 40 Druckbohrungen in der Modellplatte lässt sich die Schwankungsbreite des Wanddruckes an der jeweiligen $x_{c}$-Position durch die Standardabweichung $s\left(p_{W}\left(x_{c}\right)-p_{\infty}\right)$ über die $M_{p}=8$ Wiederholungsmessungen ausdrücken. Der Mittelwert dieser Standardabweichungen beträgt

$$
\overline{s\left(p_{W}\left(x_{c}\right)-p_{\infty}\right)}=4 \mathrm{~Pa} .
$$

Dieser Wert ist größer als die in Abschnitt 5.3 beschriebene Unsicherheit eines einzelnen Druckmesswertes $\epsilon_{p}$, sodass die Einschränkungen in der Reproduzierbarkeit der Versuchsbedingungen bei der Messung der Druckverteilung die angenommene Messunsicherheit überwiegen. Die Reproduzierbarkeit der Versuchsbedingungen wurde in Abschnitt 5.2.2 anhand der Windkanalmessgrößen diskutiert.

\subsubsection{Grenzschichtgrundströmung}

In Abschnitt 2.2 wurde die grundlegende Annahme der linearen Stabilitätstheorie beschrieben, dass der stationären Grundströmung Störungen mit relativ kleinen Amplituden überlagert sind. Der Profiltiefenbereich, in dem die Störungsamplituden ausreichend klein für die Vernachlässigung nichtlinearer Terme sind, wird der lineare Bereich genannt. In diesem Bereich entspricht die zeitlich und spannweitig gemittelte experimentell vermessene Grenzschichtströmung der stationären Grundströmung. Basierend auf der experimentell bestimmten Druckverteilung wurde mithilfe der Software COCO eine numerische Grenzschichtlösung bestimmt (siehe Abschnitt 3.1). Als Anströmbedingungen wurden die im vorangegangenen Abschnitt 6.1.2 beschriebenen virtuellen Anströmbedingungen verwendet. Sie lauten $q_{\infty}=29.09 \mathrm{~m} / \mathrm{s}$ und $\phi_{\infty}=-42.26^{\circ}$. Im Folgenden sollen zeitlich und spannweitig gemittelte Grenzschichtprofile aus Hitzdrahtmessungen mit entsprechenden Profilen aus dieser Grenzschichtlösung verglichen werden. Damit soll gezeigt werden, dass Erkenntnisse aus der numerischen Grenzschichtlösung die Interpretation experimenteller Ergebnisse unterstützen können.

Das Grenzschichtströmungsfeld wurde nach dem in Abschnitt 5.1.4 beschriebenen Messablauf dreidimensional vermessen. Dabei wurden an Plattentiefenpositionen zwischen $x_{c} / c=0.1$ und $x_{c} / c=0.9$ im Abstand von $\Delta x_{c} / c=0.05$ spannweitig und wandnormal verteilte Hitzdrahtmessungen durchgeführt. Der 
(a) $x_{c} / c=0.2$

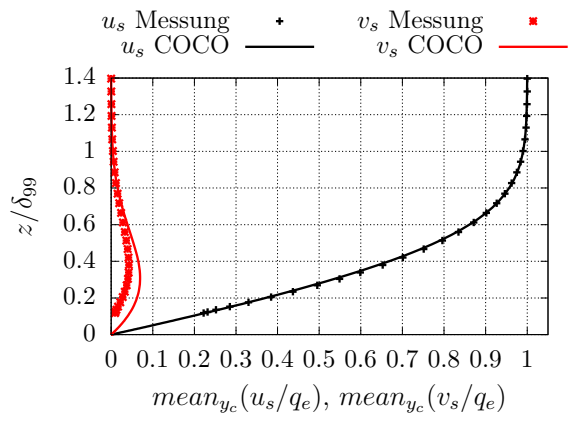

(c) $x_{c} / c=0.4$

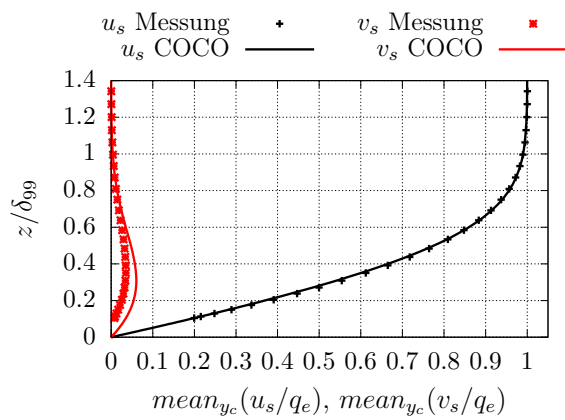

(b) $x_{c} / c=0.3$

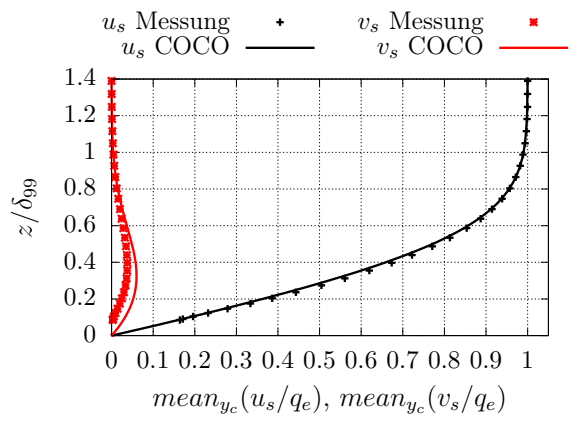

(d) $x_{c} / c=0.5$

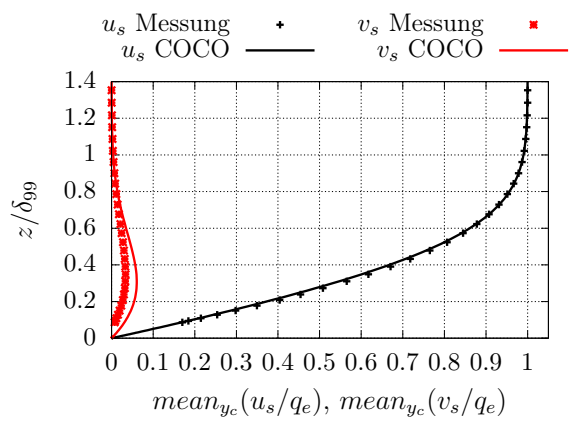

Abbildung 6.1.4: Vergleich der zeitlich und spannweitig gemittelten experimentellen $u_{s^{-}}$und $v_{s}$-Profile mit entsprechenden Profilen aus der numerischen COCOGrenzschichtlösung

Vergleich der experimentellen und numerischen Grenzschichtgrundströmung ist in Abbildung 6.1.4 anhand der $u_{s^{-}}$und $v_{s^{-}}$Komponente im Profiltiefenbereich $0.2 \leq x_{c} / c \leq 0.5$ dargestellt. Die wandnormale Koordinate $z$ wurde mit der lokalen Grenzschichtdicke $\delta_{99}$ entdimensionalisiert. Für die $u_{s}$-Komponente ergibt sich in diesem Profiltiefenbereich eine sehr gute Übereinstimmung zwischen dem Experiment und der numerischen Grenzschichtlösung. Die experimentell bestimmte und spannweitig gemittelte $v_{s}$-Komponente weist aber an jeder Profiltiefenposition einen geringeren Maximalwert auf als das numerische $v_{s}$-Profil.

Schon im ursprünglichen Prinzipexperiment der schiebenden ebenen Platte wurden Abweichungen zwischen den experimentellen und numerischen 
Grenzschichtprofilen besonders in der $v_{s}$-Komponente beobachtet [76]. Eine mögliche Erklärung ist die starke Abhängigkeit der experimentell bestimmten $v_{s}$-Komponente vom Rollwinkel der Hitzdraht-V-Sonde, wie in Abschnitt 5.1.6 näher beschrieben wurde. Insgesamt sind die Abweichungen der experimentellen Grenzschichtprofile von den numerischen Grenzschichtprofilen aber gering genug, um im Folgenden anzunehmen, dass Erkenntnisse aus der numerischen Grenzschichtströmung auf das Experiment übertragen werden können. Insbesondere wird im Folgenden die numerische Grenzschichtgrundströmung auf Stabilität untersucht und die Ergebnisse werden zur Interpretation der experimentellen Störströmung angewandt.

\subsection{Lineare Stabilitätseigenschaften}

Im vorangegangenen Abschnitt wurde gezeigt, dass die Grenzschichtströmung an der Modellplattenoberseite die im Rahmen der Neuauslegung des Prinzipexperiments gesteckten Ziele erfüllt. Die gemessene Druckverteilung und Grenzschichtrandgeschwindigkeit bestätigen die gewünschte starke Beschleunigung im vorderen Bereich der Modellplatte und schwächere Beschleunigung im Bereich stromab davon. Des Weiteren wurde gezeigt, dass eine numerisch bestimmte Grenzschichtlösung die experimentelle Grenzschichtströmung gut reproduzieren kann. Um die experimentell untersuchte Störströmung nachvollziehen und interpretieren zu können, soll nun in diesem Abschnitt diese Grenzschichtlösung linear und lokal auf Stabilität untersucht werden. Dabei soll im Folgenden vor allem geprüft werden, ob die Stabilitätseigenschaften der realisierten Grenzschichtströmung ebenfalls die Ziele der Neuauslegung erfüllen, siehe Abschnitt 4.2.

\subsubsection{Stationäre primäre Instabilitäten}

Die Grenzschichtlösung wurde mithilfe der Software NOLOT linear und lokal auf Stabilität bzgl. stationärer QSI-Moden untersucht. Wie in Abschnitt 2.3 beschrieben wurde, entspricht der N-Faktor einer Mode dem Integral ihrer Anfachungsrate. An einer $x_{c}$-Position drückt er aus, auf welche Amplitude $A$ eine anfängliche Amplitude $A_{0}$ dieser Mode stromab vom Neutralpunkt bis zu dieser Position von der instabilen Grenzschicht integral angefacht wurde: $A=A_{0} \cdot \exp (N)$. Die Mode, welche an einer $x_{c}$-Position den höchsten NFaktor erreicht, ist die integral angefachteste Mode an dieser Position. 
(a)

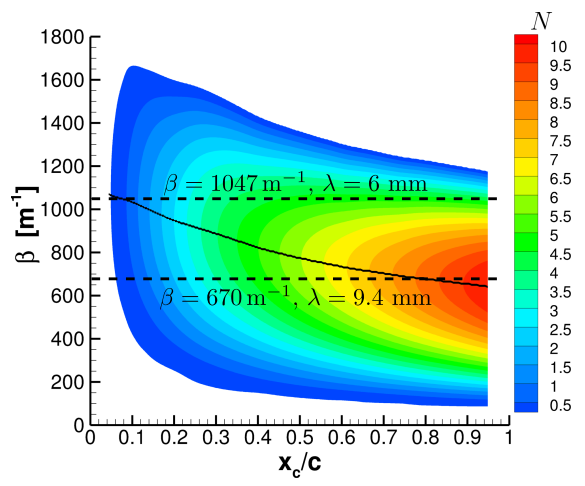

(b)

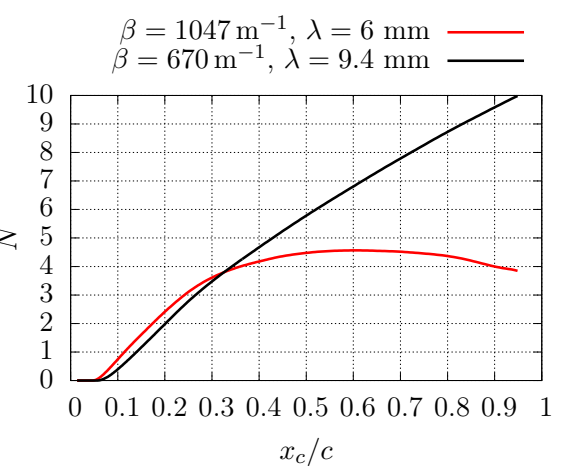

Abbildung 6.2.1: (a) N-Faktoren stationärer QSI-Moden in Isokonturen, abhängig von ihrer spannweitigen Wellenzahl $\beta$ und der Plattentiefenkoordinate $x_{c} / c$ gemäß linearer lokaler Stabilitätsuntersuchung (b) Vergleich des N-Faktor-Verlaufs der ausgewählten Kontrollmode und der natürlich dominanten stationären QSI-Mode

Die gemäß LST erreichten N-Faktoren der untersuchten stationären QSIModen sind in Abbildung 6.2.1(a) als Isokonturen abhängig von ihrer spannweitigen Wellenzahl $\beta$ und der Plattentiefenkoordinate $x_{c} / c$ dargestellt. Eine Linie verbindet die Wellenzahlen der jeweils integral angefachtesten Moden. Im Gegensatz zu den Stabilitätseigenschaften im ursprünglichen Prinzipexperiment (siehe Abschnitt 4.1) nimmt diese Wellenzahl monoton mit $x_{c}$ ab. Die Mode, welche an der letzten untersuchten Profiltiefenposition den höchsten N-Faktor erreicht, hat eine spannweitige Wellenzahl von $\beta=640 \mathrm{~m}^{-1}$. Man nennt sie die insgesamt integral angefachteste Mode. Abweichend von den LST-Ergebnissen der Neuauslegung besitzt die Mode, welche am weitesten stromauf den kritischen N-Faktor $N_{\text {crit }}=9$ erreicht, eine spannweitige Wellenzahl von $\beta=670 \mathrm{~m}^{-1}$ und eine spannweitige Wellenlänge von $\lambda=9.4 \mathrm{~mm}$ (vergleiche Abschnitt 4.3). Die Wellenzahl dieser als natürlich dominant erwarteten Mode ist in der Abbildung mit einer gestrichelten Linie markiert, wie auch die Wellenzahl $\beta=1047 \mathrm{~m}^{-1}$ der bei der Auslegung festgelegten Kontrollmode.

Die spannweitige Wellenlänge der natürlich dominanten Mode ist im Experiment etwas größer als während der Auslegung angenommen. Diese Abweichung ist dadurch begründet, dass die Anströmgeschwindigkeit für das Experiment geringer gewählt wurde als bei der Auslegung geplant war. Dadurch wurde erreicht, dass der finale Umschlag weder zu weit stromauf noch 
zu weit stromab stattfand. In Abschnitt 6.3.1 wird gezeigt, dass im Experiment als spannweitige Wellenlänge der tatsächlich dominanten stationären QSI-Mode ein noch etwas größerer Wert beobachtet wird. Damit entspricht das Verhältnis der spannweitigen Wellenlängen zwischen stationärer QSI-Kontrollmode und natürlich dominanter Mode nicht exakt zwei Dritteln wie bei Saric et al. [95]. Dies ist aber für die Funktionsweise der UFD-Methode nicht erforderlich, solange die in Abschnitt 4.2 geschilderten Ziele der Auslegung erfüllt sind (siehe auch Abschnitt 2.4.1).

In Abbildung 6.2.1(b) wird der N-Faktor-Verlauf dieser Kontrollmode und der natürlich dominanten stationären Instabilitätsmode im Vergleich gezeigt. Der Neutralpunkt der ausgewählten Kontrollmode (siehe auch Abschnitt 2.2), also die Plattentiefenposition, stromab von welcher sie nach linearer Theorie angefacht ist, liegt bei $x_{c} / c=0.05$. Die ausgewählte Kontrollmode erreicht im vorderen Bereich der Grenzschicht $0.05<x_{c} / c<0.35$ einen höheren N-Faktor als die natürlich dominante stationäre Instabilität, welche am wahrscheinlichsten für den laminar-turbulenten Umschlag verantwortlich ist. Somit kann bei geeigneter Anfangsamplitude die Kontrollmode an ausreichend stromauf gelegener Position hohe Amplituden erreichen, um nichtlinear eine Deformierung des spannweitig gemittelten Profils zu bewirken und dadurch die Grenzschicht zu stabilisieren, siehe Abschnitt 2.4. Daher kann also bestätigt werden, dass die Ziele der Auslegung (siehe Abschnitt 4.2) bzgl. der linearen Stabilitätseigenschaften gegenüber stationärer Instabilitätsmoden erreicht werden konnten. Mithilfe eines pneumatischen Aktuators der TU Berlin konnte bereits experimentell nachgewiesen werden, dass in der realisierten Grenzschichtströmung im neu ausgelegten Prinzipexperiment eine Transitionsverzögerung mithilfe der UFD-Methode möglich ist [68, 70]. In diesen Experimenten wurde allerdings die natürlich dominante stationäre QSI-Mode mithilfe von spannweitig periodischen DRE auf eine spannweitige Wellenlänge von $\lambda=9 \mathrm{~mm}$ fixiert, worauf im Rahmen dieser Arbeit verzichtet wurde.

\subsubsection{Instationäre primäre Instabilitäten}

Die Grenzschichtlösung wurde auch auf Stabilität bzgl. instationärer Querströmungsinstabilitäten untersucht. Für jede untersuchte Frequenz $f$ wurde dafür die Stabilität bzgl. einer Schar von QSI-Moden mit unterschiedlicher spannweitiger Wellenzahl $\beta$ untersucht, analog zur Diskussion im vorhergehenden Abschnitt für stationäre Moden, also für $f=0 \mathrm{~Hz}$. Durch rasterförmige Hitz- 
(a)

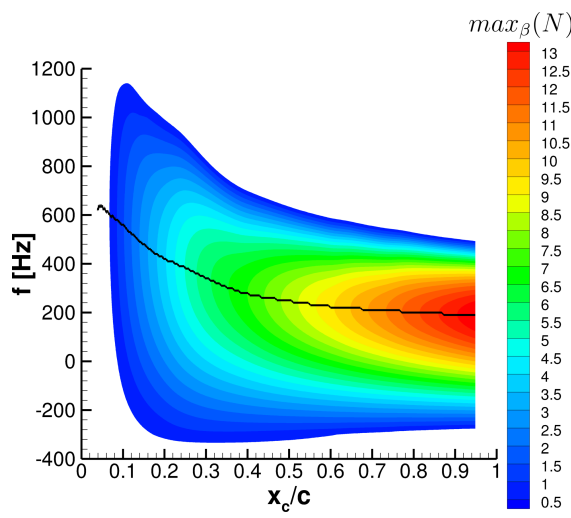

(b)

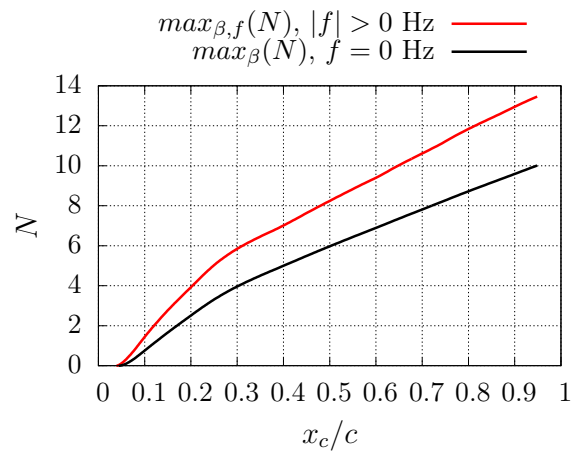

Abbildung 6.2.2: (a) Die bzgl. $\beta$ maximalen N-Faktoren instationärer QSI-Moden gemäß LST-Untersuchung (b) Vergleich der bzgl. $\beta$ und $f$ maximalen N-Faktoren instationärer $(|f|>0 \mathrm{~Hz})$ und der bzgl. $\beta$ maximalen N-Faktoren stationärer QSIModen $(f=0 \mathrm{~Hz})$

drahtmessungen mit unbekanntem Phasenbezug zur instationären Anregung können keine Informationen über das Wellenzahlspektrum der beobachteten instationären Störungen in der Grenzschicht gewonnen werden. Um zu diskutieren, in welchem Frequenzbereich laufende QSI im Experiment erwartet werden können, sofern eine geeignete Anregung in der Anströmung enthalten ist, ist in Abbildung 6.2.2(a) der an jeder Plattentiefenposition $x_{c} / c$ bzgl. $\beta$ maximale N-Faktor abhängig von der Frequenz $f$ und $x_{c} / c$ zu sehen. Dabei erstreckt sich das Diagramm auch in den Bereich negativer Frequenzen. Es sind neben Moden mit positiven Werten von $\beta$ auch Moden mit negativen Werten von $\beta$ angefacht. In Abschnitt 2.2 wurde der Störansatz 2.2.7 eingeführt. Er enthält stets auch das komplex Konjugierte des ersten Termes. Daher beschreibt eine Mode mit $-f$ und $\beta$ dieselbe physikalisch relevante Lösung der Stördifferentialgleichungen wie eine Mode mit $f$ und $-\beta$.

Mit einer schwarzen Linie wurden in der Abbildung die Frequenzen der Moden mit dem jeweils maximalen N-Faktor an jeder Position verbunden. Dies zeigt, dass die Frequenz der maximal integral angefachten laufenden QSI mit zunehmender Plattentiefenposition abnimmt, bis sie den Wert der insgesamt integral angefachtesten Moden mit $f=190 \mathrm{~Hz}$ erreicht. Des Weiteren ist zu erkennen, dass laufende Primärinstabilitäten oberhalb von $f=1000 \mathrm{~Hz}$ nur geringe N-Faktoren erreichen und auch nur in einem kleinen Plattentiefen- 
bereich angefacht sind. Die bzgl. $\beta$ und $f$ maximalen N-Faktoren laufender QSI sind auch in Abbildung 6.2.2(b) im Vergleich zu den bzgl. $\beta$ maximalen N-Faktoren stationärer QSI dargestellt. Dabei ist zu erkennen, dass laufende QSI wie erwartet im gesamten Verlauf der Grenzschicht stärker integral angefacht sind als stationäre, siehe auch Abschnitt 2.3. Welche Instabilitätsform im Experiment das Transitionsszenario dominiert, hängt aber zusätzlich vom jeweiligen Anregungsniveau und der Rezeptivität der Grenzschicht ab.

\subsection{Transitionsszenario im neu ausgelegten Prinzipexperiment}

Nachdem in den vorigen Abschnitten die Ziele der Neuauslegung des Prinzipexperiments experimentell bestätigt wurden und die numerische Grenzschichtgrundströmung auf Stabilität untersucht wurde, wird in diesem Abschnitt das Transitionsszenario im neu ausgelegten Prinzipexperiment inklusive der natürlich auftretenden Störströmung experimentell untersucht. Es wird vor allem geprüft, ob die beobachtete Störströmung anhand der Stabilitätsergebnisse nachvollzogen werden kann. Dabei wurde experimentell weiterhin der Naseneinsatz mit Druckbohrungen verwendet und keine künstliche Anregung stationärer QSI (z.B. durch DRE) eingesetzt, im Gegensatz zu vielen anderen experimentellen Studien zu querströmungsdominierter Transition (z.B. [68, 70, 116]). Die genaue Kenntnis des Transitionsszenarios in diesem Referenzfall ermöglicht in den folgenden Kapiteln die Interpretation des Effekts der Aktuierung auf das Transitionsszenario.

\subsubsection{Stationäre Störströmung}

Wie in Abschnitt 2.3 beschrieben, bewirken stationäre QSI eine spannweitig periodische Deformation des zeitlich gemittelten Strömungsfeldes in der Grenzschicht, während die Grenzschichtgrundströmung idealisiert spannweitig invariant ist. Jeder der spannweitig periodisch angeordneten, gleichsinnig rotierenden Längswirbel transportiert an einer spannweitigen Flanke impulsarmes Fluid aus Wandnähe in höhere Schichten und an seiner anderen spannweitigen Flanke impulsreiches Fluid zur Wand hin. Daher sind stationäre QSI in ihrem Verlauf in der Grenzschicht durch ihre spannweitig periodische lokale Deformation der zeitlich gemittelten Grenzschichtprofile zu erkennen. Dazu ist die zeitlich gemittelte $u_{s}$-Komponente aus spannweitig und wandnormal verteilten rasterförmigen Grenzschichtmessungen in Abbildung 


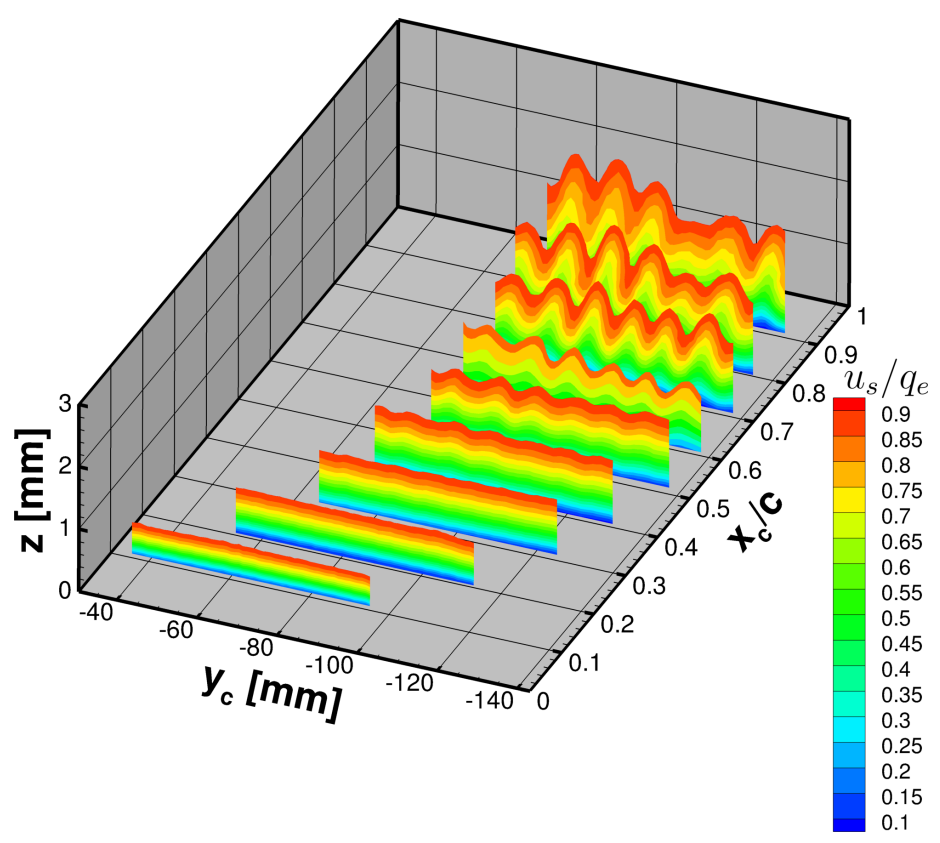

Abbildung 6.3.1: Deformation der zeitlich gemittelten $u_{s}$-Grenzschichtprofile aus rasterförmigen Hitzdrahtmessungen

6.3.1 an ausgewählten $x_{c}$-Positionen dargestellt. Dabei ist etwa bis $x_{c} / c=0.4$ kaum lokale Deformation erkennbar, die Amplitude der stationären QSI ist noch gering. Ab $x_{c} / c=0.4$ ist eine deutliche lokale Deformation der $u_{s}$-Profile durch stationäre QSI erkennbar. Einzelne Längswirbel mit leicht unterschiedlicher Amplitude können stromab verfolgt werden und das Amplitudenwachstum stationärer QSI wird durch die in $x_{c}$-Richtung zunehmende Deformation deutlich. Bei der Wahl des jeweiligen spannweitigen Messbereichs wurde versucht, dieselben Längswirbel stromab zu verfolgen, was nicht immer vollständig gelungen ist. Bei ausreichend gut erfüllten unendlich schiebenden Bedingungen und spannweitig homogener Anregung stationärer QSI sollte der spannweitige Messbereich aber repräsentativ für die spannweitig verteilte Situation sein.

Auch der einsetzende finale Umschlag ist in dieser Darstellung zwischen $x_{c} / c=0.7$ und $x_{c} / c=0.9$ zu erkennen. Dort nimmt die lokale Deformation durch einzelne Längswirbel wieder ab, was durch den turbulenten Zerfall dieser Längswirbel begründet ist. Wie in Abschnitt 6.3.3 beschrieben, entsteht 
nichtlinear eine Deformation des spannweitig gemittelten Grenzschichtprofils und die spannweitig mittlere Grenzschichtdicke $\delta_{99}$ nimmt stärker zu als die einer laminaren Grenzschicht. Dies ist allerdings in dieser dreidimensionalen Darstellung nicht offensichtlich.

Bei $x_{c} / c=0.6$ sind die zeitlich gemittelten $u_{s}$-Profile spannweitig lokal signifikant deformiert, während das spannweitig und zeitlich gemittelte Profil in Abbildung 6.3.4 noch nahezu dem der numerischen Grundströmung entspricht. An dieser Position soll im Folgenden das spannweitige Spektrum stationärer QSI mit dem Spektrum der integralen Anfachung gemäß LST (siehe Abschnitt 6.2) verglichen werden. Betrachtet man die vermessene Ebene bei $x_{c} / c=0.6$, sind ungefähr sechs Perioden auf einer spannweitigen Strecke von $\sim 60 \mathrm{~mm}$ zu beobachten. Dadurch lässt sich die spannweitige Wellenlänge der dominanten stationären QSI grob auf $\lambda \approx 10 \mathrm{~mm}$ abschätzen, was $\beta=\frac{2 \pi}{\lambda}=628 \mathrm{~m}^{-1}$ entspricht und somit näherungsweise zu der Wellenzahl der in Abschnitt 6.2.1 beschriebenen insgesamt integral angefachtesten stationären QSI-Mode passt. Die Tatsache, dass einzelne Längswirbel mit leicht unterschiedlicher Amplitude auftreten, weist darauf hin, dass das stationäre Störungsspektrum neben dieser Mode weitere Anteile mit anderen spannweitigen Wellenzahlen enthält. Mit dem Ende des linearen Bereichs kann die spannweitige Deformation der stationären Grenzschichtprofile stromab nicht mehr rein als Folge primärer QSI interpretiert werden.

Um quantitative Informationen über die Zusammensetzung des Wellenzahlspektrums stationärer QSI zu erhalten, wurde die spannweitig periodische stationäre Grenzschichtdeformation spektral untersucht. Dazu wurde zu jeder Profiltiefe und für jeden untersuchten Wandabstand der spannweitige Verlauf $u_{s}\left(y_{c}\right)$ mithilfe einer diskreten Fourier-Transformation spektral in harmonische Anteile mit spannweitiger Wellenzahl $\beta$ zerlegt. Dazu wurde eine Implementierung der Fast Fourier Transformation aus der SoftwareBibliothek NumPy und eine Von-Hann-Fensterfunktion verwendet. Die dabei bestimmten komplexwertigen Fourierkoeffizienten $\breve{u}_{s}(\beta)$ sind die Amplituden der harmonischen Anteile $u_{s, \beta}^{*}$, welche sich wie folgt darstellen lassen:

$$
\begin{aligned}
u_{s, \beta}^{*} & =\check{u}_{s}(\beta) e^{i \beta y_{c}} \\
& =\hat{u}_{s}(\beta) e^{i \varphi(\beta)} e^{i \beta y_{c}} \\
& =\hat{u}_{s}(\beta) e^{i\left(\beta y_{c}+\varphi(\beta)\right)}
\end{aligned}
$$

Dabei wurde der Fourierkoeffizient $\check{u}_{s}(\beta)$ durch seinen reellwertigen Betrag

$$
\hat{u}_{s}=\left|\check{u}_{s}\right|=\sqrt{\Re^{2}\left(\check{u}_{s}\right)+\Im^{2}\left(\check{u}_{s}\right)}
$$


und seine Phase $\varphi$ ausgedrückt. Im Folgenden wird $\hat{u}_{s}(\beta)$ als die Amplitude der spannweitigen $u_{s}$-Periodizität mit der spannweitigen Wellenzahl $\beta$ und $\varphi(\beta)$ als ihre Phasenlage bezeichnet. Die spektrale Auflösung bzgl. $\beta$ wird durch die spannweitige Abtastrate $f_{s}$ und die Anzahl der abgetasteten Werte $Z$ bestimmt:

$$
\Delta \beta=\frac{2 \pi f_{s}}{Z}
$$

Dabei entspricht die Abtastrate $f_{s}=1 / \Delta y_{c}$ mit der spannweitigen Schrittweite $\Delta y_{c}=0.99 \mathrm{~mm}$. Die Anzahl der spannweitig verteilten Messwerte beträgt für spannweitig und wandnormal verteilte Flächenmessungen $Z=61$, siehe auch Abschnitt 5.1.4. Da die spannweitige Länge des Messbereichs $L=(Z-1) \cdot \Delta y_{c}$ entspricht, gilt auch:

$$
\Delta \beta=\frac{2 \pi}{Z \Delta y_{c}}=\frac{2 \pi}{L+\Delta y_{c}}=104.05 \mathrm{~m}^{-1}
$$

Solange also die spannweitige Schrittweite $\Delta y_{c}$ klein im Vergleich zu $L$ ist, wird die Auflösung $\Delta \beta$ maßgeblich durch die Länge des spannweitigen Messbereichs $L$ (hier $L=59.4 \mathrm{~mm}$ ) bestimmt.

Das wandnormale Maximum der auf diese Art gewonnenen spektralen Instabilitätsamplitude $\hat{u}_{s}$ ist als Isokonturendiagramm in Abbildung 6.3.2(a) abhängig von der Plattentiefenkoordinate $x_{c} / c$ und der spannweitigen Wellenzahl $\beta$ dargestellt. Sie wird meist auf die jeweilige Grenzschichtrandgeschwindigkeit $q_{e}\left(x_{c}\right)$ bezogen. Die mit der LST-Untersuchung erwartete integrale Anfachung stationärer Moden wurde in Abschnitt 6.2.1 als N-Faktor abhängig von $x_{c} / c$ und $\beta$ dargestellt. Wählt man eine Anfangsamplitude $\hat{u}_{s, 0}$, lassen sich die gemessenen Amplituden $\hat{u}_{s} / q_{e}$ mit den gemäß LST erwarteten Amplituden $\hat{u}_{s, L S T} / q_{e}=\hat{u}_{s, 0} / q_{e} \cdot e^{N}$ vergleichen. Diese erwarteten Amplituden sind als Isolinien in Abbildung 6.3.2(a) eingezeichnet, wobei ihr Wert auf der jeweiligen Isolinie markiert wurde.

Es wird deutlich, dass gemäß LST ein breiteres Spektrum erwartet wird als man im Experiment beobachtet. Stattdessen dominiert die stationäre Instabilitätsmode bei $\beta=624 \mathrm{~m}^{-1}$ schon früh das experimentelle Transitionsszenario. An der Plattentiefenposition ihres Amplitudenmaximums ist ihre spannweitige Wellenzahl geringfügig kleiner als diejenige der dort integral am stärksten angefachten Mode. Dies könnte ein Hinweis darauf sein, dass das spannweitige Spektrum der relevanten Anregung für stationäre QSI nicht homogen bzgl. $\beta$ verteilt ist, sondern ein erhöhter Anteil bei dieser Wellenzahl enthalten ist. 
(a)

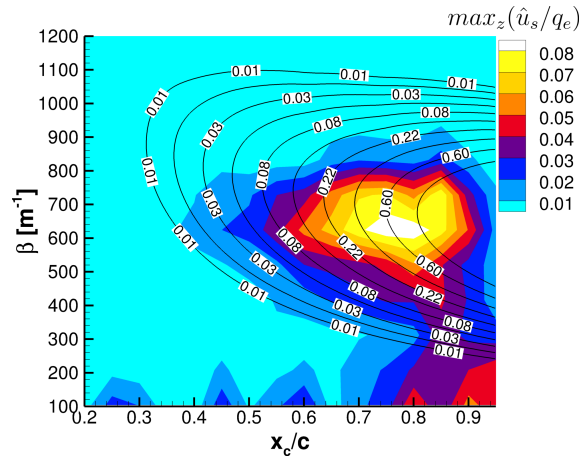

(b)

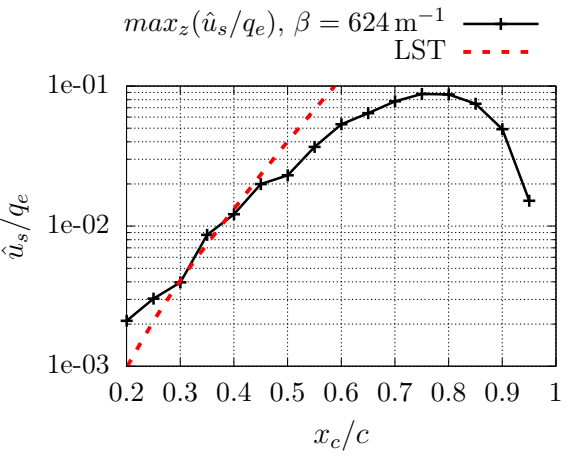

Abbildung 6.3.2: (a) Wandnormal maximale Amplitude $\hat{u}_{s}$ stationärer QSI im Vergleich mit der gemäß LST erwarteten Amplitude $\hat{u}_{s, L S T} / q_{e}$ als Isolinien (b) Räumliches Wachstum der wandnormal maximalen Amplitude bei $\beta=624 \mathrm{~m}^{-1} \mathrm{im}$ Vergleich mit der gemäß LST erwarteten Amplitude $\hat{u}_{s, L S T} / q_{e}$

Die relevante Anregung für stationäre QSI ist die Oberflächentopologie des untersuchten Naseneinsatzes. Seine Oberfläche wurde durch manuelles Schleifen bearbeitet, sodass vor den Windkanalexperimenten angenommen wurde, dass die verteilte Rauigkeit ein breit verteiltes Spektrum besitzt. Das räumliche Spektrum dieses Naseneinsatzes wurde daher nicht untersucht. Allerdings wurde bei einer Vermessung der Oberflächentopologie eines gleichartig gefertigten Naseneinsatzes für ein anderes Projekt ein lokales Maximum um $\lambda=10 \mathrm{~mm}$ im spannweitigen Spektrum festgestellt [60]. Eine inverse Fourier-Transformation des spannweitigen Spektrums der Oberflächentopologie offenbarte regelmäßige Strukturen parallel zur $x_{c}$-Achse mit dieser spannweitigen Wellenlänge, welche möglicherweise durch den Werkzeugpfad der CNC-Fräse bei der Herstellung verursacht wurden [60]. Womöglich waren solche Strukturen auch in der Oberflächentopologie des hier untersuchten Naseneinsatzes vorhanden und führten zu einer verstärkten Anregung bei dieser Wellenlänge. Da die Anfachung der Grenzschicht spektral nicht sehr selektiv bzgl. $\beta$ bzw. $\lambda$ ist, kann schon eine geringfügig angehobene Anregung bei dieser spannweitigen Wellenlänge diese frühe Dominanz erklären. Ein nachträglicher Nachweis einer solchen diskreten Anregung war leider nicht möglich, da die Oberfläche des Naseneinsatzes für ein nachfolgendes Projekt auf ein sehr geringes Rauigkeitsniveau geschliffen wurde. 
In Abbildung 6.3.2(a) fallen bei hohen $x_{c}$-Werten langwellige spektrale Anteile auf. Wie schon zu Abb. 6.3.1 beschrieben wurde, zeigt sich der beginnende finale Umschlag zwischen $x_{c} / c=0.8$ und $x_{c} / c=0.9$ unter anderem am Zerfall der stationären Wirbel und der Abnahme ihrer spannweitig periodischen Deformation der Grenzschicht. Dieser Zerfall findet nicht spannweitig homogen statt, sondern einzelne Längswirbel zerfallen an voneinander verschiedenen $x_{c}$-Positionen. Dies führt dazu, dass im spannweitigen Spektrum der stationären Grenzschichtdeformation langwellige Anteile für $\beta<400 \mathrm{~m}^{-1}$ beobachtet werden.

Die wandnormal maximale Amplitude $\hat{u}_{s}$ der QSI-Mode mit $\beta=624 \mathrm{~m}^{-1}$ ist in Abbildung 6.3.2(b) logarithmisch dargestellt. In dieser Darstellung hat das Wachstum stromauf von $x_{c} / c=0.6$ nahezu einen linearen Charakter, was auf ein exponentielles Wachstum hinweist. Dieses Wachstum verläuft stromauf von $x_{c} / c=0.45 \mathrm{im}$ Wesentlichen parallel zur ebenfalls eingezeichneten integralen Anfachung der Mode gemäß LST $\hat{u}_{s, L S T} / q_{e}$. Stromab von $x_{c} / c=0.45$ wird ein deutlich schwächeres Wachstum der Amplitude beobachtet. Sie erreicht einen Maximalwert von $\hat{u}_{s} / q_{e}=0.09$ bei $x_{c} / c=0.75$. In Abschnitt 2.3 wurde dies bereits als „nichtlineare Sättigung“ und der Maximalwert als Sättigungsamplitude beschrieben. Im ursprünglichen Prinzipexperiment wurde als Sättigungsamplitude der dominanten QSI-Mode ein nahezu gleicher Wert beobachtet $[22,76]$. Dies weist darauf hin, dass ein ähnliches Verhältnis der relevanten Anregungsniveaus von stationären und laufenden QSI vorliegt. Das schwächere Wachstum und das Erreichen eines Sättigungswertes sind nichtlineare Phänomene im fortgeschrittenen Stadium der Transition. In Abschnitt 6.3.3 werden die spannweitig gemittelten Grenzschichtprofile stromab von $x_{c} / c=0.6$ mit der numerischen Grundströmung verglichen. Dabei wird auf diese Beobachtung Bezug genommen.

Zusammenfassend wird im Experiment als natürlich dominante stationäre QSI-Mode diejenige mit der spannweitigen Wellenzahl $\beta=624 \mathrm{~m}^{-1}$ beobachtet. Diese liegt im Wellenzahlbereich der gemäß LST insgesamt am stärksten integral angefachten stationären QSI. Das beobachtete stationäre Störspektrum lässt sich also mit den Ergebnissen der LST-Untersuchung im Wesentlichen nachvollziehen. Im Bereich kleiner Amplituden gilt dies auch für die Amplitudenentwicklung der natürlich dominanten Mode. 


\subsubsection{Instationäre Störströmung}

Da laufende QSI die Grenzschichtprofile anders als stationäre QSI nicht spannweitig ortsfest deformieren, kann ihr $\beta$-Spektrum mithilfe einer einzelnen Hitzdrahtsonde ohne festen Phasenbezug zwischen der Messung und der instationären Anregung nicht untersucht werden. Es ist im Bereich linearer Störungsentwicklung zu erwarten, dass laufende QSI in den rasterförmigen Messungen als spannweitig homogen verteilte instationäre Geschwindigkeitsfluktuationen in Erscheinung treten, siehe auch Abschnitt 2.3. Im Folgenden werden spannweitig gemittelte Amplituden instationärer $u_{s}$-Fluktuationen diskutiert, um zu untersuchen, welche spektralen Anteile durch Vergleich mit LST-Ergebnissen als Konsequenz laufender QSI-Moden identifiziert werden können.

In Abschnitt 5.2.1 wurde die instationäre Anströmung stromauf des Modells charakterisiert und die spektrale Verteilung $u_{A, 0}^{\prime}(f)$ instationärer Geschwindigkeitsfluktuationen in der Anströmung beschrieben. Treffen diese Fluktuationen auf die Grenzschicht an der Modellplatte, koppeln sie im Rahmen des Rezeptivitätsprozesses als Anfangsanregung einer laufenden QSI in die Grenzschicht ein. Diese Anfangsanregung kann dann in der instabilen Grenzschicht angefacht sein und bis zur jeweiligen Messposition in ihrer Amplitude anwachsen. Wegen der fehlenden Information über das Wellenzahl-Spektrum ist kein gezielter Vergleich einzelner laufender Moden mit ihrer linearen integralen Anfachung gemäß LST möglich. Stattdessen soll im Folgenden als obere Abschätzung für theoretisch zu erwartende Amplituden laufender Moden die bei jeder Frequenz $f$ maximale integrale Anfachung diskutiert werden. Im Experiment werden die beobachteten Fluktuationen bei einer Frequenz $f$ aber durch eine Überlagerung der beteiligten Moden mit verschiedenen Wellenzahlen verursacht, deren integrale Anfachung jeweils geringer ist als die der maximal integral angefachten Mode. Die obere Abschätzung für die Amplitude laufender QSI an der Position $x_{c}$ mit der Frequenz $f$ entspricht

$$
u_{A}^{\prime}\left(x_{c}, f\right)=u_{A, 0}^{\prime}(f) \cdot r_{K} \cdot e^{N_{\max }\left(f, x_{c}\right)},
$$

mit den in der LST-Untersuchung ermittelten bzgl. $\beta$ maximalen N-Faktoren $N_{\max }$ an der Position $x_{c}$ (siehe Abschnitt 6.2.2). Der konstant angenommene Koeffizient $r_{K}$ ist durch die unbekannte und im Rahmen der Arbeit nicht untersuchte Rezeptivität der Grenzschicht begründet. Er wird im Folgenden so gewählt, dass $u_{A}^{\prime}\left(x_{c}, f\right)$ an der Position $x_{c} / c=0.3$ in einem möglichst großen Frequenzbereich den experimentellen spannweitig gemittelten Amplituden instationärer Fluktuationen entspricht. 


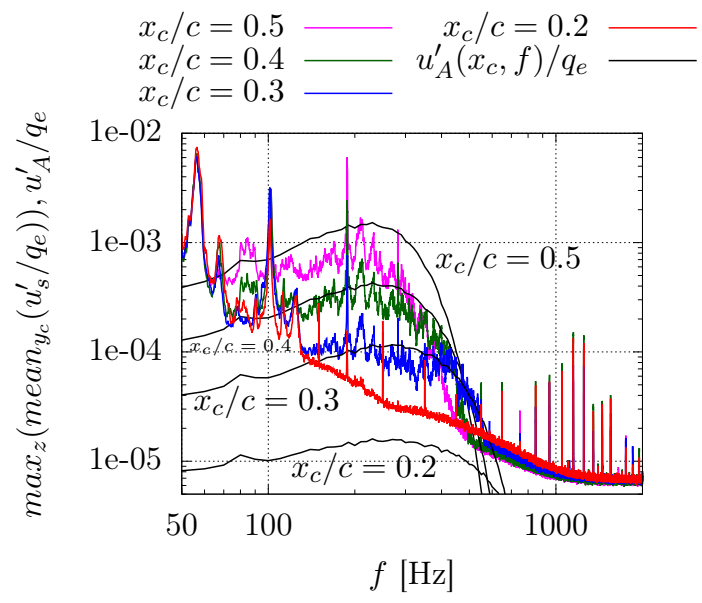

Abbildung 6.3.3: Spannweitig gemittelte und wandnormal maximale Amplituden instationärer Fluktuationen bei verschiedenen Plattentiefenpositionen in Vergleich mit den theoretischen Verteilungen maximal erwarteter Amplituden

Diese theoretische Verteilung maximal zu erwartender Amplituden berücksichtigt die spektrale Frequenzverteilung der instationären Störungen in der Anströmung und mit der beschriebenen Einschränkung die Frequenzabhängigkeit der selektiven linearen Anfachung der Grenzschicht. Die Verteilung ist zum Vergleich mit den experimentellen Spektren spannweitig gemittelter instationärer Fluktuationsamplituden für die Plattentiefenpositionen $0.2 \leq x_{c} / c \leq 0.5$ in Abbildung 6.3.3 eingezeichnet. Beim Vergleich der Verteilungen erkennt man, dass im Frequenzbereich $f<100 \mathrm{~Hz}$ spektrale Inhalte gemessen werden, die unabhängig von der Plattentiefenposition mit gleicher Stärke auftreten und daher nicht als Auswirkung angefachter instationärer Instabilitäten zu deuten sind. In Abschnitt 5.2.1 wurden in der Anströmung stromauf der experimentellen Konfiguration ebenfalls auffällige lokale Maxima im Spektrum instationärer Fluktuationen im Frequenzbereich $f<100 \mathrm{~Hz}$ beobachtet. Die hohen Amplituden in diesem Frequenzbereich werden im Folgenden nicht zur Diskussion laufender QSI herangezogen. Zudem sind im gesamten Spektrum diskrete scharfe lokale Maxima bei Vielfachen der Netzfrequenz $50 \mathrm{~Hz}$ zu erkennen.

Für den Frequenzbereich $100 \mathrm{~Hz}<f<600 \mathrm{~Hz}$ sind ab $x_{c} / c=0.3$ gegenüber $x_{c} / c=0.2$ erhöhte Amplituden zu erkennen, welche auch mit der Plattentiefe stromab weiter anwachsen. Ihre spektrale Verteilung bzgl. der Frequenz stimmt qualitativ gut mit der theoretischen Verteilung maximal erwarteter 
Amplituden $u_{A}^{\prime}\left(x_{c}, f\right)$ überein. Insbesondere lässt sich auch die obere Grenzfrequenz angeregter und integral angefachter laufender QSI nachvollziehen. Bei $x_{c} / c=0.3$ liegt sie bspw. knapp unter $f=600 \mathrm{~Hz}$. Sie ist eine Konsequenz aus der geringen integralen Anfachung von laufenden QSI bei höheren Frequenzen (siehe Abbildung 6.2.2(a)) und der Tatsache, dass schon im Spektrum der instationären Anströmung Fluktuationen mit niedriger Frequenz stärker vertreten sind als Fluktuationen hoher Frequenz (siehe Abbildung 5.2.3(b)). Diese Beobachtung motiviert, dass im Folgenden Effektivwerte von Geschwindigkeitsschwankungen im Frequenzbereich $100 \mathrm{~Hz}<f<600 \mathrm{~Hz}$ als Anzeichen laufender QSI interpretiert werden.

Angehobene Effektivwerte von Fluktuationen mit höheren Frequenzen, insbesondere mit Frequenzen $f>1000 \mathrm{~Hz}$ werden nur aufgrund von hochfrequenten Sekundärinstabilitäten oder aufgrund von turbulenten Fluktuationen erwartet. Bei der Interpretation hochfrequenter Geschwindigkeitsschwankungen müssen zusätzliche Indizien herangezogen werden, um zwischen hochfrequenter Sekundärinstabilität und Turbulenz unterscheiden zu können. Wie in Abschnitt 2.3 beschrieben, ist Sekundärinstabilität an die Grenzschichtdeformation durch die Primärinstabilitäten gebunden und bei stationären Primärinstabilitäten daher örtlich stark lokalisiert. Zusätzliche Indizien für den finalen Umschlag wären das Aufdicken der Grenzschichtdicke aufgrund des erhöhten wandnormalen Impulsaustausches und der Zerfall der stationären Längswirbel.

Gemäß der LST-Ergebnisse nimmt die obere Grenzfrequenz der integral angefachten laufenden QSI-Moden mit zunehmender Plattentiefe $x_{c}$ ab. Dies ist auch in den experimentellen Spektren in Abbildung 6.3.3 zu beobachten. Das Amplitudenwachstum entlang $x_{c}$ bleibt in den Messungen wie aufgrund obiger Diskussion erwartet hinter dem Wachstum der theoretisch erwarteten maximalen Amplituden zurück.

Es lässt sich zusammenfassend feststellen, dass ein Frequenzbereich identifiziert wurde, in dem die beobachteten instationären Fluktuationen mit angefachten laufenden QSI assoziiert werden können. Zudem wurde eine obere Grenzfrequenz laufender QSI beobachtet, oberhalb derer instationäre Fluktuationen mit großer Wahrscheinlichkeit eine andere Ursache haben. 


\subsubsection{Spannweitig und zeitlich gemittelte Grenzschichtströmung im fortgeschrittenen Stadium}

In Abschnitt 6.1.4 wurden bereits spannweitig und zeitlich gemittelte Grenzschichtprofile im Profiltiefenbereich $0.2 \leq x_{c} / c \leq 0.5$ mit Grenzschichtprofilen aus der numerischen Grenzschichtlösung verglichen. Dort wurde besonders in der $u_{s}$-Komponente eine gute Übereinstimmung beobachtet. Dies ist ein Hinweis darauf, dass die Amplituden der Störströmung ausreichend gering sind, dass diese der Grundströmung ohne einen signifikanten Einfluss überlagert ist.

In Abbildung 6.3.4 sind nun die spannweitig und zeitlich gemittelten Grenzschichtprofile für den Profiltiefenbereich $0.6 \leq x_{c} / c \leq 0.9 \mathrm{im}$ Vergleich zur numerischen Grundströmung dargestellt. Auch bei $x_{c} / c=0.6$ ist noch eine gute Übereinstimmung bis auf die bereits diskutierte Abweichung der $v_{s}$-Komponente zu beobachten. Ab $x_{c} / c=0.7$ fällt aber auch eine Abweichung des spannweitig gemittelten $u_{s}$-Profils vom Grundströmungsprofil auf. Für die Wandabstände $0.1<z / \delta_{99}<0.6$ sind die $u_{s}$-Werte erhöht, für $0.6<z / \delta_{99}<0.9$ sind sie leicht verringert. Diese Abweichung verstärkt sich stromab bei $x_{c} / c=0.8$ und $x_{c} / c=0.9$. Bei $x_{c} / c=0.7$ besitzt die dominante stationäre QSI-Mode mit $\beta=624 \mathrm{~m}^{-1}$ bereits eine Amplitude von $\hat{u}_{s} / q_{e}=0.08$ und erreicht bei $x_{c} / c=0.75$ ihre maximale Amplitude von $\hat{u}_{s} / q_{e}=0.09$. Die beobachtete Deformation der spannweitig und zeitlich gemittelten Grenzschichtströmung in diesem Profiltiefenbereich lässt sich durch eine nichtlineare Wechselwirkung der stationären QSI erklären (vgl. z.B. auch [76]). Eine solche Deformation ist typisch für die späte Phase des querströmungsdominierten Transitionsprozesses.

Aus den spannweitig gemittelten Geschwindigkeitsprofilen lassen sich an jeder Plattentiefenposition die Grenzschichtdicke $\delta_{99}$, die Verdrängungsdicke $\delta_{1}$ und die Impulsverlustdicke $\delta_{2}$ auswerten. Diese Grenzschichtparameter sind für den Referenzfall gemeinsam mit dem Formparameter $H_{12}=\frac{\delta_{1}}{\delta_{2}} \mathrm{im}$ Vergleich mit den entsprechenden Parametern aus der numerischen Grenzschichtgrundströmung in Abbildung 6.3.5 dargestellt. Die experimentell bestimmten Verläufe von $\delta_{99}, \delta_{1}$ und $\delta_{2}$ weisen über einen großen Teil der Profiltiefe eine gute Übereinstimmung mit den jeweiligen Verläufen aus der numerischen Grundströmung auf. Man erkennt bereits am stärkeren Anwachsen der Grenzschichtdicke $\delta_{99}$ stromab von $x_{c} / c=0.7 \mathrm{im}$ Vergleich zum Verlauf der numerischen Grundströmung den Beginn des finalen Umschlags 

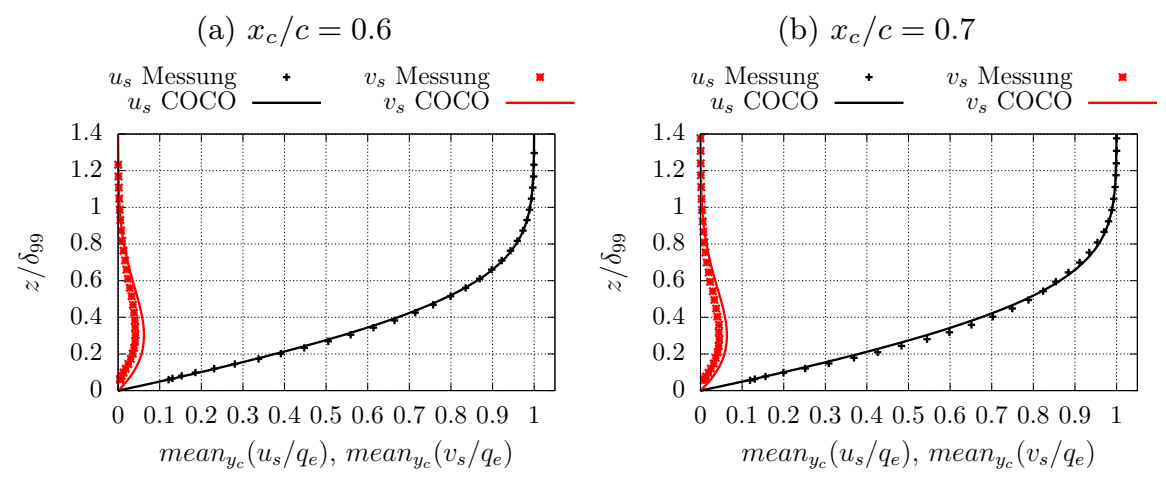

(c) $x_{c} / c=0.8$

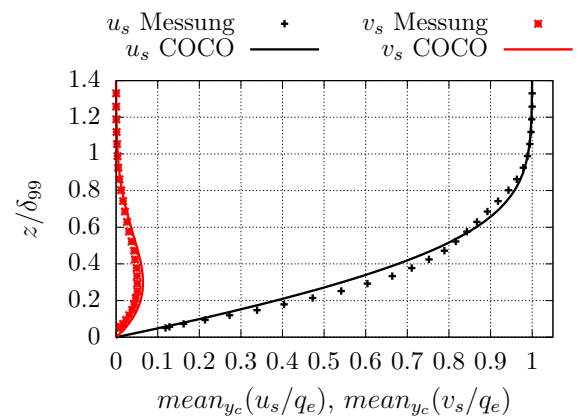

(d) $x_{c} / c=0.9$

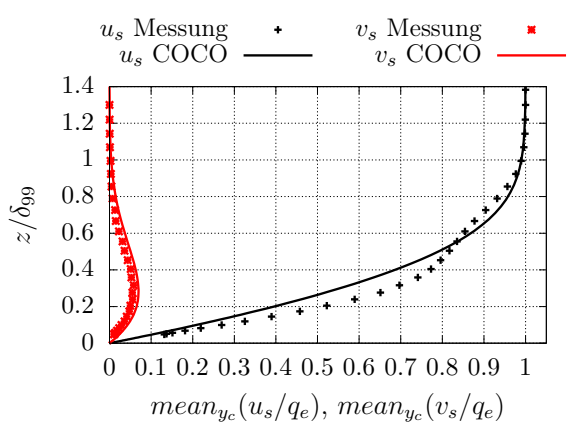

Abbildung 6.3.4: Spannweitig gemittelte wandnormale Profile der zeitlich gemittelten Geschwindigkeitskomponenten $u_{s}$ und $v_{s}$ im Vergleich zu den Ergebnissen der numerischen Strömungssimulation mit TAU (siehe Abschnitte 3.1 und 6.1)

im Experiment. Auch der Formparameter $H_{12}$ zeigt stromauf von $x_{c} / c=0.6$ eine gute Übereinstimmung mit dem numerisch bestimmten Formparameter. Die stärkere Abnahme seines Wertes relativ zum numerischen Vergleichswert zwischen $x_{c} / c=0.6$ und $x_{c} / c=0.9$ deutet auch auf die fülliger werdende Form des zeitlich und spannweitig gemittelten Grenzschichtprofils aufgrund des einsetzenden finalen Umschlags hin. Besonders bei $x_{c} / c=0.9$ ist deutlich, dass der wandnormale Gradient der $u_{s}$-Komponente in Wandnähe deutlich größer ist als in der laminaren Grundströmung. Dies entspricht wie für den laminar-turbulenten Umschlag typisch einer erhöhten Wandschubspannung. 


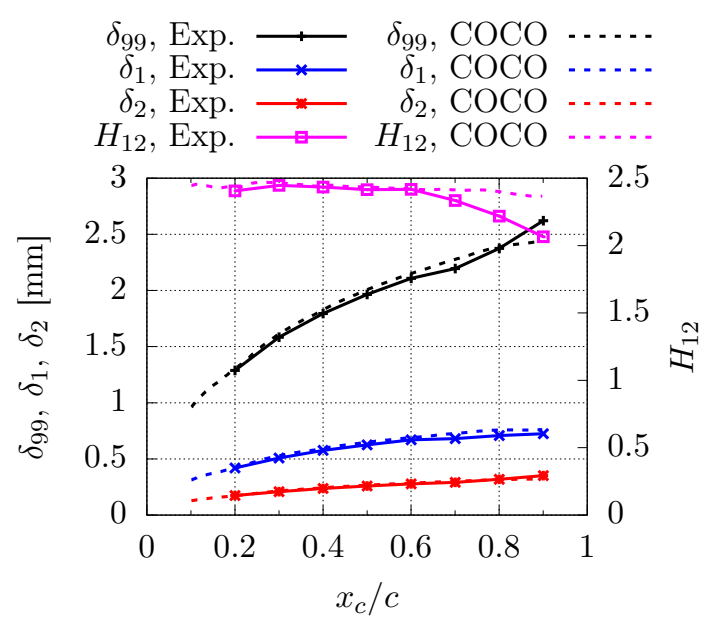

Abbildung 6.3.5: Entwicklung der Grenzschichtdicke $\delta_{99}$, der Verdrängungsdicke $\delta_{1}$, der Impulsverlustdicke $\delta_{2}$ sowie des Formparameters $H_{12}$ über die Plattentiefe $x_{c} / c$

Stromab von $x_{c} / c=0.6$ haben also die Störungsamplituden so große Werte erreicht, dass nichtlineare Effekte aus der Störströmung zu einer Deformation der spannweitig und zeitlich gemittelten Grenzschichtströmung führen.

\subsubsection{Sekundärinstabilität und finaler Umschlag}

Mit der signifikanten Deformation des spannweitig und zeitlich gemittelten Grenzschichtprofils im Vergleich zur Grundströmung und dem Zerfallen einzelner Längswirbel an den am weitesten stromab untersuchten Plattentiefenpositionen wurden deutliche Anzeichen des finalen Umschlags beobachtet. Weitere Indizien sind in den instationären Geschwindigkeitsfluktuationen zu erkennen. Der querströmungsdominierte Umschlag erfolgt stets kurz stromab von Sekundärinstabilitäten [55, 97, 113]. Wie in den Abschnitten 6.2.2 und 6.3.2 beschrieben wurde, sind vor allem hochfrequente Fluktuationen mit Frequenzen $f>1000 \mathrm{~Hz}$ als Hinweis auf den einsetzenden finalen Umschlag zu verstehen, da in diesem Frequenzbereich kaum laufende QSI erwartet werden.

Wie in Abschnitt 2.3 beschrieben, sind in der Literatur Sekundärinstabilitäten drei unterscheidbarer Typen bekannt. Sekundärinstabilitäten vom Typ I treten an Orten großer spannweitiger Scherung auf [71], während Sekun- 


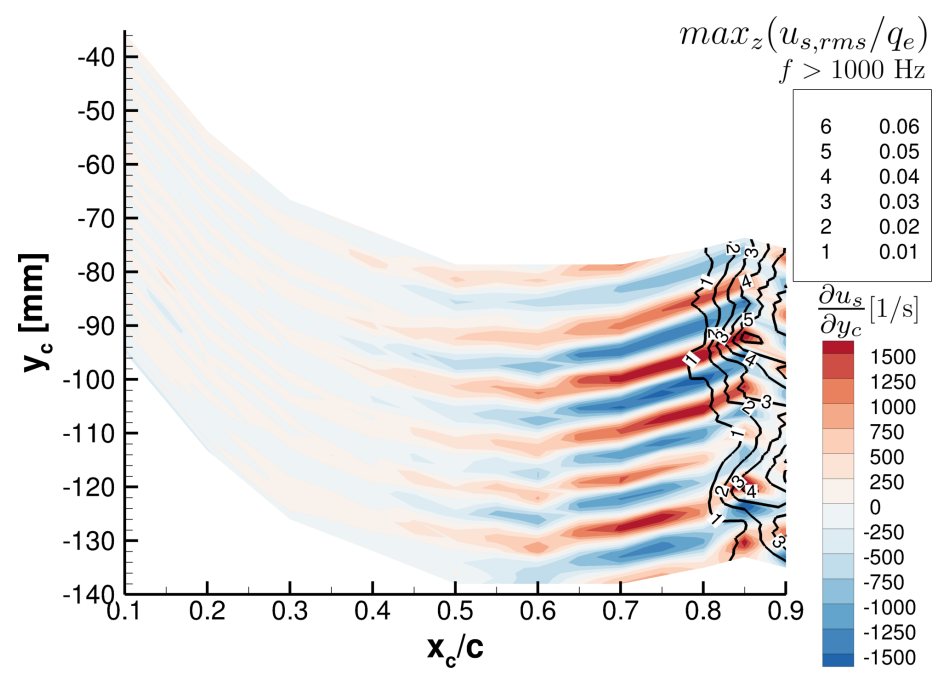

Abbildung 6.3.6: Spannweitige Geschwindigkeitsgradienten der zeitlich gemittelten Komponente $u_{s}$ als Isokonturen mit überlagerten Isolinien des wandnormal maximalen Effektivwertes der instationären Geschwindigkeitsfluktuationen mit $f>1000 \mathrm{~Hz}$

därinstabilitäten vom Typ II und Typ III an Orten großer wandnormaler Scherung auftreten $[54,71]$. Zunächst soll ein Überblick über die Positionen entlang $x_{c}$ und $y_{c}$, an denen hochfrequente Fluktuationen beobachtet wurden, vermittelt werden. Diese Positionen sollen dabei in Relation zur stationären Störströmung gesetzt werden. Zur Visualisierung der stationären Störströmung eignet sich besonders der spannweitige Gradient der zeitlich gemittelten $u_{s}$-Komponente. Um der wachsenden Grenzschichtdicke Rechnung zu tragen, wurde dieser Gradient für die Darstellung in Abb. 6.3.6 je $x_{c}$-Position an dem Wandabstand extrahiert, an dem der spannweitige Mittelwert der $u_{s}$ Komponente dem Wert mean $_{y_{c}}\left(u_{s} / q_{e}\right)=0.7$ am nächsten ist. In dieser Ansicht lassen sich die einzelnen Längswirbel gut in ihrer Entwicklung verfolgen, sobald ihre stationäre Deformation der Grenzschichtprofile ein signifikantes Ausmaß erreicht. Die wandnormal maximalen Effektivwerte instationärer $u_{s^{-}}$ Fluktuationen mit $f>1000 \mathrm{~Hz}$ sind als Isolinien eingezeichnet.

Dieser Effektivwert ist zuerst bei $x_{c} / c=0.8$ signifikant angehoben. Dabei handelt es sich um eine Profiltiefenposition kurz stromab der Position, an der die dominante stationäre QSI-Mode ihre maximale Amplitude erreicht, siehe Abbildung 6.3.2(b). Um der Frage nachzugehen, ob die beobachteten 
Fluktuationen mit einer Sekundärinstabilität assoziiert werden können und um welchen Typ es sich handelt, ist in Abbildung 6.3.7(a) der spannweitige $u_{s}$-Gradient an dieser Position und in Abbildung 6.3.7(b) der wandnormale $u_{s}$-Gradient in Isokonturen dargestellt. Dabei wurde der spannweitige Bereich ausgewählt, in dem die hochfrequenten Fluktuationen mit den größten Amplituden beobachtet wurden. Mit eingezeichnet sind jeweils Isolinien des Effektivwertes von instationären $u_{s}$-Fluktuationen mit $f>1000 \mathrm{~Hz}$. In Abbildung 6.3.7(c) ist die zeitlich gemittelte Komponente $u_{s}$ in Isokonturen dargestellt, wobei zu jedem Wandabstand jeweils ihr spannweitiger Mittelwert abgezogen wurde. Die dargestellten $y_{c}$ - $z$-Schnitte stellen einen Querschnitt durch die Grenzschichtströmung mit Blick in Profiltiefenrichtung dar. Die spannweitig periodischen stationären Längswirbel rotieren in dieser Ansicht gleichsinnig im Uhrzeigersinn, ihr Drehsinn ist in Abbildung 6.3.7(c) schematisch dargestellt. Die Darstellung der Differenz der lokalen $u_{s}$-Komponente von ihrem spannweitigen Mittelwert $\bar{u}_{s}$ offenbart, wie die Längswirbel an ihrer aufsteigenden Flanke impulsarmes Fluid nach oben und an ihrer absteigenden Flanke impulsreiches Fluid nach unten transportiert haben. An der spannweitigen Grenze der absteigenden Flanke eines Längswirbels zur aufsteigenden Flanke des Nachbarwirbels entstehen große spannweitige Geschwindigkeitsgradienten.

In Abbildung 6.3.7(a) ist zu erkennen, dass an Positionen, an denen erhöhte Werte des Effektivwertes hochfrequenter instationärer Fluktuationen beobachtet wurden, auch der spannweitige Gradient $\partial u_{s} / \partial y_{c}$ hohe Werte aufweist. Eine ähnliche örtliche Korrelation mit hohen Werten des wandnormalen Gradienten ist in Abbildung 6.3.7(b) nicht zu erkennen. Die beobachtete örtliche Korrelation mit hohen Beträgen des spannweitigen $u_{s}$-Gradienten ist ein Hinweis darauf, dass es sich bei den hochfrequenten instationären Geschwindigkeitsfluktuationen zumindest teilweise um die Konsequenz einer Sekundärinstabilität vom Typ I handelt. Dieser Typ ist bei querströmungsdominierter Transition fast immer die dominante Sekundärinstabilität und aufgrund ihres starken Wachstums findet der lokale Umschlag zur Turbulenz meist innerhalb von $0.05 c$ stromab ihres ersten Auftretens statt [97].

Vergleicht man die Spektren der instationären Geschwindigkeitsfluktuationen an unterschiedlichen Messpositionen, fallen signifikante Unterschiede besonders bei Frequenzen $f>1000 \mathrm{~Hz}$ auf, wie im Folgenden gezeigt werden soll. Aus dem Bereich angehobener Effektivwerte hochfrequenter Fluktuationen an der aufsteigenden Flanke des Längswirbels zwischen $y_{c}=-94 \mathrm{~mm}$ und $y_{c}=-99 \mathrm{~mm}$ wurden zwei Positionen ausgewählt, für welche die Spektren 
(a)

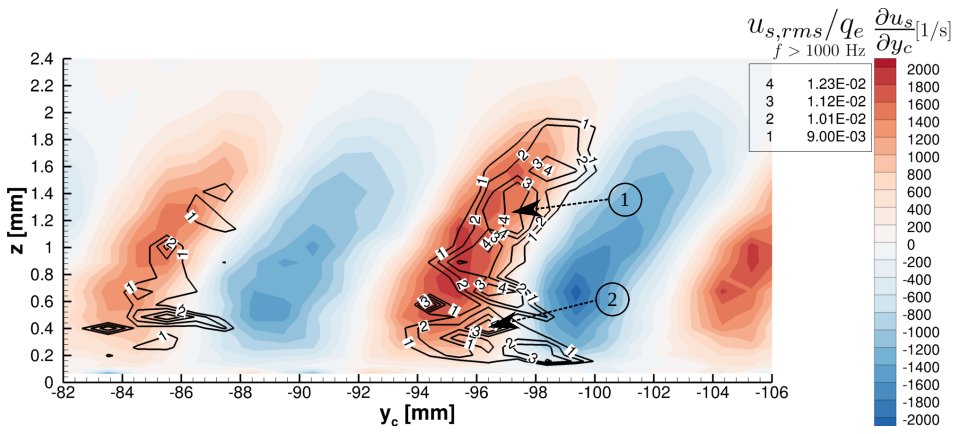

(b)

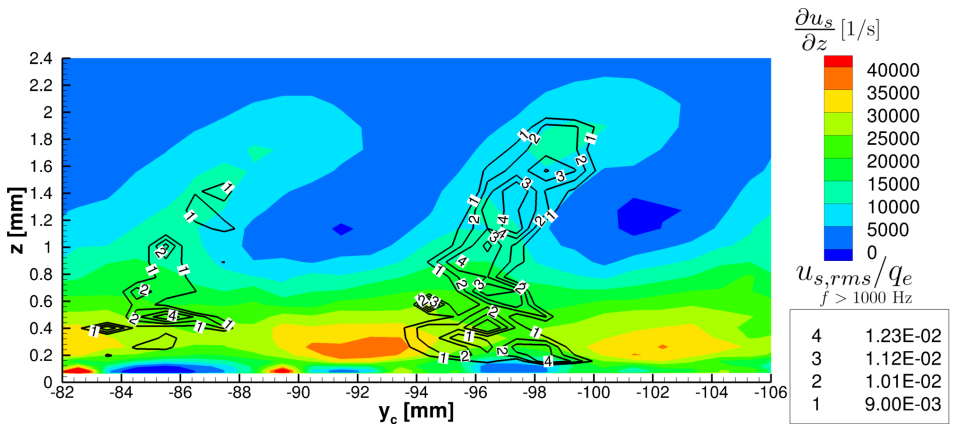

(c)

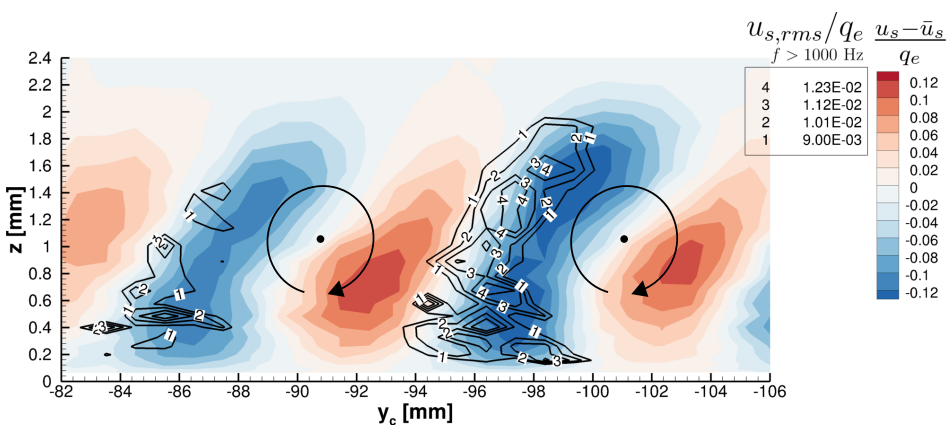

Abbildung 6.3.7: (a) Räumliche Korrelation des Effektivwertes hochfrequenter instationärer $u_{s}$-Fluktuationen mit den Werten des spannweitigen $u_{s}$-Gradienten und (b) mit den Werten des wandnormalen $u_{s}$-Gradienten (c) Visualisierung des Impulstransports durch die Längswirbel, jeweils bei $x_{c} / c=0.8$ 


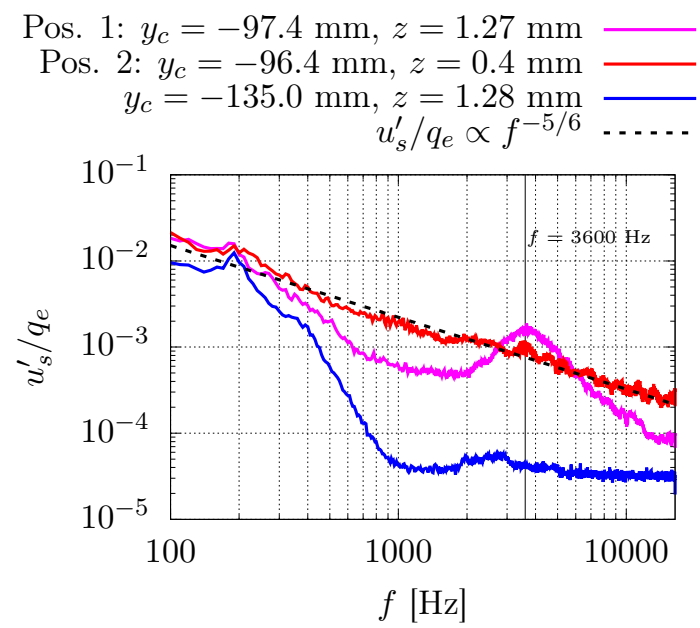

Abbildung 6.3.8: Spektren instationärer Geschwindigkeitsfluktuationen bei $x_{c} / c=0.8$ und unterschiedlichen spannweitigen sowie wandnormalen Positionen im Vergleich

instationärer $u_{s}$-Fluktuationen in Abbildung 6.3.8 abgebildet sind. An Position 1 ( $y_{c}=-97 \mathrm{~mm}$ und $z=1.27 \mathrm{~mm}$ ) ist ein ausgeprägtes lokales Maximum um $f=3600 \mathrm{~Hz}$ zu erkennen. Bereits Lohse et al. [68] identifizierten eine Sekundärinstabilität vom Typ I im neu ausgelegten Prinzipexperiment der schiebenden ebenen Platte, allerdings in einem Fall, in dem eine stationäre QSI mit $\lambda=9 \mathrm{~mm}$ mithilfe von DRE künstlich angeregt war. Auch sie beobachteten ein ähnliches lokales Maximum in Spektren instationärer Fluktuationen an der aufsteigenden Flanke des Längswirbels und identifizierten $f=3500 \mathrm{~Hz}$ als mittlere Frequenz dieses Maximums. Zum Vergleich ist zusätzlich ein Spektrum einer Position am weit entfernten spannweitigen Rand des Messbereichs in einem etwas weniger fortgeschrittenen Bereich des Umschlags dargestellt. Diese Position 3 befindet sich bei $y_{c}=-135 \mathrm{~mm}$ in nahezu gleichem Wandabstand wie Position 1 (im Anhang in Abbildung A.2.1 markiert, vergleiche auch Abb. 6.3.6). In diesem Vergleichsspektrum sind die Schwankungsamplituden für $f>1000 \mathrm{~Hz}$ kaum über das Grundrauschen angehoben.

An Position $2\left(y_{c}=-96.4 \mathrm{~mm}\right.$ und $\left.z=0.4 \mathrm{~mm}\right)$, eine Position mit einem besonders stark angehobenen Effektivwert von Fluktuationen mit $f>1000 \mathrm{~Hz}$, ist solch ein lokales Maximum nicht im Spektrum vorhanden. Stattdessen wird in der gewählten doppelt-logarithmischen Darstellung mit zunehmender 
Frequenz eine nahezu lineare Abnahme von $u_{s}^{\prime}$ beobachtet. In einer turbulenten Grenzschicht zerfallen großskalige turbulente Strukturen zu kleinskaligen Strukturen, deren Energie schließlich zu Wärme dissipiert. In einem gewissen Wellenzahl-Bereich des turbulenten räumlichen Energiespektrums, welcher Inertialbereich genannt wird, ist die kinetische Energie $E_{t}$ abhängig von der räumlichen Wellenzahl $k$ gemäß $E_{t} \propto k^{-\frac{5}{3}}$ verteilt [56]. Hitzdrahtmessungen an einer Position im turbulenten Bereich der Grenzschicht enthalten nicht direkt eine Information über das räumliche Spektrum der turbulenten Strukturen. Wilczek und Narita [114] beschreiben aber basierend auf Arbeiten von Kraichnan [57] ein Modell, in dem sie einen Zusammenhang zwischen dem an einer Messposition mit einer Sonde aufgenommenen zeitlichen turbulenten Spektrum und dem räumlichen Spektrum, welches durch die mittlere Strömung an der Sonde vorbei transportiert wird, herstellen. Ein Ergebnis ist, dass die Energie im zeitlichen Spektrum gemäß $E_{t} \propto f^{-\frac{5}{3}}$ verteilt ist, dass also im zeitlichen Spektrum derselbe Exponent bzgl. der Frequenzabhängigkeit erwartet werden kann wie im räumlichen Spektrum bzgl. der Wellenzahlabhängigkeit.

Da die kinetische Energieamplitude von Fluktuationen quadratisch von ihrer Geschwindigkeitsamplitude $u_{s}^{\prime}$ abhängt, ist in der Abbildung 6.3.8 eine theoretische Amplitudenverteilung gemäß $u_{s}^{\prime} / q_{e} \propto f^{-\frac{5}{6}}$ eingezeichnet. Die Übereinstimmung des Spektrums bei $y_{c}=-96.4 \mathrm{~mm}$ und $z=0.4 \mathrm{~mm}$ mit dieser Proportionalität weist nun darauf hin, dass das Spektrum instationärer Geschwindigkeitsfluktuationen an dieser Position bereits einen turbulenten Charakter besitzt. Bereits White \& Saric beobachteten stark lokalisiert Spektren instationärer Geschwindigkeitsfluktuationen, die einen ,flachen, turbulenten Charakter" besaßen [113]. Wie im hier diskutierten Fall wiesen Spektren an anderen Positionen im Strömungsfeld an derselben Profiltiefenposition keinen solchen Hinweis auf Turbulenz auf. Dies weist auf einen stark lokalisierten turbulenten Umschlag hin. White \& Saric beobachteten den lokalen Umschlag stets kurz stromab der Position, an der zuerst eine Sekundärinstabilität beobachtet wurde und machen diese verantwortlich für den Umschlag. Im hier betrachteten Fall sind die instationären Fluktuationen mit $f>1000 \mathrm{~Hz}$ an der jeweils am weitesten stromauf gelegenen Position Konsequenz einer Sekundärinstabilität. Kurz stromab sind im Effektivwert der Fluktuationen dieses Frequenzbereichs bereits zu einem signifikantem Anteil turbulente Fluktuationen enthalten. 


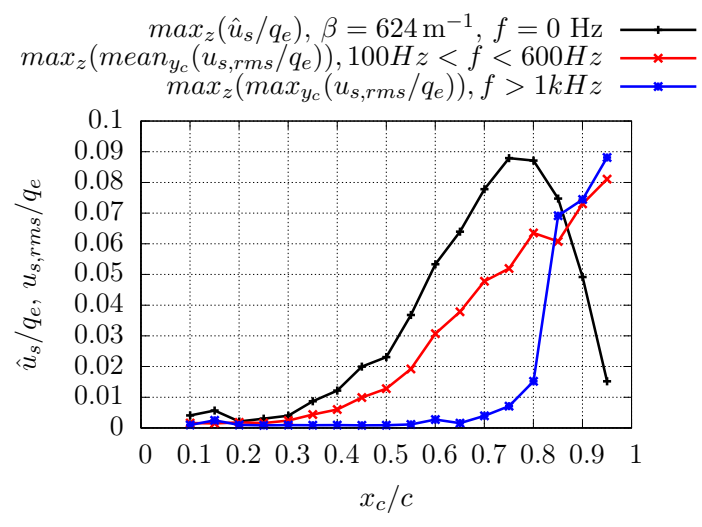

Abbildung 6.3.9: Amplituden der dominanten stationären QSI-Mode, Effektivwerte instationärer $u_{s}$-Fluktuationen repräsentativ für laufende QSI und Effektivwerte repräsentativ für hochfrequente Sekundärinstabilitäten oder turbulente Fluktuationen

\subsubsection{Die verschiedenen Störungen im Überblick}

Nachdem die unterschiedlichen beobachteten Störungen der Grenzschichtströmung einzeln anhand experimenteller Beobachtungen diskutiert und mit theoretischen Erkenntnissen nachvollzogen wurden bzw. im Falle der Sekundärinstabilitäten mit anderen experimentellen Ergebnissen verglichen wurden, soll nun ein Überblick über die unterschiedlichen Störanteile in quantitativer Relation zueinander vermittelt werden. Dazu werden in Abbildung 6.3.9 für jeden der diskutierten Störanteile repräsentative Amplituden und Effektivwerte abhängig von der Profiltiefe $x_{c}$ dargestellt. Wie oben beschrieben, dominiert die stationäre QSI-Mode mit einer spannweitigen Wellenzahl von $\beta=624 \mathrm{~m}^{-1}$ schon weit stromauf das stationäre Störspektrum im Experiment, siehe Abbildung 6.3.2(a). Ihre wandnormal maximal beobachtete Amplitude wurde bereits im Vergleich zur integralen Anfachung der Mode gemäß LST diskutiert. Zum Vergleich ist sie repräsentativ für stationäre Störungen wieder in Abbildung 6.3.9 dargestellt.

Bei der bisherigen Diskussion wurden besonders im Frequenzbereich $100<$ $f<600 \mathrm{~Hz}$ instationäre Fluktuationen mit laufenden QSI assoziiert. Daher ist in Abbildung 6.3.9 das wandnormale Maximum des spannweitig gemittelten Effektivwertes $u_{s, r m s} / q_{e}$ der Bandpass-gefilterten Geschwindigkeitsfluktuationen zwischen $100<f<600 \mathrm{~Hz}$ als bilanzierendes Maß für die Amplitude laufender QSI entlang der Plattentiefe dargestellt. Sobald aber stationäre QSI 
mit signifikanter Amplitude auftreten, können die instationären Störungen nicht mehr rein als primäre QSI im Sinne linearer Stabilitätstheorie interpretiert werden, da nichtlineare Effekte bereits in signifikantem Maß auftreten, siehe auch Abschnitt 2.3.

Des Weiteren wurden besonders im Frequenzbereich $f>1000 \mathrm{~Hz}$ die instationären Fluktuationen als Anzeichen von hochfrequenten Sekundärinstabilitäten und turbulenter Fluktuationen diskutiert. Primäre laufende QSI werden in diesem Frequenzbereich weder erwartet noch beobachtet. Der Effektivwert dieser Fluktuationen ist in Abbildung 6.3.9 als spannweitiger und wandnormaler Maximalwert dargestellt. An der Profiltiefenposition $x_{c} / c=0.8$ ist der Effektivwert signifikant angehoben und es wurden spannweitig und wandnormal lokal sowohl eine Sekundärinstabilität vom Typ I als auch turbulenzähnliche Spektren identifiziert.

Man erkennt, dass der Effektivwert der Geschwindigkeitsschwankungen in dem Frequenzbereich, in dem laufende QSI erwartet und beobachtet werden, entlang fast der ganzen Plattentiefe geringer ist als die Amplitude stationärer QSI mit $\beta=624 \mathrm{~m}^{-1}$. Ab der $x_{c}$-Position des Maximums der dominanten stationären Mode beginnen lokal hochfrequente Schwankungsamplituden zu steigen, was repräsentiert, dass im lokalen Deformationsfeld der stationären QSI erste Sekundärinstabilitäten auftreten. Zwischen $x_{c} / c=0.8$ und $x_{c} / c=0.9$ steigen die Amplituden hochfrequenter Schwankungsanteile aufgrund ihres Wachstums rasch an. Wie in Abschnitt 2.3 beschrieben, folgt der laminar-turbulente Umschlag wegen der hohen Anfachungsraten der Sekundärinstabilitäten immer kurz auf ihr erstes Auftreten. An einzelnen Positionen im Strömungsfeld wurden bereits Spektren instationärer Fluktuationen mit turbulenten Eigenschaften beobachtet. In diesem fortgeschrittenen Stadium der Transition weisen allerdings die einzelnen stationären Längswirbel eine sehr unterschiedliche Stärke auf, sodass der finale Umschlag zunächst stark lokalisiert auftritt. Mit dem Fortschritt des Umschlags auch an anderen Positionen zerfallen die stationären Längswirbel und die spannweitige Periodizität der zeitlich gemittelten $u_{s}$-Komponente mit $\beta=624 \mathrm{~m}^{-1}$ nimmt deutlich ab.

Auf Grundlage der vorgestellten Beobachtungen lässt sich schlussfolgern, dass es sich bei dem betrachteten Fall trotz des in Abschnitt 5.2.1 beschriebenen vergleichsweise hohen Turbulenzgrads des $1 \mathrm{MG}$ noch um einen durch stationäre QSI dominierten Transitionsprozess handelt. Die dominante stationäre QSI mit $\beta=624 \mathrm{~m}^{-1}$ ist über eine Sekundärinstabilität vom Typ I für den laminar-turbulenten Umschlag hauptverantwortlich. 


\section{Kapitel 7}

\section{Beeinflussung durch spannweitig periodische Wandheizung}

Eines der Anregungskonzepte, das im Kontext der UFD-Methode zur Transitionsverzögerung im neu ausgelegten Prinzipexperiment untersucht wird, ist die Anregung durch spannweitig periodische Wandheizung. Eine Hypothese zum Anregungsmechanismus wurde in Abschnitt 2.5.2 und die technische Realisierung in Abschnitt 4.5.1 beschrieben. Der Heizaktuator ist in einen eigenen Naseneinsatz integriert, welcher in die Aufnahmemöglichkeit der Modellplatte eingesetzt wird. An diesem Naseneinsatz wurde eine besondere Oberflächenbeschaffenheit festgestellt, welche zunächst in Abschnitt 7.1 charakterisiert wird. Dies ist besonders daher relevant, da diese Oberfläche bereits ohne künstliche Heizanregung zu einer gegenüber dem Referenzfall veränderten Störströmung führt, welche in Abschnitt 7.2 beschrieben wird.

Die spannweitig periodische Wandheizung geht mit einer Anhebung der spannweitig gemittelten Wandtemperatur einher und erzeugt daher in der Grenzschicht neben der spannweitig periodischen auch eine spannweitig gemittelte Temperaturanhebung. Beides kann die temperatursensitive Hitzdrahtanemometrie beeinflussen und zudem auch die Stabilitätseigenschaften der Grenzschicht verändern. Daher werden diese unmittelbaren Einflüsse in 
Abschnitt 7.3 diskutiert. Anhand einer systematischen Variation der Heizleistung wird dann in Abschnitt 7.4 die künstliche Anregung stationärer QSI mit spannweitig periodischer Wandheizung nachgewiesen und quantifiziert. Des Weiteren wird gezeigt, dass die künstliche stationäre Störungsanregung in ausreichendem Maß isoliert von weiterer Störungsanregung gelingt. Für ausgewählte Anregungsparameter wird dann in Abschnitt 7.5 das Transitionsszenario stromab der Anregung mitsamt der relevanten Störungsströmung charakterisiert. Dabei wird ein Erklärungsansatz dafür präsentiert, warum die künstliche Anregung wider Erwarten zu keiner Transitionsverzögerung führt.

\subsection{Oberflächenbeschaffenheit des Heizaktuators}

Die in Abschnitt 4.5.1 beschriebene konstruktive Realisierung der lokalen Wandheizung machte es nötig, die von den Kupferstrukturen verursachte Rauigkeit durch ein Füllmaterial aus Epoxidharz auszugleichen. Dieses Epoxidharz musste bei Temperaturen ausgehärtet werden, die am oberen Rand der Temperaturbeständigkeit des Naseneinsatz-Hauptmaterials lagen (413 K). Im Anschluss wurde die Oberfläche per Hand geschliffen, um ein möglichst geringes Anregungsniveau durch Rauigkeiten zu erreichen. Da die spektrale Zusammensetzung dieser Rauigkeiten für die Anregung stationärer Querströmungsinstabilitäten im Fall ohne künstliche Heizaktuierung entscheidend ist, soll dieses Spektrum zunächst näher untersucht werden. Dazu wurde das Koordinatenmessgerät „Hexagon Global Performance“ verwendet, welches in der Qualitätssicherung der Feinmechanikwerkstatt des DLR üblicherweise zur Überprüfung der Fertigungsgenauigkeit und Konturtreue von Windkanalmodellen eingesetzt wird. Ein kugelförmiger Tastkopf mit einem Durchmesser von $1 \mathrm{~mm}$ wird in mechanischem Kontakt mit der zu vermessenden Oberfläche entlang einer bestimmten Strecke geführt, wobei ein Signal über die Auslenkung des Tastkopfs und damit über die Oberflächenkontur gewonnen wird. Solche Tastmessungen sind rasterförmig möglich, wodurch eine hochauflösende Vermessung der Oberflächenkontur des Heizaktuator-Naseneinsatzes inklusive seiner Epoxidharzbeschichtung möglich ist. Dabei wurde die Oberfläche, welche die Heizreihen bedeckt, über einem Plattentiefenbereich von $\Delta x_{c}=50 \mathrm{~mm}$ mit 101 Messpunkten und einem spannweitigen Bereich von $\Delta y_{c}=305 \mathrm{~mm}$ mit 607 Messpunkten vermessen (jeweils äquidistant verteilt). Die aufgenommenen Daten ermöglichen es, das zweidimensionale Spektrum 
(a)

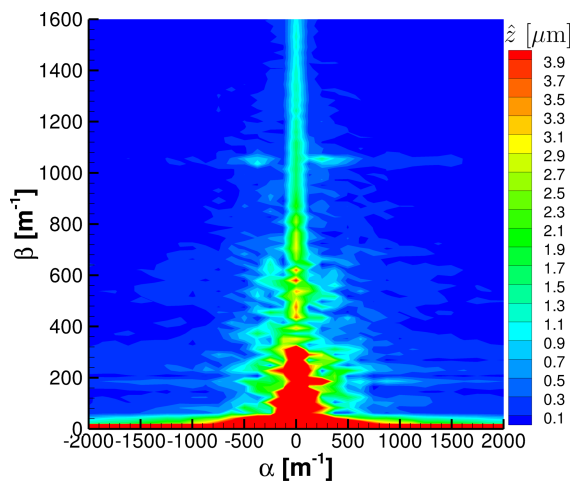

(b)

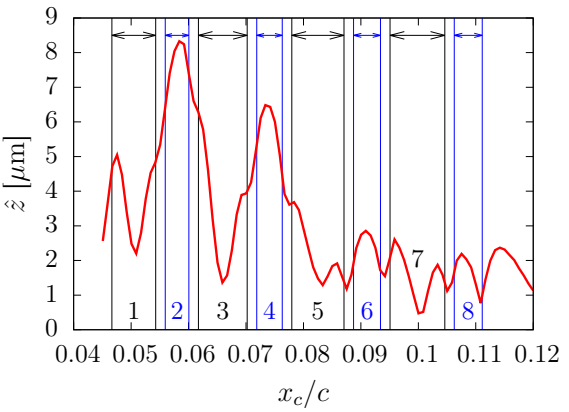

Abbildung 7.1.1: (a) Amplitude $\hat{z}$ der in der Modelloberfläche des Heizaktuators enthaltenen Periodizität abhängig von den spannweitigen und profiltiefenorientierten Wellenzahlen $\beta$ und $\alpha$ (b) Amplitude $\hat{z}$ der Periodizität mit der Aktuierungswellenlänge $\lambda=6 \mathrm{~mm}\left(\beta=1047 \mathrm{~m}^{-1}\right)$ abhängig von der Profiltiefenkoordinate $x_{c} / c$

der Oberflächenkontur zu berechnen und damit eine Information über das Anregungsspektrum für stationäre QSI zu erhalten. Dabei bedingen die verwendeten Schrittweiten und -anzahlen in Spannweiten- und Profiltiefenrichtung eine spektrale Auflösung von $\Delta \beta=20.6 \mathrm{~m}^{-1}$ und $\Delta \alpha=124.4 \mathrm{~m}^{-1}$, wie in Abschnitt 6.3.1 anhand des spannweitigen Spektrums von Geschwindigkeitsvariationen diskutiert. Das resultierende zweidimensionale Spektrum der Rauigkeiten $\hat{z}(\alpha, \beta)$ ist in Abbildung 7.1.1(a) als Isokonturendiagramm dargestellt. Dabei ist ein deutlicher Anteil bei $\beta=1047 \mathrm{~m}^{-1} \mathrm{zu}$ erkennen, der über unterschiedliche $\alpha$-Werte verteilt auftritt. Diese spannweitige Wellenzahl entspricht der spannweitigen Wellenlänge der ausgewählten Kontrollmode $\lambda=6 \mathrm{~mm}$ und damit den spannweitigen Abständen der Heizelemente. Dies verdeutlicht, dass es nicht gelungen ist, die Rauigkeit der Kupferelemente durch die Epoxid-Deckschicht und das anschließende manuelle Schleifen komplett auszugleichen.

Des Weiteren sind signifikante Anteile bei kleineren spannweitigen Wellenzahlen $\beta<600 \mathrm{~m}^{-1}$, also größeren spannweitigen Wellenlängen, erkennbar. Ein Erklärungsansatz hierfür ist, dass beim Aushärten des Epoxidharzes die unterschiedlichen beteiligten Materialien in den Schichten des Heizaktuators durch ihre unterschiedlichen Wärmeausdehnungskoeffizienten eine irreversible Welligkeit der Oberfläche induziert haben. Zu diesen Schichten gehören das 
Hauptmaterial des Einsatzes, das doppelseitige Klebeband, mit dem das flexible Substrat in der Tasche fixiert wurde, das flexible Polyimid-Substrat selbst und die daraufliegenden Kupferstrukturen. Diese Welligkeit konnte durch das nachträgliche Schleifen der Modelloberfläche nicht komplett ausgeglichen werden.

Berechnet man für die 101 spannweitigen Linienmessungen der Oberflächenkontur das spannweitige Spektrum $\hat{z}(\beta)$, lässt sich die Amplitude $\hat{z}$ der spannweitigen Periodizität mit der Wellenlänge $\lambda=6 \mathrm{~mm}$ abhängig von der Profiltiefenkoordinate darstellen und mit der $x_{c}$-Position der Heizreihen vergleichen, siehe Abb. 7.1.1(b). Die Ausdehnung der jeweiligen Heizelemente ist dabei durch Pfeile und die jeweilige Heizreihennummer markiert. Dabei ist erkennbar, dass die Rauigkeit mit der Aktuierungswellenlänge bei Reihen mit langen Heizelementen immer besonders groß am vorderen und hinteren Ende der Heizreihe gemessen wurde. Bei Heizreihen mit kurzen Elementen liegt die maximale Rauigkeit immer nahezu mittig im Profiltiefenbereich der jeweiligen Reihe. Dies ist vermutlich ein Ergebnis der leicht unterschiedlichen Form der kurzen und langen Heizelemente und der Zwischenräume, siehe Abbildung 4.5.3. Sie könnte eine verschiedene Benetzung durch die vor dem Aushärten flüssige Epoxidbeschichtung bewirken.

Der über den vermessenen Profiltiefenbereich gemittelte Mittenrauwert beträgt $\bar{R}_{a}=2.5(9) \mu \mathrm{m}$. Die Oberfläche des Naseneinsatzes besitzt also einen höheren mittleren Mittenrauwert $R_{a}$ als der Naseneinsatz mit Druckbohrungen mit $\bar{R}_{a}=0.5(1) \mu \mathrm{m}$, siehe auch Abschnitt 4.4. Für die folgende Interpretation des Störungsspektrums in der Grenzschichtströmung sind aber vor allem die auffälligen langwelligen Anteile in der spannweitigen Periodizität von Bedeutung.

\subsection{Die Störströmung stromab des ausgeschalteten Heizaktuators}

Der Heizaktuatoreinsatz besitzt dieselbe nominelle Kontur wie der Naseneinsatz, für den die Grenzschichtströmung in Abschnitt 6.3 als Referenzfall beschrieben wurde. Im Plattentiefenbereich $0 \leq x_{c} / c \leq 0.25$ ist die Messung der Wanddruckverteilung nicht möglich, da in den Aktuatoreinsätzen keine Druckbohrungen vorgesehen sind. Hitzdrahtmessungen am Grenzschichtrand zeigen aber, dass die Verläufe der Grenzschichtrandgeschwindigkeiten $u_{c, e}\left(x_{c}\right)$ und $v_{c, e}\left(x_{c}\right)$ keine signifikanten Unterschiede abhängig vom verwendeten 
Naseneinsatz aufweisen. Daher wird im Folgenden angenommen, dass die Grenzschichtgrundströmung im ungeheizten Fall der in Abschnitt 6.3 beschriebenen Grenzschichtströmung entspricht, zumindest stromauf der nichtlinearen Deformation der spannweitig gemittelten Grenzschichtprofile aufgrund der Störströmung.

Der Einfluss der im spannweitigen Mittel angehobenen Wandtemperatur wird in Abschnitt 7.3.1 besonders mit Blick auf die Messungen zur systematischen Heizleistungsvariation diskutiert. Durch die spezielle Oberfläche des Heizaktuators und dem damit verbundenen stationären Anregungsspektrum ist das Transitionsszenario bereits im ausgeschalteten Zustand des Aktuators gegenüber dem in Abschnitt 6.3 beschriebenen Szenario signifikant verändert. Um später den Einfluss der Aktuierung verstehen zu können, ist eine genaue Kenntnis dieses veränderten Szenarios bzgl. der Störströmung nötig. Ein Vergleich der aktuierten Störströmung stromab des Heizaktuators mit der Störströmung des Referenzfalls ganz ohne Aktuator würde zu Fehlinterpretationen führen. Daher wurden in der Grenzschicht an der Modellplatte auf und stromab des Naseneinsatzes rasterförmige Hitzdrahtmessungen mit ausgeschaltetem Aktuator durchgeführt. Dazu wurde die Hitzdrahtsonde an 14 in $x_{c}$-Richtung verteilten Positionen an je 61 spannweitig verteilten und 30 wandnormal verteilten Positionen positioniert. Für weitere Details zum typischen Messablauf dreidimensionaler Rastermessungen siehe Abschnitt 5.1.4.

\subsection{1 Überblick über die Störungsentwicklung}

Für einen Überblick über die Grenzschichtströmung stromab des ausgeschalteten Heizaktuators ist in Abbildung 7.2.1(a) die zeitlich gemittelte $u_{s}$-Geschwindigkeitskomponente aus den rasterförmigen Hitzdrahtmessungen als Isokonturen dargestellt. Man erkennt deutlich die Deformation der Grenzschicht durch stationäre QSI. Im Vergleich mit dem Referenzfall (siehe Abbildung 6.3.1) fällt bereits auf, dass die lokale Deformation der Grenzschichtprofile spannweitig besonders langwellige spektrale Inhalte besitzt. Dazu ist in Abbildung 7.2.1(b) das Ergebnis der Fouriertransformation des spannweitigen Verlaufs der $u_{s}$-Komponente als jeweils wandnormal maximale Amplitude $\hat{u}_{s} / q_{e}$ abhängig von der spannweitigen Wellenzahl $\beta$ und der Plattentiefe $x_{c} / c$ dargestellt. Dabei ist wieder die spektrale Auflösung $\beta=104.05 \mathrm{~m}^{-1} \mathrm{zu}$ beachten, siehe Abschnitt 6.3.1. Es ist zu erkennen, dass die Wellenzahl der dominanten stationären QSI nicht wie im Referenzfall 
(a)

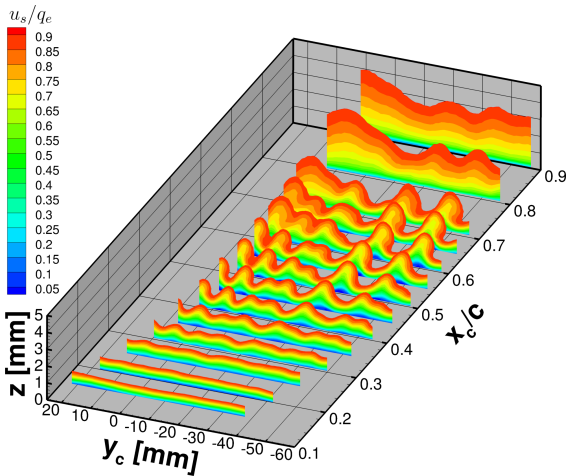

(b)

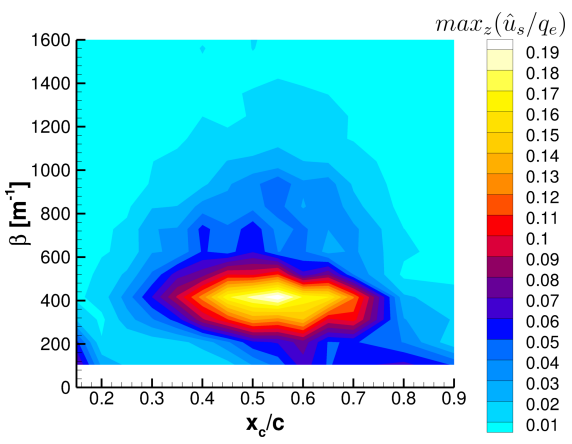

Abbildung 7.2.1: (a) Durch stationäre QSI deformiertes Grenzschichtströmungsfeld im Fall des ausgeschalteten Heizaktuators (b) Wandnormal maximal beobachtete Amplituden spannweitiger Periodizität der Grenzschichtdeformation, abhängig von der spannweitigen Wellenzahl $\beta$ und der Plattentiefenkoordinate $x_{c} / c$

$\beta=624 \mathrm{~m}^{-1}$ beträgt, sondern $\beta=416 \mathrm{~m}^{-1}$ (vergleiche Abb. 6.3.2(a)), auch wenn im Wellenzahlbereich der im Referenzfall dominanten stationären QSI ebenfalls signifikante Amplituden beobachtet werden. Im vorangegangenen Abschnitt 7.1 wurde beschrieben, dass das Rauigkeitsspektrum des Heizaktuator-Naseneinsatzes auffällig hohe Anteile bei niedrigen spannweitigen Wellenzahlen $\beta<600 \mathrm{~m}^{-1}$ aufweist, siehe insb. Abb. 7.1.1(a). Auch wenn dort kein ausgeprägtes lokales Maximum bei $\beta=416 \mathrm{~m}^{-1}$ beobachtet wurde, werden diese spektralen Anteile für das veränderte stationäre Störspektrum stromab des ausgeschalteten Heizaktuators verantwortlich gemacht.

Zur Übersicht über die beteiligten Störungsanteile sind in Abbildung 7.2.2(a) die wandnormal maximalen Amplituden der spannweitigen stationären $u_{s^{-}}$ Deformation mit einer spannweitigen Wellenzahl von $\beta=416 \mathrm{~m}^{-1}$ und $\beta=624 \mathrm{~m}^{-1}$ dargestellt. Mit eingezeichnet sind die spannweitig gemittelten und wandnormal maximalen Effektivwerte zeitlicher Geschwindigkeitsschwankungen zwischen $100 \mathrm{~Hz}$ und $600 \mathrm{~Hz}$, welche in Abschnitt 6.3 .2 besonders mit laufenden QSI assoziiert wurden. Wie schon in Abschnitt 6.3.2 beschrieben, repräsentiert der Effektivwert instationärer $u_{s}$-Fluktuationen mit Frequenzen $f>1000 \mathrm{~Hz}$ entweder hochfrequente sekundäre Instabilitäten oder bereits turbulente Fluktuationen. Dieser Effektivwert ist je $x_{c}$-Position als spannweitiger und wandnormaler Maximalwert dargestellt. In manchen der dargestellten 
(a)

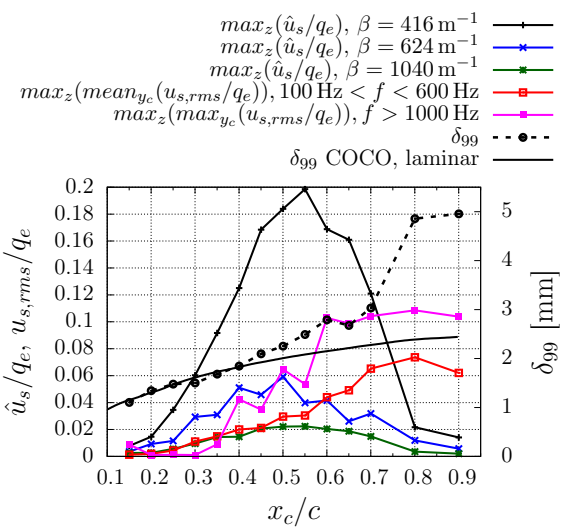

(b)

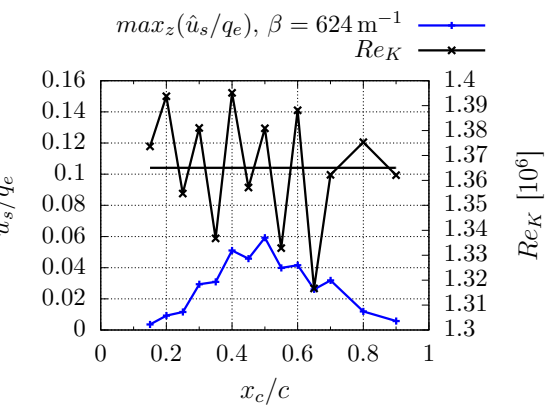

Abbildung 7.2.2: (a) Entwicklung stationärer und instationärer Störungsgrößen stromab des ausgeschalteten Heizaktuators (b) „Örtliche“ Korrelation der gemessenen Störamplitude mit der Windkanal-Reynoldszahl aufgrund der Messreihenfolge

Verläufe sind auffällige Sprünge erkennbar, die später in diesem Abschnitt diskutiert werden.

Die stationäre QSI-Mode mit $\beta=416 \mathrm{~m}^{-1}$ wächst in ihrer Amplitude rasch mit $x_{c}$ an und erreicht ihren maximalen Wert von etwa $\hat{u}_{s} / q_{e}=0.2$ bei $x_{c} / c=0.55$. Stromab nimmt ihre Amplitude wieder stark ab. Diese Mode besitzt entlang der gesamten Profiltiefe deutlich höhere Amplituden als die Mode mit $\beta=624 \mathrm{~m}^{-1}$, welche im Referenzfall die stationäre Störströmung dominierte. Zudem ist der Wert $\hat{u}_{s} / q_{e}=0.2 \mathrm{mehr}$ als doppelt so groß wie der im Referenzfall maximal erreichte Wert $\hat{u}_{s} / q_{e}=0.09$ (vergleiche Abbildung 6.3.9). Die QSI-Mode mit $\beta=624 \mathrm{~m}^{-1}$ erreicht stromab des ausgeschalteten Heizaktuators nur eine maximale Amplitude von $\hat{u}_{s} / q_{e}<0.06$ bei $x_{c} / c=0.5$. Ebenso erkennt man, dass die in Abschnitt 7.1 beschriebene Rauigkeitsanregung bei der spannweitigen Wellenlänge $\lambda=6 \mathrm{~mm}$ zu einer Instabilitätsamplitude von maximal $\hat{u}_{s} / q_{e}=0.02$ bei $\beta=1040 \mathrm{~m}^{-1}$ führt (unter Beachtung der spektralen Auflösung des spannweitigen $\hat{u}_{s}$-Spektrums).

Der spannweitig gemittelte und wandnormal maximale Effektivwert instationärer Geschwindigkeitsschwankungen mit $100 \mathrm{~Hz}<f<600 \mathrm{~Hz}$ zeigt im größten Teil der Profiltiefe ein kontinuierliches Wachstum und erreicht ab $x_{c} / c=0.7$ einen Plateauwert mit $7 \%$ bis $8 \%$ der Grenzschichtrandgeschwindigkeit $q_{e}$. Der spannweitig und wandnormal maximale Effektivwert hochfre- 
quenter Fluktuationen beginnt schon bei $x_{c} / c=0.35$ zu steigen. An dieser Plattentiefenposition sind keine Anzeichen des finalen Umschlags zu erkennen. Die Amplituden der stationären QSI wachsen weiter an und die spannweitig mittlere Grenzschichtdicke folgt weiter dem laminaren Verlauf. Daher wird der angehobene Effektivwert nicht als Anzeichen turbulenter Fluktuationen interpretiert, sondern als Anzeichen von Sekundärinstabilitäten, wie später gezeigt wird. Das Transitionsszenario zeigt in diesem Fall kein so eindeutiges Anzeichen für den finalen Umschlag wie im Referenzfall, in dem der Effektivwert hochfrequenter Fluktuationen entlang des Großteils der Plattentiefe einen geringen Betrag besaß und auf sein rapides Anschnellen im spannweitigen und wandnormalen Maximum kurz stromab der finale Umschlag folgte. Im hier betrachteten Fall lässt sich die spannweitig gemittelte Transitionslage unter anderem am deutlichen Aufdicken der Grenzschicht ab $x_{c} / c=0.65$ erkennen. Bereits ab $x_{c} / c=0.55$ ist eine Abnahme der Amplitude der dominanten stationären QSI zu erkennen, da einzelne stationäre Längswirbel im Rahmen des finalen Umschlags zerfallen, ähnlich zum Referenzfall (siehe Abbildung 6.3.9). Bei $x_{c} / c=0.6$ wird im spannweitigen und wandnormal maximalen Effektivwert hochfrequenter Fluktuationen näherungsweise ein Plateauwert erreicht, welcher bis zur letzten untersuchten Plattentiefenposition nahezu konstant bleibt. Der Betrag dieses Plateauwertes ist höher als der im Referenzfall maximal erreichte, was darauf hinweist, dass an der letzten untersuchten Profiltiefenposition der finale Umschlag weiter fortgeschritten ist als im Referenzfall. Diese Beobachtung wird dadurch unterstützt, dass bei $x_{c} / c=0.8$ und $x_{c} / c=0.9$ eine spannweitig mittlere Grenzschichtdicke $\delta_{99}$ von nahezu $5 \mathrm{~mm}$ ermittelt wird, ein etwa doppelt so großer Wert wie an der letzten untersuchten Profiltiefenposition im Referenzfall (vgl. Abb. 6.3.5).

In den Verläufen der stationären QSI-Mode mit $\beta=624 \mathrm{~m}^{-1}$ und der instationären Fluktuationen mit $f>1000 \mathrm{~Hz}$ sind Sprünge entlang $x_{c}$ zu erkennen. Eine sehr wahrscheinliche Erklärung liefert die Messreihenfolge. Zunächst wurden die rasterförmigen Hitzdrahtmessungen bei den Positionen $x_{c} / c=$ $0.2,0.3,0.4,0.5,0.6,0.7,0.8$ und $x_{c} / c=0.9$ durchgeführt. Im Anschluss wurden die Messungen bei den Positionen $x_{c} / c=0.15,0.25,0.35,0.45,0.55$ und $x_{c} / c=0.65$ durchgeführt, sodass sich ein vergleichsweise großer zeitlicher Abstand von bis zu einer Woche zwischen Messungen an benachbarten $x_{c^{-}}$ Positionen ergibt. Durch den großen zeitlichen Abstand unterscheiden sich die Umweltbedingungen während der Experimente und damit die in Gleichung 5.2.1 definierte Windkanal-Reynoldszahl $R e_{K}$. Diese ist in Abbildung 7.2.2(b) gemeinsam mit der wandnormal maximalen Amplitude der stationären QSI mit $\beta=624 \mathrm{~m}^{-1}$ dargestellt. $R e_{K}$ wurde dabei über alle Messpositionen der 
Ebene an der jeweiligen Plattentiefenposition $x_{c}$ gemittelt. Wie in Abschnitt 5.2.2 beschrieben wurde, ist die Standardabweichung von $R e_{K}$ während der Sommer-Messkampagne, in der die Experimente mit der Heizaktuierung durchgeführt wurden, kleiner als 2\%. Allerdings ist in Abbildung 7.2.2(b) eine örtliche bzw. eigentlich messungsbezogene Korrelation der Störamplitude mit dem jeweiligen gemittelten $R e_{K}$-Wert zu beobachten. Dies legt die Interpretation nahe, dass die geringen Schwankungen in $R e_{K}$ einen nicht zu vernachlässigenden Einfluss auf das jeweilige Störungsniveau haben. Leider kann mit der Drehzahl-Regelung des Gebläses am 1MG nur die Anströmgeschwindigkeit $q_{K, \infty}$ konstant gehalten werden, nicht $R e_{K}$.

\subsubsection{Instationäre Störungen im frühen Stadium}

Im Folgenden wird diskutiert, inwiefern die im Vergleich zum Referenzfall geänderte Modelloberfläche des Heizaktuators das instationäre Störungsspektrum beeinflusst. Im Referenzfall konnte durch den Vergleich der Spektren spannweitig gemittelter und wandnormal maximaler instationärer $u_{s}$-Fluktuationsamplituden mit den theoretischen Verteilungen maximal erwarteter Amplituden $u_{A}^{\prime}\left(x_{c}, f\right)$ besonders der Frequenzbereich $100<f<600 \mathrm{~Hz}$ mit laufenden QSI assoziiert werden, siehe Abschnitt 6.3.2.

Analog dazu ist in Abbildung 7.2.3 das Spektrum der wandnormal maximalen und spannweitig gemittelten instationären Schwankungsamplituden an unterschiedlichen $x_{c}$-Positionen im Vergleich zum Referenzfall dargestellt. Wieder sind auch die Verteilungen theoretisch maximal erwarteter Amplituden laufender QSI an denselben Profiltiefenpositionen dargestellt. Dabei wurde $r_{K}$ so gewählt, dass $u_{A}^{\prime}\left(x_{c}, f\right) / q_{e}$ bei $x_{c} / c=0.25$ in einem möglichst breiten Frequenzbereich mit dem experimentellen Spektrum $\max _{z}\left(\operatorname{mean}_{y_{c}}\left(u_{s}^{\prime} / q_{e}\right)\right)$ übereinstimmt. Bei $x_{c} / c=0.2$ hat das experimentelle Spektrum stromab des ausgeschalteten Heizaktuators wie im Referenzfall noch keine Ähnlichkeit mit $u_{A}^{\prime}\left(x_{c}, f\right)$. Die Amplituden sind im Vergleich zum Referenzfall zwischen 100 und $400 \mathrm{~Hz}$ angehoben. Bei $x_{c} / c=0.25$ werden im gesamten Frequenzbereich $100 \mathrm{~Hz}<f<600 \mathrm{~Hz}$ höhere Amplituden beobachtet als im Referenzfall. Wie im Referenzfall lässt sich anhand des Vergleichs mit $u_{A}^{\prime}\left(x_{c}, f\right)$ die obere Grenzfrequenz integral angefachter laufender QSI nachvollziehen. Allerdings werden gleichzeitig bei Frequenzen $f<300 \mathrm{~Hz}$ höhere Amplituden beobachtet als mit $u_{A}^{\prime}\left(x_{c}, f\right)$ unter den in Abschnitt 6.3.2 geschilderten Annahmen erwartet. Auch bei $x_{c} / c=0.3$ ist die obere Grenzfrequenz laufender QSI nachzuvollziehen. Die besonders auffälligen hohen Amplituden bei Frequenzen 
(a) $x_{c} / c=0.2$

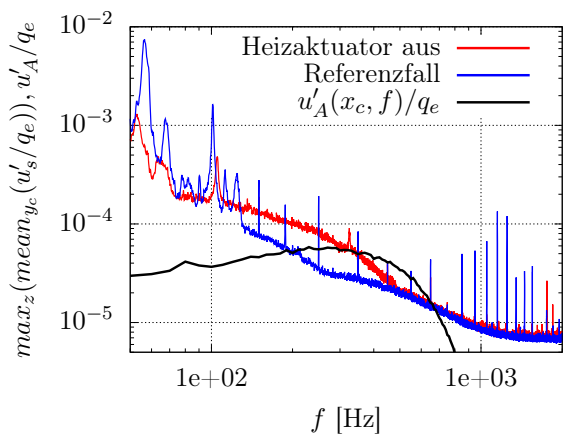

(b) $x_{c} / c=0.25$

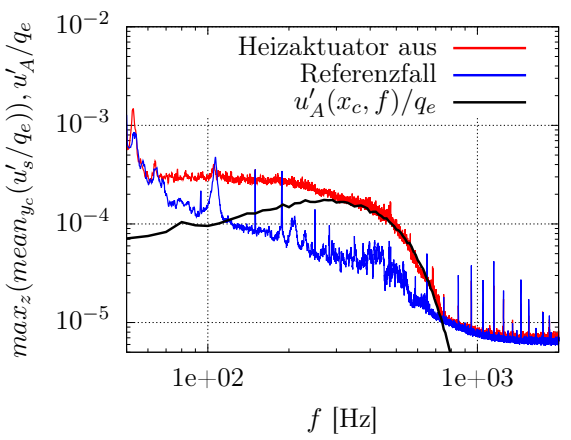

(c) $x_{c} / c=0.3$

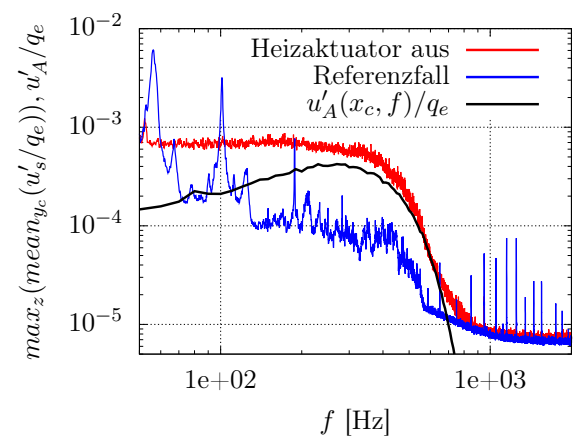

Abbildung 7.2.3: Vergleich des wandnormal maximalen und spannweitig gemittelten Spektrums stromab des ausgeschalteten Heizaktuators mit dem äquivalenten Spektrum im Referenzfall bei der jeweiligen Plattentiefenposition 
$f<300 \mathrm{~Hz}$ sind weiter angewachsen. Wie in Abschnitt 5.2.1 beschrieben wurde, sind in der Anströmung signifikante spektrale Schwankungsanteile bei niedrigen Frequenzen enthalten und ihre Amplitude nimmt mit der Frequenz monoton ab. Die Tatsache, dass $u_{A}^{\prime}\left(x_{c}, f\right)$ zwischen $200 \mathrm{~Hz}$ und $300 \mathrm{~Hz}$ ein Maximum besitzt, ist eine Konsequenz aus der vglw. geringen integralen Anfachung für laufende QSI mit $f<300 \mathrm{~Hz}$ gemäß der LST-Untersuchung (siehe auch Abschnitt 6.2.2).

Die wesentlichen beobachteten Unterschiede zwischen dem Transitionsszenario stromab des ausgeschalteten Heizaktuators und dem im Referenzfall sind die größere Wellenlänge der dominanten stationären QSI und deren höhere Amplituden. Wie bereits oben beschrieben, besitzt der Heizaktuatoreinsatz durch seine veränderte Oberflächenbeschaffenheit ein gegenüber dem Referenzfall verändertes Anregungsspektrum für stationäre QSI, was zu einem veränderten beobachteten Spektrum der angeregten stationären QSI führt. Es ist bekannt, dass bei der Anregung laufender QSI instationäre Fluktuationen aus der Anströmung im Rezeptivitätsprozess mit der Modelloberfläche interagieren [14]. Dabei ist das Rauigkeitsspektrum auch für die angeregten laufenden QSI wichtig. Ein Erklärungsansatz für die beobachteten erhöhten Fluktuationsamplituden im Frequenzbereich laufender primärer QSI ist daher ein durch das veränderte Rauigkeitsspektrum verstärktes Einkoppeln der instationären Fluktuationen als laufende QSI. Dies ließe sich aber nur durch eine Untersuchung des spannweitigen Spektrums der laufenden QSI im linearen Bereich ihrer Störungsentwicklung nachweisen, was mit den zur Verfügung stehenden Messdaten nicht möglich ist.

Ein anderer Erklärungsansatz ist, dass es sich bei den beobachteten erhöhten Amplituden bei Frequenzen unter $600 \mathrm{~Hz}$ um niederfrequente sekundäre Instabilitäten im durch die langwelligen stationären QSI deformierten stationären Strömungsfeld handelt. Die sekundäre Anfachung bzgl. dieses Frequenzbereichs könnte stärker als im Referenzfall und insbesondere stärker als die primäre Anfachung sein und damit die angehobenen Amplituden erklären. Die dominierenden langwelligen stationären QSI erreichen im Fall des ausgeschalteten Heizaktuators im Gegensatz zu den dominanten stationären QSI im Referenzfall bereits bei $x_{c} / c=0.25$ signifikante Amplituden. Des Weiteren ist auch möglich, dass beide Erklärungsansätze teilweise zu den angehobenen Amplituden instationärer Fluktuationen im Frequenzbereich $100 \mathrm{~Hz}<f<600 \mathrm{~Hz}$ beitragen. 
(a) Referenzfall

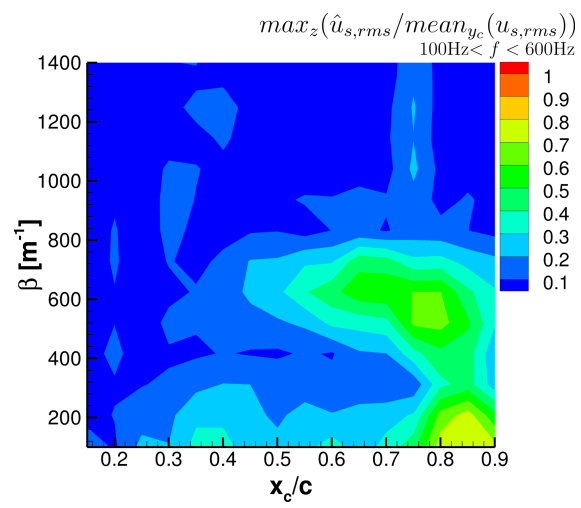

(b) Ausgeschalteter Heizaktuator

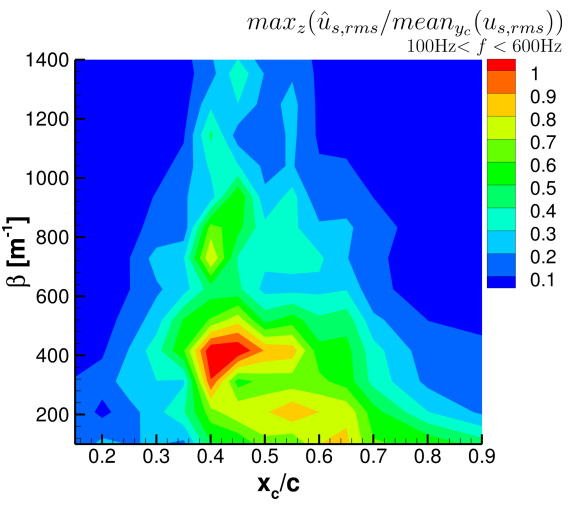

Abbildung 7.2.4: Amplituden der spannweitigen Periodizität der Effektivwerte zeitlicher Geschwindigkeitsfluktuationen zwischen $100 \mathrm{~Hz}<f<600 \mathrm{~Hz}$, für (a) den Referenzfall und (b) für den Fall mit ausgeschaltetem Heizaktuator

Wären die bereits bei $x_{c} / c=0.25$ angehobenen Amplituden bei Frequenzen zwischen $100 \mathrm{~Hz}$ und $600 \mathrm{~Hz}$ eine Konsequenz einer sekundären Instabilität, müssten die Effektivwerte in diesem Frequenzbereich bereits eine ausgeprägte spannweitige Periodizität aufweisen. Ähnlich wie zur Diskussion des stationären Störungsspektrums wurde die spannweitige Verteilung der Effektivwerte gemessener instationärer Geschwindigkeitsfluktuationen daher bzgl. ihrer spannweitigen Periodizität untersucht. Das räumliche Spektrum bzgl. der spannweitigen Wellenzahl $\beta$ des Effektivwerts von Fluktuationen im Frequenzbereich $100 \mathrm{~Hz}<f<600 \mathrm{~Hz}$ wurde für alle untersuchten Wandabstände gebildet. Das wandnormale Maximum der ermittelten Amplituden $\hat{u}_{s, r m s}$ dieser spannweitigen Periodizität in Abhängigkeit der spannweitigen Wellenzahl $\beta$ und der Plattentiefenposition $x_{c} / c$ ist in Abbildung 7.2.4 im Vergleich für den Referenzfall und den Fall stromab des ausgeschalteten Heizaktuators dargestellt. Die Amplitude wurde dabei auf den jeweiligen spannweitigen Mittelwert bezogen. Dabei erkennt man, dass im Fall des ausgeschalteten Heizaktuators im Gegensatz zum Referenzfall der Effektivwert der Fluktuationen in diesem Frequenzbereich weiter stromauf eine ausgeprägte spannweitige Periodizität aufweist. Ab $x_{c} / c=0.3$ ist eine signifikante spannweitige Periodizität dieser Fluktuationen zu erkennen, besonders im langwelligen Wellenzahlbereich der dominanten stationären QSI. Die dominante Mode stationärer QSI mit $\beta=416 \mathrm{~m}^{-1}$ besitzt bei $x_{c} / c=0.3$ eine Amplitude von etwa $6 \%$ der Grenzschichtrandgeschwindigkeit, siehe Abb. 7.2.2(a). Ab 


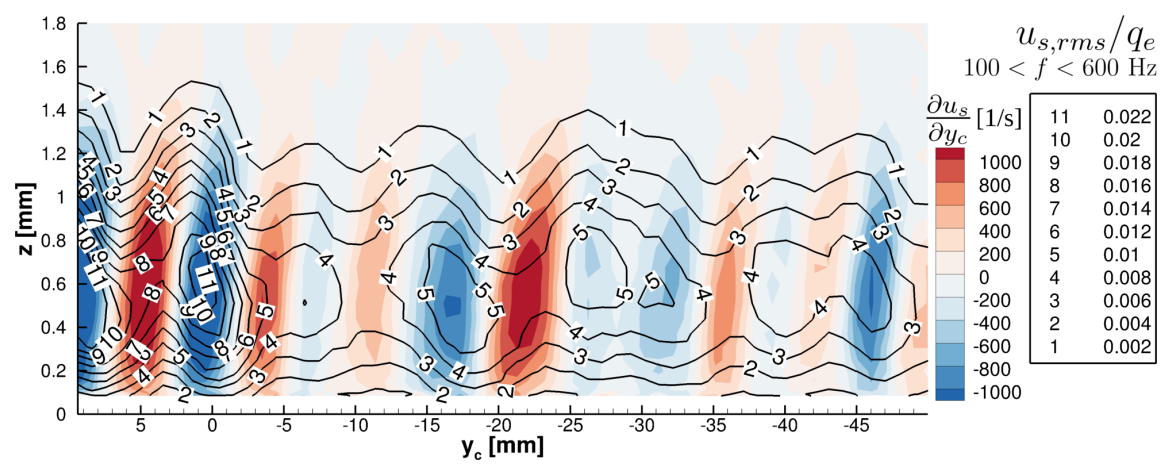

Abbildung 7.2.5: Effektivwerte der Fluktuationen zwischen $100 \mathrm{~Hz}$ und $600 \mathrm{~Hz}$ als Isolinien und Werte des spannweitigen Gradienten der stationären Geschwindigkeitskomponente $u_{s}$ in farblichen Isokonturen bei $x_{c} / c=0.3$

dieser Plattentiefenposition ist also zu erwarten, dass die Effektivwerte der instationären Fluktuationen, welche im Referenzfall mit laufenden QSI assoziert wurden, örtlich mit dem durch stationäre QSI deformierten stationären Grenzschichtströmungsfeld korrelieren.

In Abbildung 7.2.5 ist der beobachtete Effektivwert der Fluktuationen zwischen $100 \mathrm{~Hz}$ und $600 \mathrm{~Hz}$ bei $x_{c} / c=0.3$ als Isolinien in Relation zum lokalen Gradienten $\partial u_{s} / \partial y_{c}$ dargestellt, welcher in farblichen Isokonturen dargestellt ist. Dabei erkennt man, dass diese instationären Fluktuationen nicht wie für laufende QSI erwartet mit spannweitig gleichverteilter Amplitude auftreten. Stattdessen wird der Effektivwert mit spannweitig deutlich variierendem Wert beobachtet. Die gegenüber dem spannweitigen Mittelwert angehobenen Effektivwerte korrelieren örtlich mit lokalen Minima des spannweitigen Gradienten $\partial u_{s} / \partial y_{c}$.

Auf Basis der vorliegenden Messungen lässt sich zusammenfassend nicht klarstellen, ob die angehobenen Fluktuationsamplituden im Frequenzbereich $100 \mathrm{~Hz}<f<600 \mathrm{~Hz}$ und im Profiltiefenbereich $0.2<x_{c} / c<0.3$ auch ein Rezeptivitätseffekt sind. Die starke Deformation der Grenzschicht durch die langwelligen stationären QSI sorgt dafür, dass diese instationären Fluktuationen spannweitig moduliert als niederfrequente Sekundärinstabilität auftreten. Dadurch ist die Anfachung der instationären Störungen möglicherweise verstärkt, was die beobachteten höheren Amplituden als im Referenzfall erklären kann. 


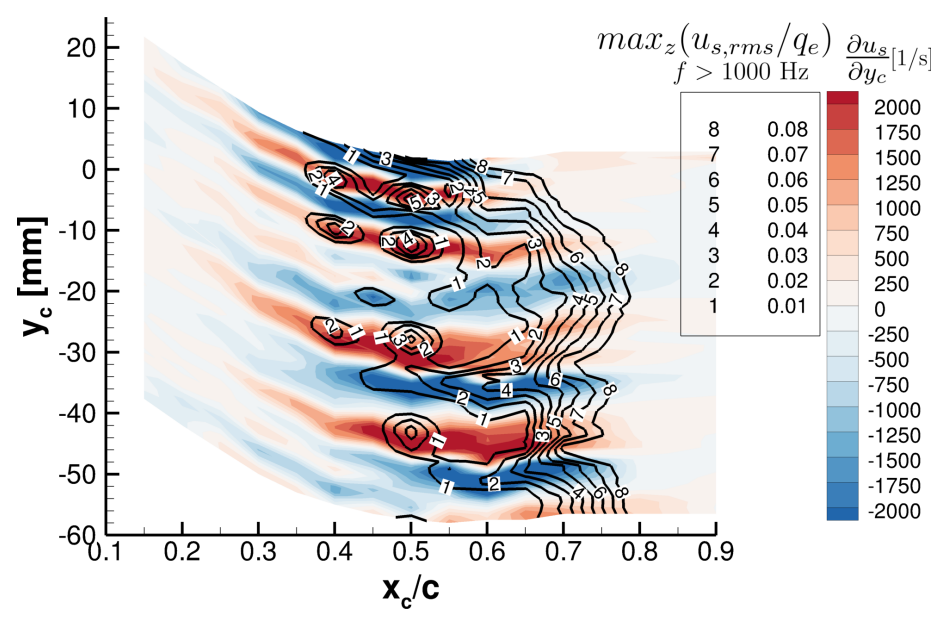

Abbildung 7.2.6: Spannweitige Gradienten $\frac{d u_{s}}{d y_{c}}$ als Visualisierung der stationären QSI und wandnormal maximale Effektivwerte hochfrequenter $u_{s}$-Schwankungen mit $f>1000 \mathrm{~Hz}$ als Isolinien

\subsubsection{Hochfrequente instationäre Störungen und Sekundärinstabilität}

Analog zur Diskussion des Transitionsszenarios im Referenzfall (Abb. 6.3.6), ist in Abb. 7.2.6 der spannweitige Gradient der zeitlich gemittelten $u_{s}$-Komponente in farblichen Isokonturen für den Fall stromab des ausgeschalteten Heizaktuators dargestellt. Dabei wurde der Wert das Gradienten wieder zu jeder Profiltiefenposition an dem Wandabstand extrahiert, an dem der spannweitige Mittelwert der zeitlich gemittelten $u_{s}$-Komponente dem Wert mean $_{y_{c}}\left(u_{s} / q_{e}\right)=0.7$ am nächsten ist. Als Isolinien ist wieder der wandnormal maximale Effektivwert instationärer Geschwindigkeitsfluktuationen mit einer Frequenz von $f>1000 \mathrm{~Hz}$ eingezeichnet. Somit ergibt sich eine Aufsichtsdarstellung auf die transitionelle Grenzschicht. Dabei ist deutlich, dass die Störströmung im Vergleich zum Referenzfall verändert ist. Im Plattentiefenbereich $0.15<x_{c} / c<0.3$ erkennt man noch eine vergleichsweise kurzwellige spannweitige Periodizität im spannweitigen $u_{s}$-Gradienten. Stromab dominiert die bereits diskutierte langwellige spannweitige Periodizität. Dieses gegenüber dem Referenzfall veränderte spannweitige Spektrum stationärer QSI liegt wie oben diskutiert an der in Abschnitt 7.1 beschriebenen Anregung durch Rauigkeit. 
$\mathrm{Ab} x_{c} / c=0.4$ werden erstmals erhöhte Werte des Effektivwerts hochfrequenter Fluktuationen beobachtet, die spannweitig stark lokalisiert scheinbar an lokalen Maxima des spannweitigen Gradienten auftreten. Die dominanten stationären QSI mit der Wellenzahl $\beta=416 \mathrm{~m}^{-1}$ besitzen an dieser Plattentiefe bereits eine Amplitude von etwa $12 \%$ der Grenzschichtrandgeschwindigkeit, siehe Abbildung 7.2.2(a).

Verfolgt man die Längswirbel anhand der Isokonturen des spannweitigen Gradienten stromab bis $x_{c} / c=0.45$, werden die angehobenen Effektivwerte hochfrequenter instationärer Fluktuationen dort teilweise gar nicht oder zumindest nicht an den gleichen spannweitigen Positionen beobachtet. Bei $x_{c} / c=0.5$ hingegen ist der Effektivwert wieder an den stromab verfolgten spannweitigen Positionen angehoben. $\mathrm{Ab} x_{c} / c=0.5$ werden dann angehobene Werte des Effektivwertes auch an spannweitigen Positionen mit lokalen Minima des spannweitigen $u_{s}$-Gradienten beobachtet. Stromab wachsen diese hochfrequenten Störungen in ihrer Amplitude an und breiten sich ausgehend von diesen Positionen spannweitig aus.

Im Folgenden sollen diese lokalisiert angehobenen Effektivwerte hochfrequenter instationärer $u_{s}$-Fluktuationen näher diskutiert werden. In Abbildung 7.2.7 ist das durch die stationären QSI deformierte zeitlich gemittelte Grenzschichtströmungsfeld bei $x_{c} / c=0.4$, bei $x_{c} / c=0.45$ und bei $x_{c} / c=0.5$ dargestellt. Dabei sind in Isokonturen die Werte des Gradienten $\frac{\partial u_{s}}{\partial z}$ dargestellt, während in Isolinien die Werte des Effektivwerts der $u_{s}$-Fluktuationen mit Frequenzen $f>1000 \mathrm{~Hz}$ dargestellt sind. Wie oben beschrieben, sind an diesen Plattentiefenpositionen keine anderen Anzeichen für den finalen Umschlag zu beobachten. Daher wird dieser angehobene Effektivwert bis $x_{c} / c=0.5$ als ein Anzeichen von Sekundärinstabilitäten und nicht als Anzeichen von turbulenten Fluktuationen interpretiert.

Es fällt auf, dass die hochfrequenten Geschwindigkeitsfluktuationen örtlich lokalisiert und überwiegend bei kleinen Wandabständen auftreten. Des Weiteren korrelieren sie räumlich gut mit hohen Beträgen des wandnormalen $u_{s^{-}}$ Gradienten. Koch et al. [54] beschreiben eine wandnahe Sekundärinstabilität, deren Ort im Strömungsfeld mit Orten großer wandnormaler Scherung korreliert (Typ III). Demnach könnte es sich bei den hier beobachteten angehobenen Effektivwerten hochfrequenter Fluktuationen um Anzeichen einer Sekundärinstabilität dieses Typs handeln. Die angehobenen Effektivwerte treten vor Erreichen der maximalen Amplitude stationärer QSI auf und lange vor anderen Anzeichen des finalen laminar-turbulenten Umschlags wie dem 
(a) $x_{c} / c=0.4$

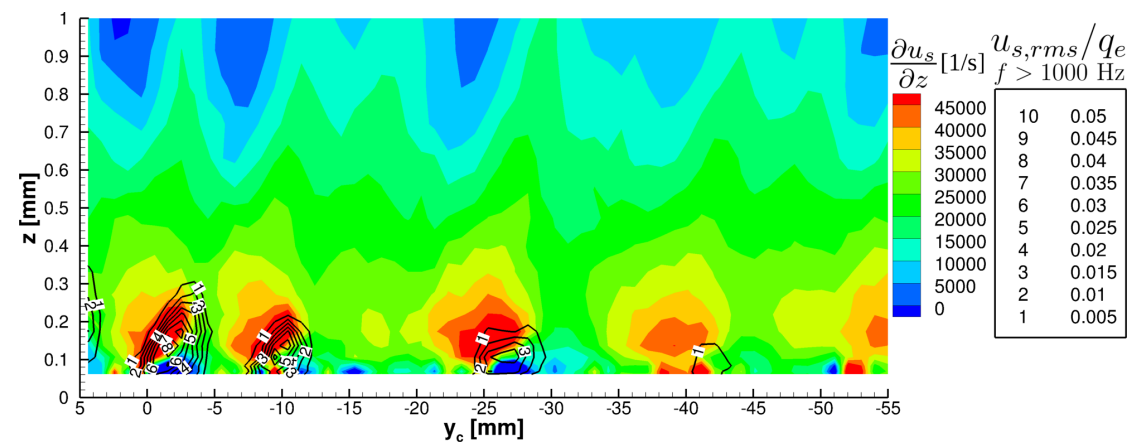

(b) $x_{c} / c=0.45$

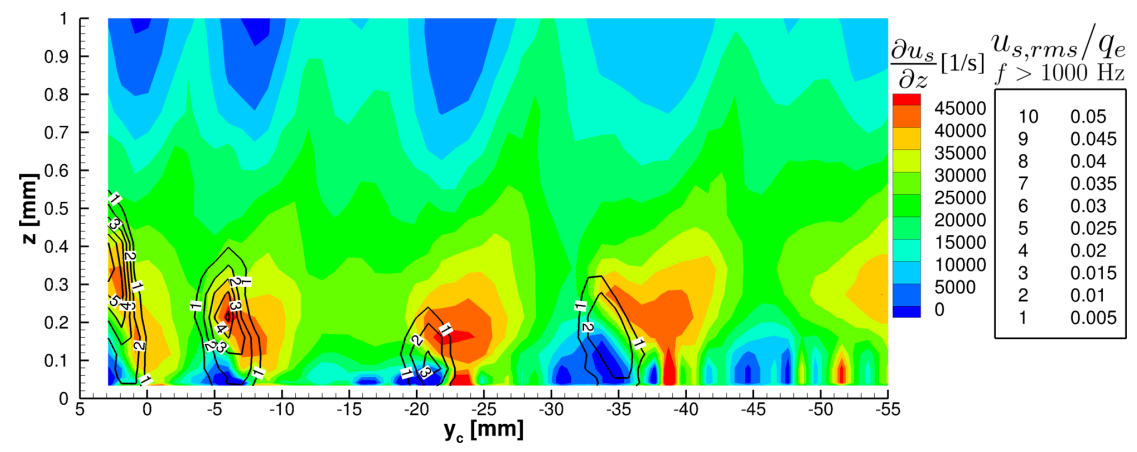

(c) $x_{c} / c=0.5$

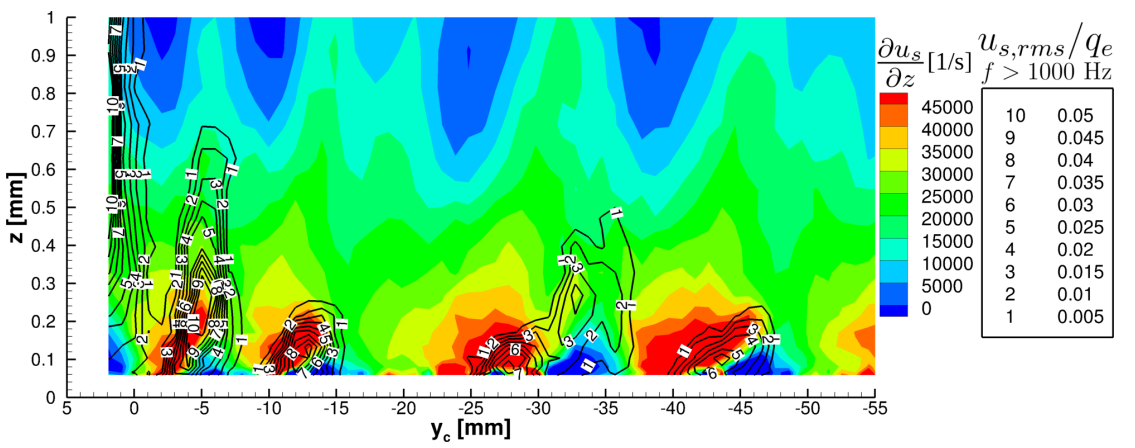

Abbildung 7.2.7: Durch stationäre QSI deformierte wandnormale $u_{s}$-Gradienten als Isokonturen und Effektivwerte $u_{s, r m s} / q_{e}$ mit einer Frequenz von $f>1000 \mathrm{~Hz}$ als Isolinien 


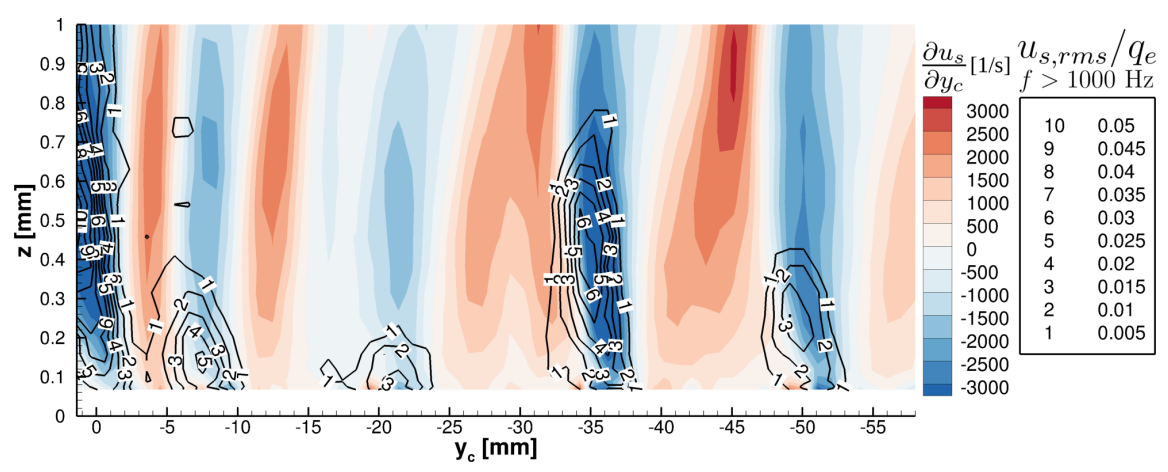

Abbildung 7.2.8: Spannweitige Gradienten $\frac{\partial u_{s}}{\partial y_{c}}$ und Effektivwerte $u_{s, r m s} / q_{e}$ mit einer Frequenz von $f>1000 \mathrm{~Hz}$ bei $x_{c} / c=0.55$

Zerfallen der stationären Längswirbel, dem Aufdicken der Grenzschicht und der Ausbreitung der hochfrequenten Geschwindigkeitsschwankungen durch das gesamte Grenzschichtströmungsfeld. Dabei ist zu beobachten, dass bei $x_{c} / c=0.45$ geringere Beträge dieses wandnormalen Gradienten auftreten als bei $x_{c} / c=0.4$ und $x_{c} / c=0.5$. Ebenso treten die Effektivwerte hochfrequenter Fluktuationen an anderen Positionen relativ zur Grenzschichtdeformation der stationären QSI auf als bei $x_{c} / c=0.4$ und $x_{c} / c=0.5$. Dies ist wie die Sprünge der Amplitudenverläufe in Abbildung 7.2.2(a) eine Konsequenz des großen zeitlichen Abstands zwischen den Messungen bei $x_{c} / c=0.4$ und $x_{c} / c=0.45$ und der veränderten Umweltbedingungen. Der Einfluss der nicht konstanten Windkanal-Reynoldszahl $R e_{K}$ beschränkt sich also wie zu erwarten nicht auf die Amplituden stationärer primärer QSI. Wie in den Abbildungen 7.2.7(a), 7.2.7(b) und 7.2.7(c) zu erkennen, verändert dieser Einfluss auch den Ort der auftretenden Sekundärinstabilitäten im stationär deformierten Grenzschichtströmungsfeld sowie ihre Amplitude. Bei $x_{c} / c=0.5$ werden bei $y_{c}=2 \mathrm{~mm}$, $y_{c}=-5 \mathrm{~mm}$ und $y_{c}=-33 \mathrm{~mm}$ auch an etwas größeren Wandabständen angehobene Effektivwerte der hochfrequenten Fluktuationen beobachtet als die Sekundärinstabilitäten vom Typ III. Die angehobenen Effektivwerte bei $y_{c}=-5 \mathrm{~mm}$ und $0.4 \mathrm{~mm}<z<0.7 \mathrm{~mm}$ werden bei $x_{c} / c=0.55$ nicht mehr an der stromab verfolgten spannweitigen Position und demselben Wandabstand beobachtet, siehe Abb. 7.2.8. Daher sind sie offensichtlich bei $x_{c} / c=0.5$ noch an den leicht höheren Wert der Windkanal-Reynoldszahl $R e_{K}$ dieser Messung geknüpft, siehe Abbildung 7.2.2(b).

In Abbildung 7.2.8 sind Werte des spannweitigen $u_{s}$-Gradienten bei $x_{c} / c=$ 0.55 in farblichen Isokonturen dargestellt, da ab dieser Plattentiefenposition 
zusätzlich zu den Sekundärinstabilitäten vom Typ III ein anderes Phänomen beobachtet wird. An einzelnen spannweitigen Positionen (insbesondere $y_{c}=1 \mathrm{~mm}$ und $y_{c}=-35 \mathrm{~mm}$ ) treten Effektivwerte hochfrequenter Fluktuationen auf, welche örtlich eher mit großen Beträgen des spannweitigen $u_{s^{-}}$ Gradienten als des wandnormalen $u_{s}$-Gradienten korrelieren. Zudem werden sie in größerem Wandabstand beobachtetet als die meisten angehobenen Effektivwerte stromauf. Beides weist auf eine Sekundärinstabilität vom Typ I hin. Die Plattentiefenposition $x_{c} / c=0.55$ ist diejenige, an der die dominanten stationären QSI ihr Amplitudenmaximum erreichen. In diesem Fall sind im Gegensatz zum Referenzfall allerdings die Beträge der negativen spannweitigen Gradienten größer als die der positiven, sodass diese Sekundärinstabilitäten an den Positionen mit negativen spannweitigen Gradienten und damit an anderer Position relativ zur jeweiligen Wirbelachse beobachtet werden. Stromab breiten sich diese hochfrequenten Geschwindigkeitsfluktuationen kontinuierlich in der Grenzschicht aus und die dominanten stationären QSI zerfallen, siehe Abbildung 7.2.6 und 7.2.2(a). Ab $x_{c} / c=0.65$ folgt das deutliche transitionelle Aufdicken der Grenzschicht.

In dem in Abschnitt 6.3 beschriebenen Referenzfall wurden hochfrequente Geschwindigkeitsfluktuationen vor allem an Orten beobachtet, an denen hohe Werte des spannweitigen Gradienten der zeitlich gemittelten $u_{s}$-Komponente gemessen wurden. Diese örtliche Korrelation wurde wie hier als Anzeichen von Sekundärinstabilitäten vom Typ I interpretiert. Im Gegensatz zum Referenzfall werden hochfrequente instationäre Fluktuationen stromab des ausgeschalteten Heizaktuators auch bereits vergleichsweise weit stromauf, näher an der Wand und örtlich korreliert mit angehobenen Werten des wandnormalen $u_{s}$-Gradienten beobachtet. Diese Beobachtungen weisen auf Sekundärinstabilitäten vom Typ III hin. Weiter stromab werden hochfrequente Fluktuationen zunehmend in größerem Wandabstand und wie im Referenzfall örtlich korreliert mit hohen Beträgen des spannweitigen $u_{s}$-Gradienten beobachtet. In kurzem Abstand stromab werden erste Anzeichen des finalen Umschlags beobachtet. Wesentlicher Unterschied zwischen der hier beobachteten Störströmung und derjenigen im Referenzfall ist die größere Wellenlänge der dominanten stationären QSI. In der Literatur ist bekannt, dass die spannweitige Wellenlänge der dominanten stationären Störungen eine große Rolle dabei spielt, welcher Typ von Sekundärinstabilität dominiert [113]. Die Wellenlänge der stationären Störungen bestimmt das Verhältnis der Geschwindigkeitsgradienten in spannweitiger und wandnormaler Richtung. 


\subsection{Erwartete Temperaturänderungen aufgrund der Wandheizung}

Ziel des Heizaktuators ist es, durch lokale Wandheizung eine spannweitig periodische Anhebung der Wandtemperatur zu erzeugen und somit die Grenzschichtströmung spannweitig periodisch zu deformieren. Aus dieser initialen Anregung soll die stationäre QSI-Kontrollmode entstehen (siehe Abschnitt 2.5.2). Parallel zu jeder Hitzdrahtmessung im $1 \mathrm{MG}$ wurde die zweidimensionale Wandtemperaturverteilung $T_{W}\left(x_{c}, y_{c}\right)$ mithilfe einer Infrarotkamera gemessen (siehe Abschnitt 5.4). Neben der beabsichtigten spannweitig periodischen Anhebung erzeugt der Heizaktuator auch eine unerwünschte, aber unvermeidbare signifikante Anhebung der spannweitig gemittelten Wandtemperatur $\bar{T}_{W}\left(x_{c}\right)$.

Zunächst soll daher im Folgenden die spannweitig gemittelte Temperaturanhebung untersucht werden (Abschnitt 7.3.1). Dabei wird vor allem geprüft, welchen Einfluss diese Anhebung als direkte Wirkung auf Hitzdrahtmessungen in der Grenzschicht stromab hat. Im Anschluss wird die erzeugte spannweitige Wandtemperaturperiodizität abhängig von der Heizleistung untersucht und deren Amplitude als physikalisch relevantes Maß für die Anregungsstärke eingeführt (Abschnitt 7.3.2). Die erwartete Wirkung der periodisch angehobenen Wandtemperatur auf Hitzdrahtmessungen wird danach diskutiert (Abschnitt 7.3.3). Zuletzt wird eine indirekte Wirkung der angehobenen spannweitig gemittelten Wandtemperatur diskutiert: Die erwartete Destabilisierung der Grenzschicht (Abschnitt 7.3.4).

\subsubsection{Anhebung der spannweitig gemittelten Wandtemperatur}

An den Heizelementen wird durch diffusiven Wärmetransport Wärme seitlich und vertikal im Modell durch die verschiedenen verwendeten Materialien geleitet. Die über die Modelloberfläche strömende Grenzschichtströmung transportiert konvektiv Wärme ab. Diese Prozesse tendieren gemeinsam zu einem Gleichgewichtszustand, in dem bei konstanter Heizleistung $P$ eine stationäre Temperaturverteilung an der Modelloberfläche erreicht wird. In den Messungen im Rahmen dieser Arbeit wurden stets solche stationären Zustände untersucht. Dazu wurde bei jeder Änderung der Heizleistung eine Wartedauer beachtet und währenddessen mithilfe der Infrarotkamera überprüft, dass 
keine signifikanten zeitlichen Änderungen der Wandtemperaturverteilung mehr auftraten.

Zusätzlich zum erwünschten Effekt einer spannweitigen Wandtemperaturperiodizität mit der Wellenlänge der stationären Kontrollmode bewirkt die Heizung auch eine Anhebung der spannweitig gemittelten Wandtemperatur, besonders an der $x_{c}$-Position der jeweils betriebenen Heizreihe. Diese Anhebung hat einen Einfluss auf die Form des spannweitig gemittelten Grenzschichtprofils und damit auf die Stabilitätseigenschaften der Grenzschicht. Da Hitzdrahtmessungen stark temperaturabhängig sind, beeinflusst eine Temperaturanhebung in der Grenzschicht auch die Hauptmessmethode. Um diese Effekte zu untersuchen und zu quantifizieren, wurden parallel zu den Hitzdrahtmessungen zweidimensionale Wandtemperaturverteilungen $T_{W}\left(x_{c}, y_{c}\right)$ durch Infrarot-Thermografie gemessen (siehe auch Abschnitt 5.4). Dabei wurde lediglich der Plattentiefenbereich des Naseneinsatzes (bis $x_{c} / c=0.25$ ) ausgewertet. Das dort als Oberflächenabschluss verwendete Epoxidharz eignet sich durch seine hohe Emissivität für Infrarotmessungen der Wandtemperatur. Bei den Profiltiefen $x_{c} / c>0.25$ besteht das Modell aus Aluminium, was eine verlässliche Messung der Wandtemperatur mit dieser Methode unmöglich macht. Innerhalb des fokussierten spannweitigen Bereichs wurde die Wandtemperaturverteilung spannweitig gemittelt. Je nach verwendeter Heizreihe und -leistung ergibt sich ein anderer Verlauf dieser gemittelten Wandtemperatur $\bar{T}_{W}\left(x_{c}\right)$.

Für die Untersuchungen zur künstlichen Anregung der QSI-Kontrollmode mit systematischer Variation der Heizleistung in Abschnitt 7.4 wurde als höchste Heizleistung jeweils $P=14 \mathrm{~W}$ untersucht. Die spannweitig gemittelte Wandtemperaturverteilung $\bar{T}_{W}\left(x_{c}\right)$ ist für die acht jeweils einzeln betriebenen Heizreihen bei dieser höchsten Heizleistung in Abbildung 7.3.1 dargestellt. Dabei wurde die spannweitig gemittelte Wandtemperatur im ungeheizten Fall $\bar{T}_{W, 0}$ abgezogen. Die Ausdehnung der jeweiligen Heizreihe in $x_{c}$-Richtung ist durch vertikale Linien derselben Farbe eingezeichnet. In Abschnitt 4.5.1 wurde beschrieben, dass die ungeradzahlig nummerierten Heizreihen aus langen Heizelementen bestehen und die geradzahlig nummerierten Reihen aus kurzen. Dieser Unterschied wurde in Abbildung 7.3.1 mit durchgezogenen und gestrichelten Linien für den jeweiligen Heizreihentyp hervorgehoben. Dabei fällt der Unterschied auf, dass Heizreihen mit kurzen Elementen eine höhere maximale spannweitig gemittelte Wandtemperaturanhebung erzeugen als benachbarte Heizreihen mit langen Elementen bei gleicher Heizleistung. Dieselbe Heizleistung bewirkt bei kurzen Heizelementen eine höhere Wärme- 


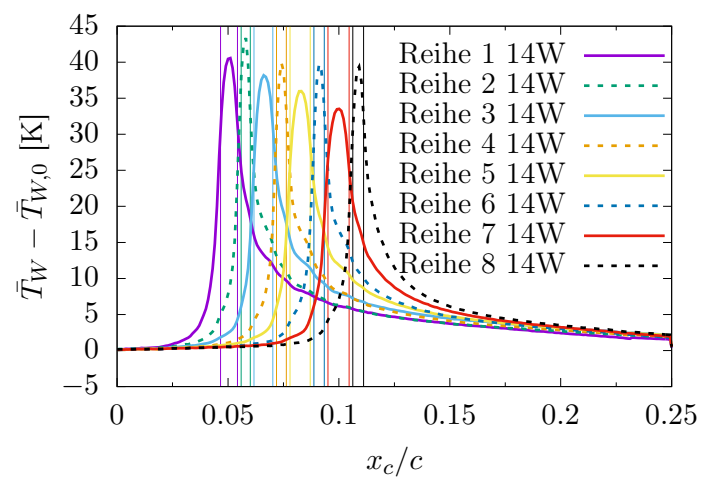

Abbildung 7.3.1: Spannweitig gemittelte Wandtemperaturverteilung bei der Heizleistung $P=14 \mathrm{~W}$ abhängig von der Profiltiefe $x_{c}$

stromdichte als bei langen Heizelementen und führt damit zu einer stärkeren Wandtemperaturanhebung. Zudem ist zu beobachten, dass das jeweilige Maximum der spannweitig gemittelten Anhebung mit zunehmender Profiltiefe $x_{c}$ entlang Heizreihen gleichen Typs abnimmt. Dies liegt daran, dass der konvektive Wärmeabtransport durch die beschleunigte Grenzschichtströmung stromab der Staulinienposition $x_{c} / c=0.013$ zunimmt.

Um den Einfluss der spannweitig gemittelten Anhebung der Wandtemperatur auf Hitzdrahtmessungen in der stromab entwickelten Grenzschicht und später auch auf die Stabilitätseigenschaften dieser Grenzschicht abzuschätzen, wurden mithilfe von COCO (siehe Abschnitt 3.1) Grenzschichtlösungen bestimmt, bei denen die in Abbildung 7.3.1 dargestellten spannweitig gemittelten Wandtemperaturverteilungen als isotherme Randbedingung an der Wand vorgegeben wurden. Dabei wurde bzgl. der Wandtemperatur für $x_{c} / c>0.25$ die Annahme getroffen, dass die hohe Wärmeleitfähigkeit des Aluminiums Wärme hinreichend schnell abführt, sodass die Wandtemperatur sich dort nicht von derjenigen im ungeheizten Fall unterscheidet. Die numerische Grenzschichtlösung zeigt für den ungeheizten Fall, dass die Wandtemperatur entlang der Profiltiefe der Modellplatte nur um $0.04 \mathrm{~K}$ variiert. Um einen Eindruck von der beeinflussten Temperaturgrenzschicht zu vermitteln, sind in Abbildung 7.3.2 numerische wandnormale Temperaturprofile exemplarisch aus der Grenzschichtlösung für die bei $P=14 \mathrm{~W}$ betriebene Heizreihe 1 (mittlere Plattentiefenposition $x_{c} / c=0.05$ ) dargestellt. Dabei sieht man, dass an der mittleren Profiltiefenposition der Anregung (bzw. der Heizelemente) die Temperatur in der Grenzschicht mit dem Wandabstand rasch von dem Wert an der Wand zum Wert der Temperatur am Grenzschichtrand abnimmt. Die 


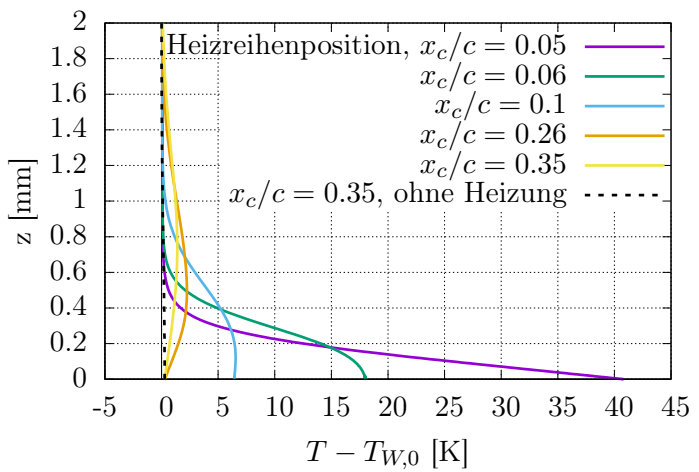

Abbildung 7.3.2: Wandnormale Temperaturprofile der Grenzschichtlösung für den Fall mit der experimentell bestimmten Wandtemperaturverteilung bei Betrieb der Heizreihe 1 mit der Heizleistung $P=14 W$

Temperaturgrenzschicht hat also anfangs eine geringe Dicke. Durch diffusiven Wärmetransport in wandnormaler Richtung dickt sie stromab rasch auf. Der maximale Betrag der Temperatur in der Grenzschicht nimmt parallel dazu mit $x_{c}$ ab. Bereits in dem Profiltiefenbereich, für den Messwerte der Wandtemperatur vorliegen, nimmt diese aber schneller mit $x_{c}$ ab als die maximale Temperaturanhebung in der Grenzschicht darüber. Bereits ab etwa $x_{c} / c=0.07$ wird die Grenzschicht also an der Wand geringfügig gekühlt und ein Teil der zugeführten Wärmemenge der Grenzschicht wieder entzogen. Dieser Kühlungseffekt wird ab $x_{c} / c=0.25$ wegen der als geringer angenommenen Temperatur der Aluminiumoberfläche noch einmal stärker. Mit den Temperaturprofilen $T\left(x_{c}, z\right)$ aus den numerischen Grenzschichtlösungen und insbesondere den Gradienten $\partial T\left(x_{c}, z\right) / \partial z$ an der Wand lässt sich die Wärmestromdichte $\dot{q}$ in die Grenzschicht hinein anhand des Fourierschen Gesetzes bestimmen:

$$
\dot{q}=-\left.\Lambda \frac{\partial T\left(x_{c}, z\right)}{\partial z}\right|_{z=0}
$$

Dabei steht $\Lambda=0.0261 \mathrm{~W} /(\mathrm{m} \cdot \mathrm{K})$ für die Wärmeleitfähigkeit von Luft. Der gesamte Wärmestrom, der auf der Fläche des Heizaktuator-Naseneinsatzes der Grenzschicht zugeführt wird, entspricht dann mit der Breite $b=300 \mathrm{~mm}$ der Heizreihe:

$$
\dot{Q}=-\left.\int_{x_{c} / c=0}^{0.25} \Lambda b \frac{\partial T\left(x_{c}, z\right)}{\partial z}\right|_{z=0} d x_{c}
$$

Der Wärmestrom in die Grenzschicht für den exemplarischen Fall, in dem Heizreihe 1 mit $P=14 \mathrm{~W}$ betrieben wurde, hat einen Wert von $\dot{Q}=7.94 \mathrm{~W}$. 
(a)

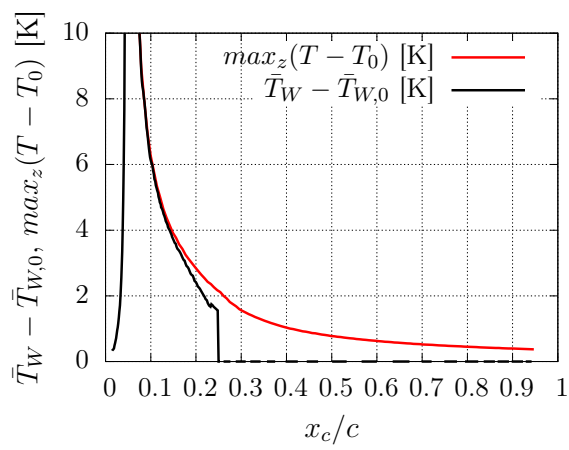

(b)

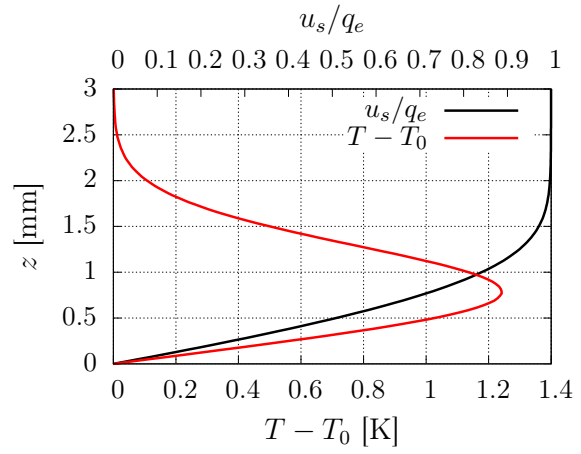

Abbildung 7.3.3: (a) Exemplarischer Verlauf der wandnormal maximalen Temperaturanhebung in der Grenzschicht $\max _{z}(\Delta T)$ und der Anhebung der spannweitig gemittelten Wandtemperatur entlang der Plattentiefe (b) Wandnormales Profil der Temperaturanhebung $\Delta T$ bei $x_{c} / c=0.35$ für die mit $P=14 W$ betriebene Heizreihe 1 gegenüber dem unbeheizten Fall

Demnach wird bis $x_{c} / c=0.25$ die Differenz $6.06 \mathrm{~W}$ durch das Modell abgeführt.

Bei Plattentiefenpositionen im Bereich der Aluminiumoberfläche, an denen die Wandtemperatur sich gemäß der oben getroffenen Annahme nicht mehr vom ungeheizten Fall unterscheidet, verbleibt eine Temperaturerhöhung $\Delta T(z)=T(z)-T_{0}(z)$ in der Grenzschicht, so auch am Ort der in Abschnitt 7.4 folgenden Untersuchungen zur systematischen Variation der Heizleistung $\left(x_{c} / c=0.35\right)$. Dabei bezeichnet $T_{0}(z)$ das wandnormale Temperaturprofil im ungeheizten Fall. Betrachtet man an dieser Plattentiefenposition die Differenz $\Delta T(z)$ zwischen dem numerischen Temperaturgrenzschichtprofil im maximal geheizten und ungeheizten Fall, erhält man im wandnormalen Maximum Werte zwischen $1.2 \mathrm{~K}<\max _{z}(\Delta T)<1.3 \mathrm{~K}$, je nach verwendeter Heizreihe. Beispielhaft ist der Verlauf der wandnormal maximalen Temperaturanhebung $\max _{z}(\Delta T)$ in der Grenzschicht stromab der maximal geheizten Heizreihe 1 im Vergleich zur Anhebung der spannweitig gemittelten Wandtemperatur $\Delta T_{W}=\bar{T}_{W}-\bar{T}_{W, 0}$ in Abbildung 7.3.3(a) dargestellt. Das wandnormale Profil der Differenztemperatur $\Delta T(z)$ bei $x_{c} / c=0.35$ aus der zugehörigen numerischen Grenzschichtlösung ist in Abbildung 7.3.3(b) dargestellt. Zur besseren Einordnung in die Grenzschicht ist das $u_{s}$-Profil mit eingezeichnet. Die numerisch bestimmten maximalen Temperaturanhebungen 
bei $x_{c} / c=0.35$ entsprechen gemäß der in Abschnitt 5.1.1 beschriebenen Temperaturkompensation für Hitzdrahtmessungen (Gleichung 5.1.21) bei Annahme üblicher Werte für die Drahttemperatur einer maximalen Änderung der Anemometerspannung $E$ um $0.28 \%$. Durch die nichtlineare Eigenschaft des Kingschen Gesetzes (Gleichung 5.1.20) bewirkt eine geringe Änderung der Anemometerspannung im Bereich hoher Anemometerspannungen bzw. hoher Geschwindigkeitsbeträge eine größere Änderung des ausgewerteten Geschwindigkeitsbetrags $U$ als im Bereich niedriger Anemometerspannungen.

Berücksichtigt man diesen Einfluss, lässt sich mit einem repräsentativen kalibrierten Zusammenhang $U(E)$ und dem bekannten Grenzschichtprofil $U(z)$ aus der COCO-Grenzschichtlösung der zu erwartende Geschwindigkeitsfehler $\Delta U(z)$ aufgrund der spannweitig gemittelten Temperaturanhebung abschätzen. Dieser Fehler erreicht bei $x_{c} / c=0.35$ in seinem wandnormalen Maximum den Wert $\Delta U=0.43 \mathrm{~m} / \mathrm{s}$, was $1.7 \%$ der Grenzschichtrandgeschwindigkeit $q_{e}$ an dieser Position entspricht. Dieser Fehler ist größer als der zu erwartende methodische Fehler von Hitzdrahtmessungen, wie er in Abschnitt 5.1.6 bestimmt wurde und muss bei der Interpretation der Ergebnisse berücksichtigt werden. Für die Bestimmung der Amplitude der angeregten QSI-Kontrollmode ist allerdings der Einfluss der spannweitig periodischen Temperaturanhebung wichtiger, welcher im folgenden Abschnitt abgeschätzt wird.

\subsubsection{Erzeugte Wandtemperaturperiodizität}

Wie bereits in Abschnitt 5.4 beschrieben wurde, reichte die optische Aufösung der Infrarotmessungen parallel zu den Hitzdrahtmessungen im 1MG nicht aus, um die Amplitude der spannweitigen Wandtemperaturperiodizität $\hat{T}_{W}$ zu bestimmen. Daher wurde der Heizaktuator-Naseneinsatz separat in einem Testwindkanal mit kleineren Düsenabmessungen mit derselben Anströmgeschwindigkeit, demselben geometrischen Schiebewinkel und ähnlicher Anströmtemperatur wie bei den Grenzschichtmessungen im 1MG untersucht. $\mathrm{Zu}$ jeder in den Grenzschichtmessungen verwendeten Heizreihe und Heizleistung wurde die zweidimensionale Wandtemperaturverteilung $T_{W}\left(x_{c}, y_{c}\right)$ der Modelloberfläche mithilfe einer Infrarotkamera aus einem geringeren Abstand als am 1MG gemessen, sodass mit der realisierten räumlichen Auflösung auch die spannweitige Periodizität mitsamt ihrer Amplitude $\hat{T}_{W}$ aufgelöst werden konnte. 


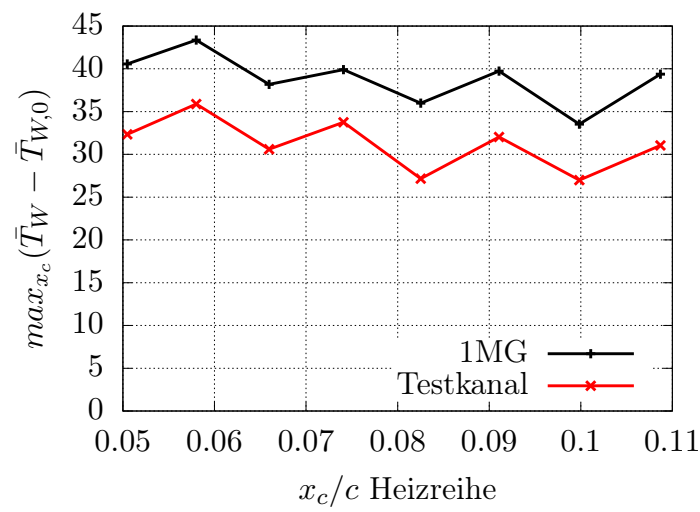

Abbildung 7.3.4: Vergleich der spannweitig gemittelten Wandtemperaturanhebung im 1MG und im Testwindkanal abhängig von der mittleren Profiltiefenposition der jeweiligen Heizreihe bei der Leistung $P=14 \mathrm{~W}$

Da im Testwindkanal nur der Naseneinsatz ohne die restliche Modellplatte und ohne Verdrängungskörper darüber untersucht wurde, entspricht die Grenzschichtströmung an der Modelloberfläche nicht derjenigen während der Grenzschichtmessungen im 1MG. Es ist zu erwarten, dass die veränderte Druckverteilung und Strömungsrichtung sowie andere Geschwindigkeitsbeträge in der Grenzschicht an der untersuchten Oberfläche zu einem veränderten thermischen Gleichgewicht bei gleicher Heizleistung führen. Um die Übertragbarkeit der Ergebnisse aus dem Testkanal auf die Messungen im 1MG zu bewerten, wurde auch aus den Messungen im Testwindkanal die Anhebung der spannweitig gemittelten Wandtemperatur $\bar{T}_{W}-\bar{T}_{W, 0}$ ausgewertet. Dabei bezeichnet $\bar{T}_{W}$ den spannweitigen Mittelwert der Wandtemperatur und der Index 0 bezeichnet den Fall ohne künstliche Heizung. Für die jeweils maximal untersuchte Heizleistung $P=14 \mathrm{~W}$ pro einzeln betriebener Heizreihe wurde der maximale Wert dieser Anhebung in Profiltiefenrichtung mit demjenigen im 1MG verglichen, siehe Abbildung 7.3.4. Dabei ist die maximale Temperaturanhebung abhängig von der mittleren Profiltiefenposition der jeweiligen Heizreihe dargestellt. In beiden Windkanälen ergibt sich ein qualitativ ähnlicher Verlauf entlang der Heizreihenposition $x_{c}$, wobei im Testwindkanal systematisch leicht geringere Temperaturanhebungen beobachtet wurden. Die gemittelte Differenz der spannweitig gemittelten Anhebungen entspricht $7 \mathrm{~K}$ oder etwa $20 \%$ des jeweiligen Wertes im 1MG. Trotz dieser Abweichung wird im Folgenden angenommen, dass die im Testwindkanal bestimmten Amplituden der spannweitigen Wandtemperaturperiodizität repräsentativ für die tatsächlichen Amplituden im 1MG verwendet werden können. 


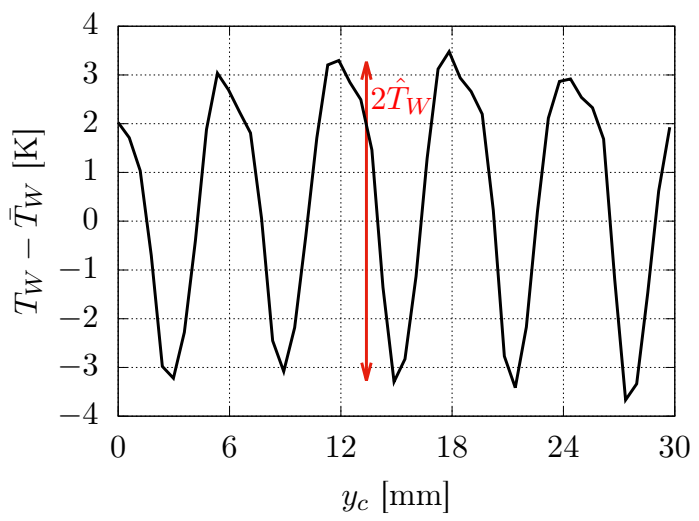

Abbildung 7.3.5: Exemplarische, im Testkanal gemessene spannweitig periodische Wandtemperaturverteilung auf der Heizaktuatoroberfläche (Heizreihe $1, P=14 \mathrm{~W}$ )

Beispielhaft ist ein im Testwindkanal beobachteter spannweitiger Wandtemperaturverlauf in Abbildung 7.3.5 dargestellt. Dieser Verlauf hat nahezu die Form einer harmonischen Periodizität mit der Wellenlänge $\lambda=6 \mathrm{~mm}$ und der Amplitude $\hat{T}_{W}$ um den spannweitigen Mittelwert $\bar{T}_{W}$. Für jede Heizreihe und jede Heizleistung wurde an jeder Profiltiefenposition ein solcher spannweitiger Bereich mit einer spannweitigen Länge von $5 \lambda$ mit $\lambda=6 \mathrm{~mm}$ im scharf abgebildeten Bereich ausgewählt. Die Amplitude $\hat{T}_{W}$ wurde jeweils aus dem Effektivwert der Abweichung der $Z$ Einzelmesswerte $T_{W, i}$ vom spannweitigen Mittelwert in diesem Bereich wie folgt ausgewertet:

$$
\hat{T}_{W}\left(x_{c}\right)=\sqrt{2} \sqrt{\frac{1}{Z} \sum_{i=1}^{Z}\left(T_{W, i}\left(x_{c}, y_{c}\right)-\bar{T}_{W}\left(x_{c}\right)\right)^{2}}
$$

Aus den ausgewerteten Amplituden $\hat{T}_{W}\left(x_{c}\right)$ wird im Folgenden für jede Heizreihe und Heizleistung die maximale Amplitude $\max _{x_{c}}\left(\hat{T}_{W}\left(x_{c}\right)\right)$ als die erzeugte Amplitude der Wandtemperaturperiodizität ausgewählt und vereinfachend schlicht als $\hat{T}_{W}$ bezeichnet. Ihre Abhängigkeit von der Heizleistung ist in Abbildung 7.3.6 je Heizreihe dargestellt. Für alle Heizreihen wird ein klarer linearer Zusammenhang zwischen der Heizleistung und $\hat{T}_{W}$ beobachtet. Mithilfe eines Verfahrens zur Minimierung der Summe der Fehlerquadrate wurden an diese Verläufe Ausgleichsgeraden angepasst, welche bei der Diskussion der Grenzschichtexperimente zur Zuordnung zwischen der elektrischen Heizleistung und $\hat{T}_{W}$ verwendet werden. 


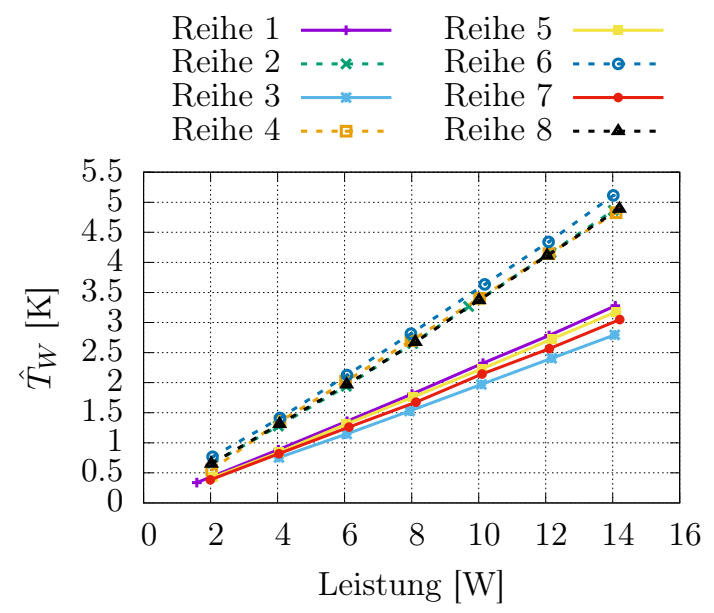

Abbildung 7.3.6: Abhängigkeit der von jeder Heizreihe erzeugten, entlang der Profiltiefe $x_{c}$ maximalen Amplitude der spannweitigen Wandtemperaturperiodizität von der Heizleistung

Vergleicht man diese Zusammenhänge für die zwei unterschiedlichen Geometrien von Heizreihen (für die Beschreibung der Geometrien siehe Abschnitt 4.5.1), fällt auf, dass $\hat{T}_{W}$ für Heizreihen mit kurzen Heizelementen (geradzahlig) bei gleicher Heizleistung stets höher ist als für Heizreihen mit langen Elementen (ungeradzahlig). Zudem wächst sie auch steiler linear mit der Heizleistung. Durch die geringere Länge führt dieselbe Heizleistung bei kurzen Heizelementen zu einer höheren Wärmestromdichte, was die höhere Amplitude der Wandtemperaturperiodizität erklärt. Um dieselbe Amplitude $\hat{T}_{W}$ zu erzeugen, ist mit langen Heizelementen im Mittel die 1.6-fache Leistung nötig.

Für die später folgende Auswertung und Diskussion der Grenzschichtmessungen stromab der Heizanregung ist zusätzlich die Frage von Bedeutung, ob ggf. die Phasenlage der Wandtemperaturperiodizität von der Heizleistung abhängt. Dazu ist exemplarisch der spannweitige Verlauf der Wandtemperatur für Heizreihe 2 (bei $x_{c} / c=0.058$ ) bei drei ausgewählten Heizleistungen in Abbildung 7.3.7(a) dargestellt. Wie zu erwarten, wird keine Abhängigkeit der Phasenlage von der Heizleistung beobachtet. Dies gilt auch für die anderen Heizreihen.

Ein weiteres Ergebnis der Messungen im Testwindkanal ist, dass die erzeugte Amplitude der spannweitigen Wandtemperaturperiodizität $\hat{T}_{W}$ bei den meis- 
(a)

$14 \mathrm{~W}$

$8 \mathrm{~W}$

$4 \mathrm{~W}$

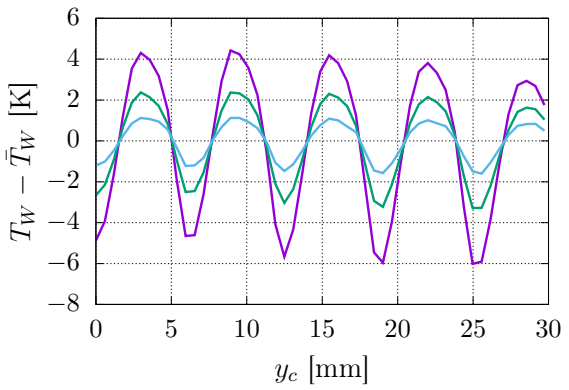

(b)

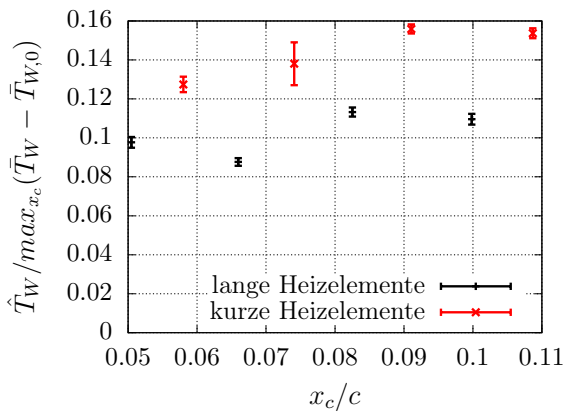

Abbildung 7.3.7: (a) Exemplarischer spannweitiger Wandtemperaturverlauf für Heizreihe $2\left(x_{c} / c=0.058\right)$ bei verschiedenen ausgewählten Heizleistungen (b) Verhältnis der Amplitude der spannweitigen Wandtemperaturperiodizität und der bzgl. $x_{c}$ maximalen spannweitig gemittelten Wandtemperaturanhebung

ten Heizreihen in einem bzgl. der Heizleistung nahezu konstanten Verhältnis zur spannweitig gemittelten und in Plattentiefenrichtung maximalen Anhebung der Wandtemperatur $\max _{x_{c}}\left(\bar{T}_{W}-\bar{T}_{W, 0}\right)$ (ebenfalls aus Messungen am Testkanal bestimmt) steht. Des Weiteren hat dieses Verhältnis für Heizreihen gleicher Geometrie einen sehr ähnlichen Wert. Das Verhältnis ist für die verschiedenen Heizreihen abhängig von ihrer jeweiligen mittleren Plattentiefenposition in Abbildung 7.3.7(b) dargestellt. Der dargestellte Fehlerbalken repräsentiert die doppelte Standardabweichung des Verhältnisses bzgl. der Leistungsvariation je Heizreihe. Das Verhältnis $\hat{T}_{W} / \max _{x_{c}}\left(\bar{T}_{W}-\bar{T}_{W, 0}\right)$ ist für Heizreihen mit kurzen Heizelementen stets größer als für Heizreihen mit langen Heizelementen. Dieselbe Amplitude der spannweitigen Wandtemperaturperiodizität kann also mit kurzen Elementen bei geringerer unerwünschter Anhebung der spannweitig gemittelten Wandtemperatur erreicht werden.

\subsubsection{Einfluss der Wandtemperaturperiodizität auf Hitzdrahtmessungen}

Der Einfluss der spannweitig gemittelten Wandtemperaturanhebung auf Hitzdrahtmessungen wurde in Abschnitt 7.3.1 abgeschätzt. Diesem überlagert ist der Einfluss der eigentlich beabsichtigten spannweitigen Wandtemperaturperiodizität mit der Amplitude $\hat{T}_{W}$, welche stromab eine spannweitig periodische 


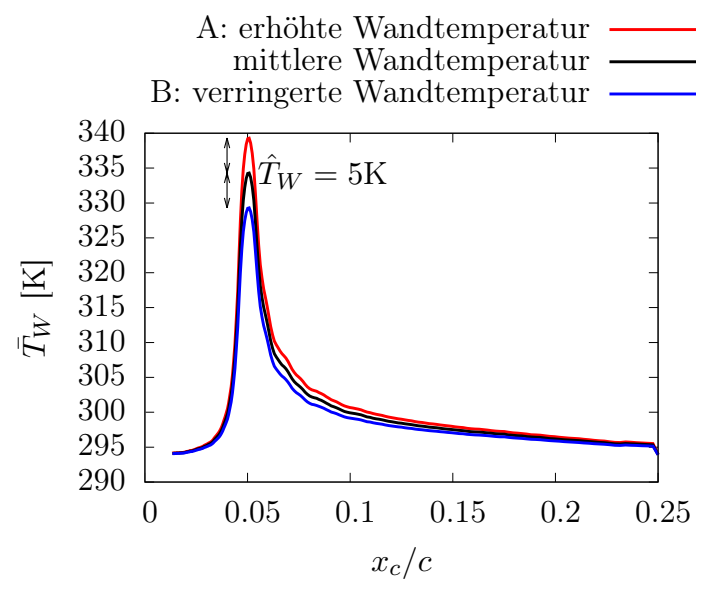

Abbildung 7.3.8: Skalierung der gemessenen spannweitig gemittelten Wandtemperaturverteilung auf die Extrema der spannweitigen Wandtemperaturperiodizität

Temperaturanhebung in der Grenzschicht bewirkt. Wie in Abbildung 7.3.6 dargestellt wurde, erreicht die Amplitude der Wandtemperaturperiodizität bei manchen Heizreihen und maximaler Heizleistung Werte von etwa $\hat{T}_{W} \approx 5 \mathrm{~K}$. Um den Einfluss dieser Periodizität auf die spannweitige Temperaturperiodizität in der stromab gelegenen Grenzschicht vereinfacht abzuschätzen, wurden die gemessenen spannweitig gemittelten Wandtemperaturverteilungen so linear skaliert, dass der Spitzenwert der Temperaturerhöhung jeweils $5 \mathrm{~K}$ über (Fall A) bzw. $5 \mathrm{~K}$ unter (Fall B) dem spannweitigen Mittelwert $\max _{x_{c}}\left(\bar{T}_{W}\right)$ der jeweils betrachteten Heizreihe liegt. Zur Veranschaulichung ist in Abb. 7.3.8 die im 1MG gemessene spannweitig gemittelte Wandtemperaturverteilung exemplarisch für die bei maximaler Heizleistung betriebene Reihe $1 \mathrm{im}$ Vergleich zu den beiden skalierten Wandtemperaturverteilungen A und B dargestellt.

Diese Skalierung auf Fälle A und B wurde für jede bei maximaler Heizleistung $P=14 \mathrm{~W}$ betriebene Heizreihe durchgeführt. Für beide Fälle wurde jeweils eine weitere Grenzschichtlösung mit COCO bestimmt. Vergleicht man nun die Grenzschichtlösung für Fall A mit der Grenzschichtlösung für Fall B, lassen sich Temperaturdifferenzprofile $\Delta T\left(x_{c}, z\right)=T_{A}\left(x_{c}, z\right)-T_{B}\left(x_{c}, z\right)$ bilden. Im Folgenden wird angenommen, dass diese Temperaturdifferenz eine Abschätzung für die spannweitig periodischen Temperaturunterschiede zwischen den spannweitigen Orten größter und den Orten geringster Temperaturanhebung darstellen. Da die Grenzschichtlösungen spannweitig homogene Zustände 


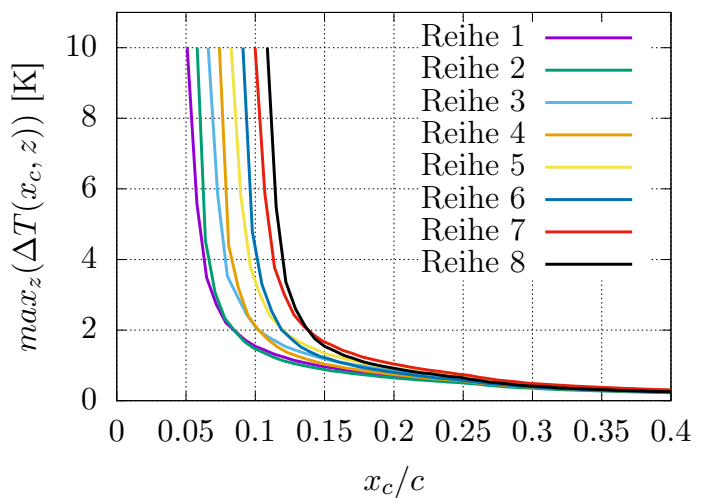

Abbildung 7.3.9: Wandnormal maximale Temperaturdifferenz jeweils zwischen zwei Grenzschichtlösungen (Fälle A und B), die vereinfachend als Zustand am jeweiligen spannweitigen Extremwert der eigentlich spannweitig periodisch beeinflussten Grenzschicht interpretiert werden

beschreiben, wird ein Ausgleich der spannweitigen Temperaturunterschiede nicht berücksichtigt. In der Realität wird ein solcher Temperaturausgleich sowohl diffusiv als auch konvektiv durch die stationären Längswirbel stattfinden. Im Folgenden werden die so bestimmten Temperaturunterschiede benutzt, um den Einfluss auf Hitzdrahtmessungen abzuschätzen. Wegen der Vernachlässigung des spannweitigen Temperaturausgleichs handelt es sich um eine obere Abschätzung für den zu erwartenden Einfluss.

Die wandnormalen Maxima der auf diese Art abgeschätzten Temperaturunterschiede sind je Heizreihe abhängig von der Plattentiefenposition $x_{c} / c$ in Abbildung 7.3.9 dargestellt. Wie die Temperaturanhebung in der jeweiligen Grenzschichtlösung selbst, nimmt die Temperaturdifferenz der beiden Grenzschichtlösungen für Fall $\mathrm{A}$ und $\mathrm{B}$ rasch mit $x_{c}$ ab. Im wandnormalen Maximum erhält man bei $x_{c} / c=0.35$ für alle maximal geheizten Reihen einen Temperaturunterschied in der Grenzschicht von $0.27 \mathrm{~K}<\max _{z}(\Delta T)<0.37 \mathrm{~K}$, abhängig von der verwendeten Heizreihe. Bei typischen Werten für die Drahttemperatur und die Fluidtemperatur bei der Kalibrierung entspricht ein Temperaturunterschied von $0.37 \mathrm{~K}$ gemäß Gleichung (5.1.21) einer maximalen Änderung der Anemometerspannung $E$ um $0.05 \%$. Wie bei der Abschätzung des spannweitig gemittelten Geschwindigkeitsfehlers hängt der erwartete spannweitig periodische Geschwindigkeitsfehler vom Betrag des spannweitig gemittelten Geschwindigkeitsbetrags $U(z)$ in der Grenzschicht und damit vom Wandabstand ab. Bei Anwendung eines repräsentativ kalibrierten Zusam- 
menhangs $U(E)$ und des bekannten Grenzschichtprofils $U(z)$ aus der COCOGrenzschichtlösung lässt sich der maximal erwartete Geschwindigkeitsfehler aufgrund spannweitig periodischer Temperaturunterschiede auf $0.27 \%$ der Grenzschichtrandgeschwindigkeit $q_{e}$ abschätzen. Der Wandabstand, an dem dieser maximale Fehler erwartet wird, ist sehr ähnlich zu dem Wandabstand, an dem in Abschnitt 7.4 die Amplitude $\hat{u}_{s} / q_{e}$ der Kontrollmode ausgewertet wird. Daher stellt dieser Fehler tatsächlich auch eine obere Abschätzung für den temperaturbedingten Fehler der gemessenen Amplitude der Kontrollmode bei der höchsten Heizleistung dar. Ob aufgrund dieses Fehlers eine zu große oder zu kleine Amplitude gemessen wird, hängt zusätzlich von der spannweitigen Phasenlage der Temperaturperiodizität relativ zur Phasenlage der Kontrollmode ab.

Zumindest bei den in Abschnitt 7.4 beschriebenen Untersuchungen bei $x_{c} / c=0.35$ zur Instabilitätsamplitude bei systematischer Variation der Heizleistung kann der erwartete spannweitig periodische Einfluss auf die Geschwindigkeitsmessung mit Hitzdrahtsonden als gering bewertet werden. In Abschnitt 7.5 werden aber auch Messungen an weiter stromauf gelegenen Positionen $\left(x_{c} / c \geq 0.15\right)$ diskutiert. Dort besteht nach dieser Abschätzung die Gefahr, dass spannweitig periodische Temperaturunterschiede als spannweitig periodische Geschwindigkeitsunterschiede und damit als eine Grenzschichtdeformation durch stationäre QSI fehlinterpretiert werden. Diese spannweitige Periodizität hätte dieselbe Wellenlänge $\lambda=6 \mathrm{~mm}$ wie die stationäre QSI-Kontrollmode. Letztere ist allerdings stromab von $x_{c} / c=0.05$ linear durch die instabile Grenzschicht angefacht. Man erwartet also stark mit $x_{c}$ wachsende Amplituden, während die erwarteten spannweitig periodischen Temperaturunterschiede rasch mit $x_{c}$ abnehmen. Dies bedeutet, dass das Amplitudenwachstum der Kontrollmode im vorderen Bereich der Modellplatte bei ungünstiger Phasenlage womöglich aufgrund der Temperaturunterschiede nicht korrekt gemessen wird. Es kann aber ausgeschlossen werden, dass die spannweitigen Temperaturunterschiede allein als angefachte QSI-Mode fehlinterpretiert werden, ohne dass eine solche angeregt wurde.

\subsubsection{Einfluss der spannweitig gemittelten Heizung auf die lineare Stabilität}

Neben der möglichen Beeinflussung der temperatursensitiven Messmethode hat die Anhebung der spannweitig gemittelten Wandtemperatur auch einen Einfluss auf die Stabilitätseigenschaften der Grenzschicht. Daher wurden 
an den im vorherigen Abschnitt beschriebenen Grenzschichtlösungen, in denen die am $1 \mathrm{MG}$ gemessenen spannweitig gemittelten Wandtemperaturverteilungen $\bar{T}_{W}\left(x_{c}\right)$ als Randbedingung berücksichtigt wurden, lineare lokale Stabilitätsuntersuchungen mit der Software NOLOT (siehe Abschnitt 3.2) durchgeführt. Die Ergebnisse werden im Folgenden mit denen für den ungeheizten Fall verglichen.

\section{Stationäre QSI}

Für die künstliche Anregung der stationären Kontrollmode ist es besonders von Interesse, inwiefern die Anfachung dieser Mode durch die spannweitig gemittelte Anhebung der Grenzschichttemperatur beeinflusst wird. Es soll gezeigt werden, dass erhöhte Amplituden der Kontrollmode tatsächlich die Konsequenz der spannweitig periodischen Anregung durch die Wandheizung sind und nicht im Wesentlichen eine Konsequenz der destabilisierenden Wirkung der spannweitig mittleren Heizung.

Neben der Kontrollmode sind für das beeinflusste Transitionsszenario aber auch andere stationäre QSI-Moden von Bedeutung. In Abbildung 7.3.10(a) sind daher zunächst die N-Faktoren aller mit LST untersuchten stationären QSI-Moden abhängig von ihrer spannweitigen Wellenzahl $\beta$ und der Plattentiefenposition $x_{c} / c$ als farbliche Isokonturen dargestellt (exemplarisch für den Fall, in dem Heizreihe 1 mit einer Leistung von $P=14 \mathrm{~W}$ betrieben wird). In Isolinien sind die entsprechenden N-Faktoren für den ungeheizten Fall mit identischem Wertebereich dargestellt. Dabei ist zu erkennen, dass stationäre Moden allgemein durch die Heizung nur leicht destabilisiert sind. Die N-Faktor-Verläufe einiger wichtiger stationärer Moden sind als Liniendiagramm in Abbildung 7.3.10(b) dargestellt. Auch dabei werden nur geringe Änderungen festgestellt. Bei $x_{c} / c=0.35$, dem Ort der Messungen zur systematischen Variation der Heizleistung (Abschnitt 7.4), ergibt sich für Reihe 1 eine maximale Änderung der integralen Anfachung der Kontrollmode von $\Delta N=0.06$. Diese Abweichung entspricht weniger als $2 \%$ der integralen Anfachung zwischen der Aktuierungsposition $x_{c} / c=0.05$ und $x_{c} / c=0.35$. Für alle anderen Heizreihen ergeben sich sehr ähnliche Ergebnisse wie für Heizreihe 1.

Werden später signifikante Änderungen der Amplituden stationärer QSI in der Grenzschicht diskutiert, können diese nicht im Wesentlichen als Folge der linearen spannweitig mittleren Destabilisierung interpretiert werden. 
(a)

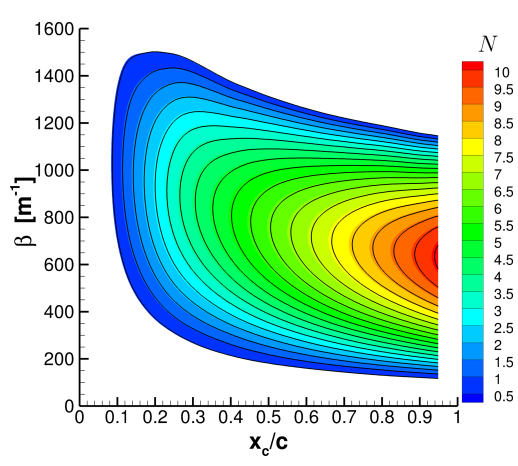

(b)

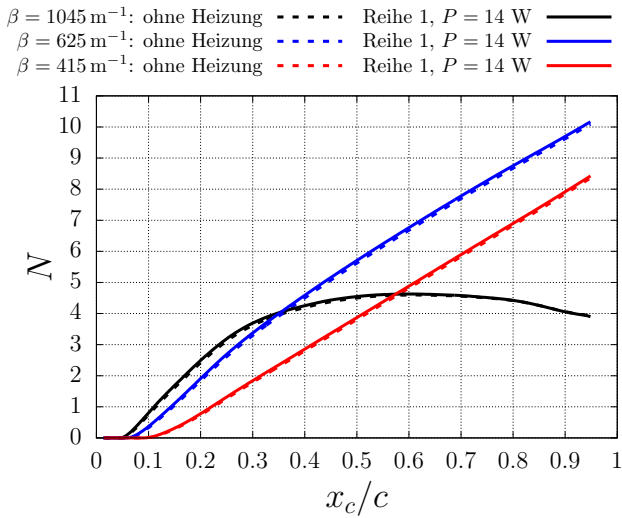

Abbildung 7.3.10: (a) Vergleich der N-Faktoren stationärer QSI in Abhängigkeit ihrer spannweitigen Wellenzahl $\beta$ und der Plattentiefenposition $x_{c} / c$ für den exemplarischen Fall, in dem Heizreihe 1 mit einer Leistung von $P=14 \mathrm{~W}$ betrieben wurde (Isokonturen) und den Fall ohne Heizung (Isolinien mit identischem Wertebereich) (b) Vergleich der N-Faktoren stationärer QSI für einzelne ausgewählte Moden

\section{Instationäre QSI}

Die Grenzschichtlösungen mit erhöhter Wandtemperatur wurden auch auf die Stabilität bzgl. instationärer QSI in einem breiten Frequenz- und Wellenzahlbereich untersucht. Für den exemplarischen Fall, in dem Heizreihe 1 mit $P=14 \mathrm{~W}$ betrieben wurde, sind in Abbildung 7.3.11(a) die an jeder Plattentiefenposition $x_{c} / c$ bzgl. $\beta$ maximalen N-Faktoren der untersuchten QSI-Moden in farblichen Isokonturen dargestellt. Zum Vergleich sind die bzgl. $\beta$ maximalen N-Faktoren der im ungeheizten Fall untersuchten QSI-Moden in Isolinien mit identischem Wertebereich dargestellt. Dabei ist ebenfalls zu erkennen, dass die im ungeheizten Fall angefachten Instabilitäten durch die Aktuierung nur geringfügig destabilisiert werden. Der Frequenzbereich angefachter instationärer Primärinstabilitäten wird im Plattentiefenbereich der Aktuierung (in diesem Beispielsfall bei $x_{c} / c=0.05$ ) und etwas stromab $\mathrm{zu}$ höheren Frequenzen erweitert. Etwa zwischen $x_{c} / c=0.05$ und $x_{c} / c=0.2$ gibt es einen Frequenzbereich mit schwacher integraler Anfachung, in dem für den ungeheizten Fall keine integrale Anfachung erwartet wird. 
(a)

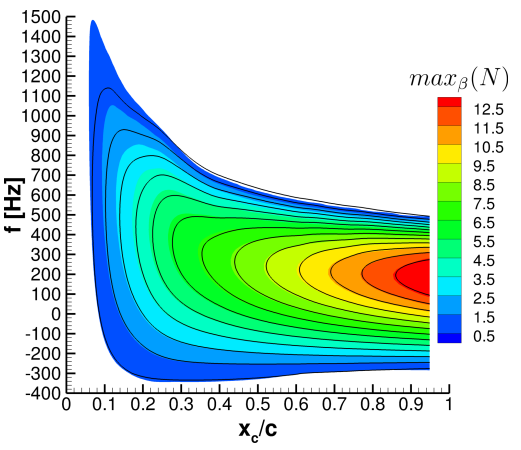

(b)

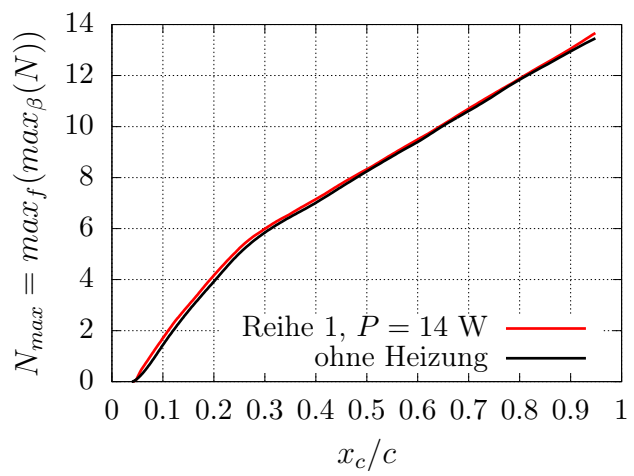

Abbildung 7.3.11: (a) Vergleich der bzgl. $\beta$ maximalen N-Faktoren untersuchter QSI-Moden für einen exemplarisch aktuierten Fall in farblichen Isokonturen und in Isolinien für den ungeheizten Fall (mit identischem Wertebereich) (b) Vergleich des bzgl. $f$ und $\beta$ maximalen N-Faktors $N_{\max }$

Um die zu erwartende geringe Destabilisierung laufender QSI durch die Heizung zu verdeutlichen, ist in Abbildung 7.3.11(b) für denselben exemplarischen Fall und den ungeheizten Fall der bzgl. $f$ und $\beta$ maximale N-Faktor $N_{\max }$ laufender QSI gegen $x_{c} / c$ aufgetragen. Dabei erkennt man, dass der Unterschied des N-Faktors zwischen dem aktuierten und nicht aktuierten Fall gegenüber dem Betrag der integralen Anfachung gering ist, dass also laufende Primärinstabilitäten durch den Einfluss der spannweitig mittleren Wandheizung nur geringfügig destabilisiert sind. An der Messposition $x_{c} / c=0.35$ zur systematischen Heizleistungsvariation (Abschnitt 7.4) ändert sich $N_{\max }$ gegenüber dem ungeheizten Fall im Mittel der unterschiedlichen Heizreihen um $\Delta N=0.12$. $N_{\max }$ erreicht an dieser Position bereits im Fall mit ausgeschaltetem Heizaktuator einen Wert von $N_{\max }=6.46$. Die zusätzliche integrale Anfachung durch die Anhebung der gemittelten Wandtemperatur beträgt also bei maximaler Heizleistung nur ca. $2 \%$ dieses Werts.

\subsection{Systematische Variation der Heizleistung}

In den vorangegangenen Abschnitten wurde die direkte Wirkung der Heizaktuierung auf die Grenzschichtströmung, auf ihre Stabilität und auf Hitzdrahtmessungen in der Grenzschicht diskutiert und für den größten Teil der Plattentiefe als gering bewertet. Es wurde ein linearer Zusammenhang 
zwischen der an einer Heizreihe angelegten Heizleistung und der Amplitude $\hat{T}_{W}$ der erzeugten spannweitigen Wandtemperaturperiodizität hergestellt. Im Folgenden soll die künstliche Anregung der ausgewählten stationären QSI-Kontrollmode anhand einer systematischen Variation der Heizleistung nachgewiesen und quantifiziert werden. Im Anschluss wird geprüft, welchen zusätzlichen Einfluss die systematisch variierte Aktuierung auf das stationäre und instationäre Störungsspektrum in der Grenzschicht hat. Die Hitzdrahtmessungen zur systematischen Variation der Heizleistung fanden als spannweitige Linienmessungen (siehe Abschnitt 5.1.4) in festem Wandabstand und an der festen Plattentiefenposition $x_{c} / c=0.35$ statt. Aus früheren Messungen im neu ausgelegten Prinzipexperiment [68] war bekannt, dass die künstlich angeregte stationäre Kontrollmode ihre maximale Amplitude etwa an dieser Plattentiefenposition erreicht.

Für diese Messungen wurde stets folgender Ablauf befolgt: Nach einer spannweitigen Linienmessung im ausgeschalteten Zustand des Heizaktuators wurde ein elektrischer Strom mit konstanter Leistung $P$ an einer der Heizreihen angelegt. Um zu einem stationären thermischen Gleichgewicht zu gelangen, wurde die folgende Linienmessung erst nach einer Wartedauer von ca. 15 Minuten bei konstanter Anströmung und Heizleistung durchgeführt. Mithilfe von Infrarot-Thermografie wurde zuvor sichergestellt, dass die Wandtemperaturverteilung sich nicht mehr signifikant änderte und ein stationärer Zustand erreicht wurde. Nach Abschluss dieser Linienmessung wurde die Leistung erhöht, die stationäre Wandtemperaturverteilung während derselben Wartedauer geprüft, eine weitere Linienmessung durchgeführt und der Vorgang wiederholt. So wurde die Heizleistung für jede Heizreihe zwischen $0 \mathrm{~W}$ und $14 \mathrm{~W}$ systematisch variiert und die deformierte Grenzschichtströmung bei $x_{c} / c=0.35$ untersucht. Ein solcher Ablauf wird im Folgenden als eine Messreihe bezeichnet.

\subsubsection{Künstliche Anregung der stationären Kontrollmode}

Die beschriebenen Linienmessungen wurden auf einer spannweitigen Strecke von $L=99.99 \mathrm{~mm}$ mit einer Schrittweite von $\Delta y_{c}=0.99 \mathrm{~mm}$ (siehe Abschnitt 5.1.4) durchgeführt. Für die Auswertung der Amplitude $\hat{u}_{s}(\beta)$ und Phase $\varphi(\beta)$ der stationären Kontrollmode mit $\beta=1047 \mathrm{~m}^{-1}$ bzw. $\lambda=6 \mathrm{~mm}$ wurde das spannweitige Spektrum der stationären $u_{s}$-Deformation auf einer spannweitig 
verkürzten Strecke von $16 \cdot \lambda=96 \mathrm{~mm}$ untersucht (für die Beschreibung der Auswertung stationärer Instabilitätsamplituden siehe Abschnitt 6.3.1).

Die wandnormale Positionierung der Hitzdrahtsonde unterliegt einer gewissen Ungenauigkeit, insbesondere zwischen unterschiedlichen Messreihen an unterschiedlichen Messtagen, da beim täglichen Kalibrieren der eingestellte Abstand der Sonde zur Modelloberfläche nicht in der nötigen Genauigkeit bewahrt werden konnte und nach der Kalibrierung neu eingestellt werden musste. Der Wandabstand wurde zunächst durch iterative Wiederholungsmessungen auf den Abstand des experimentellen Amplitudenmaximums der Kontrollmode eingestellt und betrug im Mittel $z=0.57(8) \mathrm{mm}$. Dieser Wert wurde im Nachhinein aus dem Vergleich des spannweitig gemittelten Geschwindigkeitsbetrags jeder Linienmessung mit dem experimentell bestimmten spannweitig gemittelten Grenzschichtprofil bestimmt. Der angegebene Fehler entspricht der Streuung der so bestimmten Wandabstände über alle Linienmessungen der systematischen Variation aufgrund der geschilderten Einschränkungen. Wie in Abschnitt 7.2 diskutiert wurde, wird auch im Fall des ausgeschalteten Heizaktuators bereits eine gewisse Amplitude der spannweitigen $u_{s^{-}}$ Deformation mit $\lambda=6 \mathrm{~mm}$ beobachtet, welche wegen der eingeschränkten Reproduzierbarkeit des Wandabstandes in unterschiedlichen Messreihen leicht verschiedene Werte aufweist. Innerhalb einer Messreihe, in der jeweils die Leistung einer einzelnen Heizreihe systematisch variiert und untersucht wurde, wurde der wandnormale Abstand nominell nicht verändert.

Die aus dem spannweitigen Spektrum der $u_{s}$-Deformation ausgewerteten Amplituden $\hat{u}_{s}$ der angeregten Kontrollmode sind in Abbildung 7.4.1 für alle Heizreihen abhängig von der Heizleistung $P$ dargestellt, wobei die Amplitude im ausgeschalteten Zustand des Aktuators $\hat{u}_{s, 0}$ jeweils abgezogen wurde. Der Wert der abgezogenen Amplitude betrug ca. 2\% der Grenzschichtrandgeschwindigkeit $q_{e}$. Anstatt die erzeugte Amplitude der angeregten stationären Kontrollmode weiter abhängig von der Heizleistung zu diskutieren, soll sie im Folgenden abhängig von der Amplitude der erzeugten spannweitigen Wandtemperaturperiodizität $\hat{T}_{W}$ diskutiert werden, da diese für den physikalischen Rezeptivitätsmechanismus relevanter ist als die Heizleistung. Die für eine bestimmte Störungsanregung nötige Heizleistung ist stark von der gewählten technischen Implementierung abhängig und somit kaum auf andere Fälle übertragbar. In Abschnitt 7.3.2 wurde für alle Heizreihen jeweils ein linearer Zusammenhang zwischen der Amplitude $\hat{T}_{W}$ und der Heizleistung $P$ hergestellt. 


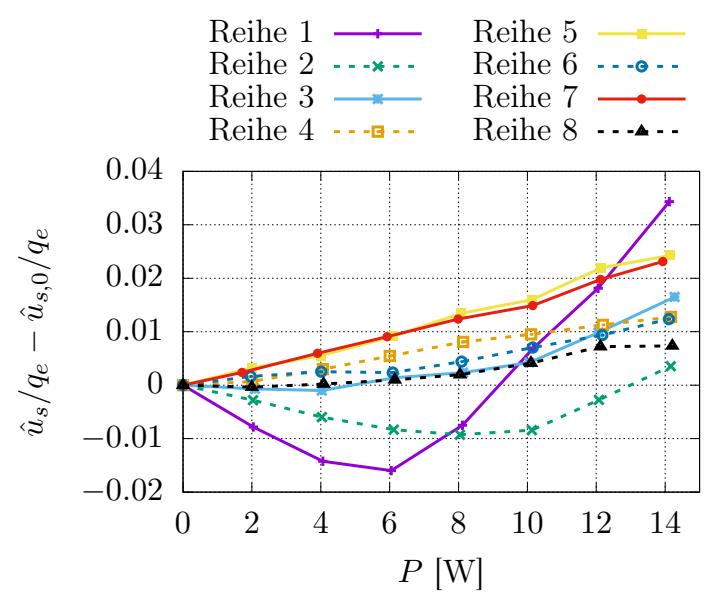

Abbildung 7.4.1: Amplitude der aktuierten stationären QSI-Mode in konstantem Wandabstand bei einer Plattentiefe von $x_{c} / c=0.35$ in Abhängigkeit der Heizleistung für die verschiedenen Heizreihen

Die bereits in Abbildung 7.4.1 dargestellten Instabilitätsamplituden sind in Abbildung 7.4.2(a) abhängig von $\hat{T}_{W}$ dargestellt. Der Wert der Amplitude bei ausgeschaltetem Aktuator wurde dabei jeweils abgezogen. In Abbildung 7.4.2(b) ist die zugehörige Phasenlage $\varphi$ als Vielfaches von $\pi$ dargestellt, wobei die Phasenlage $\varphi_{0}$ bei ausgeschaltetem Aktuator abgezogen wurde. Man erkennt, dass beim Einsatz der Heizreihen 1 und 2 die Amplitude mit steigendem Wert von $\hat{T}_{W}$ zunächst abnimmt und erst nach Überwindung eines Minimums wieder zunimmt. Bei stärkerer Aktuierung zeigen alle Heizreihen einen nahezu linearen Zusammenhang zwischen $\hat{T}_{W}$ und der Amplitude der künstlich angeregten Kontrollmode. In Abbildung 7.4.2(b) ist zu erkennen, dass bei den ersten beiden Heizreihen die Aktuierung mit maximaler Heizleistung zu einer betragsmäßig besonders großen Phasenverschiebung führt. Bei Heizreihe 1 wird eine Verschiebung um $-\pi$ erreicht. Dies entspricht einer spannweitigen Verschiebung der Wirbelachsen um $\lambda / 2$ in $+y_{c}$-Richtung. In Abschnitt 7.3.2 wurde darauf hingewiesen, dass die Phasenlage der erzeugten Wandtemperaturperiodizität selbst im Wesentlichen unabhängig von der Heizleistung ist, sodass die beobachtete Phasenverschiebung einen anderen Grund haben muss.

Das Phänomen einer mit zunehmender Anregungsstärke zunächst abnehmenden Amplitude der eigentlich angeregten stationären QSI-Mode beobachteten Lohse et al. [69] und Berthold [7] ebenfalls. Lohse et al. [69] beobachteten das 
(a)

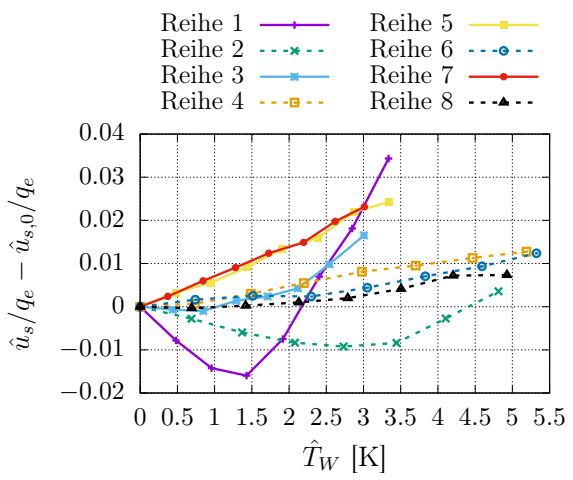

(b)

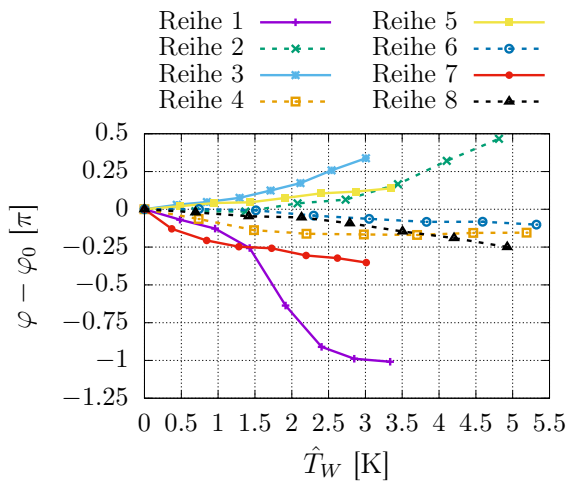

Abbildung 7.4.2: (a) Amplitude der aktuierten stationären QSI-Mode $(\beta=$ $1047 \mathrm{~m}^{-1}$ ) in konstantem Wandabstand bei einer Plattentiefe von $x_{c} / c=0.35$ in Abhängigkeit der jeweils erzeugten Wandtemperaturperiodizität für die verschiedenen Heizreihen (b) Phasenlage der aktuierten stationären QSI-Mode aus denselben Messungen

Phänomen bei pneumatischer Anregung, gaben dafür aber keinen Erklärungsansatz an. Berthold [7] beobachtete das Phänomen sowohl für Heizanregung als auch für pneumatische Anregung. Im Falle der Heizanregung gab auch er keinen Erklärungsansatz an. Im Falle der pneumatischen Anregung wurde eine spannweitig periodische Anordnung von Schlitzen verwendet. Die Anregung stationärer QSI bei ausgeschaltetem Aktuator wurde mit einer Oszillation der Luft im Schlitz ,ähnlich zu einem Helmholtz-Resonator“ [7] in Verbindung gebracht, welche durch das pneumatische Ausblasen mit zunehmendem Volumenstrom unterbunden werde. Dadurch nehme zunächst die QSI-Amplitude ab, bevor das pneumatische Ausblasen selbst zu einer signifikanten Anregung stationärer QSI führe. Diese Erklärung erscheint nicht plausibel, da es sich bei einer Oszillation im Schlitz wie bei einem HelmholtzResonator um ein instationäres Phänomen handelt, von dem keine Anregung einer stationären QSI-Mode zu erwarten ist.

Im Folgenden soll daher ein eigener Erklärungsansatz für die bei geringen Werten von $\hat{T}_{W}$ zunächst reduzierte Amplitude der Kontrollmode vorgestellt werden. Die Amplitude und Phasenlage der Kontrollmode an der Messposition $x_{c} / c=0.35$ wird ohne Heizaktuierung durch die Überlagerung stromauf liegender Anteile des spannweitigen Rauigkeitsspektrums mit der Wellenlänge $\lambda=6 \mathrm{~mm}$ bestimmt. Auch ohne aktive Aktuierung ist die Kontrollmode 
dadurch bereits mit endlicher Amplitude angeregt. Bei Betrieb einer der Heizreihen besitzt die zusätzlich künstlich eingebrachte Störung einen zunächst unbekannten und vom Rezeptivitätsprozess der Heizanregung abhängigen Phasenbezug zu dieser Überlagerung. Eine abnehmende Amplitude der Kontrollmode an der Messposition für bestimmte Werte von $\hat{T}_{W}$ weist darauf hin, dass dieser Phasenbezug teilweise destruktiv ist. Die Summe der Anregungen führt in einem Bereich der Anregungsstärke zu einer verringerten Amplitude. Man bezeichne den Anteil der Grenzschichtdeformation mit $\lambda=6 \mathrm{~mm}$ allein durch Rauigkeiten als $u_{s, 0}^{*}\left(y_{c}\right)$ und den Anteil durch die Heizaktuierung als $u_{s, 1}^{*}\left(y_{c}\right)$. Beide Anteile lassen sich wie folgt als harmonische Funktion der Spannweite $y_{c}$ modellieren:

$$
\begin{aligned}
& u_{s, 0}^{*}\left(y_{c}\right)=\hat{u}_{s, 0} \sin \left(\frac{2 \pi}{\lambda} y_{c}\right) \\
& u_{s, 1}^{*}\left(y_{c}\right)=\hat{u}_{s, 1} \sin \left(\frac{2 \pi}{\lambda} y_{c}-\varphi_{1}\right)
\end{aligned}
$$

Dabei wurde die Phasenlage des Rauigkeitsanteils zu $\varphi_{0}=0$ definiert, da $\varphi_{1}$ relativ zu dieser Phasenlage und damit als Phasenrelation der beiden Deformationsanteile betrachtet werden soll. Die Summe der beiden Deformationen ergibt einen deformierten Geschwindigkeitsverlauf $u_{s, g e s}$ :

$$
u_{s, g e s}\left(y_{c}\right)=u_{s, 0}^{*}\left(y_{c}\right)+u_{s, 1}^{*}\left(y_{c}\right)
$$

dessen reellwertiger harmonischer Anteil bei $\lambda=6 \mathrm{~mm}$ als $u_{s}^{*}\left(y_{c}\right)$ bezeichnet wird. Die Amplitude dieses Anteils wird nun auch als $\hat{u}_{s}$ bezeichnet.

Wie oben beschrieben, hat $\hat{u}_{s, 0}$ etwa einen Wert von $\hat{u}_{s, 0}=0.02 q_{e}$. Die resultierende Amplitude $\hat{u}_{s}$ hängt wie in Abbildung 7.4.3 gezeigt je nach Phasenrelation $\varphi_{1}$ verschieden von $\hat{u}_{s, 1}$ ab. Für Phasenrelationen im Bereich $0 \leq \varphi_{1} \leq \pi / 2$ erwartet man einen monotonen Anstieg der resultierenden Kontrollmodenamplitude. Für den Wertebereich $\pi / 2<\varphi_{1} \leq \pi$ erwartet man, dass die Amplitude $\hat{u}_{s}$ für bestimmte Werte von $\hat{u}_{s, 1}$ reduziert ist. Selbes gilt für den Wertebereich $\pi \leq \varphi_{1}<3 \pi / 2$. Für den Wertebereich $3 \pi / 2 \leq \varphi_{1} \leq 2 \pi$ erwartet man wieder einen rein monotonen Anstieg. Im Folgenden soll in den gemessenen $u_{s}\left(y_{c}\right)$ Verläufen der Teil der Grenzschichtdeformation, der allein auf die künstliche Anregung durch die Heizung zurückzuführen ist, von dem Teil als Folge von Rauigkeit getrennt werden. Dazu wurde der spannweitige $u_{s}$-Verlauf im ausgeschalteten Fall $u_{s, 0}\left(y_{c}\right)$ von dem jeweiligen Verlauf im selben spannweitigen Bereich und bei gleichem Wandabstand im jeweils aktuierten Fall $u_{s, a c t}\left(y_{c}\right)$ subtrahiert. Diese Differenz wird mit

$$
a_{s}\left(y_{c}\right):=u_{s, a c t}\left(y_{c}\right)-u_{s, 0}\left(y_{c}\right)
$$




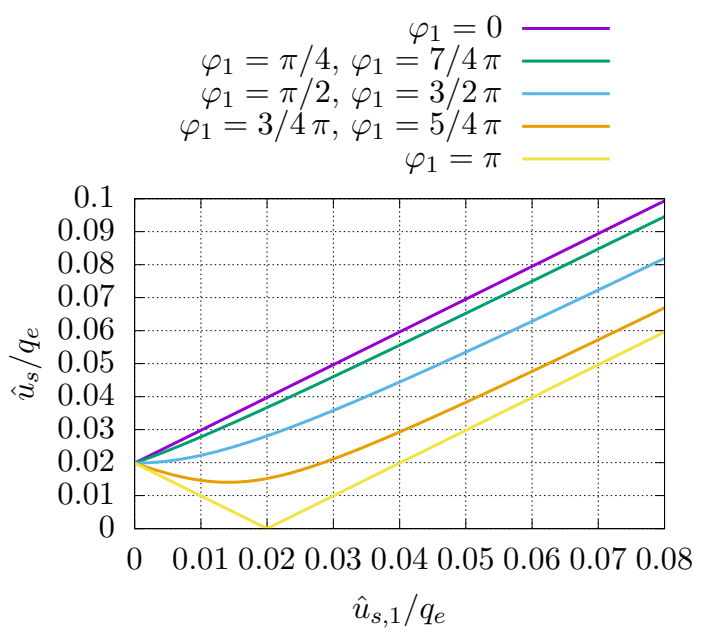

Abbildung 7.4.3: Modellierte Amplitude der Kontrollmode aus der Summe der Teildeformationen aus Rauigkeiten und Heizaktuierung abhängig von der Amplitude der Teildeformation aus Heizaktuierung $\hat{u}_{s, 1}$ für verschiedene Werte der Phasenrelation $\varphi_{1}$

bezeichnet. Wie gut die gewünschte Isolierung der Aktuierungswirkung erreicht wird, hängt einerseits von der Reproduzierbarkeit der Linienmessungen ab. Zusätzlich hängt es aber auch davon ab, ob die Aktuierungswirkung im Wesentlichen additiv der Rauigkeitswirkung überlagert ist, wie im geschilderten Modell anhand von Gleichung 7.4.3 angenommen.

Um diese Trennung exemplarisch für eine Heizreihe und eine Heizleistung zu demonstrieren, sind in Abb. 7.4.4 der gemessene spannweitige $u_{s}$-Verlauf im Fall ohne Aktuierung $u_{s, 0}$, derjenige im Fall mit Betrieb der Reihe 1 und $P=14 \mathrm{~W} u_{s, a c t}$ und der resultierende Verlauf von $a_{s}$ dargestellt. Im Fall ohne Aktuierung ist $\mathrm{zu}$ erkennen, dass $u_{s, 0}$ hauptsächlich langwellig deformiert ist. Die mit Heizreihe 1 eingebrachte zusätzliche Deformation ist in $u_{s, a c t}$ zwar erkennbar, aber die Überlagerung des spektralen Anteils der langwelligen stationären QSI und der künstlich angeregten Kontrollmode macht die Periodizität mit $\lambda=6 \mathrm{~mm}$ nicht offensichtlich. Im spannweitigen Verlauf der isolierten Aktuierungswirkung $a_{s}$ ist dieser Anteil offensichtlich und besitzt eine große Amplitude. Das räumliche Spektrum des spannweitigen Verlaufs $a_{s}\left(y_{c}\right)$ enthält also die Amplituden und Phasenlagen der durch die Aktuierung angeregten stationären QSI-Mode, unabhängig von dem durch Rauigkeit angeregten Anteil, der in $u_{s, 0}$ enthalten ist. Diese Amplituden 


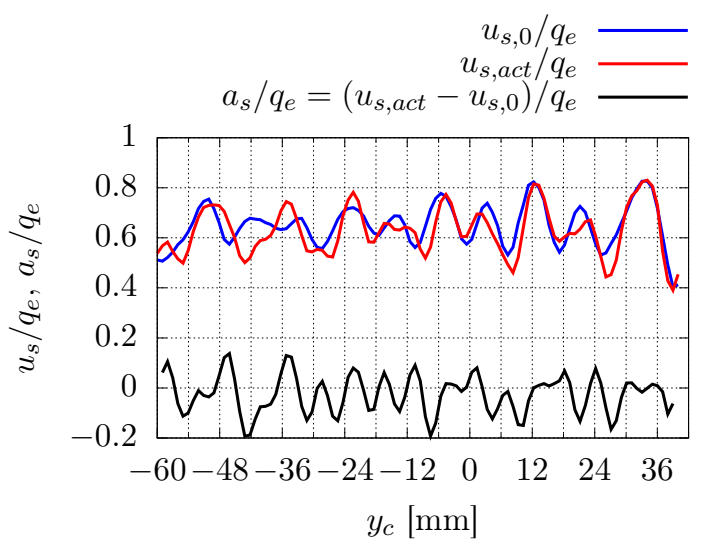

Abbildung 7.4.4: Exemplarische isolierte Aktuierungswirkung $a_{s} / q_{e}$, gebildet aus dem spannweitig gemessenen $u_{s}$-Verlauf bei Betrieb von Reihe 1 mit $P=14 \mathrm{~W}$ und demjenigen im Fall ohne Aktuierung (Messposition $x_{c} / c=0.35$ )

(a)
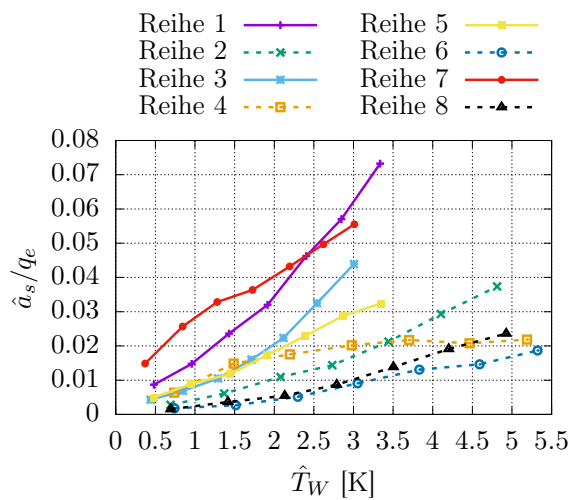

(b)

Reihe $1 \longrightarrow$ Reihe 5

Reihe $2 \ldots *$-.. Reihe $6 \ldots-\cdots$

Reihe $3 \multimap \quad$ Reihe $7 \longrightarrow$

Reihe 4 ... - Reihe 8 .......

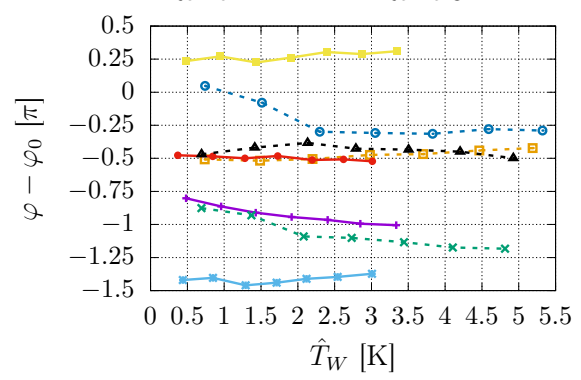

Abbildung 7.4.5: (a) Amplituden $\hat{a}_{s}$ mit der aktuierten Wellenzahl $\beta=1047 \mathrm{~m}^{-1}$ in Abhängigkeit von der Amplitude der Wandtemperaturperiodizität für alle untersuchten Heizreihen (b) Die dazugehörigen Phasenlagen mit abgezogener Phasenlage der nicht aktuierten Referenzmessung $\phi_{0}$

$\hat{a}_{s}(\beta)$ und zugehörigen Phasenlagen $\varphi(\beta)$ werden mithilfe einer FourierTransformation ausgewertet, wie in Abschnitt 6.3.1 für $\hat{u}_{s}$ beschrieben. Sie sind für alle Heizreihen in den Abbildungen 7.4.5(a) und 7.4.5(b) dargestellt. 
Die Amplitudenverläufe in Abbildung 7.4.5(a) zeigen bereits bei der kleinsten untersuchten Heizleistung für alle Heizreihen eine Amplitude $\hat{a}_{s}>0$, was bei erfolgreicher Trennung der beiden Anteile zur Grenzschichtdeformation aus den beschriebenen Ursachen zu erwarten ist. Für alle Heizreihen außer Reihe 4 zeigt sich zudem ein monoton mit $\hat{T}_{W}$ steigender Amplitudenverlauf. Beim Vergleich mit Abbildung 7.4.2(a) erkennt man, dass die maximal erreichte Amplitude $\hat{a}_{s}$ für Heizreihe 1 etwa doppelt so groß ist wie die maximal erreichte Amplitude $\hat{u}_{s}$. Ohne destruktive Überlagerung wäre also mit Reihe 1 eine viel größere Amplitude der Kontrollmode erzeugt worden. Die bei hohen Werten von $\hat{T}_{W}$ erreichte Steigung der Amplitude $\hat{a}_{s}$ bzgl. $\hat{T}_{W}$ unterscheidet sich für die verschiedenen Heizreihen stark. Die unterschiedlichen Heizreihen sind also verschieden effektiv bei der Anregung der Kontrollmode.

In Abbildung 7.4.5(b) sind die Verläufe der Phasenlage der $a_{s}$-Periodizität mit der aktuierten Wellenlänge abhängig von $\hat{T}_{W}$ für alle Heizreihen dargestellt. Dabei wurde die Phasenlage der $u_{s}$-Periodizität (mit $\lambda=6 \mathrm{~mm}$ ) im nicht aktuierten Referenzfall jeweils abgezogen, wodurch die dargestellte Phasenlage als Phasenrelation zur Anregungswirkung durch Rauigkeit interpretiert werden kann. Es ist zu beachten, dass die Auswertung der Phasenlage natürlich invariant bzgl. Addition oder Subtraktion von $2 \pi$ bzw. einer spannweitigen Verschiebung von $\lambda$ ist. Für alle Heizreihen ist zu beobachten, dass die Phasenlage der $a_{s}$-Periodizität sich bei Steigerung von $\hat{T}_{W}$ nur geringfügig ändert. Die jeweilige gemittelte Phasenrelation zur Periodizität im ausgeschalteten Fall, also zur reinen Summe der Rauigkeitsanregungen, unterscheidet sich für die verschiedenen Heizreihen aber deutlich. Der Wert dieser Phasenrelation wird durch die spannweitige Lage der Heizelemente relativ zu den Wirbelachsen der etablierten QSI-Kontrollmode bestimmt. Damit ist der jeweilige Wert hauptsächlich geometrisch bedingt. Die Beobachtung der jeweils konstanten aber untereinander verschiedenen Phasenrelationen für verschiedene Heizreihen ist ein weiterer Hinweis darauf, dass die beschriebene Trennung der beiden Anregungswirkungen erfolgreich war. Die Anregungen mit den Heizreihen 1 und 2 weisen am stärksten einen destruktiven Phasenbezug zur Rauigkeitsanregung auf. Es waren auch diese beiden Heizreihen, für die in Abbildung 7.4.2(a) eine Reduzierung der Kontrollmoden-Amplitude beobachtet wurde. Dies unterstützt die Interpretation der abnehmenden Amplitude als Resultat einer destruktiven Phasenbeziehung zwischen der Heizanregung und der Anregung durch Rauigkeit.

Zur Überprüfung dieser Interpretation wird exemplarisch für Heizreihe 1 in der oben beschriebenen Modellierung (Gleichungen 7.4.1, 7.4.2 und 7.4.3) 


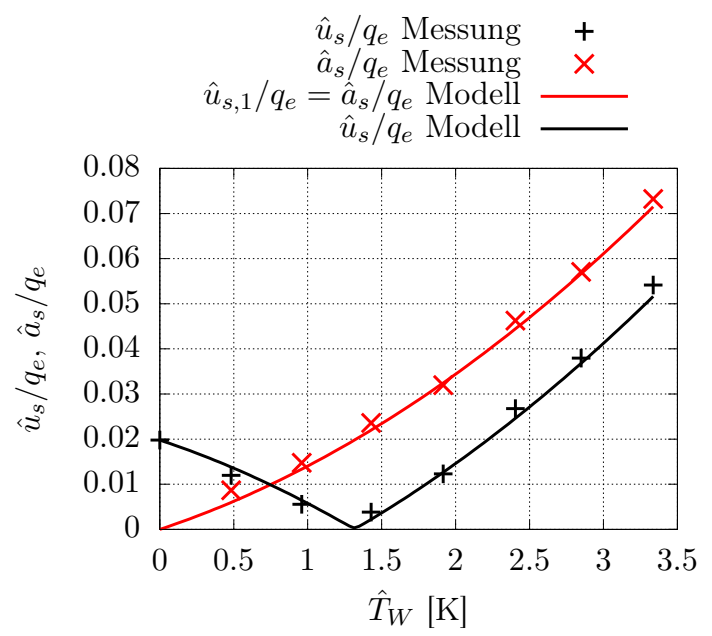

Abbildung 7.4.6: Modellierte resultierende Amplitude der Kontrollmode für Heizreihe $1 \mathrm{im}$ Vergleich zur reell gemessenen Amplitude $\hat{u}_{s}$

die Phasenrelation $\varphi_{1}$ konstant zu dem Wert gewählt, der bei hohen Werten von $\hat{T}_{W}$ als Phasenrelation der $a_{s}$-Periodizität zur Rauigkeitsanregung ausgewertet wurde, also nahezu $-\pi$. Es wird $\hat{u}_{s, 1}=\hat{a}_{s}$ gesetzt und $\hat{a}_{s}\left(\hat{T}_{W}\right)$ als quadratrische Funktion modelliert. Als Amplitude der Rauigkeitswirkung $\hat{u}_{s, 0}$ wird der experimentell bestimmte Wert gewählt. Berechnet man wie oben die Summe $u_{s, g e s}\left(y_{c}\right)$ aus den modellierten Teildeformationen und bestimmt die Amplitude $\hat{u}_{s}$ des harmonischen Anteils bei $\lambda=6 \mathrm{~mm}$, erhält man den Verlauf in Abbildung 7.4.6. Zum Vergleich ist die Amplitude der reell gemessenen $u_{s}$-Deformation mit $\lambda=6 \mathrm{~mm}$ ebenfalls eingezeichnet. Die gemessenen Werte der Amplitude lassen sich durch den modellierten Verlauf mit geringen Abweichungen gut nachvollziehen. Dies bestätigt den Erklärungsansatz, auf dem die Modellierung basiert. Die Tatsache, dass die Heizanregung mit Reihe 1 einen nahezu rein destruktiven Phasenbezug zur Rauigkeitsanregung hat, war bei der geometrischen Auslegung der Heizelemente nicht beabsichtigt, zeigt aber eine mögliche Methode zur Transitionskontrolle auf. Ist die spannweitige Wellenlänge und Phasenlage der natürlich dominanten QSI-Mode bspw. durch geeignete Sensorik bekannt, könnte ein Aktuator eine zusätzliche Anregung mit destruktivem Phasenbezug dazu erzeugen und so die natürliche Transition verzögern. Der Erklärungsansatz ist auch in der Lage, dasselbe Phänomen für die in [69] und [7] untersuchten Aktuatoren zu erklären, da dort ebenfalls signifikante Rauigkeiten mit der spannweitigen Wellenlänge der anzuregenden Mode vorhanden waren. 
(a)

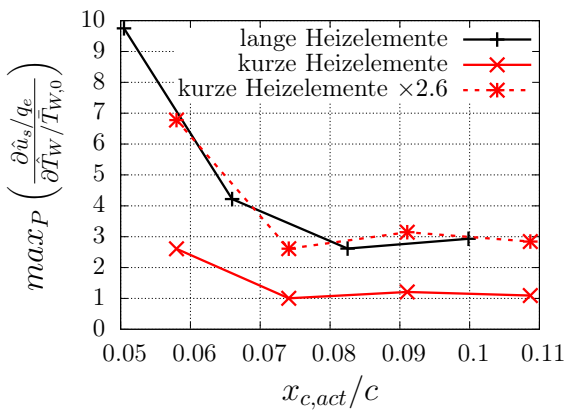

(b)

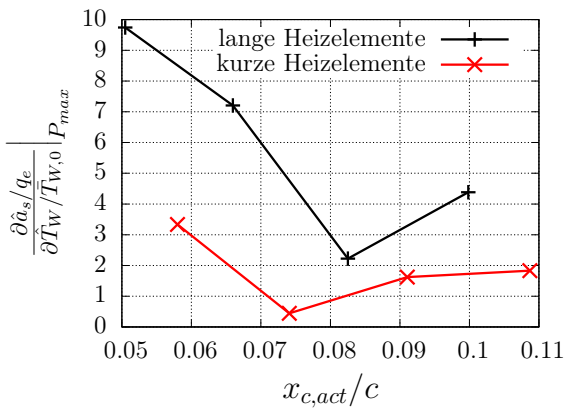

Abbildung 7.4.7: (a) Effizienz der Anhebung der Kontrollmodenamplitude $\hat{u}_{s}$ bzgl. der Wandtemperaturperiodizität $\hat{T}_{W}$ abhängig vom Ort der Anregung $x_{c, a c t}$ (b) Analoge Effizienz basierend auf der isolierten Aktuierungswirkung $\hat{a}_{s}$

In den Amplitudenverläufen $\hat{u}_{s}\left(\hat{T}_{W}\right)$ in Abbildung 7.4.2(a) wurden für die unterschiedlichen Heizreihen unterschiedliche maximale Steigungen der QSIAmplitude abhängig von der verwendeten Anregungsamplitude $\hat{T}_{W}$ beobachtet. Das bedeutet, dass für eine Steigerung der Kontrollmoden-Amplitude $\hat{u}_{s}$ an der Messposition eine unterschiedlich starke Erhöhung der Anregungsamplitude $\hat{T}_{W}$ (bzw. der Heizleistung $P$ ) an der Aktuatorposition nötig ist. Daher sollen diese Steigungen im Folgenden als eine Effizienz der Störungseinbringung bis zur Messposition interpretiert werden. Für die Darstellung dieser Effizienz wurde die Amplitude der Kontrollmode $\hat{u}_{s}$ durch die Grenzschichtrandgeschwindigkeit $q_{e}$ an der Messposition und die Amplitude der Wandtemperaturperiodizität $\hat{T}_{W}$ durch die jeweilige spannweitig gemittelte Wandtemperatur an der Heizreihenposition im nicht aktuierten Fall $\bar{T}_{W, 0}$ entdimensionalisiert. In Abbildung 7.4.7(a) sind die resultierenden Effizienzwerte der Störungseinbringung abhängig von der jeweiligen mittleren Heizreihenposition aufgetragen. Dabei wurde zwischen langen und kurzen Heizelementen unterschieden, also den Reihen 1,3,5 und 7 bzw. Reihen 2,4,6 und 8. Es ist zu erkennen, dass die Effizienz mit steigender Plattentiefenposition des Anregungsorts im Wesentlichen abnimmt. Dies entspricht dem erwarteten Verhalten, da Instabilitäten in der Nähe ihrer Staulinie üblicherweise am effizientesten angeregt werden können, siehe Abschnitt 2.3. Des Weiteren ist die Effizienz bei vergleichbarer Anregungsposition für lange Heizelemente stets größer als für kurze Heizelemente. Dies legt die Interpretation nahe, dass die Anregung einer stationären QSI-Mode durch Heizaktuierung in signifikantem Maß entlang einer gewissen Strecke wirksam ist. Die Länge der Heizelemente 
unterscheidet sich um den Faktor 2, die dargestellte Effizienz im Mittel sogar um einen größeren Faktor $(\approx 2.6)$, wie in der Abbildung als gestrichelte Linie angedeutet ist. Diese Beobachtung gilt auch für die Effizienz, die man auf Grundlage der isolierten angeregten Amplitude $\hat{a}_{s}$ auswertet, welche in Abbildung 7.4.7(b) analog zu Abb. 7.4.7(a) gezeigt wird. Dabei wurde allerdings nicht der maximale Gradient, sondern derjenige bei maximaler Heizleistung ausgewertet. Möchte man mit einem technischen Heizaktuator eine Kontrollmode anregen, muss neben dieser unterschiedlichen Effizienz der Leistungsbedarf beachtet werden. Im Abschnitt 7.3.2 wurde beschrieben, dass mit langen Heizelementen zur Erzeugung derselben Amplitude $\hat{T}_{W}$ im Mittel eine um $60 \%$ höhere Heizleistung nötig ist als mit kurzen Heizelementen. Zumindest in der hier untersuchten technischen Realisierung reduziert dies den technischen Vorteil von langen Heizelementen.

Es wurde also gezeigt, dass die Kontrollmode durch spannweitig periodische Wandheizung erfolgreich angeregt werden konnte. Dabei wurde eine destruktive Überlagerung mit der bereits durch Rauigkeiten angeregten Mode selber spannweitiger Wellenlänge beobachtet. Es konnte gezeigt werden, dass die Amplitude der angeregten Kontrollmode durch Variation der Heizleistung bzw. $\hat{T}_{W}$ gesteuert werden kann. Die Effizienz der Störungseinbringung ist für eine Anregung nahe am Neutralpunkt der Kontrollmode größer als stromab und für die langen Heizelemente größer als für die kurzen.

\subsubsection{Nichtlineare Wechselwirkungen stationärer Störungen}

Neben der beabsichtigten Anregung der stationären Kontrollmode mit der Wellenlänge $\lambda=6 \mathrm{~mm}$ bzw. der Wellenzahl $\beta=1047 \mathrm{~m}^{-1}$ sind im spannweitigen Spektrum der stationären Grenzschichtdeformation im Rahmen der Heizleistungsvariation auch andere Änderungen zu beobachten. Exemplarisch sind in Abbildung 7.4.8 die spannweitigen Spektren der jeweils gemessenen stationären $u_{s}$-Deformation in der Grenzschicht bei $x_{c} / c=0.35$ für Fälle stromab von Heizreihe 1 bei unterschiedlicher Temperaturamplitude $\hat{T}_{W}$ dargestellt. Da hier nun nicht hauptsächlich die Amplitude der Kontrollmode betrachtet, sondern ein Überblick über das stationäre Störspektrum diskutiert wird, wurde zur Berechnung des Spektrums $\hat{u}_{s}(\beta)$ die ausgewertete spannweitige Strecke nicht wie bisher in Abschnitt 7.4 auf ein Vielfaches der Kontrollmoden-Wellenlänge reduziert. Nun ist eine spektrale Auflösung von $\Delta \beta=62.2 \mathrm{~m}^{-1} \mathrm{zu}$ beachten. Die hier beobachtete Anhebung der Amplitude 


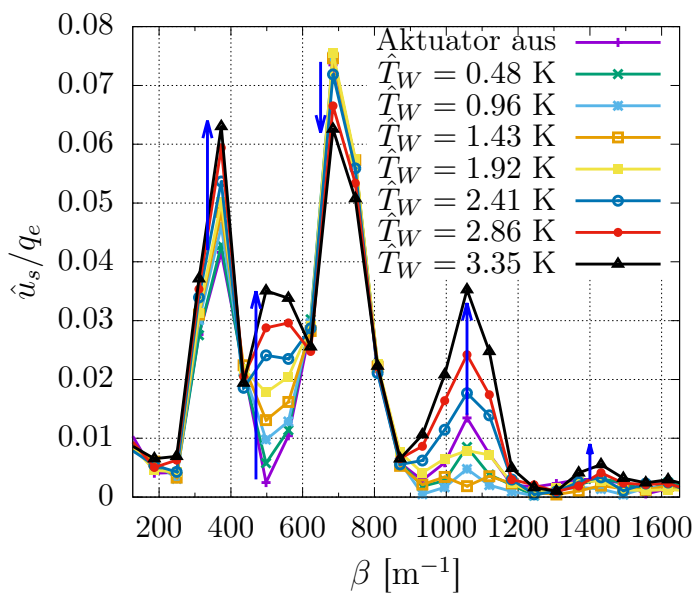

Abbildung 7.4.8: Spannweitiges Spektrum der bei konstantem Wandabstand gemessenen stationären $u_{s}$-Deformation bei Betrieb der Heizreihe 1 mit unterschiedlichen Heizleistungen

bei $\beta=1058 \mathrm{~m}^{-1}$ wird im Folgenden als die Amplitude einer spannweitigen Periodizität mit $\beta=1047 \mathrm{~m}^{-1}$ interpretiert, da diese Periodizität geometrisch durch den Aktuatorentwurf vorgegeben ist. Bei der Diskussion anderer spektraler Anteile als der Kontrollmode muss beachtet werden, dass die zugrundeliegenden Linienmessungen im Wandabstand des Amplitudenmaximums der Kontrollmode durchgeführt wurden. Dieser Wandabstand entspricht nicht exakt dem Wandabstand des Amplitudenmaximums anderer Moden, weshalb die quantitativen Amplitudenwerte nur eingeschränkt aussagekräftig sind.

Im Diagramm ist für kleine Werte von $\hat{T}_{W}$ die schon in Abschnitt 7.4.1 diskutierte anfängliche Reduktion der Kontrollmodenamplitude zu sehen. Für $\hat{T}_{W}>1.43 \mathrm{~K}$ steigt die Amplitude der Kontrollmode aber monoton mit $\hat{T}_{W}$. Des Weiteren wird eine Anhebung der Amplitude der stationären $u_{s^{-}}$ Deformation bei $\beta=498 \mathrm{~m}^{-1}$ beobachtet. Diese Wellenzahl entspricht im Rahmen der spektralen Auflösung nahezu $\beta=0.5 \cdot 1047 \mathrm{~m}^{-1}=523.5 \mathrm{~m}^{-1}$, der Hälfte der Kontrollmodenwellenzahl. Die Amplitude bei dieser Wellenzahl wird mit wachsendem $\hat{T}_{W}$ monoton angehoben. Dies weist darauf hin, dass die Aktuierung spannweitige Inhomogenitäten enthält, die zu einer zusätzlichen Anregung bei der doppelten Wellenlänge bzw. der halben Wellenzahl führen. Weitere lokale Maxima werden im spannweitigen Spektrum schon ohne Aktuierung bei $\beta=373 \mathrm{~m}^{-1}$ und $\beta=684 \mathrm{~m}^{-1}$ beobachtet. Ähnliche spektrale 
Anteile wurden in Abschnitt 7.2 diskutiert, wobei dort aufgrund der geringeren Länge des spannweitigen Messbereichs eine gröbere spektrale Auflösung relevant war. Das lokale Maximum bei $\beta=373 \mathrm{~m}^{-1}$ wird als Konsequenz der Welligkeit der Heizaktuatoroberfläche interpretiert. Die Amplitude dieses lokalen Maximums wird mit steigendem $\hat{T}_{W}$ deutlich angehoben. Das lokale Maximum bei $\beta=684 \mathrm{~m}^{-1}$ liegt in dem Wellenzahlbereich der gemäß LST besonders integral angefachten stationären QSI (siehe Abschnitt 6.2). Die Amplitude bei dieser Wellenzahl ändert sich bei Variation der Heizleistung kaum, bis sie bei hohen Werten von $\hat{T}_{W}$ etwas abnimmt. Bei hohen Werten von $\hat{T}_{W}$ wird zusätzlich eine leichte Anhebung der Amplitude bei der Wellenzahl $\beta=1431 \mathrm{~m}^{-1}$ beobachtet.

In Abschnitt 2.2 wurde beschrieben, dass in der linearen Stabilitätstheorie nichtlineare Terme der Stördifferentialgleichungen vernachlässigt werden. Sind solche Terme aufgrund großer Störungsamplituden nicht mehr vernachlässigbar, können aus Termen mit Produkten der Störungsgrößen nichtlineare Wechselwirkungen resultieren. Dabei hat die Wechselwirkung zweier QSIModen eine Wirkung auf eine dritte, deren Wellenzahl der Summe oder Differenz der einzelnen Wellenzahlen entspricht. Diese Eigenschaft geht aus dem Exponentialansatz (Gleichung 2.2.7) hervor, da im Produkt zweier Ansätze die Phasenfunktionen addiert werden. Auffällig ist bei den Änderungen der lokalen Maxima in Diagramm 7.4.8 also, dass die Wellenzahldifferenz $\Delta \beta=1058 \mathrm{~m}^{-1}-684 \mathrm{~m}^{-1}$ (bis auf Rundungsfehler) der Wellenzahl des lokalen Maximums bei $\beta=373 \mathrm{~m}^{-1}$ entspricht. Dies weist auf eine nichtlineare Wechselwirkung der Kontrollmode und der stationären QSI mit $\beta=684 \mathrm{~m}^{-1}$ hin, welche eine Anhebung der stationären QSI bei $\beta=373 \mathrm{~m}^{-1}$ zur Folge hat. Eine nichtlineare Wechselwirkung der Kontrollmode und der Mode bei $\beta=373 \mathrm{~m}^{-1}$ kann die beschriebene Reduzierung der Amplitude bei $\beta=1058 \mathrm{~m}^{-1}-373 \mathrm{~m}^{-1} \approx 684 \mathrm{~m}^{-1}$ erklären, ebenso wie die Anhebung der Amplitude bei $\beta=1058 \mathrm{~m}^{-1}+373 \mathrm{~m}^{-1} \approx 1431 \mathrm{~m}^{-1}$.

Alle beschriebenen Phänomene, die mit nichtlinearen Wechselwirkungen in Verbindung gebracht wurden, werden erst bei hohen Werten von $\hat{T}_{W}$ signifikant beobachtet, wenn die Amplitude der Kontrollmode einen für nichtlineare Wechselwirkungen relevanten Wert erreicht. Auch in den spannweitigen Spektren zur systematischen Variation der Heizleistung mit anderen Heizreihen sind Hinweise auf nichtlineare Wechselwirkungen zu erkennen. Näher diskutiert werden später nichtlineare Wechselwirkungen in dem aktuierten Fall, in dem auch die Störungsentwicklung entlang eines großen Profiltiefenbereichs untersucht wurde, siehe Abschnitt 7.5. 


\subsubsection{Zusätzlich angehobene instationäre Störungen}

Wie in Abschnitt 7.3.2 beschrieben, enthält die Anregung des Heizaktuators keine instationären Anteile, sondern besteht lediglich aus einer veränderten stationären Wandtemperaturverteilung. Eine beobachtbare Veränderung der instationären Störungsanteile in der Grenzschichtströmung kann also nur eine indirekte Folge der Aktuierung sein, wie beispielsweise eine Beeinflussung des Rezeptivitätsprozesses oder der Stabilitätseigenschaften der Grenzschicht. Der gewählte konstante Abstand für die Linienmessungen im Rahmen der systematischen Variation der Heizleistung entspricht nicht dem Wabstandstand des Amplitudenmaximums aller laufenden QSI. Daher sind die bei der Leistungsvariation beobachteten Effektivwerte instationärer Fluktuationen wahrscheinlich kleiner als das wandnormale Maximum. Der Einfluss der Heizaktuierung auf instationäre Fluktuationen wird anhand der wandnormal und spannweitig verteilten Hitzdrahtmessungen später detaillierter diskutiert, siehe Abschnitt 7.5.

Wie in Abschnitt 7.3.1 beschrieben, führt die Heizaktuierung zusätzlich zur erwünschten Wandtemperaturperiodizität auch zu einer Anhebung der spannweitig gemittelten Wandtemperatur. In Abschnitt 7.3.4 wurde die gemäß LST erwartete Destabilisierung der Grenzschicht bzgl. instationärer QSI beschrieben. Dabei wurde festgestellt, dass der bezüglich Frequenz und Wellenzahl maximale N-Faktor $N_{\max }$ sich gegenüber dem ungeheizten Fall bei $x_{c} / c=0.35$ und der Heizleistung $P=14 \mathrm{~W}$ im Mittel der unterschiedlichen Heizreihen um $\Delta N=0.12$ ändert, siehe Abschnitt 7.3.4. Um die beobachtete Änderung der Effektivwerte instationärer Fluktuationen mit der gemäß LST erwarteten Destabilisierung zu vergleichen, wurde diese Änderung ebenfalls als $\Delta N$ wie folgt ausgedrückt (siehe Definition des N-Faktors, Abschnitt 2.3):

$$
\Delta N=\ln \left(\frac{\operatorname{mean}_{y_{c}}\left(u_{s, r m s}\right)}{\operatorname{mean}_{y_{c}}\left(u_{s, r m s, 0}\right)}\right)
$$

Es handelt sich jeweils um den Effektivwert instationärer Fluktuationen im gesamten aufgelösten Frequenzbereich und $u_{s, r m s, 0}$ bezeichnet den Effektivwert im nicht-aktuierten Fall.

Diese experimentellen Werte für $\Delta N$ sind in Abbildung 7.4.9 abhängig von der entlang $x_{c}$ maximalen und spannweitig gemittelten Wandtemperaturanhebung dargestellt. Diese wurde mit der Wandtemperatur im unbeeinflussten Zustand entdimensionalisiert. Der höchste Wert auf der Abszisse wird für jede Heizreihe bei der Heizleistung $P=14 \mathrm{~W}$ erreicht. Man erkennt ins- 


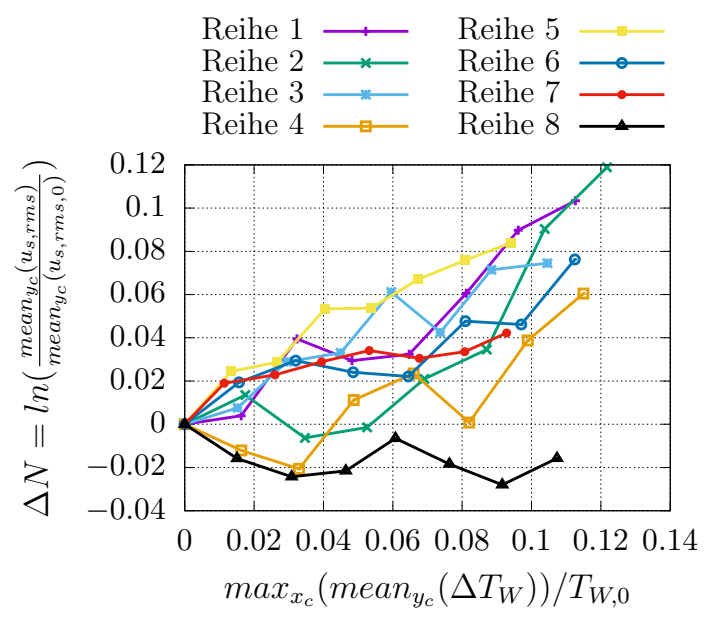

Abbildung 7.4.9: Änderungen im spannweitig gemittelten Effektivwert instationärer $u_{s}$-Fluktuationen bei der Leistungsvariation je Heizreihe

gesamt nur geringe Änderungen $\Delta N$ und für die meisten Heizreihen eine leichte Anhebung des spannweitig gemittelten Effektivwertes instationärer $u_{s}$-Fluktuationen. Die Anhebung liegt in allen Fällen noch unter dem Wert $\Delta N=0.12$. Es treten also keine systematischen Änderungen auf, welche die oben und in Abschnitt 7.3.4 beschriebene Destabilisierung der Grenzschicht durch die Anhebung der gemittelten Wandtemperatur überschreiten. Es werden also auch keine Hinweise auf eine deutliche Änderung der Rezeptivität der Grenzschicht bzgl. laufender QSI beobachtet.

\subsection{Transitionsszenario im heizaktuierten Fall}

Nachdem im vorangegangenen Abschnitt gezeigt werden konnte, dass mithilfe der spannweitig periodischen Wandheizung die stationäre QSI-Kontrollmode erfolgreich angeregt und durch Variation der Heizleistung in ihrer Amplitude gesteuert werden kann, soll nun überprüft werden, ob die Aktuierung zu einer Transitionsverzögerung gemäß der UFD-Methode führt. Aus früheren Untersuchungen im neu ausgelegten Prinzipexperiment mit einem pneumatischen Aktuator war ein Amplitudenniveau der Kontrollmode bekannt $\left(\hat{u}_{s} / q_{e} \approx 0.07\right.$ bei $x_{c} / c=0.3$, siehe [68]), welches für die nichtlineare Erzeugung einer MFD gemäß der UFD-Methode und zur Transitionsverzögerung ausreichte. Für die spannweitig periodische Wandheizung wurden Anregungsparameter gewählt, 
(a)

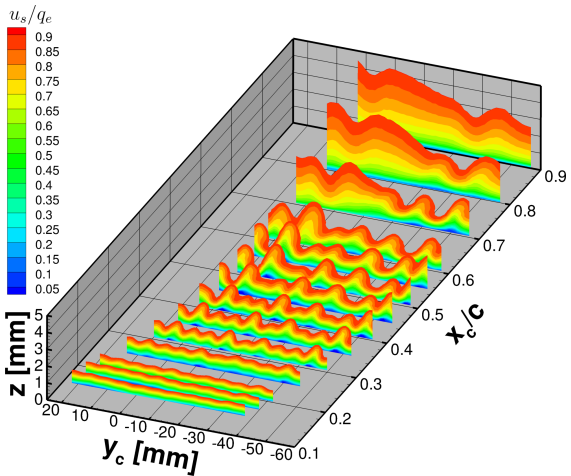

(b)

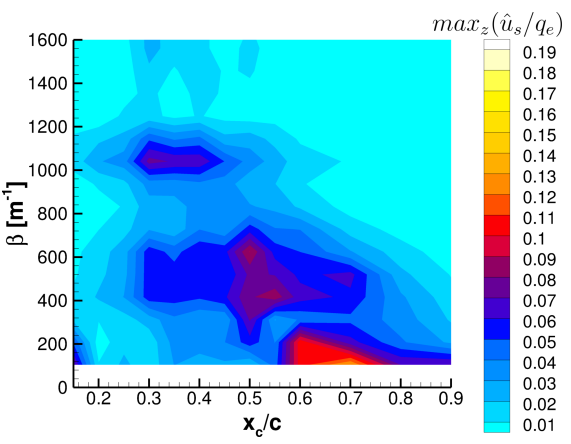

Abbildung 7.5.1: (a) Durch stationäre QSI deformiertes Grenzschichtströmungsfeld stromab der Heizaktuierung mit Heizreihe 3 und $P=18 \mathrm{~W}$ (b) Wandnormal maximal beobachtete Amplituden spannweitiger Periodizität der Grenzschichtdeformation

die zu einer ähnlichen Amplitude der Kontrollmode führten. Im Folgenden wird das Transitionsszenario stromab dieser stationären Anregung anhand dreidimensional verteilter Messungen repräsentativ für eine Beeinflussung mit diesem Anregungskonzept beschrieben. Des Weiteren wird auch erklärt, warum die Aktuierung nicht wie beim pneumatischen Aktuator zu einer Transitionsverzögerung führte.

Als Anregungsparameter wurde die Heizreihe 3 (mittlere Aktuierungsposition $x_{c} / c=0.066$ ) und die Heizleistung $P=18 \mathrm{~W}$ ausgewählt. Die spannweitig gemittelte, in Plattentiefenrichtung maximale Wandtemperaturanhebung dieser Heizreihe bei dieser Heizleistung beträgt $\Delta T_{W}=37.7 \mathrm{~K}$. Die erzeugte spannweitige Wandtemperaturperiodizität besitzt eine Amplitude von $\hat{T}_{W}=$ $3.7 \mathrm{~K}$.

\subsubsection{Die stationäre Störströmung}

Analog zum Fall bei ausgeschaltetem Heizaktuator (siehe Abb. 7.2.1(a)) ist für den aktuierten Fall in Abbildung 7.5.1(a) das durch stationäre QSI deformierte zeitlich gemittelte Grenzschichtströmungsfeld dargestellt. In farblichen Isokonturen ist die zeitlich gemittelte Geschwindigkeitskomponente $u_{s}$ dargestellt. Man erkennt deutlich die durch die Aktuierung induzierte 
spannweitige Periodizität mit $\lambda=6 \mathrm{~mm}$ zwischen $x_{c} / c=0.2$ und $x_{c} / c=0.4$. Im Vergleich zu Abb. 7.2.1(a) erkennt man insgesamt eine deutliche Änderung der stationären Störströmung.

Die am weitesten stromauf gelegenen Hitzdrahtmessungen fanden bei $x_{c} / c=$ 0.15 statt. In Abschnitt 7.3.1 wurde der Einfluss der spannweitig gemittelten Wandtemperaturanhebung auf Hitzdrahtmessungen besonders an der Position der Messungen zur Heizleistungsvariation $\left(x_{c} / c=0.35\right)$ und bei einer Heizleistung von $P=14 \mathrm{~W}$ abgeschätzt. Analog dazu wird für die mit $P=18 \mathrm{~W}$ betriebene Heizreihe 3 an der Plattentiefenposition $x_{c} / c=0.15$ noch eine wandnormal maximale und spannweitig gemittelte Temperaturanhebung in der Grenzschicht von $\Delta T=5.3 \mathrm{~K}$ erwartet. Dies stellt einen signifikanten Einfluss auf Hitzdrahtmessungen dar und bedeutet, dass die absoluten ausgewerteten Geschwindigkeiten bei Anwendung der Kalibrierung ohne Berücksichtigung dieses Einflusses einen zu geringen Betrag besitzen. Im Folgenden werden aber insbesondere spannweitige Störspektren und instationäre Störungen diskutiert, wobei dieser spannweitig mittlere stationäre Einfluss größtenteils irrelevant ist.

Mit den ausgewählten Betriebsparametern wird bei $x_{c} / c=0.066$ eine spannweitige Wandtemperaturperiodizität mit einer Amplitude von $\hat{T}_{W}=3.7 \mathrm{~K}$ erzeugt. Der maximale spannweitige Wandtemperaturunterschied zwischen Maximum und Minimum entspricht dort also $2 \cdot \hat{T}_{W}=7.4 \mathrm{~K}$. Analog zu der exemplarischen Diskussion in Abschnitt 7.3.1 für Reihe 1, wird für den hier betrachteten Fall bei $x_{c} / c=0.15$ im wandnormalen Maximum ein spannweitiger Temperaturunterschied von etwa $\Delta T=0.8 \mathrm{~K}$ erwartet, welcher stromab weiter abnimmt. Dieser Temperaturunterschied, dessen spannweitige Wellenlänge ebenfalls $\lambda=6 \mathrm{~mm}$ entsprechen sollte, ist also schon bei $x_{c} / c=0.15$ als gering zu bewerten. Im Folgenden wird gezeigt, dass die beobachtete Amplitude der spannweitigen Geschwindigkeitsperiodizität dieser Wellenlänge stromab zunimmt und nicht abklingt. Daher ist die Interpretation der gemessenen spannweitigen Geschwindigkeitsschwankungen als Folge einer stationären QSI gerechtfertigt.

In Abbildung 7.5.1(b) sind die wandnormal maximal beobachteten Amplituden $\hat{u}_{s}$ spannweitiger Periodizität der $u_{s}$-Komponente als Isokonturen abhängig von der spannweitigen Wellenzahl $\beta$ und der Plattentiefenkoordinate $x_{c} / c$ dargestellt. Die spektrale Auflösung der spannweitigen Spektren der $u_{s}$-Periodizität ist dabei durch die Länge des spannweitigen Messbereichs der Profilmessungen auf $\Delta \beta=104.05 \mathrm{~m}^{-1}$ eingeschränkt, siehe Abschnitt 6.3.1. 
Dabei sieht man angehobene Amplituden bei $\beta=1040 \mathrm{~m}^{-1}$, die etwa bei $x_{c} / c=0.3 \mathrm{ihr}$ Maximum erreichen und stromab wieder abnehmen. Diese spannweitige Wellenzahl entspricht etwa derjenigen der erfolgreich angeregten Kontrollmode $\left(\beta=1047 \mathrm{~m}^{-1}, \lambda=6 \mathrm{~mm}\right)$. Weiterhin sind im spannweitigen Spektrum der stationären Grenzschichtdeformation langwellige Anteile mit hoher Amplitude vertreten, besonders bei $\beta=416 \mathrm{~m}^{-1}$. Zusätzlich zu dem im Fall des ausgeschalteten Aktuators bereits beobachteten lokalen Maximums bei $\beta=416 \mathrm{~m}^{-1}$ ist ein weiteres lokales Maximum bei $\beta=624 \mathrm{~m}^{-1} \mathrm{zu}$ erkennen. Die Annahme liegt nahe, dass es sich hierbei um eine Folge einer nichtlinearen Wechselwirkung zwischen der Mode mit $\beta=416 \mathrm{~m}^{-1}$ und der angeregten Kontrollmode mit $\beta=1047 \mathrm{~m}^{-1}$ handelt, wodurch eine Mode mit $\beta=1047-416=631 \mathrm{~m}^{-1}$ in ihrer Amplitude angehoben wurde. Die Wellenzahl dieser Differenzmode entspricht im Rahmen der spektralen Auflösung nahezu der Wellenzahl des beobachteten lokalen Maximums bei $\beta=624 \mathrm{~m}^{-1}$ und liegt im Wellenzahlbereich der gemäß LST besonders stark integral angefachten stationären QSI. Schon bei der Diskussion der systematischen Variation der Heizleistung wurden im spannweitigen Spektrum der stationären Grenzschichtdeformation Hinweise auf nichtlineare Wechselwirkungen beobachtet, siehe Abschnitt 7.4.2.

\subsubsection{Entwicklung stationärer Störungen}

Die Entwicklung der Amplituden ausgewählter stationärer QSI-Moden ist im Vergleich zwischen dem Fall des ausgeschalteten Aktuators und dem aktuierten Fall in Abbildung 7.5.2 dargestellt. Dabei ist jeweils der Verlauf $\hat{u}_{s} / q_{e}=\hat{u}_{s, 0} / q_{e} \cdot e^{N}$ mit den zugehörigen N-Faktoren der LST-Untersuchung (Abschnitt 6.2.1) und jeweils passend gewähltem Wert für $\hat{u}_{s, 0}$ als gestrichelte Linie eingezeichnet. Für die Kontrollmode wurde dieser Verlauf in Abbildung 7.5.2(a) für die beiden Fälle mit zwei verschiedenen Werten für $\hat{u}_{s, 0}$ dargestellt, da durch die Aktuierung gerade eine höhere Anfangsamplitude der Kontrollmode erzielt wird. Die Amplitude der Kontrollmode mit $\beta=1040 \mathrm{~m}^{-1}$ (näherungsweise $\beta=1047 \mathrm{~m}^{-1}$ ) ist im aktuierten Fall deutlich gegenüber dem Fall ohne Aktuierung angehoben (siehe Abbildung 7.5.2(a)). Sie erreicht bei $x_{c} / c=0.3$ ihren maximalen Wert von $\hat{u}_{s} / q_{e}=0.074$. Im Fall ohne Aktuierung folgt die Entwicklung ihrer Amplitude etwa bis $x_{c} / c=0.5$ der gemäß LST erwarteten Entwicklung. Im aktuierten Fall weicht der Verlauf spätestens ab $x_{c} / c=0.3$ deutlich davon ab. 

(a) $\beta=1040 \mathrm{~m}^{-1}$
(b) $\beta=416 \mathrm{~m}^{-1}$

ohne Aktuierung Reihe $3, P=18 \mathrm{~W}$ $\hat{u}_{s, 0,1} / q_{e} \cdot e^{N} \ldots \ldots$ ohne Aktuierung $\hat{u}_{s, 0} / q_{e} \cdot e^{N}$

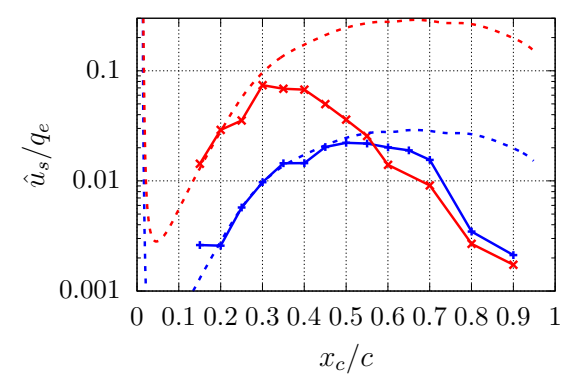

(c) $\beta=624 \mathrm{~m}^{-1}$

ohne Aktuierung — $\hat{u}_{s, 0} / q_{e} \cdot e^{N} \ldots .$. Reihe $3, P=18 \mathrm{~W}$

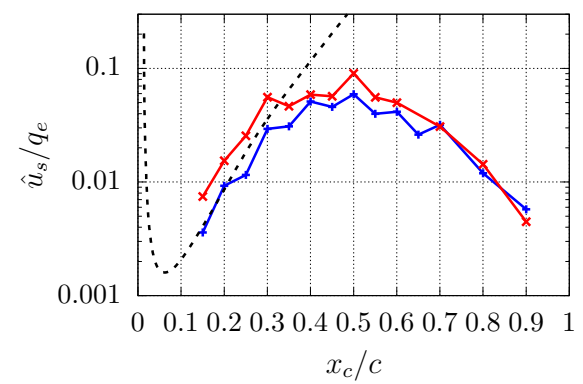

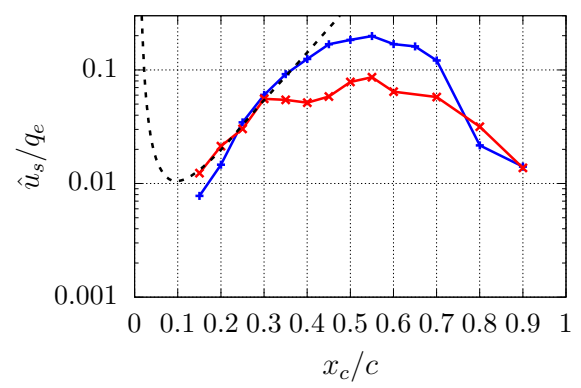

(d)

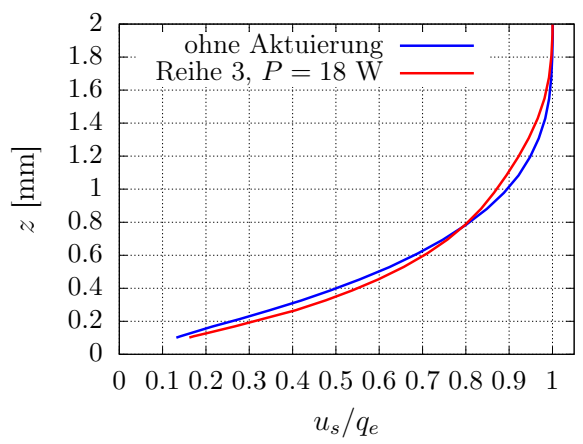

Abbildung 7.5.2: (a), (b) und (c) Wirkung der Aktuierung auf die Entwicklung der wichtigsten stationären QSI-Moden entlang $x_{c}$ (d) Spannweitig und zeitlich gemitteltes Grenzschichtprofil $u_{s} / q_{e}$ an der Position $x_{c} / c=0.3$ 
An derselben Plattentiefenposition erfährt das Amplitudenwachstum der bei ausgeschaltetem Heizaktuator dominierenden Mode mit $\beta=416 \mathrm{~m}^{-1}$ eine starke Steigungsänderung (Abbildung 7.5.2(b)) und erreicht stromab signifikant geringere Amplituden als ohne Aktuierung. Im Amplitudenmaximum entlang $x_{c}$ erreicht sie bei $x_{c} / c=0.55$ mit $\hat{u}_{s} / q_{e}=0.086$ einen weniger als halb so großen Wert wie im Fall ohne Aktuierung. Bis zur Position $x_{c} / c=0.3$ folgt die Amplitudenentwicklung dieser Mode im Fall ohne Aktuierung annähernd der gemäß LST erwarteten Entwicklung. Die Beobachtung der starken Steigungsänderung in der Amplitudenentwicklung der dominanten QSI-Mode deckt sich mit Fällen erfolgreicher Vordeformation der Grenzschicht gemäß der UFD-Methode [68, 111]. Wie in Abschnitt 2.3 beschrieben wurde, kann eine verringerte Amplitude stationärer QSI dann durch eine nichtlinear erzeugte Deformation der spannweitig mittleren Strömung mit stabilisierender Eigenschaft erklärt werden.

Bereits ab $x_{c} / c=0.15$ ist aber die Amplitude der spannweitigen Deformation mit $\beta=624 \mathrm{~m}^{-1}$ gegenüber dem ausgeschalteten Zustand leicht angehoben (Abbildung 7.5.2(c)), was wie oben beschrieben auf eine nichtlineare Wechselwirkung der angeregten QSI mit $\beta=1047 \mathrm{~m}^{-1}$ und der QSI mit $\beta=416 \mathrm{~m}^{-1}$ hindeutet. Letztere weist bei $x_{c} / c=0.3$ auch im Fall mit ausgeschaltetem Heizaktuator bereits eine Amplitude von knapp unter 6\% der Grenzschichtrandgeschwindigkeit $q_{e}$ auf. Ab $x_{c} / c=0.3$ endet im aktuierten Fall das Amplitudenwachstum der Mode mit $\beta=624 \mathrm{~m}^{-1}$ zunächst und die Amplitude bleibt bis zum finalen Umschlag im Vergleich zum Fall mit ausgeschaltetem Heizaktuator leicht erhöht. Damit weist auch diese Mode bei $x_{c} / c=0.3$ eine Steigungsänderung in ihrem Amplitudenwachstum entlang $x_{c}$ auf.

In Abbildung 7.5.2(d) ist das spannweitig gemittelte $u_{s}$-Grenzschichtprofil bei $x_{c} / c=0.3$, also der Plattentiefenposition der Steigungsänderung der QSI-Amplituden mit $\beta=416 \mathrm{~m}^{-1}$ und $\beta=624 \mathrm{~m}^{-1}$, für den Fall mit ausgeschaltetem Aktuator und im aktuierten Fall dargestellt. Das Profil ähnelt Grenzschichtprofilen, in denen numerisch eine stabilisierende MFD nachgewiesen wurde [111]. Das Profil ist in Wandnähe fülliger als im Heizaktuator-Referenzfall und zeigt etwa für $z>0.5 \mathrm{~mm}$ einen schwächeren Gradienten $\frac{\partial u_{s}}{\partial z}$ als stromab des ausgeschalteten Heizaktuators. Allerdings ist die Deformation des spannweitig gemittelten Profils im Vergleich mit Fällen erfolgreicher Upstream Flow Deformation schwach ausgeprägt. 


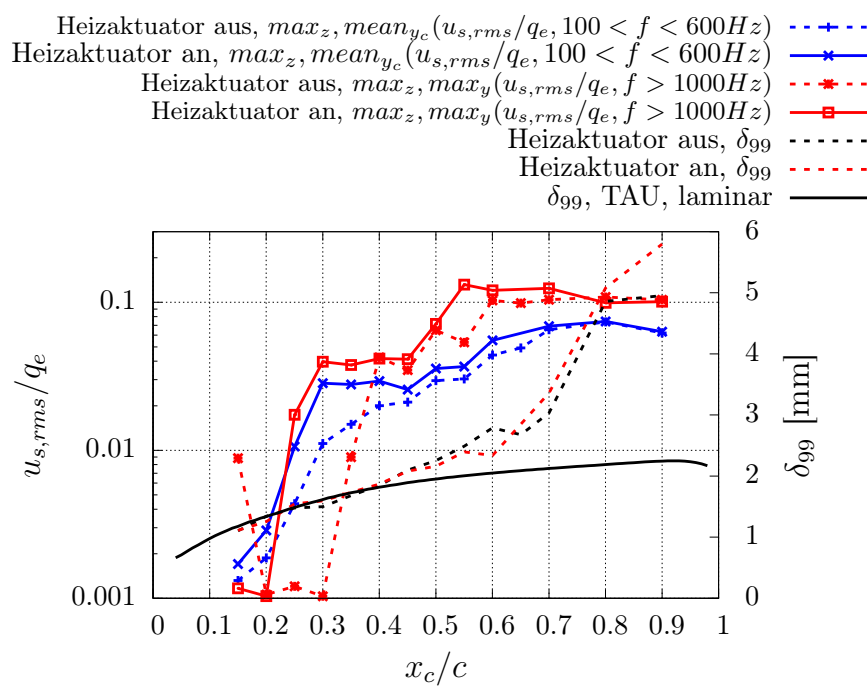

Abbildung 7.5.3: Wirkung der Aktuierung auf die beobachteten wandnormal maximalen Effektivwerte instationärer Fluktuationen und die Transitionslage

Es wurde durch die Aktuierung also eine signifikante Anhebung der Amplitude der Kontrollmode erreicht. Sie entspricht bei $x_{c} / c=0.3$ nahezu der Amplitude der Kontrollmode im Fall des pneumatischen Aktuators von Lohse et al. [68, 70], in dem die Aktuierung zu einer Transitionsverzögerung führte. Zusätzlich wurde beobachtet, dass stromab von $x_{c} / c=0.3$ die Amplitude der dominanten stationären QSI-Mode deutlich reduziert wurde.

\subsubsection{Entwicklung instationärer Störungen}

Die Entwicklung des wandnormalen Maximums des spannweitig gemittelten Effektivwertes von instationären $u_{s}$-Fluktuationen im Frequenzbereich $100<f<600 \mathrm{~Hz}$ ist in Abbildung 7.5.3 dargestellt. Diese Fluktuationen wurden im Referenzfall des Naseneinsatzes mit Druckbohrungen vor allem mit laufenden QSI assoziiert, siehe Abschnitt 6.3.2. Zusätzlich ist der Effektivwert hochfrequenter Fluktuationen mit Frequenzen $f>1000 \mathrm{~Hz}$ eingezeichnet, welche mit sekundären Instabilitäten oder turbulenten Fluktuationen assoziiert wurden. Davon unabhängig lässt sich die spannweitig mittlere Profiltiefenposition des Umschlags am stärkeren Aufdicken der Grenzschicht erkennen. Dazu ist die ermittelte Grenzschichtdicke $\delta_{99}$ im aktuierten Fall, im Fall mit 
ausgeschalteter Heizung und in der numerischen laminaren TAU-Simulation auf einer separaten Ordinatenachse eingezeichnet.

Der spannweitig gemittelte Effektivwert der Fluktuationen im Frequenzbereich $100 \mathrm{~Hz}<f<600 \mathrm{~Hz}$ ist im aktuierten Fall bereits bei $x_{c} / c=0.15 \mathrm{im}$ Vergleich zum Fall mit ausgeschaltetem Aktuator angehoben. Bei $x_{c} / c=0.3$ erreicht er einen Wert von knapp 3\% der Grenzschichtrandgeschwindigkeit, nähert sich aber stromab bei $x_{c} / c=0.45$ wieder dem Niveau des Falls ohne Aktuierung. In Abschnitt 7.4.3 wurde bei der systematischen Variation der Heizleistung keine signifikante Anhebung dieses Effektivwerts beobachtet. Die Messungen fanden dabei allerdings an einem festen Wandabstand statt, während hier der wandnormale Maximalwert dargestellt ist. Die Entwicklung des Effektivwerts korreliert örtlich entlang $x_{c}$ mit der Entwicklung der Kontrollmode (hier wegen der spektralen Auflösung mit $\beta=1040 \mathrm{~m}^{-1}$ diskutiert), was darauf hinweist, dass die Grenzschicht durch die künstlich angeregte stationäre Kontrollmode gegenüber instationären Störungen in diesem Frequenzbereich sekundär destabilisiert wurde. Das Annähern ihres spannweitig gemittelten Effektivwertes an das Niveau des Falls mit ausgeschaltetem Aktuator geschieht parallel zur entlang $x_{c}$ abnehmenden Amplitude der künstlich angeregten stationären Kontrollmode stromab von $x_{c} / c=0.3$. Der Erklärungsansatz einer sekundären Destabilisierung bietet sich auch für die beobachtete Entwicklung des Effektivwerts hochfrequenter Fluktuationen an.

Im aktuierten Fall ist schon ab $x_{c} / c=0.25$, also kurz stromauf der Position $x_{c} / c=0.3$, an der die stationäre Kontrollmode ihre maximale Amplitude erreicht, der Effektivwert der hochfrequenten Fluktuationen gegenüber dem Fall ohne Aktuierung angehoben. Stromab von $x_{c} / c=0.3$ verbleibt dieser aber bis $x_{c} / c=0.45$ bei einem nahezu konstanten Wert und erreicht ca. $0.05 c$ weiter stromauf als im Fall mit ausgeschaltetem Aktuator ein hohes Niveau von etwa $0.1 q_{e}$. Das transitionelle Aufdicken der Grenzschicht geschieht durch die Aktuierung leicht stromauf verschoben. Das starke Aufdicken der Grenzschicht geschieht wie im Fall ohne Aktuierung erst, nachdem die QSI-Mode mit $\beta=416 \mathrm{~m}^{-1}$ ihre maximale Amplitude erreicht hat (siehe Abbildung 7.5.2(b)). Eine Verschiebung der Transitionslage stromab, wie es das Ziel der UFD-Methode ist, konnte also trotz signifikanter Verminderung der Amplitude der dominanten stationären QSI nicht erreicht werden. 


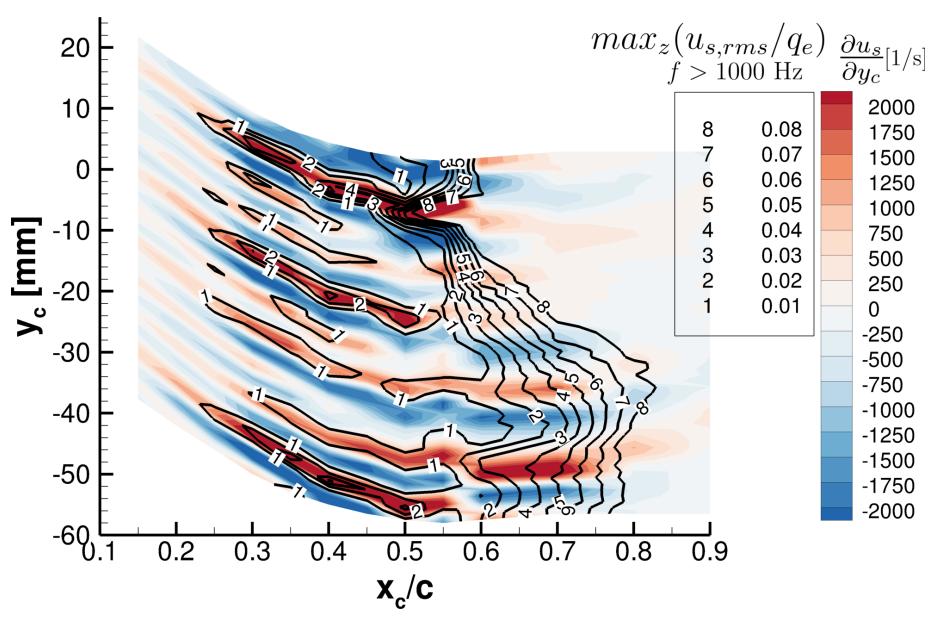

Abbildung 7.5.4: Spannweitige Gradienten der zeitlich gemittelten $u_{s}$-Komponente in farblichen Isokonturen und wandnormal maximale Effektivwerte instationärer $u_{s}$-Fluktuationen mit $f>1000 \mathrm{~Hz}$ in Isolinien

Der Erklärungsansatz der Sekundärinstabilität aufgrund hoher Amplituden der Kontrollmode wird im Folgenden für beide diskutierten Frequenzbereiche näher untersucht.

\subsubsection{Sekundärinstabilitäten}

Zunächst soll für hochfrequente instationäre Fluktuationen gezeigt werden, dass ihre spannweitigen Positionen mit der stationären Störströmung korrelieren. Als Überblick über die stationäre Störströmung ist in Abbildung 7.5.4 daher der spannweitige Gradient der zeitlich gemittelten $u_{s}$-Komponente je $x_{c}$-Position bei konstantem Wandabstand in farblichen Isokonturen dargestellt. Analog zu Abb. 6.3.6 und 7.2.6 wurde dafür je $x_{c}$-Position der Wandabstand ausgewählt, an dem der spannweitige Mittelwert der $u_{s}$-Komponente dem Wert mean $_{y_{c}}\left(u_{s} / q_{e}\right)=0.7$ am nächsten ist. Mit eingezeichnet ist der wandnormal maximale Effektivwert instationärer $u_{s}$-Fluktuationen mit $f>1000 \mathrm{~Hz}$ als Isolinien. Beim Vergleich mit Abbildung 7.2.6 fällt auf, dass die stationären QSI starke spannweitige $u_{s}$-Gradienten weiter stromauf erzeugen als im Fall mit ausgeschaltetem Heizaktuator. Spannweitig korreliert mit starken positiven spannweitigen Gradienten werden erhöhte Effektivwerte hochfrequenter Fluktuationen beobachtet, die auftreten, lange bevor die stationäre QSI-Mode mit $\beta=416 \mathrm{~m}^{-1}$ ihre maximale Amplitude erreicht 
bzw. bevor die Grenzschichtdicke verstärkt anwächst und die Grenzschicht final umschlägt. Zwischen $x_{c} / c=0.25$ und $x_{c} / c=0.4$ ist insbesondere eine spannweitige Korrelation dieser Effektivwerte hochfrequenter Fluktuationen mit der spannweitig periodischen stationären Grenzschichtdeformation mit $\lambda=6 \mathrm{~mm}$ aufgrund der künstlich angeregten Kontrollmode zu erkennen. Dies stützt den Erklärungsansatz der sekundären Destabilisierung durch die Kontrollmode. Spannweitig verteilt schnellen die Effektivwerte hochfrequenter Fluktuationen erst bei $x_{c} / c=0.6$ hoch.

Besonders interessant ist also, an welchen Positionen in der spannweitig und wandnormal vermessenen Ebene bei $x_{c} / c=0.3$ starke instationäre Fluktuationen beobachtet werden. Die spannweitige und wandnormale Verteilung des bei $x_{c} / c=0.3$ angehobenen Effektivwerts hochfrequenter Fluktuationen wird in Abbildung 7.5.5(a) als Isolinien dargestellt. Als Isokonturen ist der Wert des Gradienten $\frac{\partial u_{s}}{\partial z}$ dargestellt. Die angehobenen Effektivwerte werden insbesondere in Wandnähe beobachtet und weisen eine starke örtliche Korrelation mit hohen Werten dieses Gradienten auf (wie in Abschnitt 7.2 auch für den Fall des ausgeschalteten Heizaktuators beschrieben). Die Werte dieses Gradienten weisen hier aber eine klare spannweitige Periodizität mit einer Wellenlänge von $6 \mathrm{~mm}$ auf und stellen eine Deformation der stationären Grenzschichtströmung durch die künstlich angeregte Kontrollmode dar. Diese Beobachtungen passen zu den Erkenntnissen zu Sekundärinstabilitäten des Typs III (siehe [54]). Niedrige Werte des Effektivwertes von $u_{s}$-Fluktuationen mit $100 \mathrm{~Hz}<f<600 \mathrm{~Hz}$, welcher in Abb. 7.5.5(b) in Isolinien dargestellt ist, treten auch in größerem Wandabstand auf und sind dort ebenfalls moduliert mit der spannweitigen Wellenlänge der künstlich angeregten Kontrollmode. Die höheren Werte dieses Effektivwertes werden jedoch an denselben wandnahen Positionen beobachtet wie die erhöhten Effektivwerte hochfrequenter Fluktuationen. Dies weist darauf hin, dass an diesen Positionen ein breiter Frequenzbereich instationärer Fluktuationen sekundär destabilisiert ist.

Diese Sekundärinstabilitäten treten ab etwa $x_{c} / c=0.25$ bis zum finalen Umschlag stromab von $x_{c} / c=0.6$ auf. Kurz stromauf des finalen Umschlags wird wie schon im Fall des ausgeschalteten Heizaktuators ein Wechsel zu einem anderen Typ Sekundärinstabilität beobachtet. An einer einzelnen spannweitigen Position $\left(y_{c}=-5 \mathrm{~mm}\right)$ in der vermessenen Ebene wird bei $x_{c} / c=0.55$ ein besonders hoher spannweitiger $u_{s}$-Gradient beobachtet, der örtlich mit stark angehobenen Effektivwerten hochfrequenter Fluktuationen korreliert, siehe Abb. 7.5.6. Dies passt gut zur Beschreibung einer Sekundärinstabilität vom Typ I in der Literatur [54, 71, 72]. In der Darstellung in Abbildung 
(a) $f>1000 \mathrm{~Hz}$

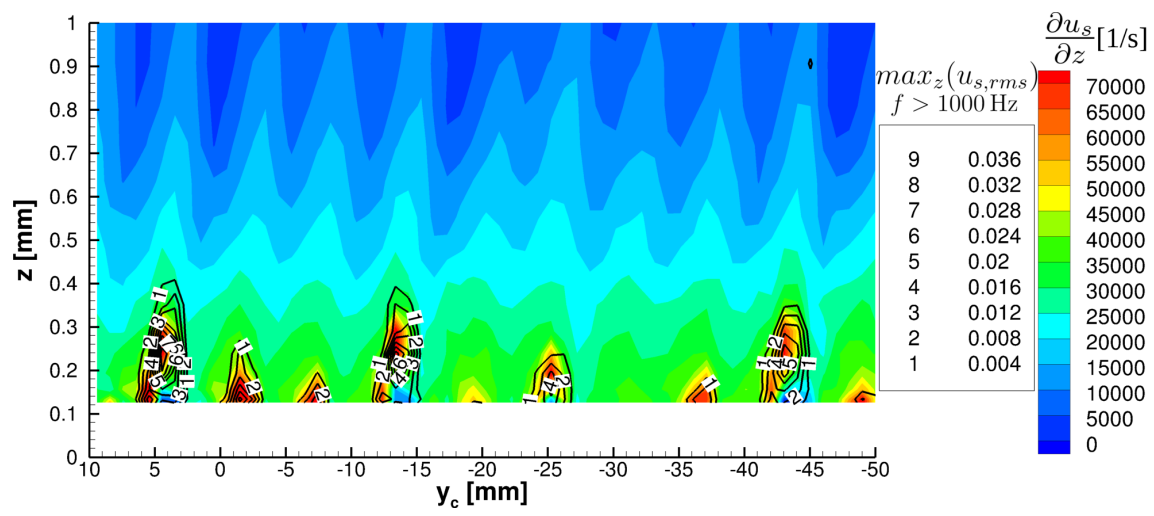

(b) $100 \mathrm{~Hz}<f<600 \mathrm{~Hz}$

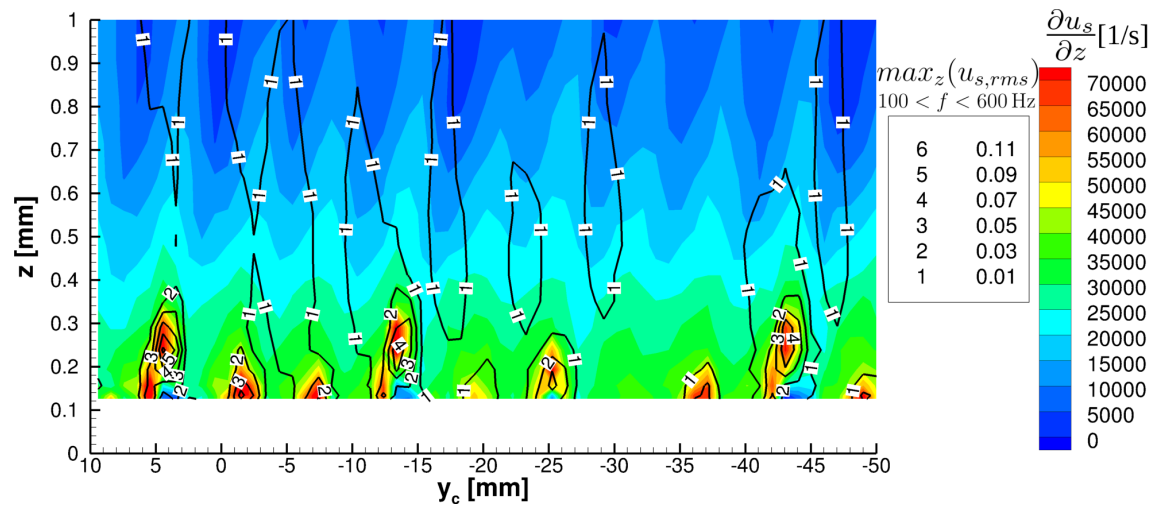

Abbildung 7.5.5: Werte des Gradienten $\frac{\partial u_{s}}{\partial z}$ bei $x_{c} / c=0.3$ in farblichen Isokonturen zur Visualisierung der wandnormalen Scherung durch die angeregten stationären QSI und die Effektivwerte von instationären $u_{s}$-Fluktuationen mit Frequenzen (a) von $f>1000 \mathrm{~Hz}$ und (b) von $100 \mathrm{~Hz}<f<600 \mathrm{~Hz}$ in Isolinien als Anzeichen für Sekundärinstabilitäten 


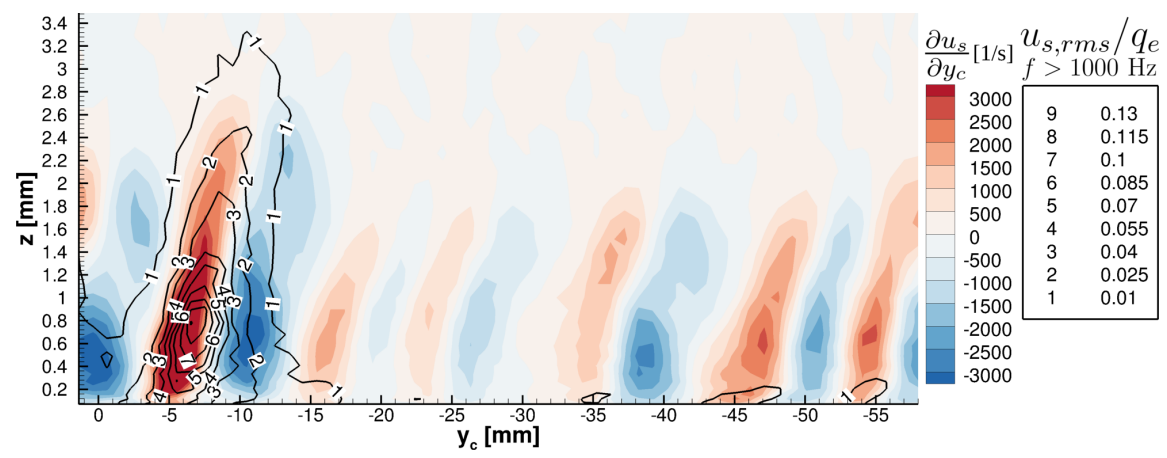

Abbildung 7.5.6: Werte des Gradienten $\frac{\partial u_{s}}{\partial y_{c}}$ bei $x_{c} / c=0.55$ in farblichen Isokonturen zur Visualisierung der spannweitigen Scherung und die Effektivwerte von instationären $u_{s}$-Fluktuationen mit Frequenzen $f>1000 \mathrm{~Hz}$ in Isolinien als Anzeichen für Sekundärinstabilitäten

7.5.4 erkennt man, dass maßgeblich von dieser spannweitigen Position die spannweitige Ausbreitung der angehobenen Werte des Effektivwerts hochfrequenter instationärer Fluktuationen beim finalen Umschlag ausgeht. In dem restlichen vermessenen spannweitigen Bereich treten bei $x_{c} / c=0.55$ nur die wandnahen Sekundärinstabilitäten vom Typ III mit vergleichsweise geringen Effektivwerten hochfrequenter Fluktuationen auf.

Wie anhand Abbildung 7.5.3 diskutiert wurde, wurde also im aktuierten Fall im Plattentiefenbereich $0.2<x_{c} / c<0.4$ eine Anhebung der Effektivwerte instationärer Fluktuationen in beiden diskutieren Frequenzbereichen gegenüber dem Fall mit ausgeschaltetem Aktuator beobachtet. Stromab von $x_{c} / c=0.4$ nähern sich die Effektivwerte der Fluktuationen beider Frequenzbereiche wieder dem Niveau des Falls mit ausgeschaltetem Aktuator an. Die beschriebene örtliche Korrelation der Effektivwerte mit der stationären Deformation der Grenzschicht durch die künstlich angeregte Kontrollmode bedeutet, dass die angehobenen Effektivwerte eine indirekte Folge der stationären Aktuierungswirkung und keine direkte instationäre Wirkung des Aktuators darstellen. Dadurch ist zu erwarten, dass mit jeder anderen stationären Anregungsmethode, welche die künstliche Kontrollmode in einem äquivalenten ReferenzSzenario und mit ähnlich großer Amplitude anregt, dasselbe beobachtet würde. In unveröffentlichten Auswertungen zu den Messungen mit dem pneumatischen Aktuator [68] wurden bei erfolgreicher Transitionsverzögerung tatsächlich ähnliche Hinweise auf solche Sekundärinstabilitäten beobachtet. 


\subsection{5 Überprüfung eines Transitionskriteriums}

Borodulin et al. [12, 13] haben in umfangreichen Untersuchungen zu querströmungsdominierter Transition in Fällen mit signifikanten Amplituden stationärer und laufender QSI ein empirisches Kriterium entwickelt, das die Transitionslage mit einem Schwellwert einer sog. ,kombinierten“ Störungsamplitude verknüpft. Dabei untersuchten sie Fälle mit verteilter Rauigkeit, also insbesondere ohne künstliche Anregung einer diskreten QSI-Mode. Die kombinierte Störungsamplitude ist definiert als die Summe der bilanzierenden Amplituden stationärer und instationärer Störungen [13]:

$$
A_{\text {ges }}=A_{\text {stat }}+A_{\text {instat }}
$$

Um diese bilanzierende Betrachtung auf den hier betrachteten Fall anzuwenden, wurde die Amplitude stationärer Instabilitäten aus dem wandnormal maximalen Effektivwert des deformierten spannweitigen Verlaufs der zeitlich gemittelten $u_{s}$-Komponente gewonnen:

$$
A_{\text {stat }}\left(x_{c}\right)=\sqrt{2} \cdot \max _{z} \sqrt{\frac{1}{Z} \sum_{i=1}^{Z}\left(u_{s}\left(x_{c}, y_{c, i}, z\right)-\bar{u}_{s}\left(x_{c}, z\right)\right)^{2}}
$$

Dabei entspricht $Z$ der Anzahl der spannweitigen Messpositionen und $\bar{u}_{s}$ dem spannweitigen Mittelwert der zeitlich gemittelten Geschwindigkeitskomponente $u_{s}$. Der Faktor $\sqrt{2}$ skaliert dabei den Effektivwert auf das Niveau einer Amplitude.

Die bilanzierende Amplitude instationärer Störungen wurde aus dem spannweitig gemittelten und wandnormal maximalen Effektivwert der instationären $u_{s}$-Fluktuationen gewonnen, in denen lediglich die spektralen Inhalte herausgefiltert wurden, welche signifikant mit dem Signal des Beschleunigungssensors korrelieren, siehe Abschnitt 5.1.

$$
A_{\text {instat }}\left(x_{c}\right)=\sqrt{2} \cdot \max _{z}\left(\operatorname{mean}_{y_{c}}\left(u_{s, r m s}\left(x_{c}, y_{c}, z\right)\right)\right)
$$

In Abbildung 7.5.7 ist die bilanzierende Amplitude stationärer Störungen, instationärer Störungen und die kombinierte Störungsamplitude $A_{\text {ges }}$ in ihrem Verlauf entlang der Plattentiefe $x_{c} / c$ im Vergleich für die Fälle mit aus- und eingeschaltetem Aktuator dargestellt. Zur Veranschaulichung der spannweitig gemittelten Transitionslage ist auch die mittlere Grenzschichtdicke $\delta_{99}$ auf einer separaten Ordinatenachse dargestellt. 


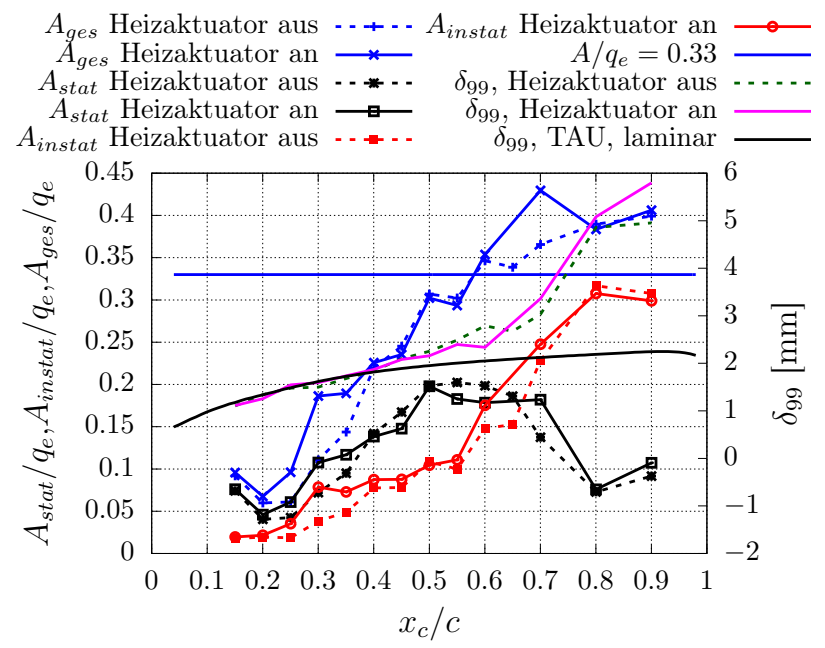

Abbildung 7.5.7: Die Rolle stationärer und instationärer Moden im Transitionsprozess anhand des Transitionskriteriums von Borodulin et al. [13]

Dabei ist zu erkennen, dass in beiden Fällen die kombinierte Störungsamplitude $A_{\text {ges }}$ den Schwellwert von Borodulin et al. [13] $A_{\text {ges }}=0.33 \cdot q_{e}$ zwischen $x_{c} / c=0.55$ und $x_{c} / c=0.6$ überschreitet. In dem Kriterium wird die Transitionslage als die Profiltiefenposition definiert, an der diejenigen hochfrequenten Sekundärinstabilitäten zuerst beobachtet werden, auf die kurz darauf der finale Umschlag folgt [13]. Im hier betrachteten Fall mit Heizaktuierung wurden auch schon bei $x_{c} / c=0.3$ Sekundärinstabilitäten mit hochfrequenten Fluktuationen beobachtet. Die Plattentiefenposition $x_{c} / c=0.55$ ist allerdings diejenige, ab der in Abbildung 7.5.4 das spannweitig verteilte Hochschnellen der Effektivwerte hochfrequenter instationärer Fluktuationen beobachtet wurde. Hochfrequente Geschwindigkeitsfluktuationen bei $x_{c} / c=0.55$ wurden anhand Abbildung 7.5.6 als Sekundärinstabilität vom Typ I interpretiert. Der damit eingeleitete finale Umschlag führt ab $x_{c} / c=0.7$ zum verstärkten Wachstum der Grenzschichtdicke. Somit scheint das von Borodulin et al. [13] eingeführte Transitionskriterium auch in den hier betrachteten Fällen anwendbar zu sein. Dies ist insbesondere deshalb interessant, weil durch die künstliche Anregung eine signifikant andere spektrale Verteilung stationärer Störungen vorliegt als bei den von Borodulin et al. untersuchten Fällen.

In der Bilanz muss man schlussfolgern, dass die Wirkung des Aktuators in diesem Fall hauptsächlich zu einer spektralen Umverteilung der Störströmung zu anderen Instabilitätsmoden geführt hat. Bei $x_{c} / c=0.3$ ist die 
Amplitude stationärer Störungen signifikant gegenüber dem nicht-aktuierten Fall angehoben, da in diesem Bereich die künstlich angeregte Kontrollmode ihre maximale Amplitude erreicht. In Abschnitt 7.5.1 wurde beschrieben, dass die stationäre Mode mit $\beta=624 \mathrm{~m}^{-1}$ aufgrund einer nichtlinearen Wechselwirkung in ihrer Amplitude angehoben wird. Gemeinsam mit der bis $x_{c} / c=0.55$ noch nicht ausreichend reduzierten Amplitude der Kontrollmode und der verbleibenden Amplitude der Mode mit $\beta=416 \mathrm{~m}^{-1}$ führt dies dazu, dass die bilanzierende Amplitude stationärer Störungen $A_{\text {stat }}$ im Vergleich zum Fall mit ausgeschaltetem Aktuator bis $x_{c} / c=0.55$ kaum reduziert ist. Parallel ist die bilanzierende Amplitude instationärer Störungen aufgrund von Sekundärinstabilitäten leicht gegenüber dem Fall bei ausgeschaltetem Aktuator angehoben, weshalb der Verlauf der kombinierten Amplitude $A_{\text {ges }}$ bei derselben Plattentiefe den Grenzwert des Kriteriums von Borodulin et al. erreicht. Nach dieser Bilanzbetrachtung ist hauptsächlich die nichtlineare Wechselwirkung der künstlich angeregten stationären Kontrollmode mit der QSI mit $\beta=416 \mathrm{~m}^{-1}$ dafür verantwortlich, dass die Aktuierung nicht zu einer Transitionsverzögerung geführt hat. Im Fall des pneumatischen Aktuators wurde eine solche ungünstige nichtlineare Wechselwirkung nicht beobachtet und es konnte eine Transitionsverzögerung erreicht werden [68, 70]. Dort enthielt die Rauigkeit der Modelloberfläche keine vergleichbaren langwelligen Anteile und die Wellenlänge und Amplitude der natürlich dominanten QSI-Mode waren durch eine künstliche Anregung mittels DRE fixiert. Es wurde also gezeigt, dass der Einsatz spannweitig periodischer Wandheizung grundsätzlich zur Transitionsverzögerung mit der UFD-Methode geeignet ist, dass aber die UFD-Methode sehr empfindlich gegenüber den Anfangsbedingungen des Transitionsszenarios und insbesondere dem Spektrum stationärer Störungen ohne Aktuierung ist. 


\section{Kapitel 8}

\section{Beeinflussung durch spannweitig periodische Rotation}

Das zweite Anregungskonzept, welches im Rahmen dieser Arbeit im Kontext der UFD-Methode zur Transitionsverzögerung untersucht wird, ist die Anregung durch spannweitig periodisch angeordnete und bündig in die Modelloberfläche integrierte rotierende Scheiben. Sie werden auch Drehteller genannt. Auch dieses Konzept ist in einem eigenen Naseneinsatz für die Aufnahmemöglichkeit der Modellplatte aufgebaut worden. Eine Hypothese zur Funktionsweise der Anregung wurde in Abschnitt 2.5.3 und die technische Realisierung in Abschnitt 4.5.2 beschrieben. Bei stillstehenden Drehtellern unterscheidet sich der Naseneinsatz nicht maßgeblich von dem Naseneinsatz des Referenzfalls, bis auf eine spannweitig periodische Störung geringer Amplitude durch die Rauigkeitseigenschaft der Drehteller selbst. Diese Störung befindet sich also ebenfalls an ihrer Position $x_{c} / c=0.1$ und hat die spannweitige Wellenlänge $\lambda=6 \mathrm{~mm}$. Aufgrund der einfachen Lagerung der Drehteller war leider die einwandfreie Rotation mit der gewünschten Drehzahl nicht für alle Drehteller gewährleistet. Daher musste der untersuchte spannweitige Bereich auf den Nachlauf der einwandfrei rotierenden Drehteller eingeschränkt werden. 
Anhand einer systematischen Variation der Drehzahl wird in Abschnitt 8.1 die künstliche Anregung stationärer QSI nachgewiesen und quantifiziert. Es werden zusätzlich angehobene instationäre Fluktuationen stromab der Anregung beobachtet. Im Folgenden werden in Abschnitt 8.1.3 im Wesentlichen Hinweise dafür präsentiert, dass es sich dabei um eine Folge technischer Einschränkungen der gewählten Realisierung und nicht um eine inhärente Eigenschaft einer Rotationsanregung handelt. In Abschnitt 8.2 wird für ausgewählte Anregungsparameter das Transitionsszenario stromab der verteilten Rotationsanregung charakterisiert und nachvollzogen, warum keine Transitionsverzögerung erreicht wurde.

\subsection{Systematische Variation der Anregungsamplitude}

Wie schon für die spannweitig periodische Wandheizung in Abschnitt 7.4, soll in diesem Abschnitt für die spannweitig periodische Rotationsanregung nachgewiesen werden, dass es mit diesem Anregungskonzept möglich ist, eine stationäre QSI-Kontrollmode mit wohldefinierter spannweitiger Wellenlänge anzuregen und ihre Amplitude zu steuern. Dies wird anhand einer systematischen Variation der Rotationsfrequenz $f_{\text {rot }}$ und spannweitigen Linienmessungen im Nachlauf bei $x_{c} / c=0.3$ und bei konstantem Wandabstand gezeigt. Als Wandabstand wurde wieder derjenige gewählt, an dem die maximale Amplitude der Kontrollmode beobachtet wurde. Wie im Fall der Heizaktuierung wurde zur nachträglichen Bestimmung des Wandabstandes für jede Linienmessung der spannweitige Mittelwert der zeitlich gemittelten $u_{s}$-Komponente mit dem Wert aus der spannweitig und wandnormal verteilt vermessenen Ebene an derselben Profiltiefenposition verglichen. Aus diesem Vergleich ergibt sich als Mittelwert aller Linienmessungen zur systematischen Variation der Rotationsfrequenz ein Wandabstand von $z=0.55(5) \mathrm{mm}$, wobei der angegebene Fehler der Standardabweichung entspricht.

Die einzeln regelbare Rotationsfrequenz der Drehteller ermöglicht neben der Untersuchung der spannweitig periodischen Anregung auch die Untersuchung einer Anregung durch einen einzeln rotierenden Drehteller während die restlichen Drehteller still stehen. Eine spannweitig periodische Anregung durch mehrere gleichzeitig mit derselben Drehzahl rotierende Drehteller besitzt die diskrete spannweitige Periodizität der ausgewählten Kontrollmode mit $\lambda=6 \mathrm{~mm}$. Im Folgenden wird begrifflich zwischen einer verteilten und einer Einzelanregung unterschieden. Neben dem Nachweis der künstlichen Anre- 
gung der Kontrollmode wird für die verteilte Anregung geprüft, welchen Einfluss der Drehsinn auf die spannweitig periodische Anregung hat. Des Weiteren wird gezeigt, dass auch die Anregung einer stationären QSI-Mode mit der doppelten Wellenlänge der ausgewählten Kontrollmode möglich ist.

\subsubsection{Künstliche Anregung stationärer QSI: verteilte Anregung}

\section{Details zur spannweitig periodischen Rotationsanregung}

Die zur Strömung exponierten Drehteller induzieren in der in Abschnitt 2.5.3 beschriebenen Anregungshypothese durch die Haftbedingung eine wandparallele Störgeschwindigkeit. Es ist zu erwarten, dass sie eine Anfangsstörung darstellt, aus der bei spannweitig periodischer Anregung im Rezeptivitätsprozess eine stationäre QSI-Mode, nämlich die ausgewählte Kontrollmode entsteht. Als Maß für die Stärke der Anregung wird unter anderem direkt die Drehzahl bzw. Rotationsfrequenz $f_{\text {rot }}$ verwendet, welche durch die Regelung konstant gehalten wird. Somit werden auch bei der Rotationsaktuierung nominell stationäre Zustände untersucht. Die maximale lokal induzierte Geschwindigkeit parallel zur Modelloberfläche entspricht aufgrund der Haftbedingung der Bahngeschwindigkeit des Drehtellers abhängig vom Radius $r$ :

$$
q_{\text {Bahn }}=2 \pi r f_{\text {rot }}
$$

Die Bahngeschwindigkeit verschwindet im Zentrum des Drehtellers und erreicht am äußeren Rand $r=R=1 \mathrm{~mm}$ ihren Maximalwert $q_{B}:=$ $\max _{r}\left(q_{B a h n}\right)$. Da die Details des Rezeptivitätsprozesses unbekannt sind, soll im Folgenden diese maximale Bahngeschwindigkeit $q_{B}$ des Drehtellers, entdimensionalisiert mit der Grenzschichtrandgeschwindigkeit $q_{e}$ am Ort der Anregung $\left(x_{c} / c=0.1\right)$, als charakteristische Größe für die Anregungsamplitude verwendet werden. Im Rahmen dieser Arbeit wird angenommen, dass sie in linearer Art und Weise die Amplitude der initialen Störung, welche für die Anregung einer stationären QSI relevant ist, quantifiziert. In Tabelle 8.1.1 sind die Betriebsparameter Drehzahl, Rotationsfrequenz und $q_{B}$ für die im Folgenden beschriebenen Untersuchungen aufgeführt.

Aufgrund des geringen zur Verfügung stehenden Bauraums und aus Kostengründen musste auf eine aufwendige mechanische Lagerung der Drehteller verzichtet werden. Sie sind gemeinsam mit den Motoren in einer Position fixiert, in der sie bei Rotation mit dem jeweiligen Drehtellerkragen am Gegen- 


\begin{tabular}{cccc} 
Drehzahl $[1 / \mathrm{min}]$ & $f_{\text {rot }}[1 / \mathrm{s}]$ & $q_{B}[\mathrm{~m} / \mathrm{s}]$ & $q_{B} / q_{e}$ \\
\hline 10000 & 166.67 & 1.05 & 0.05 \\
15000 & 250 & 1.57 & 0.07 \\
20000 & 333.33 & 2.09 & 0.09 \\
25000 & 416.67 & 2.62 & 0.11 \\
30000 & 500 & 3.14 & 0.14 \\
35000 & 583.33 & 3.67 & 0.16 \\
40000 & 666.67 & 4.19 & 0.18 \\
45000 & 750 & 4.71 & 0.2 \\
50000 & 833.33 & 5.24 & 0.23 \\
55000 & 916.67 & 5.76 & 0.25 \\
60000 & 1000 & 6.28 & 0.27 \\
65000 & 1083.33 & 6.81 & 0.3
\end{tabular}

Tabelle 8.1.1: Charakteristische Parameter für die Anregung mit dem Rotationsaktuator

stück im Aktuatoreinsatz schleifen (siehe Abschnitt 4.5.2 und besonders Abb. 4.5.4(b)). Bei langwierigem Betrieb gerieten einige der Drehteller in einen Zustand, in dem das Drehmoment des jeweiligen Motors nicht ausreichte, um die Rotationsbewegung mit der gewünschten Drehzahl aufrecht zu erhalten. Auch bei erhöhter Leistung des Motors blieben einige Drehteller immer wieder stehen, was die rasterförmigen Hitzdrahtmessungen einer verteilten Anregung einschränkte. Für die Untersuchungen zur verteilten Anregung der Kontrollmode wurde der spannweitige Messbereich an der stromab gelegenen Messposition $x_{c} / c=0.3$ so ausgewählt, dass sie sich genau im Nachlauf der längsten ununterbrochenen spannweitigen Reihe von frei und mit der gewünschten Drehzahl rotierenden Drehtellern befand. Um den Nachlauf dieses spannweitigen Bereichs bei der Messposition $x_{c} / c=0.3$ korrekt zu bestimmen, wurden die Drehteller am jeweiligen spannweitigen Rand dieses Bereichs einzeln betrieben und ihr stationärer Einfluss stromab spannweitig lokalisiert.

\section{Die Anregung der ausgewählten stationären Kontrollmode}

Zur Zuordnung wurden die einzelnen Drehteller und ihre zugehörigen Motoren in positiver spannweitiger Richtung $+y_{c}$ aufsteigend nummeriert. Zur Untersuchung der Amplitude der angeregten Kontrollmode wurden die Drehteller 9 bis 29 jeweils bei zeitlich konstanter und für alle Drehteller identischer Rotationsfrequenz $f_{\text {rot }}$ gleichsinnig rotierend betrieben. In Aufsicht auf die 
(a)

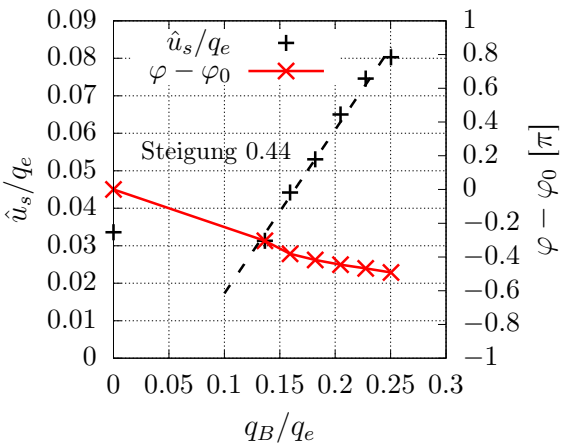

(b)

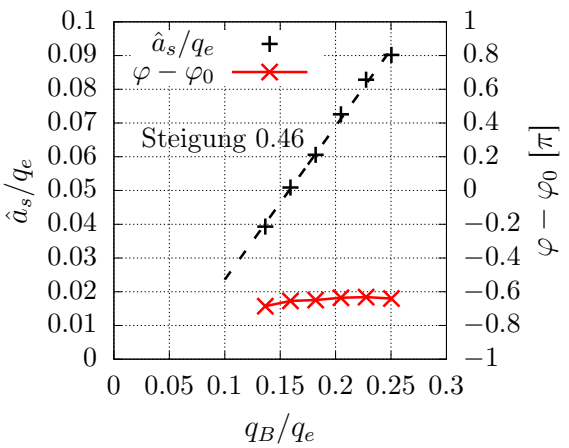

Abbildung 8.1.1: (a) Amplitude $\hat{u}_{s}$ der angeregten Kontrollmode $(\lambda=6 \mathrm{~mm})$ bei $x_{c} / c=0.3$ bei verteilter Rotationsanregung und die zugehörige Phasenlage $\varphi$ der spannweitigen Periodizität (b) Analog dazu Amplitude $\hat{a}_{s}$ und die Phasenlage $\varphi$ der spannweitigen Periodizität in $a_{s}$

Modellplatte wurde dabei zunächst der Drehsinn gegen den Uhrzeigersinn (GUZ) gewählt. Dieser Drehsinn entspricht im Rahmen der in Abschnitt 2.5.3 dargestellten Rezeptivitätshypothese dem Drehsinn der erwarteten gleichsinnig rotierenden Längswirbel. Aus dem Spektrum der spannweitigen Periodizität der gemessenen $u_{s}$-Komponente wurde die Amplitude bei der Wellenlänge $\lambda=6 \mathrm{~mm}$ ausgewertet. Dabei wurde, wie in Abschnitt 7.4.1 beschrieben, der spannweitige $u_{s}$-Verlauf vor der spektralen Zerlegung auf eine Strecke von $16 \cdot \lambda=96 \mathrm{~mm}$ eingeschränkt. Des Weiteren wurde bei der spektralen Zerlegung eine Von-Hann-Fensterfunktion verwendet.

Aufgrund der oben beschriebenen Einschränkungen bzgl. der Drehteller und damit der Reproduzierbarkeit der Anregung wurde der Fokus der Messungen auf die Untersuchung der Anregungseffizienz stationärer QSI analog zu Abschnitt 7.4.1 gelegt. Daher wurden Linienmessungen nur für Rotationsfrequenzen durchgeführt, für die bei einem einzeln betriebenen Drehteller bereits eine leichte Änderung des zeitlich gemittelten Grenzschichtströmungsfeldes beobachtet wurde. Das Verhalten der Kontrollmoden-Amplitude bei kleinen Anregungsamplituden, bei denen für den Heizaktuator teilweise eine Abnahme beobachtet wurde, wurde also nicht untersucht.

Die ausgewertete Amplitude $\hat{u}_{s}$ der angeregten Kontrollmode und die zugehörige Phasenlage $\varphi$ sind in Abbildung 8.1.1(a) abhängig von $q_{B} / q_{e}$ dargestellt. Bei letzterer wurde die Phasenlage $\varphi_{0}$ der Periodizität im nicht-aktuierten 
Zustand abgezogen. Dabei lässt sich ein linearer Anstieg der gemessenen QSIAmplitude mit $q_{B} / q_{e}$ feststellen. An den Verlauf von $\hat{u}_{s} / q_{e}\left(q_{B} / q_{e}\right)$ wurde durch Minimierung der Summe der Fehlerquadrate eine Ausgleichsgerade angepasst, welche gestrichelt in Abb. 8.1.1(a) eingezeichnet ist. Die Steigung der Geraden beschreibt die Effizienz der Störungseinbringung und beträgt 0.44(3). Dabei nimmt die gemessene Phasenlage der Kontrollmode kontinuierlich ab, was einer spannweitigen Verschiebung der Wirbelachsen in $+y_{c^{-}}$ Richtung entspricht. Die Tatsache, dass die Phasenlage der Kontrollmode durch die verteilte Anregung eine deutliche Änderung erfährt, motiviert die Annahme, dass auch bei der Rotationsaktuierung eine Überlagerung der angeregten Kontrollmode aufgrund von Rauigkeiten und derjenigen aufgrund der Rotation stattfindet, ähnlich wie beim Heizaktuator (siehe Abschnitt 7.4.1). Im Gegensatz zum Heizaktuator ist allerdings davon auszugehen, dass die Modelloberfläche nur an der Profiltiefenposition $x_{c} / c=0.1$ eine relevante Störung mit der spannweitigen Wellenlänge der Kontrollmode enthält. Die spannweitig periodisch angeordneten Drehteller stellen selbst eine signifikante Rauigkeit dar, welche bereits im ausgeschalteten Zustand des Aktuators eine relevante Anregung und stromab eine relevante Amplitude $\hat{u}_{s}$ bei $\lambda=6 \mathrm{~mm}$ bewirkt.

Wie in Abschnitt 7.4.1 beschrieben, wurde auch hier die Aktuierungswirkung in den gemessenen spannweitigen $u_{s}$-Verläufen näherungsweise isoliert, indem $u_{s, 0}\left(y_{c}\right)$ stromab des ausgeschalteten Aktuators vom jeweiligen Verlauf im aktuierten Zustand $u_{s}\left(y_{c}\right)$ abgezogen wurde. Der so erzeugte Verlauf $a_{s}\left(y_{c}\right)=u_{s}\left(y_{c}\right)-u_{s, 0}\left(y_{c}\right)$ enthält also näherungsweise nur die isolierte Wirkung der Aktuierung und nicht die Wirkung der Rauigkeiten. Aus diesen Verläufen bei unterschiedlicher Anregungsamplitude $q_{B} / q_{e}$ wurde die Amplitude $\hat{a}_{s}$ bei $\lambda=6 \mathrm{~mm}$ und die Phasenlage $\varphi$ der zugehörigen Periodizität ausgewertet. Zur Darstellung wurde wieder die Phasenlage $\varphi_{0}$ der $u_{s}$-Periodizität im ausgeschalteten Fall abgezogen. Diese Amplitude und Phasenlage sind in Abbildung 8.1.1(b) abhängig von der Anregungsamplitude $q_{B} / q_{e}$ dargestellt. Es ergibt sich eine linear wachsende Amplitude $\hat{a}_{s}$ mit steigender Anregungsamplitude $q_{B}$ und eine nahezu konstante Phasendifferenz zum Fall mit ausgeschaltetem Aktuator von gemittelt -0.65(1) $\pi$. Diese nahezu konstante Phasendifferenz unterstützt die Annahme, dass die beobachtete Änderung der Phasenlage der Periodizität in $u_{s}\left(y_{c}\right)$ eine Konsequenz der Überlagerung der Rotationsanregung mit einer Rauigkeitsanregung ist. Wieder wurde eine Ausgleichsgerade an den Amplitudenverlauf durch Minimierung der Summe der Fehlerquadrate angepasst, welche gestrichelt eingezeichnet 
ist. Die Steigung dieser Ausgleichsgeraden entspricht mit dem Wert 0.46(2) nahezu der Steigung des Verlaufs von $\hat{u}_{s} / q_{e}\left(q_{B} / q_{e}\right)$.

Die Oberfläche des Rotationsaktuator-Naseneinsatzes unterscheidet sich zur Oberfläche des Naseneinsatzes im Referenzfall (siehe Abschnitt 6.3.1) im Wesentlichen nur durch die Anwesenheit der Drehteller. In diesem Referenzfall wurden bei $x_{c} / c=0.1$ sowie weiter stromab keine relevanten QSIAmplituden bei $\lambda=6 \mathrm{~mm}\left(\beta=1047 \mathrm{~m}^{-1}\right)$ gemessen. Daher scheint die Annahme sinnvoll, dass die relevante Anregung durch Rauigkeit bei dieser spannweitigen Wellenlänge im Fall des Rotationsaktuators überwiegend an derselben Plattentiefenposition wie die Anregung durch Rotation geschieht. Die beobachtete mittlere Phasendifferenz von $-0.65(1) \pi$ entspricht einer spannweitigen Distanz von $1.95 \mathrm{~mm}$, was annähernd dem Durchmesser der Drehteller entspricht. Dies könnte darauf hinweisen, dass die elementaren Längswirbel, welche von der Rotation und von der Rauigkeitseigenschaft der Drehteller angeregt werden, entgegengesetzten spannweitigen Positionen am Rand des Drehtellers entspringen. Da Informationen über die zeitlich gemittelte Rauigkeitseigenschaft der Drehteller im rotierenden Zustand fehlen, ist diese Interpretation aber noch spekulativ. Zum Verständnis des Rezeptivitätsmechanismus sind wie für die Heizanregung auch für die Rotationsanregung tiefergehende numerische Untersuchungen nötig.

\section{Einfluss des Drehsinns bei verteilter Anregung}

Aufgrund der beschriebenen experimentellen Einschränkungen musste für die Untersuchung zum Einfluss des Drehsinns der spannweitig untersuchte Bereich auf den Nachlaufbereich der einwandfrei funktionierenden Drehteller weiter eingeschränkt werden. Für die Untersuchung des Drehsinn-Einflusses wurden die Drehteller 11 bis 24 jeweils bei konstanter Rotationsfrequenz $f_{\text {rot }}$ in gleichsinniger Drehrichtung betrieben. Der so aktuierte spannweitige Bereich umfasst mit $\Delta y_{c}=13 \lambda$ eine Strecke von $\Delta y_{c}=78 \mathrm{~mm}$. Eine systematische Variation der Rotationsfrequenz wurde für die ausgewählten Drehteller und beide Drehsinne wiederholt. Der Wechsel des Drehsinns wurde dabei durch Umpolen der Betriebsspannung der Motoren erreicht. Wegen der Einschränkungen bzgl. der gewünschten konstanten Rotationsfrequenz wurden für jeden Drehsinn nur drei Rotationsfrequenzen untersucht.

Die ausgewerteten Amplituden $\hat{u}_{s} / q_{e}$ und zugehörigen Phasenlagen $\varphi$ der künstlich angeregten Kontrollmode mit $\beta=1047 \mathrm{~m}^{-1}$ sind in Abbildung 8.1.2(a) in Abhängigkeit von der Anregungsamplitude $q_{B} / q_{e}$ dargestellt. Bei 
(a)

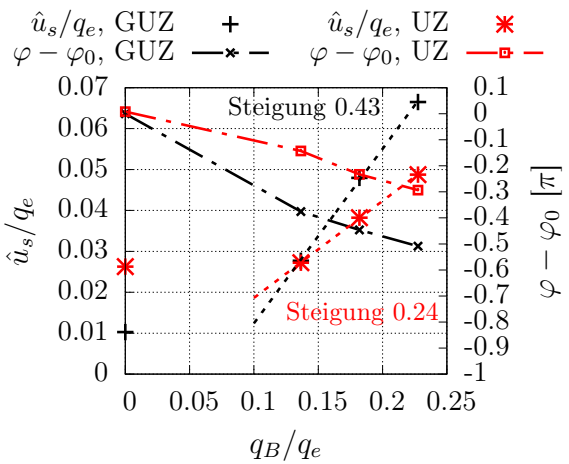

(b)

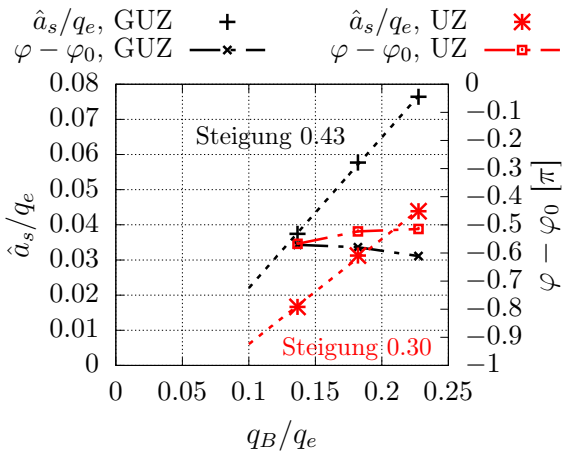

Abbildung 8.1.2: Einfluss des Drehsinns der gleichsinnigen verteilten Rotationsanregung auf die beobachtete Amplitude der Kontrollmode $(\lambda=6 \mathrm{~mm})$ und ihre Phasenlage (a) Amplituden $\hat{u}_{s}$ und zugehörige Phasenlagen (b) Amplituden $\hat{a}_{s}$ und zugehörige Phasenlagen

der Phasenlage wurde der Wert $\varphi_{0}$ im nicht aktuierten Zustand abgezogen. Bei den Messungen wurde zunächst die Variation der Anregungsamplitude bei Betrieb im Drehsinn GUZ untersucht. Bevor im Anschluss dieselbe Variation bei umgekehrtem Drehsinn, also im Uhrzeigersinn bei Aufsicht auf die Drehteller (UZ) untersucht wurde, wurde die Linienmessung bei ausgeschaltetem Aktuator wiederholt. Wie in Abb. 8.1.2(a) zu erkennen, ergab sich im ausgeschalteten Zustand $\left(q_{B}=0\right)$ eine andere Amplitude, während die Phasenlage nahezu perfekt reproduziert wurde. Dies deutet darauf hin, dass die Amplitude der spannweitig periodischen Störung durch Rauigkeit, welche die Drehteller im ausgeschalteten Zustand darstellen, auch von der Position abhängt, in der die einzelnen Drehteller nach dem Abschalten anhalten. Die Phasenlage der periodischen Anregung bei der Wellenlänge $\lambda=6 \mathrm{~mm}$ durch diese Rauigkeit ist allerdings geometrisch vorgegeben und ändert sich daher nicht.

Für beide Drehsinne wird für die aktuierten Fälle $\left(q_{B}>0\right)$ wieder eine lineare Steigerung der Kontrollmodenamplitude bzgl. der Anregungsamplitude $q_{B} / q_{e}$ beobachtet. An beide aktuierten Verläufe wurden Ausgleichsgeraden durch Minimierung der Summe der Fehlerquadrate angepasst. Diese Ausgleichsgeraden sind durch gestrichelte Linien in Abb. 8.1.2(a) dargestellt und ihre Steigung ist im Diagramm angegeben. Bei jeweils nur drei Messwerten im aktuierten Zustand ist die quantitative Steigung der linearen Amplituden- 
steigerung nicht sehr zuverlässig. Im Fall des Drehsinns GUZ wurde die Steigung, die bei der Anregung mit den Drehtellern 9-29 bestimmt wurde, mit einem Wert von 0.43(2) nahezu perfekt reproduziert. Es lässt sich an der größeren Steigung auch erkennen, dass die Rotationsaktuierung bei dem Betrieb der Drehteller im Drehsinn GUZ effizienter die Kontrollmode anregt als im Drehsinn UZ.

Die Phasenlage verschiebt sich für beide Drehsinne ins Negative, was einer spannweitigen Verschiebung der Längswirbelachsen in $+y_{c}$-Richtung entspricht. Bei der in Abschnitt 2.5.3 beschriebenen Rezeptivitätshypothese würde man zwischen den beiden Drehsinnen einen signifikanten Unterschied in der Phasenlage erwarten, welcher nahezu der gegenphasigen Lage entspricht. Die reine Störströmung einer stationären QSI-Mode besteht aus spannweitig periodisch gegensinnig rotierenden Wirbeln mit einem Wirbelachsenabstand von $\lambda / 2$, aus denen in Überlagerung mit der stationären Grundströmung spannweitig periodisch gleichsinnig rotierende Längswirbel mit Wirbelachsenabstand $\lambda$ verbleiben. Bei Umkehrung des Drehsinns würde die Rotationsanregung im Rahmen der vorgestellten Hypothese jeweils den spannweitig um $\lambda / 2$ verschobenen Teil der Störströmung anregen. Man würde also bei gegensätzlichem Drehsinn der Drehteller einen Phasenunterschied der experimentell angeregten Kontrollmode von $\pi$ erwarten. Der beobachtete Phasenunterschied zwischen den im GUZ-Fall und UZ-Fall angeregten QSIModen beträgt aber stattdessen lediglich ca. $0.2 \pi$, was einem Zehntel einer Periode und somit $\Delta y_{c}=0.6 \mathrm{~mm}$ entspricht.

Wie in Abschnitt 7.4.1 und in diesem Abschnitt bzgl. Abbildung 8.1.1(b) beschrieben, wurde auch für die Untersuchung des Drehsinns die Aktuierungswirkung von der Rauigkeitswirkung getrennt und die $\hat{a}_{s}$-Amplituden bei der Kontrollmoden-Wellenlänge sowie die zugehörigen Phasenlagen ausgewertet. Ihr jeweiliger Verlauf abhängig von $q_{B} / q_{e}$ ist in Abbildung 8.1.2(b) dargestellt. Für die Darstellung der Phasenlagen wurde wieder die Phasenlage $\varphi_{0}$ der Periodizität in $u_{s}$ abgezogen. In den $\hat{a}_{s}$-Verläufen erkennt man wieder, dass die GUZ-Anregung effektiver zu sein scheint als die UZ-Anregung. Die Phasendifferenz $\varphi-\varphi_{0}$ zum ausgeschalteten Fall beträgt im Fall mit der kleinsten untersuchten Anregungsamplitude für beide Drehsinne etwa $-0.57 \pi$. Mit zunehmendem Wert für $q_{B}$ ändert sie sich wie im vorangegangenen Abschnitt nur geringfügig im Vergleich zur beobachteten Änderung der Phasenlage in der $u_{s}$-Periodizität (siehe Abb. 8.1.2(a)). Für den Drehsinn GUZ wird bei der stärksten Anregung mit $q_{B} / q_{e}=0.23$ wieder ein ähnlicher Wert dieser Phasendifferenz erreicht wie im vorigen Abschnitt mit Drehteller 9-29. Für 
den Drehsinn UZ wird eine Phasendifferenz von $-0.51 \pi$ erreicht. Die Phasenlagen der durch gegenseitigen Drehsinn angeregten QSI unterscheiden sich nur wenig. Dies ist eine weitere Beobachtung, welche wie oben erläutert die in Abschnitt 2.5.3 beschriebene Anregungshypothese eher nicht unterstützt.

\section{Verteilte Anregung mit doppelter Wellenlänge}

Die individuelle Regelung der einzelnen Motoren ermöglicht es im Gegensatz zum Heizaktuator auch, stationäre QSI mit ganzzahligen Vielfachen der ausgelegten spannweitigen Wellenlänge anzuregen. Um dies zu demonstrieren, wurde nur jeder zweite Drehteller (ungerade nummeriert) mit einer konstanten Rotationsfrequenz von $f_{\text {rot }}=833.33 \mathrm{~Hz}$ bzw. mit einer Anregungsamplitude von $q_{B} / q_{e}=0.23$ in Rotation versetzt, während alle geradzahlig nummerierten Drehteller still standen.

Aus einer Linienmessung bei $x_{c} / c=0.3$ und konstantem Wandabstand wurde das spannweitige Spektrum stationärer QSI bestimmt, welches im Vergleich zum Fall ohne Aktuierung in Abbildung 8.1.3 dargestellt ist. Der spannweitige Messbereich mit der Länge $99.99 \mathrm{~mm}$ wurde dabei so gewählt, dass sie den Nachlauf der Drehteller 21, 23, 25, 27, 29, 31, 33, 35 und 37 abdeckte. Dabei war die freie Rotation des Drehtellers 25 wegen einer Fehlfunktion eingeschränkt. Seine gemittelte Rotationsfrequenz und Anregungsamplitude betrugen nur $f_{\text {rot }}=524(220) \mathrm{Hz}$ bzw. $q_{B} / q_{e}=0.14(6)$ mit jeweils deutlich erhöhter zeitlicher Schwankungsbreite des jeweiligen Wertes während der Messung im Vergleich zu den anderen Drehtellern. Dadurch handelt es sich bei der untersuchten Anregung nicht um eine rein monochromatische Anregung.

In Abb. 8.1.3 ist aber im aktuierten Fall eindeutig ein zusätzliches lokales Maximum im spannweitigen Spektrum bei einer Wellenzahl zu erkennen, welche im Rahmen der spektralen Auflösung der halben Wellenzahl der Kontrollmode entspricht, also $\beta=524 \mathrm{~m}^{-1}$. Somit wurde eine stationäre QSIMode mit der Wellenlänge $\lambda=12 \mathrm{~mm}$ erfolgreich angeregt.

\subsubsection{Stationäre Grenzschichtdeformation durch Einzelanregung}

Zusätzlich zur spannweitig verteilten Anregung durch möglichst viele Drehteller bei gleicher Rotationsfrequenz wurde auch die Anregung durch den Betrieb eines einzelnen Drehtellers untersucht, während alle anderen Drehteller still 


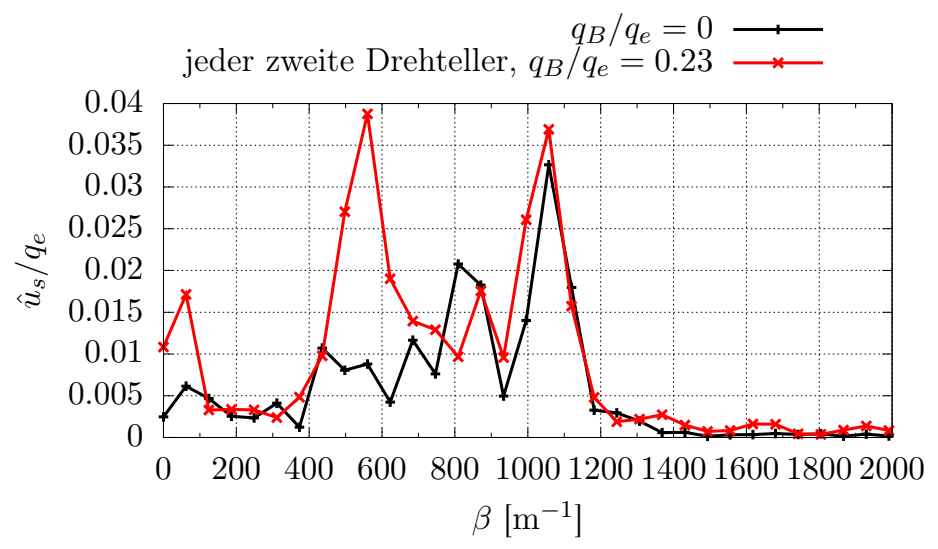

Abbildung 8.1.3: Spannweitiges Spektrum stationärer QSI an der Position $x_{c} / c=$ 0.3 bei Betrieb jedes zweiten Drehtellers mit konstanter Drehzahl im Vergleich zum Fall mit ausgeschalteten Motoren

standen. Dazu wurden ebenfalls Linienmessungen wie im obigen Abschnitt beschrieben bei $x_{c} / c=0.3$ und bei konstantem Wandabstand durchgeführt. Es wurde derselbe Wandabstand gewählt wie bei den Messungen zur verteilten Anregung der Kontrollmode.

Der spannweitige Messbereich wurde so gewählt, dass die spannweitig maximale Deformation der stationären Grenzschichtströmung etwa in der Mitte des vermessenen Bereichs lag. Aus den Messungen wurde die Aktuierungswirkung, wie in den Abschnitten 7.4.1 und 8.1.1 bereits beschrieben, von der Rauigkeitswirkung getrennt. Es wurde jeweils der spannweitige Verlauf der $u_{s^{-}}$ Komponente ausgewertet und der spannweitige Verlauf der $u_{s}$-Komponente im Fall des stillstehenden Drehtellers $\left(u_{s, 0}\right)$ abgezogen. Die Linienmessung bei stillstehendem Drehteller enthält die stationäre Grenzschichtdeformation aufgrund der Oberflächenrauigkeit des Modells und insbesondere der Rauigkeitseigenschaft der Drehteller selbst. Der spannweitige $a_{s}$-Verlauf stellt dann idealisiert die isolierte stationäre Wirkung der Einzelanregung dar.

Zur Veranschaulichung ist in Abbildung 8.1.4 die isolierte Wirkung $a_{s}\left(y_{c}\right)$ exemplarisch für Drehteller 9 und verschiedene Anregungsamplituden bei abgezogenem spannweitigen Mittelwert $\bar{a}_{s}$ dargestellt. Dabei ist klar die eingebrachte stationäre Deformation zu erkennen, welche bei hohen Werten von $q_{B}$ eine signifikante Stärke erreicht. Die Amplitude der durch die Einzelanregung 


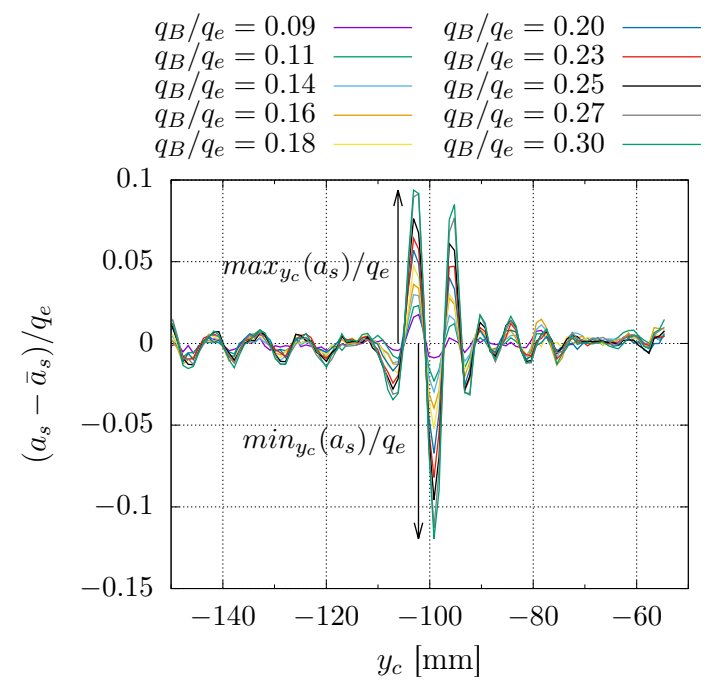

Abbildung 8.1.4: Exemplarisch isolierte Aktuierungswirkung $a_{s}\left(y_{c}\right)$ bei einzeln rotierendem Drehteller 9 mit jeweils unterschiedlicher Anregungsamplitude $q_{B}$

eingebrachten stationären Deformation wird nun wie folgt definiert:

$$
\hat{a}_{s}^{*}=\frac{1}{2}\left(\max _{y_{c}}\left(a_{s}\right)-\min _{y_{c}}\left(a_{s}\right)\right)
$$

Die so definierte Amplitude ist für verschiedene einzeln betriebene Drehteller in Abhängigkeit von der Anregungsamplitude $q_{B} / q_{e}$ in Abbildung 8.1.5 im Vergleich dargestellt. Dabei erkennt man für die Drehteller 9, 11 und 16, dass die eingebrachte Deformation ab einer gewissen Mindestanregungsamplitude einem gemeinsamen linearen Zusammenhang mit der Anregungsamplitude $q_{B} / q_{e}$ folgt. Mithilfe eines Algorithmus zur Minimierung der Summe der Fehlerquadrate wurde eine Ausgleichsgerade für $q_{B} / q_{e}>0.1$ an diese drei Amplitudenverläufe angepasst. Ihre Steigung quantifiziert die Effizienz der Einbringung der dimensionslosen Amplitude $\hat{a}_{s}^{*} / q_{e}$ durch die dimensionslose Anregungsamplitude $q_{B} / q_{e}$ und hat den Wert 0.51(2) mit dem Anpassungsfehler als angegebene Unsicherheit. Drehteller 28 zeigt hingegen ein anderes Verhalten. Die eingebrachte Deformation ist bei geringen Anregungsamplituden $q_{B} / q_{e}$ deutlich höher als bei den anderen drei Drehtellern. Es wird ebenfalls ein annähernd linearer Zusammenhang beobachtet, dessen Steigung allerdings einen geringeren Wert annimmt. Mithilfe lichtmikroskopischer Un- 


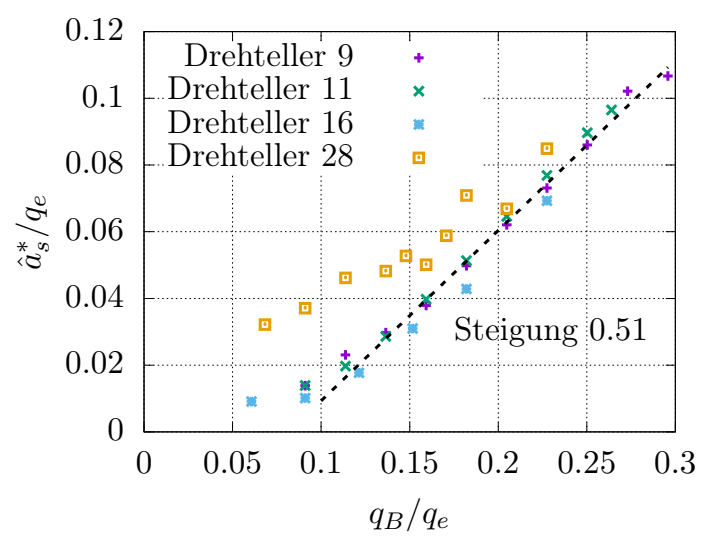

Abbildung 8.1.5: Amplitude der stationären Grenzschichtdeformation durch eine Einzelanregung für unterschiedliche Drehteller (Definition siehe Gleichung 8.1.2), gemessen bei $x_{c} / c=0.3$

tersuchungen im Stillstand wurde kein dafür klar relevanter Unterschied zwischen Drehteller 28 und den anderen diskutierten Drehtellern festgestellt.

Wie schon bei der Auswertung zur verteilten Anregung lässt sich auch für die Einzelanregung das Spektrum der spannweitigen $a_{s}$-Deformation aus den spannweitigen Verläufen bestimmen. Die Anregung an einer einzelnen spannweitigen Position bedeutet eine spektral breit verteilte Anregung bzgl. ihrer spannweitigen Wellenzahl $\beta$. Da der Wandabstand des Amplitudenmaximums stationärer QSI von ihrer Wellenzahl $\beta$ abhängt, entspricht die ausgewertete Amplitude anderer spektraler Anteile als der Kontrollmode nicht dem jeweiligen Amplitudenmaximum. In Abbildung 8.1.6 sind die Amplituden der spannweitigen $a_{s}$-Periodizität exemplarisch für Drehteller 9 abhängig von ihrer Wellenzahl $\beta$ und für alle untersuchten Anregungsamplituden $q_{B} / q_{e}$ dargestellt. Zum Vergleich ist die Verteilung der integralen Anfachung $\hat{a}_{s, L S T}=A_{0} e^{N(\beta)}$ gemäß der LST-Untersuchung bei der Messposition $x_{c} / c=0.3$ und passend gewählter wellenzahlunabhängiger Anfangsamplitude $A_{0}$ dargestellt. Diese Anfangsamplitude ist so gewählt, dass die Verteilung integraler Anfachung möglichst gut mit der bei der höchsten Anregungsamplitude gemessenen Amplitudenverteilung übereinstimmt.

Dabei erkennt man, dass die gemessene Amplitudenverteilung näherungsweise gut mit der theoretisch erwarteten Amplitudenverteilung einer bzgl. des spannweitigen Spektrums breit verteilten Anregung mit wellenzahlunabhängiger Anfangsamplitude übereinstimmt. Die Anregung durch einen einzelnen 


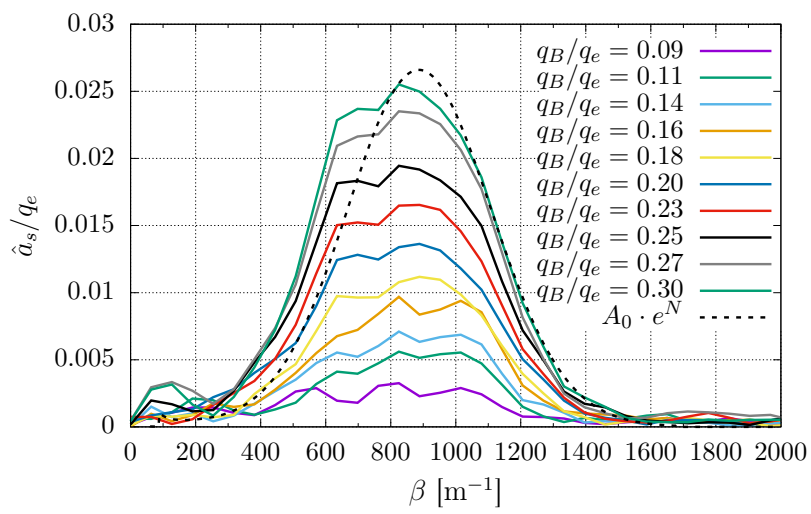

Abbildung 8.1.6: Amplituden $\hat{a}_{s} / q_{e}$ der spannweitigen $a_{s}$-Periodizität in Abhängigkeit von ihrer spannweitigen Wellenzahl $\beta$, exemplarisch für Drehteller 9 und verschiedene Anregungsamplituden $q_{B} / q_{e}$

Drehteller stellt eine örtlich stark lokalisierte Anregung dar, welche im Wellenzahlenraum einer breit verteilten Anregung entspricht und welche stromab durch die selektive Anfachung der Grenzschicht zu der bei der Messposition gemessenen Amplitudenverteilung führt.

\subsubsection{Instationäre Störungen stromab der Einzelanregung}

Stromab der rotierenden Drehteller und auch stromab eines einzeln rotierenden Drehtellers wurden trotz einwandfreier Rotation mit zeitlich konstanter Rotationsfrequenz auffällige instationäre Geschwindigkeitsschwankungen in der Grenzschicht beobachtet. Die Rotationsanregung basiert nach der in Abschnitt 2.5.3 beschriebenen Hypothese auf einem durch die Haftreibung am rotierenden Drehteller induzierten Drehimpuls und einem dadurch erzeugten Längswirbel. Eine solche Anregung wäre bei einem idealen Drehteller rein stationär, sofern die Rotationsfrequenz konstant, die Oberfläche des Drehtellers unendlich glatt oder perfekt rotationssymmetrisch und eine jegliche Auslenkung des Drehtellers ausgeschlossen wäre. Eine zusätzliche Anregung instationärer Störungen in der Grenzschicht kann einen Einsatz der Rotationsanregung im Rahmen der UFD-Methode unmöglich machen, wie es z.B. bisher bei Plasmaaktuatoren der Fall war (siehe [105]). Daher soll im Folgenden vor allem untersucht werden, ob es sich bei den angehobenen instationären Schwankungen um eine inhärente Eigenschaft der Rotationsanregung oder 
um die Konsequenz technischer Einschränkungen der hier untersuchten Realisierung handelt. Da unabhängige Messungen zu instationären Phänomenen an den Drehtellern nicht durchgeführt wurden, kann keine direkte Zuordnung zwischen instationärer Anregung und instationären Störungen stromab erfolgen. Daher werden in diesem Abschnitt die Eigenschaften der beobachteten instationären Schwankungen stromab von unterschiedlichen jeweils einzeln betriebenen Drehtellern detailliert untersucht und verglichen. Bevor die Beobachtungen näher diskutiert werden, sollen im Folgenden zunächst mögliche Ursachen für instationäre Störungen bei der Rotationsanregung vorgestellt werden.

\section{Mögliche Ursachen für die Anregung instationärer Störungen}

Bei den hier untersuchten realen Drehtellern wurde die Oberflächenrauigkeit reduziert, indem die Drehteller bei konstanter Drehzahl rotiert und in Kontakt mit Schleifpapier gebracht wurden. Trotzdem besitzen die Drehteller eine nicht völlig rotationssymmetrische Oberflächentopologie, die durch die Rotation gegenüber der Strömung eine zeitlich periodisch veränderte Anregung darstellt. Die Periodizität dieser instationären Anregung durch Oberflächenrauigkeit besäße eine diskrete Anregungsfrequenz, welche der Rotationsfrequenz $f=$ $f_{\text {rot }}$ entspricht.

Des Weiteren besitzen die verwendeten Elektromotoren einen Kommutator aus drei Segmenten, welcher wie in Abschnitt 4.5.2 beschrieben, die Regelung der Motordrehzahl ermöglicht. Die drei Segmente des Kommutators führen dazu, dass pro Umdrehung der durch den Motor fließende Strom dreimal unterbrochen wird. Während eines Bruchteils der Rotationsperiode wird kein Drehmoment erzeugt. Die Trägheit der rotierenden Bauteile des Motors inklusive des an der Achse fixierten Drehtellers sorgt dafür, dass die Rotation nicht unterbrochen wird. Allerdings bedeutet dies, dass trotz der auf einen konstanten Wert geregelten Rotationsfrequenz die Rotationsgeschwindigkeit des Drehtellers zeitlich nicht perfekt konstant ist. Das Ausmaß dieses Effektes wurde nicht untersucht. Der kurzfristige Einbruch der Rotationsgeschwindigkeit dreimal pro Periode könnte aber eine instationäre Anregung bei $f=3 f_{\text {rot }}$ erklären.

Die Motorachse, an welcher der zur Strömung exponierte Drehteller fixiert ist, besitzt Spiel in ihrer Lagerung innerhalb des Motors. Daher kann der Rotationsbewegung von Achse und Drehteller eine Präzessionsbewegung oder eine vertikale Auslenkung überlagert sein. Wie in Abschnitt 4.5.2 beschrieben, 
wurden die Drehteller mit einem Kragen versehen, der durch den schleifenden Kontakt mit der im Naseneinsatz vorgesehenen Gegenseite eine vertikale Auslenkung des Drehtellers in die Grenzschicht hinein verhindern soll. Bei leicht schräger Einbaulage ist es jedoch möglich, dass der Kragen räumlich nur teilweise im Kontakt mit der Gegenseite ist und diese Maßnahme nicht wie beabsichtigt wirkt. Zudem ist eine vertikale Auslenkung auch nur zur Oberseite beschränkt, eine Auslenkung zur Unterseite ist wegen des Spiels in der Achsenlagerung denkbar. Die beschriebenen Bewegungen könnten also ebenfalls eine instationäre Anregung in der darüber strömenden Grenzschicht darstellen.

Wie in Abbildung 4.5.4(b) dargestellt, befindet sich zwischen dem Drehteller und dem Motorgehäuse ein Hohlraum. Kommt es in dem dort eingeschlossenen Luftvolumen zu einer Druckschwankung, könnte diese durch die Spalte zwischen Drehteller und Modell die Grenzschichtströmung erreichen und dort wiederum eine instationäre Anregung darstellen. Betrachtet man diesen Hohlraum idealisiert als Helmholtzschen Resonator und schätzt mit den gegebenen geometrischen Dimensionen die Resonanzfrequenz ab, erhält man eine Frequenz von mehreren Kilohertz. Damit ist die Frequenz oberhalb des Frequenzbereichs, der für eine Anregung laufender QSI relevant ist (siehe Abschnitt 6.2.2).

Bei den bisher beschriebenen denkbaren Mechanismen für eine instationäre Anregung von Grenzschichtinstabilitäten wurden Phänomene direkt am Verbund des Motors, der Achse und des Drehtellers diskutiert. Die dort erzeugten Störungen stellen eine initiale Anregung dar, welche als instationäre Instabiliätsmoden in die Grenzschicht einkoppeln und stromab angefacht sein können. Ein weiterer Erklärungsansatz ist ein rein aerodynamischer in der Grenzschicht oberhalb des Drehtellers. Die Rotation des Drehtellers führt an seinem äußeren Rand in radialer Richtung teilweise zu einem starken Geschwindigkeitsgradienten zwischen dem vom Drehteller beschleunigten Fluid und der unbeeinflussten Grenzschichtströmung. Solche starken Gradienten können in der Grenzschicht oberhalb des Drehtellers zusätzliche Instabilitäten erzeugen und so für stromab beobachtete instationäre Fluktuationen verantwortlich sein. Dieses Phänomen wäre eine inhärente Eigenschaft der Rotationsanregung.

Mit der Variation der Drehzahl wird neben der Stärke des eingebrachten Drehimpulses auch die charakteristische Frequenz vieler der beschriebenen denkbaren Quellen für instationäre Störungen verändert. Bei mechanischen 


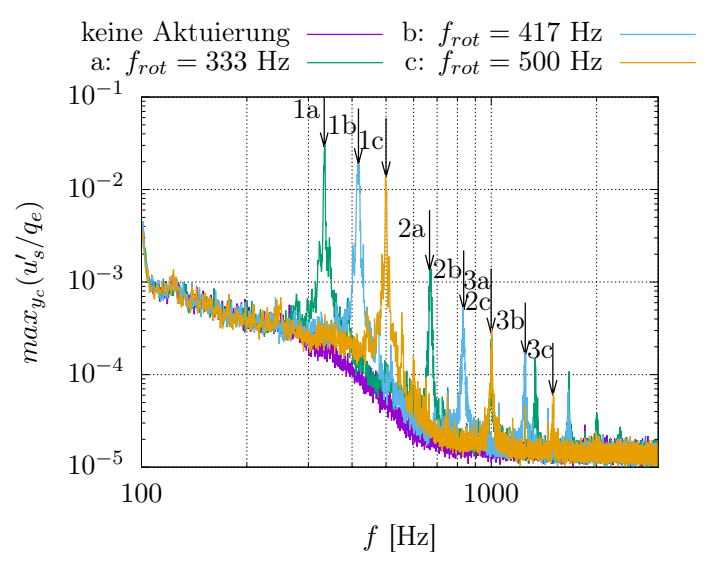

Abbildung 8.1.7: Spannweitig maximale Amplituden instationärer $u_{s}$-Schwankungen in Abhängigkeit ihrer Frequenz $f$ für unterschiedliche Rotationsfrequenzen $f_{\text {rot }}$ einer Einzelanregung durch den Motor 9

Anregungsmechanismen, welche auf einer Auslenkung der Drehtelleroberfläche mit dieser Frequenz basieren, ist zu erwarten, dass auch die Stärke der Anregung mit der Frequenz zunimmt. Ist die Auslenkung der Drehtelleroberfläche selbst frequenzunabhängig, so wächst die dadurch eingebrachte Beschleunigung des darüberliegenden Fluids mit der Rotationsfrequenz des Drehtellers. Also wäre die Amplitude der instationären Anregung auch in diesem Fall abhängig von $f_{\text {rot }}$.

\section{Instationäre Störungen bei ganzzahligen Vielfachen der Rotationsfrequenz}

Im Folgenden werden die instationären Störungen stromab der Einzelanregung anhand derselben Linienmessungen diskutiert, welche in Abschnitt 8.1.2 bzgl. der eingebrachten stationären Deformation der Grenzschicht ausgewertet wurden. Es handelt sich dabei um Linienmessungen bei $x_{c} / c=0.3$, konstantem Wandabstand und jeweils konstanter Rotationsfrequenz $f_{\text {rot }}$ eines einzeln betriebenen Drehtellers bei $x_{c} / c=0.1$.

Zunächst sollen nun die offensichtlichsten instationären Störungen stromab der Rotationsanregung diskutiert werden: Solche bei Frequenzen, die einem ganzzahligen Vielfachen der Rotationsfrequenz $f_{\text {rot }}$ entsprechen. In Abbildung 8.1.7 ist exemplarisch für Drehteller 9 das Spektrum der spannweitig maximal beobachteten Amplituden instationärer $u_{s}$-Fluktuationen abhängig 


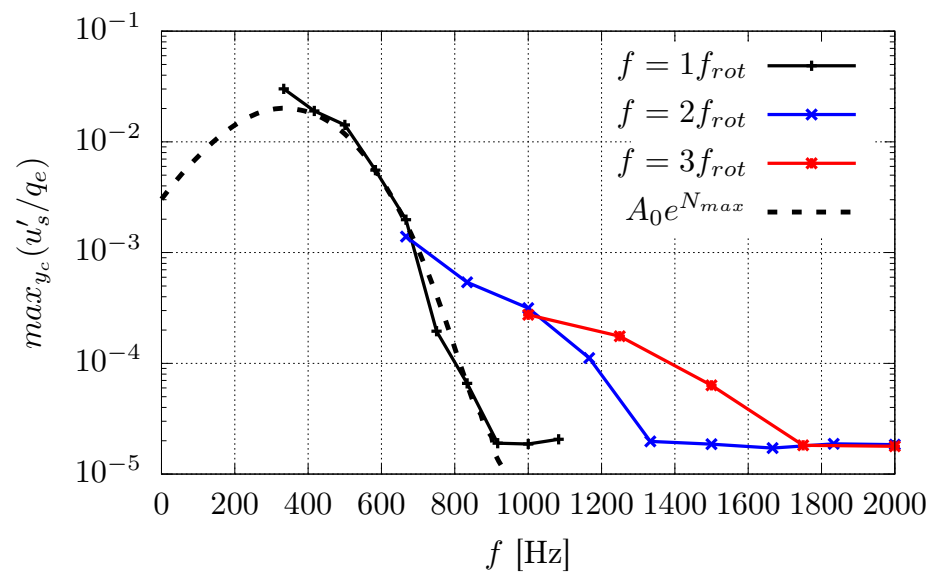

Abbildung 8.1.8: Spannweitig maximale Amplitude instationärer $u_{s}$-Fluktuationen bei ausgewählten Frequenzen stromab vom einzeln jeweils mit $f_{\text {rot }}$ betriebenen Drehteller 9

von ihrer Frequenz für drei ausgewählte Fälle bei unterschiedlicher Rotationsfrequenz $f_{\text {rot }}$ dargestellt. Zum Vergleich ist auch der Fall ohne Aktuierung eingezeichnet. Die drei Fälle bei jeweils konstanter Rotationsfrequenz der Anregung sind mit den Kürzeln $a, b$ und $c$ benannt. Man erkennt nun, dass im Amplitudenspektrum an der Frequenz, welche der jeweiligen Rotationsfrequenz entspricht, ein lokales Maximum auftritt, welches mit der Zahl 1 und dem jeweiligen Fallkürzel markiert ist. Zusätzlich werden lokale Maxima bei den Frequenzen beobachtet, welche den jeweiligen ganzzahligen Vielfachen $n$ der jeweiligen Rotationsfrequenz entsprechen. Diese lokalen Maxima sind jeweils bis zum dritten Vielfachen der Rotationsfrequenz anhand $n$ und dem Fallkürzel markiert. Die festgestellten Höherharmonischen der jeweiligen Grundfrequenz, also der Rotationsfrequenz, könnten dadurch erklärt werden, dass sie am Ort der Anregung zusätzlich zu Störungen mit der Grundfrequenz angeregt werden und durch die Grenzschicht angefacht werden. Eine alternative Erklärung ist, dass sie ein Symptom nichtlinear generierter QSIModen sind. Schon Lerche $[61,62]$ beobachtete die nichtlineare Erzeugung von Höherharmonischen in der Grenzschicht, sofern die Instabilität mit der Grundfrequenz eine ausreichend hohe Amplitude erreichte.

Um der Frage nach der Quelle dieser instationären Störungen nachzugehen, sollen ihre bei $x_{c} / c=0.3$ beobachteten Amplituden mit der maximal erwarteten integralen Anfachung einer Anfangsstörung bei $x_{c} / c=0.1$ gemäß der LST- 
Untersuchung verglichen werden. Aus den Linienmessungen zur Einzelanregung mit Drehteller 9 bei Rotationsfrequenzen von $333 \mathrm{~Hz} \leq f_{\text {rot }} \leq 1083 \mathrm{~Hz}$ wurde jeweils die spannweitig maximal beobachtete Amplitude $u_{s}^{\prime}$ bei $f=f_{\text {rot }}$, $f=2 f_{\text {rot }}$ und $f=3 f_{\text {rot }}$ extrahiert und in Abbildung 8.1 .8 abhängig von $f$ aufgetragen. Ebenfalls eingezeichnet ist die Verteilung der gemäß LSTUntersuchung bei der Messposition $x_{c} / c=0.3$ bzgl. $\beta$ maximal erwarteten Amplituden $u_{s, L S T}^{\prime}(f)=A_{0} \exp \left(N_{\max }(f)\right)$ (bei frequenzunabhängiger und passend gewählter Anfangsamplitude $\left.A_{0}\right)$. $N_{\max }(f)$ entspricht der Verteilung der bzgl. $\beta$ maximalen N-Faktoren laufender QSI an der Messposition gemäß der LST-Untersuchung, siehe Abschnitt 6.2.2. Man erkennt, dass die spannweitig maximal beobachtete Amplitude bei der jeweiligen Grundfrequenz $f_{\text {rot }}$ für $f>=417 \mathrm{~Hz}$ sehr gut der Verteilung maximal erwarteter Amplituden gemäß LST-Untersuchung an dieser Plattentiefenposition entspricht. Dies wäre nicht zu erwarten, wenn die Amplitude $A_{0}$ der ursächlichen instationären Anregung signifikant von der Rotationsfrequenz abhinge. Diese gute Übereinstimmung deutet also darauf hin, dass die Amplitude $A_{0}$ der instationären Anregung bei $f_{\text {rot }}$ nicht signifikant mit der Rotationsfrequenz steigt, bzw. nahezu unabhängig von der Rotationsfrequenz und vom eingebrachten Drehimpuls ist. Wie am Anfang dieses Abschnitts beschrieben, würde eine frequenzunabhängige Anfangsamplitude $A_{0}$ bedeuten, dass eine instationäre Anregung aufgrund einer vertikalen Auslenkung der Drehtelleroberfläche mit $f_{\text {rot }}$ unwahrscheinlich erscheint. Diese Beobachtung lässt sich allerdings stromab anderer Drehteller nur eingeschränkt bestätigen, siehe Abschnitt A.3 im Anhang.

Die Frequenzen der meisten beobachteten Höherharmonischen liegen oberhalb des Frequenzbereichs der bis zu dieser Plattentiefenposition integral angefachten primären QSI-Moden. Stromauf von $x_{c} / c=0.3$ sind zwar in einem beschränkten Profiltiefenbereich Moden mit Frequenzen $f>800 \mathrm{~Hz}$ angefacht, aber bis $x_{c} / c=0.3$ auch wieder gedämpft, sodass bis zur hier betrachteten Messposition keine integrale lineare Anfachung durch die Grenzschicht zu erwarten ist (siehe Abschnitt 6.2.2 und insbesondere Abbildung 6.2.2(a)). Die für $u_{s, L S T}^{\prime}(f)$ in Abbildung 8.1.8 passend angenommene Anfangsamplitude beträgt $A_{0} / q_{e}=6 \cdot 10^{-5}$. Die Höherharmonischen wurden teilweise mit deutlich höheren Amplituden beobachtet als gemäß der Verteilung $u_{s, L S T}^{\prime}(f)$ im jeweiligen Frequenzbereich erwartet werden kann. Wären sie Symptom linearer QSI-Moden mit einer Anregung am Ort der Drehteller, hätten sie dort mit signifikant höherer Amplitude angeregt werden müssen als die für $u_{s, L S T}^{\prime}(f)$ angenommene Anfangsamplitude $A_{0}$. Liegt ihre Frequenz 


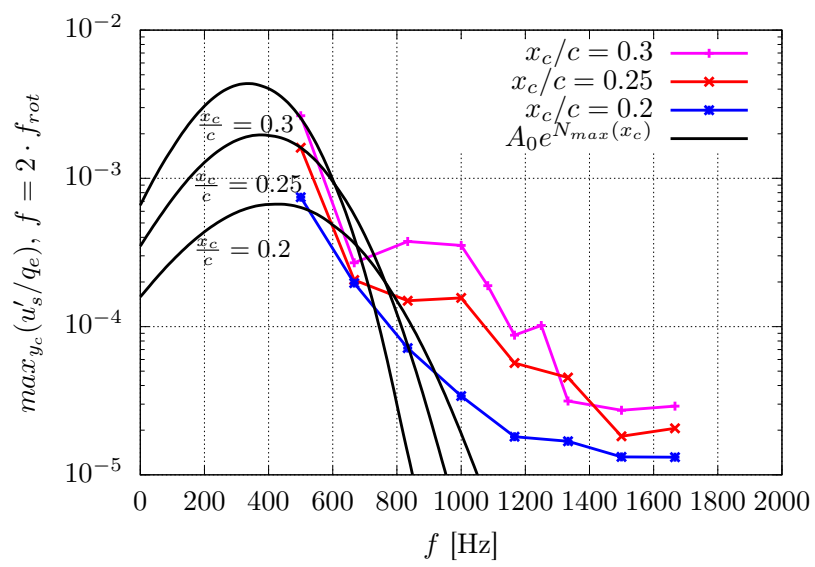

Abbildung 8.1.9: Spannweitig maximale Amplitude $u_{s}^{\prime} / q_{e}$ bei der Frequenz $f=$ $2 \cdot f_{r o t}$ an drei unterschiedlichen Plattentiefenpositionen $x_{c} / c$ stromab von dem mit $f_{\text {rot }}$ betriebenen Drehteller 28

oberhalb der Grenzfrequenz des linear integral angefachten Frequenzbereichs, müsste ihre Amplitude zudem stromab abnehmen.

Daher soll nun die Amplitudenentwicklung von Schwankungen mit $f=2 f_{\text {rot }}$ stromab der Einzelanregung untersucht werden. Dazu wurde die Wirkung der Einzelanregung durch einen ausgewählten Drehteller, Drehteller 28, abhängig von der Rotationsfrequenz $f_{\text {rot }}$ an drei unterschiedlichen Plattentiefenpositionen mithilfe von spannweitigen Linienmessungen in seinem Nachlauf bei konstantem Wandabstand untersucht. In Abbildung 8.1.9 ist dazu jeweils die spannweitig maximale Amplitude instationärer $u_{s}$-Fluktuationen bei $f=2 \cdot f_{\text {rot }}$ für die drei untersuchten Plattentiefenpositionen dargestellt. Mit eingezeichnet ist wieder $u_{s, L S T}^{\prime}(f)$ mit einer konstant und frequenzunabhängig angenommenen Anfangsamplitude $A_{0}$. Für die niedrigste untersuchte Rotationsfrequenz $f_{\text {rot }}=250 \mathrm{~Hz}$ entwickelt sich die Amplitude dieser zweiten Harmonischen mit steigendem $x_{c}$ wie eine primäre laufende Instabilität, da ihre Frequenz $f=2 f_{\text {rot }}=500 \mathrm{~Hz}$ im Frequenzbereich linear angefachter Instabilitäten liegt. Für die Anregung mit $f_{\text {rot }}=333 \mathrm{~Hz}$ wäre gemäß den LSTErgebnissen zu erwarten, dass laufende Instabilitäten mit $f=2 f_{\text {rot }}=666 \mathrm{~Hz}$ zwischen $x_{c} / c=0.2$ und $x_{c} / c=0.25$ noch angefacht, zwischen $x_{c} / c=0.25$ und $x_{c} / c=0.3$ aber bereits gedämpft sind. Die Amplitude, welche bei dieser Frequenz der zweiten Harmonischen beobachtet wurde, nimmt zwar zwischen $x_{c} / c=0.2$ und $x_{c} / c=0.25$ bereits zu, zwischen $x_{c} / c=0.25$ und $x_{c} / c=0.3$ aber stärker als stromauf. Für die höheren untersuchten Rotationsfrequen- 


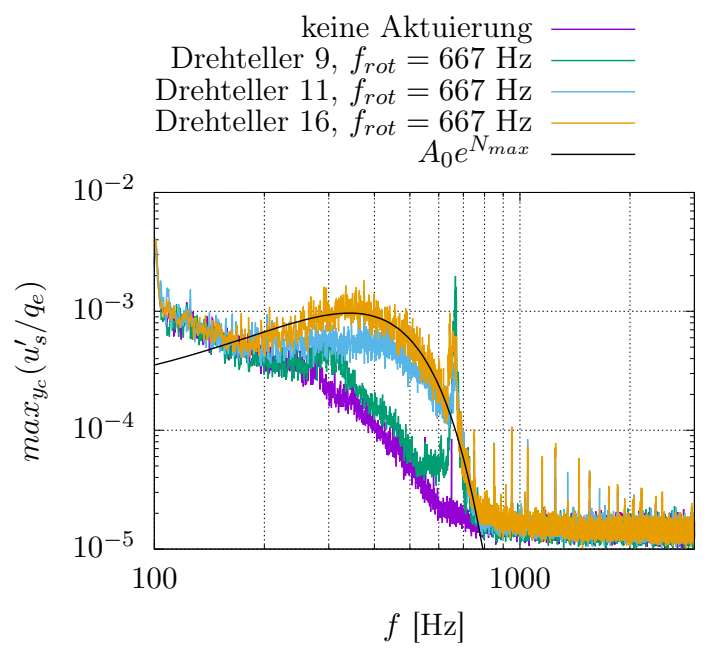

Abbildung 8.1.10: Vergleich des Spektrums spannweitig maximaler instationärer Fluktuationsamplituden im Nachlauf von drei verschiedenen einzeln betriebenen Drehtellern bei einer Rotationsfrequenz von $f_{\text {rot }}=667 \mathrm{~Hz}$

zen wären gemäß LST alle Primärinstabilitäten mit Frequenzen $f=2 f_{\text {rot }}$ zwischen $x_{c} / c=0.2$ und $x_{c} / c=0.3$ gedämpft. Im Experiment wurde allerdings in allen Fällen außer im Fall mit $f_{\text {rot }}=667\left(2 f_{\text {rot }}=1333 \mathrm{~Hz}\right)$ in Plattentiefenrichtung ein monotones Wachstum der Amplitude der zweiten Harmonischen beobachtet. Dies sind Hinweise darauf, dass es sich bei den beobachteten lokalen Amplitudenmaxima bei $2 f_{\text {rot }}$ um nichtlinear angefachte Instabilitäten handelt.

\section{Spektral verteilte Anhebung instationärer Störungen}

Zusätzlich zu den lokalen Maxima bei ganzzahligen Vielfachen von $f_{\text {rot }}$ wurden bei der systematischen Variation der Rotationsfrequenz einzelner Drehteller teilweise erhöhte Amplituden instationärer Fluktuationen in einem breiten Frequenzbereich beobachtet. Dabei wurden ebenfalls deutliche quantitative Unterschiede zwischen einzelnen Drehtellern festgestellt.

In Abbildung 8.1.10 sind die Spektren spannweitig maximaler instationärer Fluktuationsamplituden im Nachlauf von drei verschiedenen einzeln mit derselben Rotationsfrequenz $f_{\text {rot }}=667 \mathrm{~Hz}$ betriebenen Drehtellern dargestellt. Dabei erkennt man, dass bei der Frequenz, welcher der Rotationsfre- 
quenz des Drehtellers entspricht, für alle drei Drehteller ein lokales Maximum mit vergleichbarer Amplitude beobachtet wurde. Im Frequenzbereich $200 \mathrm{~Hz}<f<600 \mathrm{~Hz}$ sind die Amplituden stromab von Drehteller 11 und 16 breitbandig gegenüber dem Fall ohne Aktuierung angehoben. Auch stromab von Drehteller 9 ist eine solche Anhebung zu beobachten, allerdings ist sie deutlich schwächer ausgeprägt.

In Abbildung 8.1.10 ist wie bisher eine theoretische Amplitudenverteilung $u_{s, L S T}^{\prime}$ mit passend gewählter Anfangsamplitude $A_{0}$ eingezeichnet. Im Vergleich erkennt man, dass die angehobenen Amplituden, welche stromab der Drehteller 11 und 16 festgestellt wurden, gut mit der theoretischen Amplitudenverteilung übereinstimmen. Unter der Annahme, dass es sich hierbei um die Auswirkung einer instationären Anregung am Ort des Drehtellers handelt, welche durch die Grenzschicht bis zur Messposition linear angefacht wurde, weist dies darauf hin, dass die Anregung für Drehteller 11 und 16 im Frequenzraum breit verteilt und ihre Anfangsamplitude $A_{0}$ in einem breiten Frequenzbereich konstant ist.

Da eine solche breitbandige Anregung in stark unterschiedlichem Ausmaß stromab der Drehteller 9, 11 und 16 beobachtet wurde, liegt die Annahme nahe, dass es sich um die Konsequenz eines Vorgangs am Drehteller selbst handelt.

\section{Störungen bei Subharmonischen der Rotationsfrequenz}

Im Nachlauf einiger einzeln betriebenen Drehtellern sind im Spektrum spannweitig maximaler instationärer $u_{s}$-Fluktuationsamplituden lokale Maxima bei ganzzahligen Teilern der Rotationsfrequenz zu beobachten, im Folgenden auch als Subharmonische bezeichnet. Dazu sind in Abbildung 8.1.11 wie in Abbildung 8.1.10 die Spektren spannweitig maximaler Amplituden bei $x_{c} / c=0.3 \mathrm{im}$ Nachlauf von drei einzeln bei derselben Rotationsfrequenz $f_{\text {rot }}=833 \mathrm{~Hz}$ betriebenen Drehtellern im Vergleich mit dem Referenzfall ohne Aktuierung dargestellt.

Dabei ist im Vergleich mit $u_{s, L S T}^{\prime}$ bei passend und frequenzunabhängig gewählter Anfangsamplitude $A_{0}$ wieder zu erkennen, dass stromab von Drehteller 16 eine Anhebung der Amplituden in einem breiten Frequenzbereich festgestellt wurde. Diese angehobene Verteilung passt gut zur integralen linearen Anfachung einer im Frequenzspektrum breit verteilten Anregung. Stromab von Drehteller 9 und 11 konzentriert sich die Anhebung jedoch 


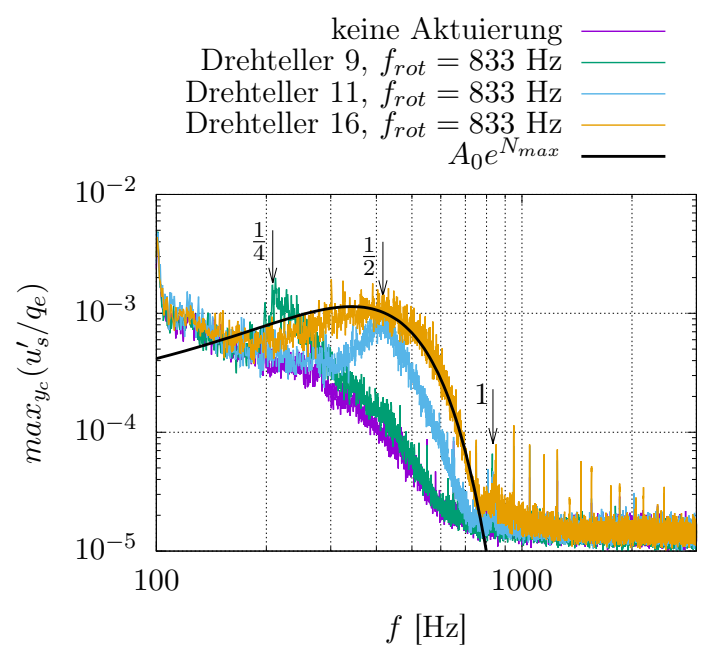

Abbildung 8.1.11: Beobachtete lokale Maxima bei Subharmonischen der Anregungsfrequenz in den spannweitig maximalen Spektren bei $x_{c} / c=0.3$ im Nachlauf hinter drei verschiedenen jeweils einzeln betriebenen Drehtellern bei einer Rotationsfrequenz von $f_{\text {rot }}=833 \mathrm{~Hz}$

um ein lokales Maximum, welches für Drehteller 11 bei $f=\frac{1}{2} f_{\text {rot }}$ und für Drehteller 9 bei $f=\frac{1}{4} f_{\text {rot }}$ liegt. Wie bei der beobachteten Anhebung instationärer Fluktuationen in einem breiten Frequenzbereich hängt es vom jeweils verwendeten Drehteller und von der Rotationsfrequenz ab, ob lokale Maxima bei Subharmonischen festgestellt werden, welche Subharmonischen auftreten und wie stark. Daher scheint es sich auch bei diesem Phänomen um eine Konsequenz eines Vorgangs am Drehteller bzw. am Ort der Anregung direkt zu handeln. Um einen Überblick darüber zu gewähren, dass die verschiedenen vorgestellten Phänomene angehobener instationärer Störungen stromab von verschiedenen Drehtellern in unterschiedlichem Ausmaß und bei unterschiedlichen Rotationsfrequenzen beobachtet werden, werden im Anhang die Spektren instationärer Fluktuationen stromab von Drehteller 9,11,16 und 28 im gesamten untersuchten Bereich von $f_{\text {rot }}$ als Isokonturdiagramme dargestellt (Abschnitt A.4).

\section{Drehteller ohne signifikante Anhebung instationärer Störungen}

Um den Unterschied zwischen den Drehtellern weiter zu untersuchen, wurde eine Drehzahl ausgewählt, welche $q_{B} / q_{e}=0.23$ bzw. $f_{\text {rot }}=833 \mathrm{~Hz}$ entspricht. 
(a)

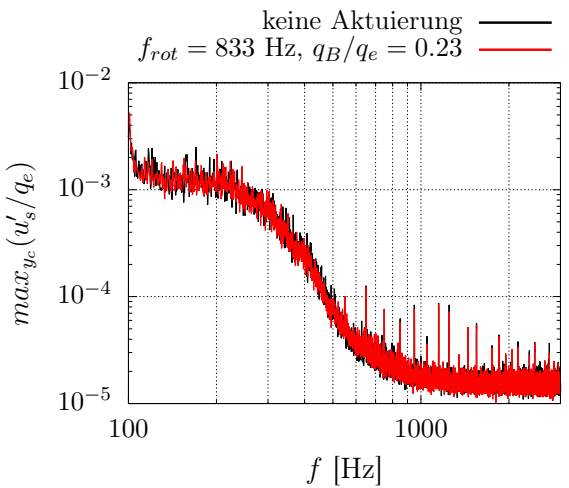

(b)

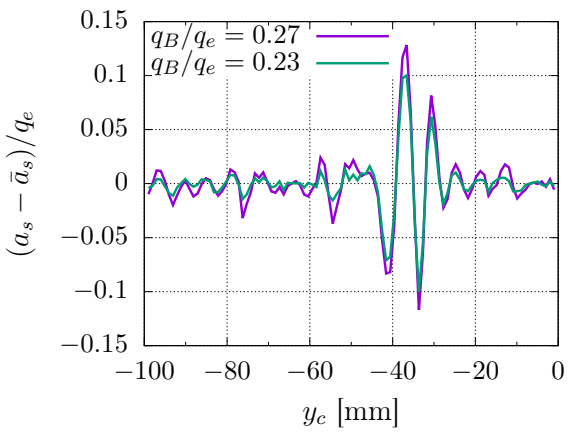

Abbildung 8.1.12: (a) Spektrum spannweitig maximaler instationärer Fluktuationsamplituden stromab von Drehteller 20 , der mit $f_{\text {rot }}=833 \mathrm{~Hz}$ bzw. $q_{B} / q_{e}=0.23$ betrieben wurde, im Vergleich zum Fall ohne Aktuierung (beides gemessen bei $x_{c} / c=0.3$ ) (b) Die isolierte eingebrachte stationäre Grenzschichtdeformation in diesem und einem weiteren Fall

Für 20 jeweils einzeln mit dieser Rotationsfrequenz betriebene Drehteller wurde in ihrem Nachlauf eine Linienmessung durchgeführt. Dabei fielen zwei Drehteller (20 und 22) auf, die bei dieser Rotationsfrequenz keine signifikante Veränderung des instationären Spektrums in ihrem Nachlauf hervorriefen. Das Spektrum spannweitig maximaler instationärer Fluktuationsamplituden bei $x_{c} / c=0.3 \mathrm{im}$ Nachlauf von Drehteller 20 ist in Abbildung 8.1.12(a) im Vergleich zum nicht-aktuierten Zustand dargestellt. Es sind keine signifikanten Unterschiede und keines der in den vorigen Teilabschnitten diskutierten Phänomene zu erkennen. Die gleiche Beobachtung gilt für Drehteller 22, für den die äquivalente Darstellung in Abb. A.5.1(a) im Anhang gezeigt wird. Stromab anderer Drehteller wurde bei derselben Rotationsfrequenz eine signifikante Anhebung instationärer Fluktuationsamplituden beobachtet, siehe Abbildung 8.1.11. Auch bei einer höheren Rotationsfrequenz $\left(f_{\text {rot }}=\right.$ $1000 \mathrm{~Hz}$ ) werden stromab von Drehteller 20 keine signifikant angehobenen instationären Fluktuationen beobachtet (siehe Abb. A.5.2 im Anhang).

Gleichzeitig bringen die Drehteller 20 und 22 aber eine signifikante stationäre Deformation in die Grenzschicht ein, wie in Abbildung 8.1.12(b) für Drehteller 20 in Form der isolierten stationären Aktuierungswirkung $a_{s}\left(y_{c}\right)$ bei abgezogenem spannweitigen Mittelwert $\bar{a}_{s}$ dargestellt ist (äquivalente Darstellung für Drehteller 22 in Abb. A.5.1(b) im Anhang). In Kombination 
mit der Beobachtung, dass die instationären Phänomene bei unterschiedlichen Drehtellern in unterschiedlichem Ausmaß und in verschiedenen Drehzahlbereichen auftreten, legen die Ergebnisse für Drehteller 20 und 22 also nahe, dass es sich bei einem Großteil der zusätzlich angehobenen instationären Fluktuationen um Phänomene handelt, welche hauptsächlich der technischen Realisierung zuzuordnen und keine inhärente Eigenschaft der Rotationsanregung sind. In der Grenzschicht stromab von einer idealisierten rotierenden Oberfläche wären sie daher nicht zu erwarten. Insbesondere die zu Beginn dieses Abschnitts geschilderte mögliche Ursache für angehobene instationäre Fluktuationen, eine zusätzliche Instabilität durch starke Geschwindigkeitsgradienten am Drehtellerrand, kann zumindest bei den mit Drehteller 20 untersuchten Rotationsfrequenzen $f_{\text {rot }}=833 \mathrm{~Hz}$ und $f_{\text {rot }}=1000 \mathrm{~Hz}$ keine signifikante Rolle spielen. Da es sich hierbei um besonders hohe Rotationsfrequenzen handelt und eine signifikante stationäre Störung eingebracht wurde, sind besonders starke Gradienten am Drehtellerrand zu erwarten. Es ist also wahrscheinlich, dass dieser Erklärungsansatz auch bei geringeren Werten von $f_{\text {rot }}$ keine signifikante Rolle spielt.

\subsection{Transitionsszenario im Fall mit Rotationsaktuierung}

Im Folgenden soll untersucht werden, welchen Einfluss die Anregung der stationären Kontrollmode durch verteilte Rotationsaktuierung auf die gesamte Grenzschichtströmung stromab der Anregung hat. Insbesondere soll natürlich geklärt werden, ob sie zu einer Transitionsverzögerung im Sinne der UFD-Methode führt. Dafür wurden die Drehteller 9 bis 29 mit einer Rotationsfrequenz betrieben, für die bei $x_{c} / c=0.3$ eine signifikante Anhebung der stationären Kontrollmode mit der spannweitigen Wellenlänge von $\lambda=6 \mathrm{~mm}$ beobachtet wurde $\left(\hat{u}_{s} / q_{e}=0.065\right.$, siehe Abb. 8.1.1(a)). Die Drehteller wurden in der Aufsicht auf die Modellplatte gegen den Uhrzeigersinn (GUZ) betrieben und die gewählten Betriebsparameter entsprachen (vgl. Abschnitt 8.1.1):

$$
\begin{aligned}
q_{B} / q_{e} & =0.205 \\
f_{\text {rot }} & =750 \mathrm{~Hz}
\end{aligned}
$$

Damit lag die Rotationsfrequenz oberhalb des Frequenzbereichs der bis zum Plattenende stärksten integralen Anfachung laufender QSI. Die Amplitude der angeregten Kontrollmode hatte bei $x_{c} / c=0.3$ einen ähnlichen Wert wie bei den Experimenten mit einem pneumatischen Aktuator im selben 


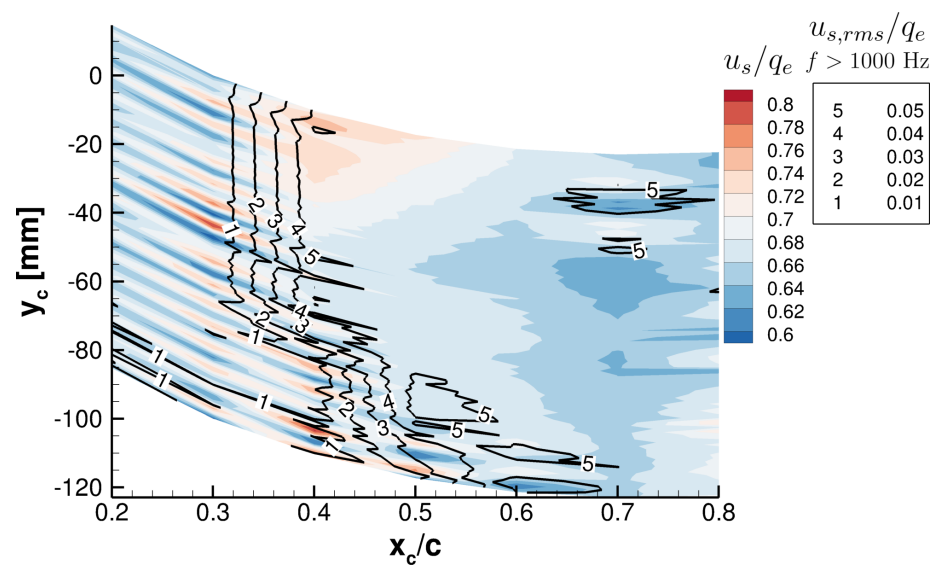

Abbildung 8.2.1: Transitionsszenario stromab einer verteilten Aktuierung durch rotierende Drehteller bei einer Rotationsfrequenz von $f_{\text {rot }}=750 \mathrm{~Hz}\left(q_{B} / q_{e}=0.205\right)$

experimentellen Aufbau, bei denen eine Transitionsverzögerung gemäß der UFD-Methode nachgewiesen werden konnte [68].

Im Nachlauf stromab der gemeinsam betriebenen Drehteller wurden Linienmessungen zwischen $0.2<x_{c} / c<0.8$ in $x_{c}$-Schritten von $\Delta x_{c} / c=0.1$ über einer spannweitigen Strecke von $\Delta y_{c}=99.99 \mathrm{~mm}$ bei jeweils konstantem Wandabstand in der Grenzschicht durchgeführt. Der Wandabstand wurde dabei je Profiltiefenposition so gewählt, dass die zeitlich und spannweitig gemittelte Geschwindigkeit bei $\frac{u_{s}}{q_{e}} \approx 0.7$ lag. Dieser Abstand wurde als ein Kompromiss zwischen dem Wandabstand des Amplitudenmaximums der beteiligten stationären QSI und instationärer Phänomene gewählt. Die in Abschnitt 8.1.1 beschriebenen Einschränkungen bzgl. der Zuverlässigkeit der Motoren machten dreidimensional verteilte Messungen in der Grenzschicht aufgrund ihrer zeitlichen Anforderungen unmöglich.

Als Überblick über das vermessene Grenzschichtströmungsfeld ist in Abbildung 8.2.1 die gemessene zeitlich gemittelte $u_{s}$-Geschwindigkeitskomponente in farblichen Isokonturen abhängig von der spannweitigen Koordinate $y_{c}$ und der Plattentiefenkoordinate $x_{c}$ dargestellt. Die Isolinien markieren den Effektivwert instationärer $u_{s}$-Fluktuationen mit $f>1000 \mathrm{~Hz}$. In Abschnitt 6.3 wurden sie mit Sekundärinstabilitäten oder turbulenten Fluktuationen assoziiert. Bei der Darstellung ist zu beachten, dass Messdaten nur in Plattentiefenschritten von $\Delta x_{c} / c=0.1$ vorliegen und für die Darstellung zwischen den Stützstellen linear interpoliert wurden. 


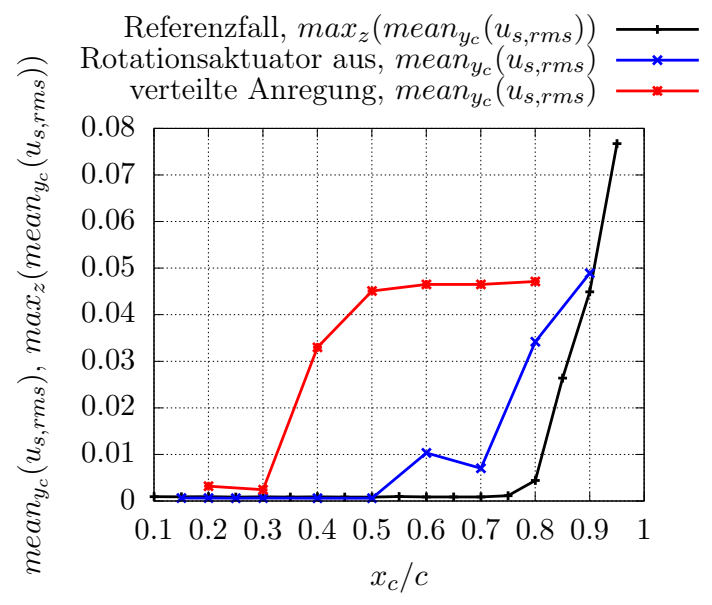

Abbildung 8.2.2: Spannweitig gemittelter Effektivwert instationärer $u_{s}$-Fluktuationen mit $f>1000 \mathrm{~Hz}$ im Vergleich stromab von einer verteilten Rotationsaktuierung mit $f_{\text {rot }}=750 \mathrm{~Hz}\left(q_{B} / q_{e}=0.205\right)$, stromab von still stehenden Drehtellern und im Referenzfall ohne Drehteller (siehe Abschnitt 6.3)

Bei $x_{c} / c=0.2$ und $x_{c} / c=0.3$ ist deutlich die spannweitige Periodizität mit der Wellenlänge $\lambda=6 \mathrm{~mm}$ zu erkennen. Diese gehört zu der erfolgreich angeregten stationären Kontrollmode. Stromab von $x_{c} / c=0.3$ ist ein unterschiedliches Verhalten für $y_{c}<-65 \mathrm{~mm}$ und für $y_{c}>-65 \mathrm{~mm}$ bzw. den jeweils stromab verfolgten spannweitigen Bereich zu beobachten. Für $y_{c}>-65 \mathrm{~mm}$ wachsen die Effektivwerte hochfrequenter instationärer Fluktuationen zwischen $x_{c} / c=0.3$ und $x_{c} / c=0.4$ rasch an und die spannweitige Periodizität der stationären Grenzschichtdeformation verschwindet. Dies deutet darauf hin, dass die stationären Längswirbel zerfallen. Im Bereich $y_{c}<-60 \mathrm{~mm}$ folgen diese Beobachtungen erst stromab von $x_{c} / c=0.4$. Das Hochschnellen der Effektivwerte hochfrequenter Fluktuationen und die zerfallenden stationären Längswirbel deuten auf den laminar-turbulenten Umschlag hin, sodass die Effektivwerte stromab von dieser Plattentiefenposition hauptsächlich turbulente Fluktuationen repräsentieren.

Zur Verdeutlichung ist in Abbildung 8.2.2 der spannweitig gemittelte Effektivwert hochfrequenter Fluktuationen im Verlauf entlang $x_{c}$ dargestellt. Zum Vergleich ist er auch für den Fall mit eingebautem Rotationsaktuator, aber ausgeschalteten Motoren, ebenfalls aus Linienmessungen bei konstantem Wandabstand, dargestellt. Weiterhin ist zum Vergleich der Verlauf des spannweitig gemittelten, aber wandnormal maximalen Effektivwertes hoch- 
frequenter Störungen für den in Abschnitt 6.3 beschriebenen Referenzfall dargestellt, da für diesen dreidimensional verteilte Messungen durchgeführt wurden. Es ist zu beachten, dass für die Messungen im Nachlauf des Rotationsaktuators nicht die wandnormal maximalen Effektivwerte beobachtet werden konnten, da die hochfrequenten Sekundärinstabilitäten typischerweise ihren Ursprung näher an der Wand haben als der konstant verwendete Wandabstand. Da sich diese hochfrequenten Störungen aber während des Umschlags schnell innerhalb der Grenzschicht ausbreiten, ist der maximale Gradient des spannweitig gemittelten Effektivwerts in Profiltiefenrichtung trotzdem ein guter Indikator für die spannweitig gemittelte Transitionslage.

Man erkennt, dass im Vergleich zum Referenzfall ohne Drehteller die Transitionslage stromab des Rotationsaktuators bereits im ausgeschalteten Zustand etwas stromauf verschoben zu sein scheint. Statt wie im Referenzfall zwischen $x_{c} / c=0.8$ und $x_{c} / c=0.9$ ist im Fall des ausgeschalteten Rotationsaktuators der steilste Gradient des Effektivwerts hochfrequenter Fluktuationen in Profiltiefenrichtung zwischen $x_{c} / c=0.7$ und $x_{c} / c=0.8$ zu beobachten. Maßgeblicher Unterschied zwischen den verwendeten Naseneinsätzen der beiden Fälle sind die zur Grenzschichtströmung exponierten Drehteller, welche auch im ausgeschalteten Zustand eine stationäre Störanregung darstellen. Die spannweitige Periodizität dieser Anregung hat konstruktionsbedingt eine Wellenlänge von $\lambda=6 \mathrm{~mm}$. Die allein dadurch angeregte stationäre Kontrollmode erreicht aber keine ausreichend große Amplitude, um eine nichtlineare Vordeformation der Grenzschicht gemäß der UFD-Methode zu erreichen, sondern scheint durch das angehobene Störungsniveau zu einer Verschiebung der Transitionslage stromauf zu führen.

Im aktuierten Fall wird zwar bei $x_{c} / c=0.3$ eine erfahrungsgemäß (siehe [68]) ausreichend große Amplitude der stationären QSI mit $\lambda=6 \mathrm{~mm}$ beobachtet, um die gewünschte Vordeformation zu erzeugen, gleichzeitig werden aber auch die Amplituden laufender QSI stark angehoben. In Abbildung 8.2.3 sind für die drei am weitesten stromauf gemessenen spannweitigen Linienmessungen im Nachlauf der beschriebenen verteilten Aktuierung die Spektren spannweitig gemittelter instationärer Geschwindigkeitsfluktuationen dargestellt. Zum Vergleich ist auch das Spektrum bei $x_{c} / c=0.2$ ohne Aktuierung dargestellt. Weiterhin sind für $x_{c} / c=0.2$ und $x_{c} / c=0.3$ die Verteilungen der bzgl. $\beta$ maximalen integralen Anfachung $u_{s, L S T}^{\prime}(f)$ einer passend gewählten frequenzunabhängigen Anfangsamplitude $A_{0}$ dargestellt. Diese Anfangsamplitude wurde so gewählt, dass $u_{s, L S T}^{\prime}(f)$ zwischen $500 \mathrm{~Hz}<f<700 \mathrm{~Hz}$ etwa der gemessenen Amplitudenverteilung bei $x_{c} / c=0.2$ entspricht. Man erkennt, 


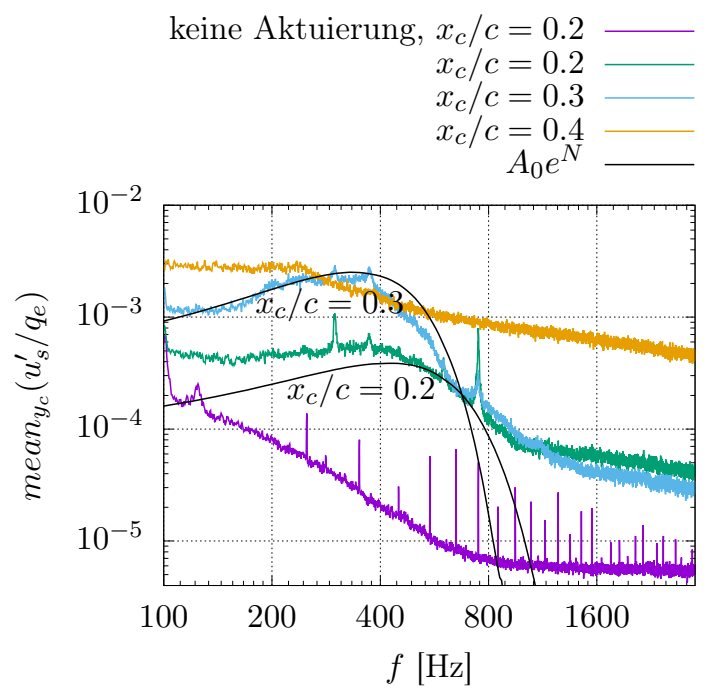

Abbildung 8.2.3: Spektren der spannweitig gemittelten instationären Fluktuationsamplituden stromab der verteilten Anregung bei den Profiltiefenpositionen $x_{c} / c=0.2,0.3$ und 0.4

dass durch die verteilte Aktuierung schon bei $x_{c} / c=0.2$ die Amplituden instationärer $u_{s}$-Fluktuationen signifikant angehoben sind. Ein ausgeprägtes lokales Maximum wird bei der Rotationsfrequenz $f_{\text {rot }}=750 \mathrm{~Hz}$ beobachtet, dessen Amplitude allerdings zwischen $x_{c} / c=0.2$ und $x_{c} / c=0.3$ abnimmt. Wie in Abschnitt 6.2.2 diskutiert wurde (vgl. Abbildung 6.2.2(a)), sind laufende QSI mit einer Frequenz von $750 \mathrm{~Hz}$ nur im vorderen Plattentiefenbereich integral angefacht und die beobachtete Dämpfung ihrer Amplitude entspricht der Erwartung gemäß den LST-Ergebnissen. Der bzgl. $\beta$ maximale N-Faktor bei $f=750 \mathrm{~Hz}$ nimmt zwischen $x_{c} / c=0.2$ und $x_{c} / c=0.3 \mathrm{um}$ $\Delta N=-0.696 \mathrm{ab}$. Dies entspricht einem erwarteten Amplitudenverhältnis zwischen $x_{c} / c=0.2$ und $x_{c} / c=0.3$ von $\frac{u_{s}^{\prime}\left(x_{c} / c=0.2\right)}{u_{s}^{\prime}\left(x_{c} / c=0.3\right)}=\exp (\Delta N)=0.5$, was näherungsweise der beobachteten Abnahme der Amplitude bei $f_{\text {rot }}$ entspricht.

Die Tatsache, dass die Amplitude laufender QSI, deren Frequenz der Rotationsfrequenz entspricht, bis $x_{c} / c=0.3$ abnimmt, deutet darauf hin, dass sie nicht maßgeblich für den verfrühten laminar-turbulenten Umschlag verantwortlich sind. Man erkennt in den Spektren aber auch, dass die verteilte Aktuierung eine signifikante und breitbandige Anhebung der Amplituden von $u_{s}$-Fluktuationen bewirkt. Diese Anhebung führt besonders im Frequenz- 
bereich linear stark angefachter QSI zwischen $100 \mathrm{~Hz}<f<600 \mathrm{~Hz} \mathrm{~Hz}$ bis $x_{c} / c=0.3$ zu signifikanten Amplituden. $\mathrm{Ab} x_{c} / c=0.4$ ähneln die spannweitig gemittelten Spektren bereits einem turbulenten Spektrum (siehe Abschnitt 6.3). Gemeinsam mit der in Abbildung 8.2.1 beobachteten spannweitig weitestgehend homogenen Transitionsfront weist dies darauf hin, dass es sich hierbei um eine durch laufende QSI verursachte Transition handelt (siehe Abschnitt 2.3 bzw. [97]). Die Annahme liegt nahe, dass bei verteilter Anregung mit vielen Drehtellern gleichzeitig die bereits stromab von einzelnen Drehtellern beobachteten instationären Fluktuationen (siehe Abschnitt 8.1.3) sich überlagern und zu einer spannweitig verteilten Anhebung instationärer Fluktuationen führen. Dabei scheint die bzgl. der Frequenz breit verteilte Anhebung besonders relevant zu sein. Die Drehteller, deren Nachlauf bei $x_{c} / c=0.3$ bei $y_{c}<-65 \mathrm{~mm}$ beobachtet wird, heben solche Fluktuationen offenbar mit etwas niedrigerer Amplitude an als diejenigen, deren Nachlauf bei $y_{c}>-65 \mathrm{~mm}$ beobachtet wird.

Zusammenfassend lässt sich schlussfolgern, dass in der konkret untersuchten technischen Realisierung die unbeabsichtigt zusätzlich angehobenen laufenden QSI eine erfolgreiche Transitionsverzögerung mit der UFD-Methode verhindern. Stattdessen führt die Rotationsaktuierung zu einem weit stromauf verschobenen finalen Umschlag. Da die Kontrollmode aber mit ausreichend großer Amplitude angeregt wurde und die angehobenen laufenden QSI mit großer Wahrscheinlichkeit keine inhärente Eigenschaft der Rotationsanregung darstellen (siehe Abschnitt 8.1.3), bleibt eine Transitionsverzögerung bei verbesserter technischer Realisierung aber prinzipiell möglich. 


\section{Kapitel 9}

\section{Zusammenfassung und Ausblick}

\subsection{Zusammenfassung}

Im Rahmen dieser Arbeit wurde zur Untersuchung und Beeinflussung querströmungsdominierter Transition in einer dreidimensionalen Grenzschicht eine neue experimentelle Konfiguration ausgelegt, aufgebaut und etabliert. Der spezielle Fokus lag auf einer Methode zur Transitionsverzögerung namens Upstream Flow Deformation (UFD), in der zusätzliche Querströmungsinstabilitäten (QSI) künstlich angeregt werden und durch nichtlineare Wechselwirkungen eine stabilisierende Vordeformation der Grenzschicht erreicht wird. Es wurden zwei Konzepte für eine solche künstliche Anregung untersucht. Dabei konnte nachgewiesen werden, dass sowohl durch spannweitig periodische lokale Heizung der Modelloberfläche als auch durch eine spannweitige Reihe von rotierenden oberflächenbündig integrierten Scheiben eine stationäre QSI-Kontrollmode angeregt wurde. Für beide Konzepte wurde ein eindeutiger Zusammenhang der Kontrollmoden-Amplitude mit der jeweiligen Anregungsamplitude festgestellt. Des Weiteren wurde geprüft, wie gut es jeweils gelang, die stationäre Kontrollmode isoliert von anderen Störungen anzuregen. Bei der Heizaktuierung wurde für bestimmte Fälle auch eine Reduktion der eigentlich anzuregenden QSI-Amplitude festgestellt, was als destruktive Überlagerung der Heizanregung und der Anregung durch die Rau- 
igkeitseigenschaft der Heizelemente selbst interpretiert wurde. Des Weiteren wurde der Einfluss der Aktuierungsposition und der Heizelementlänge auf die Effizienz der Störungseinbringung untersucht. Bei der Rotationsaktuierung wurde diese Effizienz abhängig vom Drehsinn der Drehteller verglichen. Zusätzlich wurde bereits mit einem einzeln betriebenen Drehteller eine Deformation der zeitlich gemittelten Grenzschichtströmung nachgewiesen und ihre Amplitude abhängig von der Rotationsfrequenz quantifiziert. Jeweils für einen ausgewählten Satz von Betriebsparametern wurde die Entwicklung der Grenzschichtströmung stromab der künstlichen Anregung untersucht. Eine Transitionsverzögerung wurde nicht erreicht. Die Gründe dafür konnten aber durch detaillierte Messungen überzeugend nachvollzogen werden. Dies liefert wertvolle Ansätze für zukünftige Experimente zur UFD-Methode.

Im Folgenden sollen die wesentlichen Ergebnisse detaillierter zusammengefasst werden.

\section{Das neu ausgelegte Prinzipexperiment}

Die neue experimentelle Konfiguration basiert auf dem ursprünglichen Prinzipexperiment der schiebenden ebenen Platte (siehe [8, 11, 79]). Auch die neu ausgelegte Konfiguration besteht im Wesentlichen aus einer Modellplatte und einem Verdrängungskörper, der eine geeignete Druckverteilung auf die Modellplatte aufprägt. Ziel der Neuauslegung waren vor allem geeignete Stabilitätseigenschaften der dreidimensionalen Grenzschicht an der Modellplatte für die Untersuchung von Aktuierungskonzepten im Rahmen der UFD-Methode. Für die im Rahmen dieser Arbeit durchgeführten Untersuchungen wurde das neue Prinzipexperiment im $1 \mathrm{MG}$ in Göttingen eingesetzt. Als Hauptmessmethode wurde die Hitzdrahtanemometrie verwendet, um die stationären und instationären Grenzschichtprozesse quantitativ zu charakterisieren.

Die realisierte Grenzschichtströmung im neu ausgelegten Prinzipexperiment wurde zunächst bzgl. der Grund- und Störströmung untersucht und mit numerischen Untersuchungen verglichen. Dabei konnte gezeigt werden, dass die Grenzschicht die Ziele der Neuauslegung voll erfüllt. Lohse et al. konnten mithilfe eines pneumatischen Aktuators bereits experimentell nachweisen, dass eine Transitionsverzögerung mithilfe der UFD-Methode im neu ausgelegten Prinzipexperiment möglich ist [68, 70]. 


\section{Spannweitig periodische Wandheizung}

Aufgrund der Fragestellung nach der Anwendbarkeit der spannweitig periodischen Wandheizung zur Transitionsverzögerung im Rahmen der UFDMethode wurde dieses Anregungskonzept im Rahmen dieser Arbeit in deutlich größerer Tiefe untersucht als bisher. Die spannweitig periodische Wandheizung wurde elektrisch durch eine in Spannweitenrichtung angeordnete Reihenschaltung von Leiterbahnen mit abwechselnd hohem und niedrigem elektrischen Widerstand erreicht. Die Leiterbahnen mit hohem Widerstand, die sog. Heizelemente, hatten dabei einen spannweitigen Abstand der Kontrollmodenwellenlänge $\lambda=6 \mathrm{~mm}$ zueinander. An verschiedenen Profiltiefenpositionen wurden acht solcher Reihenschaltungen mit zwei verschiedenen Längen der Heizelemente vorgesehen. Es konnte gezeigt werden, dass die Heizaktuierung in der Lage ist, die stationäre Kontrollmode mit wohldefinierter spannweitiger Wellenlänge $\lambda=6 \mathrm{~mm}$ und mit signifikanter Amplitude anzuregen, sowie die Amplitude durch Variation der Heizleistung zu steuern. Mit langen Heizelementen wurde dabei bei ähnlicher Anregungsposition eine effizientere Amplitudensteigerung erreicht als mit kurzen Heizelementen. Bei der Heizleistungsvariation wurde keine systematische direkte Anhebung instationärer Geschwindigkeitsfluktuationen beobachtet, welche über die zu erwartende geringe spannweitig gemittelte thermische Destabilisierung laufender Moden in der Grenzschicht hinausgeht. Dies weist auch darauf hin, dass die Heizaktuierung die Rezeptivität bzgl. instationärer Störungen, welche für laufende QSI relevant sind, nicht systematisch erhöht. Bei geringer Heizleistung wurde der Effekt einer abnehmenden Amplitude der Kontrollmode beobachtet, bevor sie mit weiter zunehmender Heizleistung monoton angehoben werden konnte. Dies konnte als eine destruktive Superposition zweier Anregungsmechanismen interpretiert werden: der Anregung durch Oberflächenrauigkeiten und der Anregung durch die Heizung.

Bei der Untersuchung der Wirkung der Heizaktuierung auf die Entwicklung der Grenzschicht wurde festgestellt, dass im aktuierten Fall in einem gewissen beschränkten Plattentiefenbereich und besonders an ausgewählten spannweitigen und wandnormalen Positionen angehobene instationäre Geschwindigkeitsfluktuationen im Frequenzbereich der gemäß LST-Analyse zu erwartenden laufenden QSI auftreten. Es konnte gezeigt werden, dass es sich dabei um Sekundärinstabilitäten vom Typ III handelt. Diese entstehen durch die starke Deformation der Grenzschicht durch die künstlich angeregte stationäre Kontrollmode und sind daher nicht charakteristisch für die Anregung durch Oberflächenheizung. Durch die Aktuierung wurde an 
vergleichbarer Profiltiefenposition eine ähnliche Amplitude der Kontrollmode erzeugt wie in Fällen erfolgreicher UFD-Transitionsverzögerung (siehe z.B. [68]). Trotzdem führte die Heizaktuierung in der aktuellen Arbeit nicht zu einer Transitionsverzögerung, da durch die Dominanz langwelliger QSI schon im ausgeschalteten Zustand die Aktuierung zusätzlich zur beabsichtigten stabilisierenden Vordeformation zu einer ungünstigen nichtlinearen Wechselwirkung führte. Die Amplitude der zuvor dominanten langwelligen QSIMode wurde erfolgreich reduziert. Parallel dazu wurde aber die Amplitude einer Mode im Bereich spannweitiger Wellenzahlen zwischen der kurzwelligen Kontrollmode und der langwelligen dominanten Mode angehoben, sodass die Aktuierung im Wesentlichen zu einer spektralen Umverteilung stationärer QSI führte. Im Gegensatz zu vielen anderen solcher Experimente in der Literatur, in denen die Anwendung der UFD-Methode fehl schlug (z.B. die Experimente zu [98]), konnte hier durch die detaillierte Charakterisierung des aktuierten und des nicht-aktuierten Falls gezeigt werden, warum es zu keiner Transitionsverzögerung kam. In vielen der bisherigen Experimente zur UFD-Methode wurde zudem die natürlich dominante stationäre QSI-Mode durch diskrete Rauigkeitselemente (DRE) im Referenzfall in ihrer Amplitude und Wellenlänge fixiert, so auch in den Untersuchungen von Lohse et al. [68, 70]. Dadurch wurde eine spannweitig homogene Ausgangssituation des stationären Störungsspektrums geschaffen, welches zudem genau zur ausgewählten Kontrollmode passte. Da dies keine realistische Ausgangssituation darstellt, wurde im Rahmen dieser Arbeit auf eine künstliche Fixierung der natürlich dominanten stationären QSI-Mode durch DRE verzichtet. Somit konnte auch die Empfindlichkeit der UFD-Methode bzgl. der Anfangsbedingungen demonstriert und analysiert werden.

\section{Spannweitig periodische Rotation}

Die Anregung stationärer QSI durch spannweitig periodisch angeordnete rotierende Scheiben wurde zum ersten Mal untersucht. Das Anregungskonzept ist durch eine Rezeptivitätshypothese motiviert, nach der eine besonders effiziente Anregung stationärer QSI zu erwarten wäre. Es wurden 50 Drehteller oberflächenbündig in einer spannweitigen Reihe mit dem Abstand der Kontrollmodenwellenlänge $\lambda=6 \mathrm{~mm}$ zueinander in die Modelloberfläche eingelassen. Die zugehörigen und darunter im Modellinneren fixierten Motoren konnten individuell in ihrer Rotationsfrequenz geregelt werden. Daher konnte eine Anregung sowohl durch spannweitig periodische Rotation als auch durch spannweitig lokalisierte Rotation untersucht werden. Schon mit 
einem einzelnen rotierenden Drehteller war eine signifikante lokale Deformation des zeitlich gemittelten Strömungsfeldes möglich. Die Amplitude $\hat{a}_{s}^{*} / q_{e}$ dieser Deformation folgte einem linearen Zusammenhang mit der Anregungsamplitude. Bei spannweitig periodischer Anregung konnte gezeigt werden, dass die stationäre QSI-Kontrollmode erfolgreich angeregt wurde und die Kontrollmoden-Amplitude durch Variation der Rotationsfrequenz bzw. der Anregungsamplitude $q_{B} / q_{e}$ gesteuert werden kann. Dabei wurde ein linearer Zusammenhang zwischen der Anregungsamplitude und der Amplitude der Kontrollmode festgestellt. Ähnlich zur Heizanregung wurde bei der Rotationsanregung eine spannweitige Phasenverschiebung der QSI-Kontrollmode beobachtet, welche als charakteristische Phasendifferenz zwischen der Anregung durch die Rauigkeitseigenschaft des Drehtellers und der Anregung durch die Rotation interpretiert wurde. Diese Phasendifferenz entsprach etwa dem Durchmesser der Drehteller, weshalb vermutet wird, dass die Zentren der angeregten Längswirbel durch die Rauigkeit und durch die Rotation an spannweitig gegenüberliegenden Rändern des Drehtellers zu verorten sind. Es wurde auch der Einfluss des Drehsinns der Drehteller auf die Rotationsanregung der Kontrollmode untersucht. Ein Drehsinn entgegen des Uhrzeigersinns bei Aufsicht auf die Drehteller entspricht bei dem gewählten Vorzeichen des Anströmungsschiebewinkels dem Drehsinn der stationären Längswirbel im Rahmen der in Abschnitt 2.5.3 aufgestellten Anregungshypothese. Es wurde festgestellt, dass die Rotationsanregung entgegen des Uhrzeigersinns etwas effektiver ist als die Rotationsanregung im Uhrzeigersinn. Allerdings ändert sich die beobachtete charakteristische Phasendifferenz zur Rauigkeitsanregung durch den Drehteller kaum bei Umkehr des Drehsinns, während im Rahmen der Hypothese eine Änderung um $\lambda / 2$ zu erwarten gewesen wäre. Die Beobachtungen unterstützen also zumindest im untersuchten Drehzahlbereich die vorgestellte Anregungshypothese nicht.

Die Wirkung der spannweitig periodischen Anregung bei gleicher und zeitlich konstanter Rotationsfrequenz auf die Grenzschicht stromab wurde an mehreren Plattentiefenpositionen für eine ausgewählte Rotationsfrequenz untersucht. Die Rotationsfrequenz wurde dabei so gewählt, dass eine ähnliche Amplitude der stationären Kontrollmode eingebracht wurde wie bei bisherigen erfolgreichen UFD-Anwendungen zur Transitionsverzögerung. Es wurden aber auch signifikant angehobene Amplituden laufender QSI beobachtet. Der finale Umschlag wurde durch die Aktuierung deutlich stromauf verlagert und wies klare Eigenschaften einer Dominanz laufender QSI auf. Schon stromab der spannweitig lokalisierten Einzelanregung wurden abhängig vom verwendeten Drehteller und der Rotationsfrequenz unterschiedliche 
instationäre Phänomene beobachtet. Durch den Vergleich der beobachteten instationären Phänomene stromab von unterschiedlichen einzeln rotierenden Drehtellern wurde gezeigt, dass die Phänomene jeweils in unterschiedlichem Ausmaß und bei unterschiedlicher Rotationsfrequenz auftreten. Zusätzlich wurde stromab von zwei einzeln betriebenen Drehtellern keine nennenswerte Anhebung instationärer Störungen in der Grenzschicht beobachtet, während die Anregung aber eine deutliche stationäre Deformation einbrachte. Daher ist es sehr wahrscheinlich, dass die beobachteten angehobenen instationären Störungen durch mechanische Phänomene am Motor oder Drehteller verursacht sind und somit eher eine Konsequenz der konkreten technischen Realisierung darstellen.

\section{Fazit}

Bezüglich der in der Zielsetzung (Abschnitt 1.2) formulierten Frage nach der Eignung der beiden untersuchten Aktuierungskonzepte für einen Einsatz im Rahmen der UFD-Methode kann geschlussfolgert werden, dass beide Aktuierungskonzepte in der Lage sind, die Kontrollmode mit signifikanter Amplitude anzuregen und diese Amplitude entlang eines eindeutigen Zusammenhangs mit der jeweiligen Anregungsamplitude zu steuern. Im Falle der Heizaktuierung konnte gezeigt werden, dass die Heizung nicht zu einer signifikanten Destabilisierung bzgl. instationärer Störungen geführt hat und die trotzdem beobachtete Anhebung instationärer Störungen einen Einsatz im Rahmen der UFD-Methode zur Transitionsverzögerung nicht beeinträchtigt. Somit wurde gezeigt, dass die Heizaktuierung in der Lage ist, die Kontrollmode in ausreichendem Maß isoliert von anderen Störungen einzubringen. Auf Grundlage der Erkenntnisse dieser Arbeit zur Heizaktuierung ist zu erwarten, dass eine Transitionsverzögerung mit der UFD-Methode möglich ist, sobald die Modelloberfläche keine langwelligen Rauigkeitsanteile enthält. Im Falle der Rotationsaktuierung kann die beobachtete zusätzliche Anregung instationärer Störungen einen Einsatz im Rahmen der UFD-Methode unmöglich machen, da diese Anregung in dem beobachteten Ausmaß den laminar-turbulenten Umschlag meistens stromauf verschieben wird. Da es sich bei dieser Anregung aber zusammenfassend nicht um eine inhärente Eigenschaft der Rotationsaktuierung handelt, ist zu erwarten, dass sie durch eine aufwendigere technische Realisierung deutlich reduziert und wahrscheinlich sogar vermieden werden kann. 


\subsection{Ausblick}

Das neu aufgebaute Prinzipexperiment der schiebenden ebenen Platte steht nun bereit für weitere experimentelle Untersuchungen zum laminar-turbulenten Umschlag in dreidimensionalen Grenzschichten. Neben Aktuierungskonzepten zur Transitionsverzögerung ist bspw. auch der Einfluss von zweidimensionalen Stufen und Spalten im querströmungsdominierten Transitionsprozess ein aktuelles Forschungsthema, in dem die neu aufgebaute Konfiguration bereits verwendet wird (siehe z.B. [64]).

Der Rezeptivitätsmechanismus zur Anregung stationärer QSI ist sowohl für die Heizanregung als auch die Rotationsanregung weitestgehend ungeklärt. Dabei ist zu erwarten, dass besonders durch die Kombination von numerischen Untersuchungen mit den Ergebnissen dieser Arbeit und ggf. zusätzlichen detaillierten Messungen im linearen Bereich der Störungsentwicklung stromab der Aktuierung Erkenntnisse zum jeweiligen Rezeptivitätsprozess gewonnen werden können. Ein weiterer Test zur vorgestellten Rezeptivitätshypothese für die Rotationsanregung wäre aber z.B. schon der Vergleich einer Anregung durch gleichsinnige Rotation der Drehteller mit spannweitigem Abstand $\lambda$ und einer Anregung durch abwechselnd gegensinnige Rotation mit spannweitigem Abstand $\lambda / 2$ (siehe Untersuchungen zum Drehsinn in Abschnitt 8.1.1).

Zur Transitionsverzögerung mithilfe der UFD-Methode wurden für beide Anregungskonzepte Möglichkeiten einer verbesserten technischen Realisierung identifiziert. Für beide Konzepte wären auch zukünftige Untersuchungen interessant, in denen die natürlich dominante stationäre QSI-Mode in ihrer Wellenlänge und Amplitude durch den Einsatz von DRE fixiert wird. Dabei sollte die mittlere Transitionslage im aktuierten Fall jeweils mit dem Fall inklusive dieser Fixierung als auch mit dem Fall ohne DRE verglichen werden. Die Erzeugung der MFD für die UFD-Methode erfordert zwar nicht direkt das bei Saric et al. [95] gewählte Verhältnis der Kontrollmodenwellenlänge zur Wellenlänge der natürlich dominanten Mode von zwei Dritteln. Die Untersuchungen im Fall der Heizanregung haben aber gezeigt, dass bei zu großer Differenz dieser Wellenlängen ungünstige nichtlineare Wechselwirkungen stattfinden können. Auch deshalb wäre es besonders interessant, ein Aktuierungskonzept mit anpassbarer Wellenlänge der Kontrollmode zu entwickeln. Ein solches Konzept wäre auch in der Lage, in einem gewissen Bereich variierender Anströmungsbedingungen zu funktionieren. 
Zusätzlich wurde in der Arbeit mit der unbeabsichtigten Reduktion der Kontrollmode aufgrund der Heizanregung eine andere Methode zur Transitionsverzögerung demonstriert, die auch näher untersucht werden könnte. Wird eine stationäre QSI-Mode mit derselben spannweitigen Wellenlänge wie die der natürlich dominanten Mode und mit einem destruktiven Phasenbezug $\mathrm{zu}$ dieser angeregt, ist mit einer Transitionsverzögerung zu rechnen. 


\section{Anhang}





\section{A.1 Positionen der Druckbohrungen}

\begin{tabular}{cccc} 
Druckbohrungsnr. & $x_{c} / c$ & Druckbohrungsnr. & $x_{c} / c$ \\
\hline 1 & 0.0012 & 22 & 0.2716 \\
2 & 0.0026 & 23 & 0.3108 \\
3 & 0.0040 & 24 & 0.3529 \\
4 & 0.0052 & 25 & 0.3921 \\
5 & 0.0068 & 26 & 0.4341 \\
6 & 0.0082 & 27 & 0.4761 \\
7 & 0.0098 & 28 & 0.5181 \\
8 & 0.0112 & 29 & 0.5602 \\
9 & 0.0128 & 30 & 0.6022 \\
10 & 0.0142 & 31 & 0.6414 \\
11 & 0.0182 & 32 & 0.6834 \\
12 & 0.0255 & 33 & 0.7255 \\
13 & 0.0342 & 34 & 0.7675 \\
14 & 0.0474 & 35 & 0.8092 \\
15 & 0.0643 & 36 & 0.8528 \\
16 & 0.0837 & 37 & 0.8950 \\
17 & 0.1091 & 38 & 0.9351 \\
18 & 0.1344 & 39 & 0.9655 \\
19 & 0.1652 & 40 & 0.9840 \\
20 & 0.1960 & & \\
21 & 0.2324 & &
\end{tabular}

Tabelle A.1.1: Profiltiefenpositionen der Druckbohrungen im Naseneinsatz und der Modellplatte. Druckbohrungen 1-21 sind im Naseneinsatz eingelassen, Druckbohrungen 22-40 in der Modellplatte. 


\section{A.2 Strömungsfeld bei $x_{c} / c=0.8 \mathrm{im}$ Referenzfall}

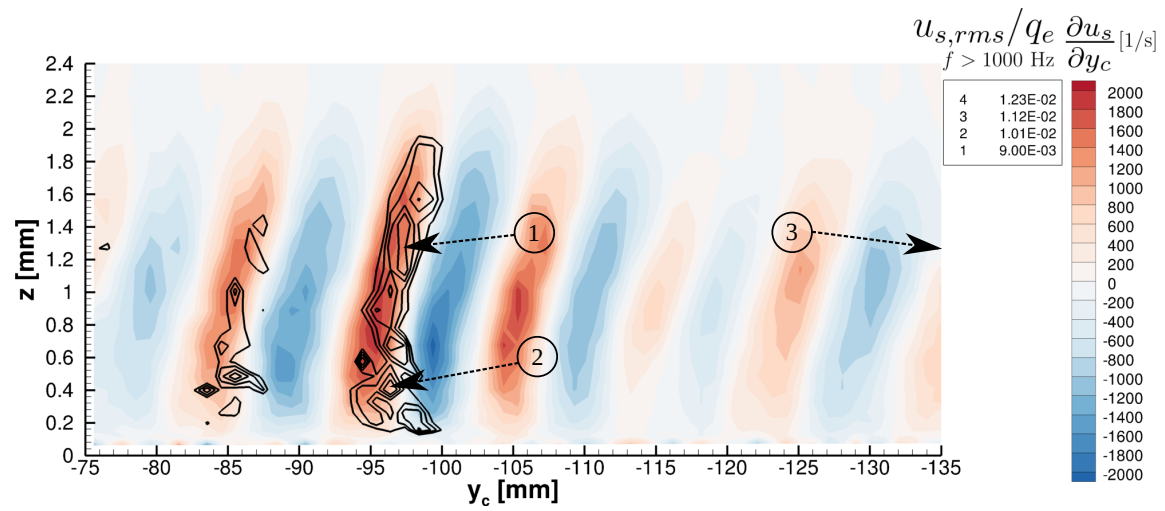

Abbildung A.2.1: Spannweitiger $u_{s}$-Gradient in Isokonturen und Effektivwerte von instationären $u_{s}$-Fluktuationen mit $f>1000 \mathrm{~Hz}$ bei $x_{c} / c=0.8$ mit Markierungen der Positionen, an denen in Abschnitt 6.3.4 Spektren instationärer Fluktuationen diskutiert werden 


\section{A.3 Rotations-Einzelanregung: Instationäre Störungen bei $f=f_{\text {rot }}$}

Um zu überprüfen, ob die Beobachtung der von $f_{\text {rot }}$ nahezu unabhängigen Anfangsamplitude $A_{0}$ für Störungen bei $f=f_{\text {rot }}$ auch auf andere einzeln betriebene Drehteller als Drehteller 9 zutrifft, wurde dieselbe Art der Auswertung wie für Abb. 8.1.8 auf gleiche Art und Weise für unterschiedliche Drehteller durchgeführt und das Ergebnis in Abbildung A.3.1 dargestellt. Zur Verdeutlichung der Unterschiede wurde dabei eine lineare Skala für die Ordinatenachse gewählt. Für jeden Drehteller ist $u_{s, L S T}^{\prime}(f)=A_{0} \exp \left(N_{\max }(f)\right)$ als gestrichelte Linie gleicher Farbe mit jeweils unterschiedlicher frequenzunabhängiger Anfangsamplitude $A_{0}$ eingezeichnet. Sie wurde jeweils so gewählt, dass $u_{s, L S T}^{\prime}(f)$ für $f_{r o t} \geq 500 \mathrm{~Hz}$ annähernd gut den beobachteten Amplituden bei der jeweiligen Frequenz entspricht.

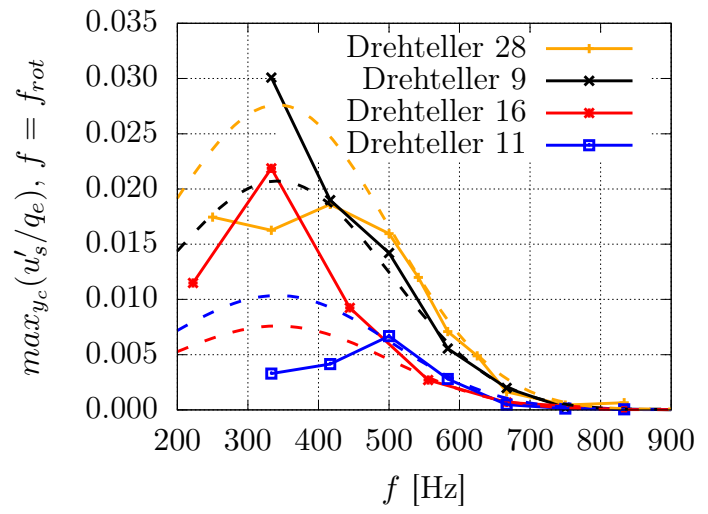

Abbildung A.3.1: Spannweitig maximal beobachtete Amplitude bei $f=f_{\text {rot }}$ für unterschiedliche Drehteller und unterschiedliche Werte von $f_{\text {rot }}$

Dabei zeigen sich für unterschiedliche Drehteller deutliche Unterschiede. Die Drehteller 9 und 16 zeigen für $f_{\text {rot }}>333 \mathrm{~Hz}$ einen qualitativ ähnlichen Verlauf, welcher allerdings bei Drehteller $16 \mathrm{zu}$ niedrigeren Frequenzen verschoben ist und daher bei Frequenzen $f<500 \mathrm{~Hz}$ nicht zu $u_{s, L S T}^{\prime}(f)$ passt. Stromab von den Drehtellern 11 und 28 werden Amplituden bei $f_{\text {rot }}$ beobachtet, die für $f_{\text {rot }}<500 \mathrm{~Hz}$ ebenfalls deutliche Unterschiede zu $u_{s, L S T}^{\prime}(f)$ aufweisen. Für $f_{\text {rot }} \geq 500 \mathrm{~Hz}$ passen die beobachteten Amplituden bei $f_{\text {rot }}$ für Drehteller 9, 11 und 28 näherungsweise $\mathrm{zu} u_{s, L S T}^{\prime}(f)$ bei frequenzunabhängiger Anfangsamplitude $A_{0}$. Diese Übereinstimmung weist darauf hin, dass die relevante 
instationäre initiale Störung zumindest in diesem Frequenzbereich mit nahezu frequenzunabhängiger Amplitude auftritt. Eine solche Übereinstimmung ist für Drehteller 16 nicht in gleicher Deutlichkeit zu beobachten. 


\section{A.4 Rotations-Einzelanregung: Überblick über instationäre Störungen}

(a) Drehteller 9

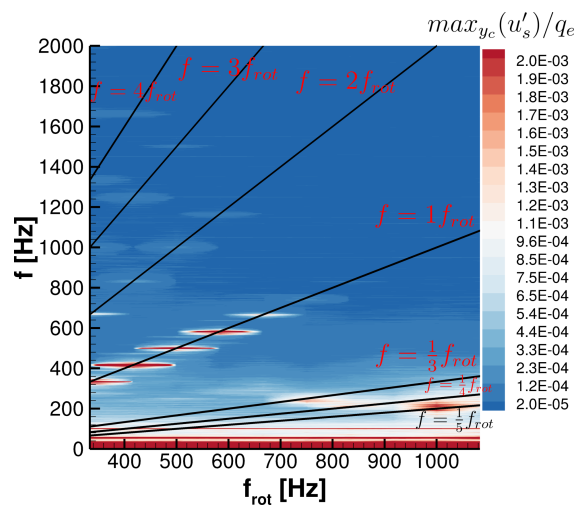

(c) Drehteller 16

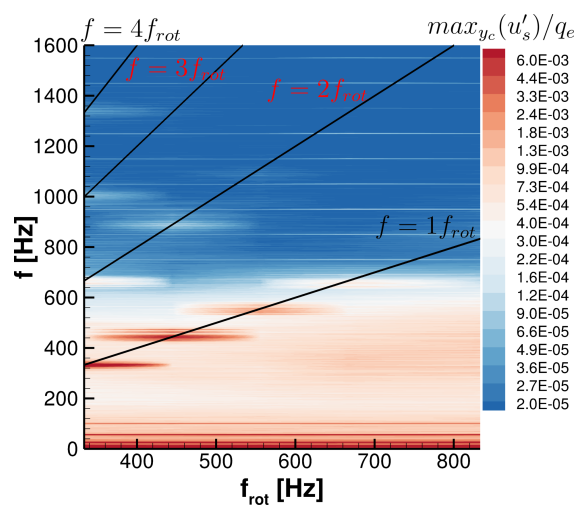

(b) Drehteller 11

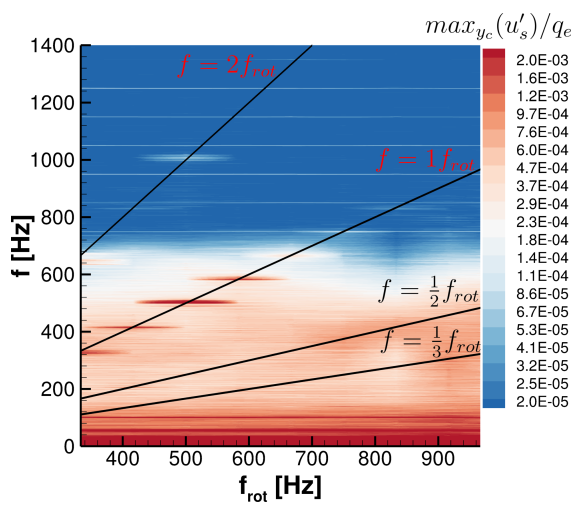

(d) Drehteller 28

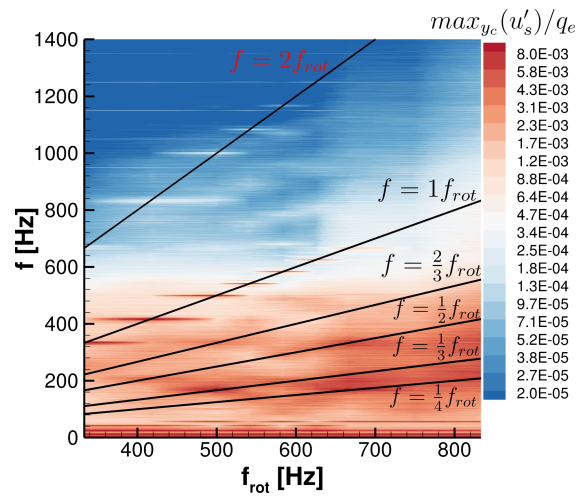

Abbildung A.4.1: Überblick über die Spektren der in Linienmessungen stromab von einzeln betriebenen Drehtellern beobachteten instationären $u_{s}$-Fluktuationen in Abhängigkeit der jeweiligen Rotationsfrequenz $f_{\text {rot }}$

Für einen qualitativen Überblick über die unterschiedlichen in der Grenzschicht stromab der Einzelanregung beobachteten instationären Phänomene sind die Spektren der spannweitig maximalen instationären $u_{s}$-Fluktuationsamplituden in Abbildung A.4.1 als Isokonturen abhängig von der jeweiligen 
Rotationsfrequenz $f_{\text {rot }}$ dargestellt. Mit eingezeichnete schwarze Linien markieren Positionen im Diagramm, an denen die Frequenz der in der Grenzschicht beobachteten Fluktuationen in einem festen Verhältnis zur Rotationsfrequenz steht. Dabei ist zu beachten, dass das Spektrum jeweils nur bei ausgewählten diskreten Rotationsfrequenzen bestimmt wurde. Bei der Darstellung als Isokonturen wird eine angehobene Schwankungsamplitude bei einer bestimmten Frequenz $f$ durch Interpolationsartefakte zwischen zwei Stützstellen als farbig hervorgehobene Linie parallel zur Abszisse dargestellt. Dies entspricht natürlich nicht den tatsächlich beobachteten Spektren, erhöht aber die Übersichtlichkeit im Vergleich zu einem einzeln farbig hervorgehobenen Punkt im Diagramm.

Auch hier fällt auf, dass die in diesem Abschnitt beschriebenen Phänomene instationärer Anregung durch Rotation stromab von unterschiedlichen einzeln betriebenen Drehtellern auch bei gleicher Rotationsfrequenz in stark unterschiedlichem Ausmaß beobachtet werden. Stromab von Drehteller 9 wird das Phänomen der über den Frequenzraum breit verteilten Amplitudenanhebung kaum beobachtet, während stromab von Drehteller 16 keine ausgezeichneten lokalen Maxima bei Subharmonischen der Rotationsfrequenz beobachtet werden. Die stärkste Anhebung instationärer Geschwindigkeitsfluktuationen inklusive aller bisher beschriebenen Phänomene wird stromab von Drehteller 28 beobachtet. 


\section{A.5 Drehteller ohne signifikante Anhebung instationärer Störungen}

(a)

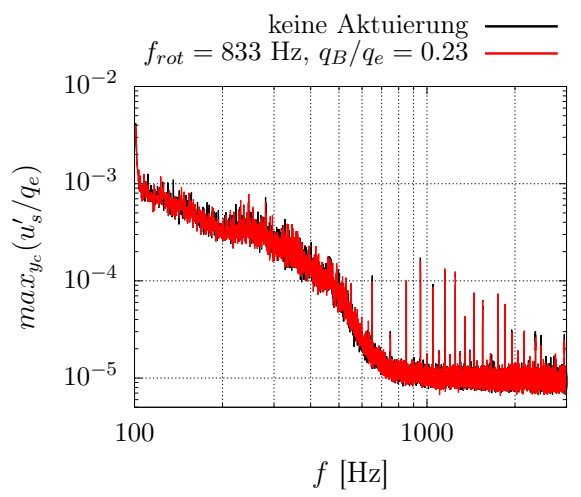

(b)

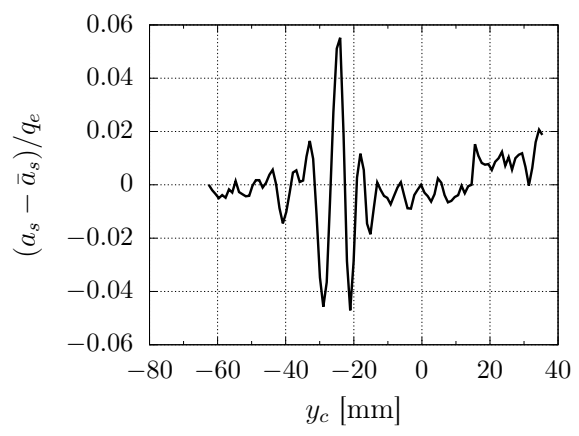

Abbildung A.5.1: (a) Spektrum spannweitig maximaler instationärer Fluktuationsamplituden stromab von Drehteller 22 , der mit $f_{\text {rot }}=833 \mathrm{~Hz}$ bzw. $q_{B} / q_{e}=0.23$ betrieben wurde, im Vergleich zum Fall ohne Aktuierung (beides gemessen bei $x_{c} / c=0.3$ ) (b) Die isolierte eingebrachte stationäre Grenzschichtdeformation in diesem Fall

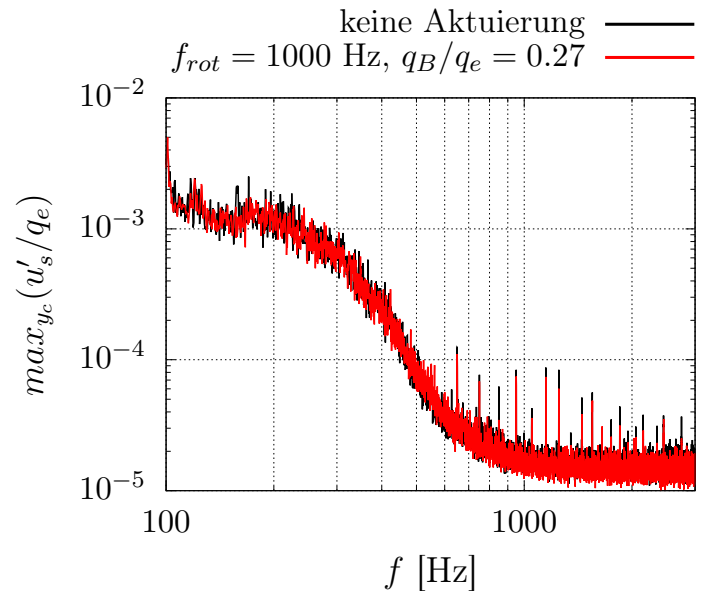

Abbildung A.5.2: Spannweitig maximale instationäre Fluktuationsamplituden stromab von Drehteller 20, auch bei $f_{\text {rot }}=1000 \mathrm{~Hz}$ nicht signifikant angehoben 


\section{Literaturverzeichnis}

[1] Abegg, C., Bippes, H., Boiko, A., Krishnan, V., Lerche, T., Pöthke, A., Wu, Y. und Dallmann, U.: Transitional Flow Physics and Flow Control for Swept Wings: Experiments on Boundary-Layer Receptivity, Instability Excitation and HLF-Technology. Aerodynamic Drag Reduction Technologies. Springer-Verlag Berlin Heidelberg, 2001, S. 199-206.

[2] Abegg, C., Bippes, H. und Janke, E.: Stabilization of Boundary-Layer Flows Subject to Crossflow Instability with the Aid of Suction. LaminarTurbulent Transition: IUTAM Symposium Sedona/AZ. Springer Berlin Heidelberg, 2000, S. 607-612.

[3] Alfeld, P.: A Trivariate Clough-Tocher Scheme for Tetrahedral Data. Computer Aided Geometric Design 1.2 (1984), S. 169-181.

[4] Arnal, D., Coustols, E. und Juillen, J. C.: Experimental and Theoretical Study of Transition Phenomena on an Infinite Swept Wing. La Recherche Aerospatiale (English Edition) 1984-4 (1984).

[5] Barth, H. P., Hein, S. und Rosemann, H.: Redesigned Swept FlatPlate Experiment for Crossflow-Induced Transition Studies. New Results in Numerical and Experimental Fluid Mechanics XI. Springer International Publishing, 2017, S. 155-165.

[6] Berger, C.: Einführung von künstlichen Störungen in die instabile, dreidimensionale, beschleunigte Grenzschichtströmung. Diplomarbeit. Universität Göttingen, 1990.

[7] Berthold, A.: Experimentelle Untersuchungen zur Entwicklung pneumatisch eingebrachter Störungen in einer dreidimensionalen Grenzschicht. Masterarbeit. Technische Universität Berlin, 2016. 
[8] Bippes, H.: Basic Experiments on Transition in Three-Dimensional Boundary Layers Dominated by Crossflow Instability. Progress in Aerospace Sciences 35.4 (1999), S. 363-412.

[9] Bippes, H. und Lerche, T.: Transition Prediction in Three-Dimensional Boundary-Layer Flows Unstable to Crossflow Instability. 28th Fluid Dynamics Conference. AIAA 97-1906. AIAA, 1997.

[10] Bippes, H., Müller, B. und Wagner, M.: Measurements and Stability Calculations of the Disturbance Growth in an Unstable Three-Dimensional Boundary Layer. Physics of Fluids A 3.10 (1991), S. 23712377.

[11] Bippes, H. und Nitschke-Kowsky, P.: Experimental Study of Instability Modes in a Three-Dimensional Boundary Layer. AIAA Journal 28.10 (1990), S. 1758-1763.

[12] Borodulin, V. I., Ivanov, A. V. und Kachanov, Y. S.: Swept-Wing Boundary-Layer Transition at Various External Perturbations: Scenarios, Criteria, and Problems of Prediction. Physics of Fluids 29.094101 (2017).

[13] Borodulin, V. I., Ivanov, A. V., Kachanov, Y. S., Crouch, J. D. und Ng, L. L.: Criteria of Swept-Wing Boundary-Layer Transition and Variable $N$-Factor Methods of Transition Prediction. XVII International Conference on Methods of Aerophysical Research. 2014.

[14] Borodulin, V. I., Ivanov, A. V., Kachanov, Y. S. und Roschektaev, A. P.: Receptivity Coefficients at Excitation of Cross-Flow Waves by Free-Stream Vortices in the Presence of Surface Roughness. Journal of Fluid Mechanics 716 (2013), S. 487-527.

[15] Brinkema, R.: Experimentelle und numerische Untersuchung der Strömungsbedingungen in einem Windkanal. Masterarbeit. RWTH Aachen, 2015 .

[16] Bruun, H. H.: Hot-Wire Anemometry: Principles and Signal Analysis. Oxford science publications. Oxford University Press, 1995.

[17] Carpenter, A., Saric, W. S. und Reed, H.: Laminar Flow Control on a Swept Wing with Distributed Roughness. 26th AIAA Applied Aerodynamics Conference. AIAA 2008-7335. 2008.

[18] COCO - a Program to Compute Velocity and Temperature Profiles for Local and Nonlocal Stability Analysis of Compressible, Conical Boundary Layers with Suction. Techn. Ber. ZARM, 1998. 
[19] Cox, M. G., Dainton, M. P., Forbes, A. B., Harris, P. M., Schwenke, H., Siebert, B. R. L. und Wöger, W.: Use of Monte Carlo Simulation for Uncertainty Evaluation in Metrology. Advanced Mathematical and Computational Tools in Metrology V. World Scientific, 2001.

[20] Crouch, J.: Receptivity of Three-Dimensional Boundary Layers. 31st Aerospace Sciences Meeting. AIAA 93-0074. 1993.

[21] Deyhle, H.: Einfluß der äußeren Strömungsbedingungen auf den Transitionsprozeß einer dreidimensionalen Grenzschicht. Dissertation. Universität Hannover, 1993.

[22] Deyhle, H. und Bippes, H.: Disturbance Growth in an Unstable ThreeDimensional Boundary Layer and Its Dependence on Environmental Conditions. Journal of Fluid Mechanics 316 (1996), S. 73-113.

[23] Dörr, P. C. und Kloker, M. J.: Numerical Investigation of PlasmaActuator Force-Term Estimations from Flow Experiments. Journal of Physics D: Applied Physics 48.395203 (2015).

[24] Dörr, P. C. und Kloker, M. J.: Stabilisation of a Three-Dimensional Boundary Layer by Base-Flow Manipulation Using Plasma Actuators. Journal of Physics D: Applied Physics 48.285205 (2015).

[25] Dörr, P. C. und Kloker, M. J.: Transition Control in a Three-Dimensional Boundary Layer by Direct Attenuation of Nonlinear Crossflow Vortices Using Plasma Actuators. International Journal of Heat and Fluid Flow 61 (2016), S. 449-465.

[26] Dörr, P. C. und Kloker, M. J.: Crossflow Transition Control by Upstream Flow Deformation Using Plasma Actuators. Journal of Applied Physics 121.063303 (2017).

[27] Drela, M.: Two-Dimensional Transonic Aerodynamic Design and Analysis Using the Euler Equations. Dissertation. Massachusetts Institute of Technology, 1985.

[28] Drela, M.: Newton Solution of Coupled Viscous/Inviscid Multielement Airfoil Flows. 21st Fluid Dynamics, Plasma Dynamics and Lasers Conference. AIAA 90-1470. AIAA, 1990.

[29] Drela, M.: Design and Optimization Method for Multi-Element Airfoils. Aerospace Design Conference. AIAA 93-0969. AIAA, 1993.

[30] Drela, M. und Giles, M. B.: Viscous-Inviscid Analysis of Transonic and Low Reynolds Number Airfoils. AIAA Journal 25.10 (1987), S. 13471355 . 
[31] Ehrensperger, K.: Verwendung der Applikation SAMS (Software für automatische Messdatenerfassung und Schrittmotorsteuerung). DLR. 2014.

[32] Farin, G.: Triangular Bernstein-Bézier Patches. Computer Aided Geometric Design 3.2 (1986), S. 83-127.

[33] Friederich, T. und Kloker, M.: Control of Crossflow-Vortex Induced Transition: DNS of Pinpoint Suction. 41st AIAA Fluid Dynamics Conference and Exhibit. AIAA 2011-3884. 2011.

[34] Gerhold, T., Evans, J. und Galle, M.: Technical Documentation of the DLR T-Code. Interner Bericht 223-97 A43. DLR, 1997.

[35] Gerhold, T., Galle, M., Friedrich, O. und Evans, J.: Calculation of Complex Three-Dimensional Configurations Employing the DLR-TauCode. 35th Aerospace Sciences Meeting and Exhibit. AIAA 97-0167. 1997.

[36] Gray, W.: The Effect of Wing Sweep on Laminar Flow. R.A.E. Technical Memorandum 255 (1952).

[37] Gregory, N., Stuart, J. T. und Walker, W. S.: On the Stability of Three-Dimensional Boundary Layers with Application to the Flow Due to a Rotating Disk. Philosophical Transactions of the Royal Society of London A 248.943 (1955), S. 155-199.

[38] Gregory, N. und Walker, W. S.: The Effect on Transition of Isolated Surface Excrescences in the Boundary Layer. Techn. Ber. 2779. Aeronautical Research Council: Reports und Memoranda, 1956.

[39] Groth, J. und Johansson, A. V.: Turbulence Reduction by Screens. Journal of Fluid Mechanics 197 (1988), S. 139-155.

[40] Hall, P.: The Linear Development of Görtler Vortices in Growing Boundary Layers. Journal of Fluid Mechanics 130 (1983), S. 41.

[41] Harris, C. R. u. a.: Array programming with NumPy. Nature 585.7825 (2020), S. 357-362.

[42] Hein, S.: Hypothese zur Anregung einer stationären Querströmungsinstabilität durch eine rotierende Scheibe. private Mitteilung. 2015.

[43] Hein, S.: Erweiterte Version der Software COCO. private Mitteilung. 2016.

[44] Hein, S.: Linear and Nonlinear Growth of Secondary Instabilities of Stationary Crossflow Vortices Studied by Parabolized Stability Equations. New Results in Numerical and Experimental Fluid Mechanics XI. Springer International Publishing, 2017, S. 219-228. 
[45] Hein, S., Bertolotti, F. P., Simen, M., Hanifi, A. und Henningson, D.: Linear Nonlocal Instability Analysis - the Linear NOLOT Code. Interner Bericht 223-94 A56. DLR, 1995.

[46] Hirschel, E. H. und Dallmann, U.: Vorschläge für die experimentelle Untersuchung des Übergangs laminar-turbulent in dreidimensionalen Grenzschichten. Interner Bericht 351-74/13. DFVLR, 1974.

[47] Hunter, J. D.: Matplotlib: A 2D graphics environment. Computing in Science \& Engineering 9.3 (2007), S. 90-95.

[48] JCGM: Evaluation of Measurement Data - Supplement 1 to the "Guide to the Expression of Uncertainty in Measurement"- Propagation of Distributions Using a Monte Carlo Method. Joint Committee for Guides in Metrology, 2008.

[49] Kachanov, Y. S. und Tararykin, O. I.: The Experimental Investigation of Stability and Receptivity of a Swept-Wing Flow. Laminar-Turbulent Transition: IUTAM Symposium Toulouse/France. Springer-Verlag Berlin Heidelberg, 1990, S. 499-509.

[50] Kachanov, Y.: Experimental Studies of Three-Dimensional Instability of Boundary Layers. 27th AIAA Fluid Dynamics Conference. AIAA 96-1978. AIAA, 1996.

[51] Kallinderis, Y., Khawaja, A. und McMorris, H.: Hybrid Prismatic/tetrahedral Grid Generation for Viscous Flows around Complex Geometries. AIAA Journal 34.2 (1996), S. 291-298.

[52] Kármán, T. V.: Über laminare und turbulente Reibung. ZAMM Journal of Applied Mathematics and Mechanics / Zeitschrift für Angewandte Mathematik und Mechanik 1.4 (1921), S. 233-252.

[53] Kim, J.-H., Forte, M. und Choi, K.-S.: Report on the Experimental Results on Transition Delay in the 'Juju' TRIN1 Wind Tunnel by the VR DBD Actuators Based on Wall-Normal Jets. Techn. Ber. ONERA, 2016.

[54] Koch, W., Bertolotti, F. P., Stolte, A. und Hein, S.: Nonlinear Equilibrium Solutions in a Three-Dimensional Boundary Layer and Their Secondary Instability. Journal of Fluid Mechanics 406 (2000), S. 131174 .

[55] Kohama, Y., Saric, W. S. und Hoos, J. A.: A High-Frequency, Secondary Instability of Cross-Flow Vortices That Leads to Transition. Proceedings of the Royal Aeronautics Society Conference on BoundaryLayer Transition and Control. 1991, S. 4.1-4.13. 
[56] Kolmogorov, A. N.: The Local Structure of Turbulence in Incompressible Viscous Fluid for Very Large Reynolds Numbers. Proceedings of the Royal Society A 434.1890 (1941), S. 9-13.

[57] Kraichnan, R. H.: Kolmogorov's Hypotheses and Eulerian Turbulence Theory. Physics of Fluids 7.11 (1964), S. 1723-1734.

[58] Kuryachii, A. P.: Control of Cross Flow in the Three-Dimensional Boundary Layer Using Space-Periodic Body Force Action. Fluid Dynamics 44.2 (2009), S. 233-239.

[59] Kurz, H. B. E. und Kloker, M. J.: Mechanisms of Flow Tripping by Discrete Roughness Elements in a Swept-Wing Boundary Layer. Journal of Fluid Mechanics 796 (2016), S. 158-194.

[60] Lemarechal, J.: Konturmessung an einem Naseneinsatz. private Mitteilung. 2018.

[61] Lerche, T.: Experimental Investigation of Nonlinear Wave Interactions and Secondary Instability in Three-Dimensional Boundary-Layer Flow. Advances in Turbulence VI. Springer Netherlands, 1996, S. 357-360.

[62] Lerche, T.: Experimentelle Untersuchung nicht-linearer Strukturbildung im Transitionsprozess einer instabilen dreidimensionalen Grenzschicht. Dissertation. Universität Hannover, 1997.

[63] Lerche, T.: Archivierte Messergebnisse zur Wanddruckverteilung im Prinzipexperiment der schiebenden ebenen Platte. 1998.

[64] Liegert, R.: Experimentelle Untersuchung des Einflusses von Oberflächenstufen auf das querströmungsdominierte Transitionsszenario. Masterarbeit. Ruhr-Universität Bochum, 2019.

[65] Liepmann, H. W., Brown, G. L. und Nosenchuck, D. M.: Control of Laminar-Instability Waves Using a New Technique. Journal of Fluid Mechanics 118 (1982), S. 187-200.

[66] Liepmann, H. W. und Nosenchuck, D. M.: Active Control of LaminarTurbulent Transition. Journal of Fluid Mechanics 118 (1982), S. 201204.

[67] Lippold, M.: 1-Meter Windkanal Betriebshandbuch. Ingenieurbüro Lippold. 2015.

[68] Lohse, J., Barth, H. P. und Nitsche, W.: Active Control of CrossflowInduced Transition by Means of In-Line Pneumatic Actuator Orifices. Experiments in Fluids 57.124 (2016). 
[69] Lohse, J., Berthold, A. und Nitsche, W.: Experimental Studies on the Receptivity of Stationary Crossflow Modes to Pneumatic Actuation. New Results in Numerical and Experimental Fluid Mechanics XI. Springer International Publishing, 2017, S. 263-273.

[70] Lohse, J.: Aktive Beeinflussung von Querströmungsinstabilitäten mit pneumatischen Aktuatoren. Dissertation. Technische Universität Berlin, 2017.

[71] Malik, M. R., Li, F. und Chang, C.-L.: Nonlinear Crossflow Disturbances and Secondary Instabilities in Swept-Wing Boundary Layers. IUTAM Symposium on Nonlinear Instability and Transition in ThreeDimensional Boundary Layers. Springer Netherlands, 1996, S. 257266.

[72] Malik, M. R., Li, F., Choudhari, M. M. und Chang, C.-L.: Secondary Instability of Crossflow Vortices and Swept-Wing Boundary-Layer Transition. Journal of Fluid Mechanics 399 (1999), S. 85-115.

[73] Melber-Wilkending, S., Schrauf, G. und Rakowitz, M.: Aerodynamic Analysis of Flows with Low Mach-and Reynolds-Number under Consideration and Forecast of Transition on the Example of a Glider. New Results in Numerical and Experimental Fluid Mechanics V. Springer-Verlag Berlin Heidelberg, 2006, S. 9-16.

[74] Morkovin, M. V.: On the Many Faces of Transition. Viscous Drag Reduction. Springer US, 1969, S. 1-31.

[75] Müller, B.: Experimental Study of the Travelling Waves in a ThreeDimensional Boundary Layer. Laminar-Turbulent Transition. SpringerVerlag Berlin Heidelberg, 1990, S. 489-498.

[76] Müller, B.: Experimentelle Untersuchung der Querströmungsinstabilität im linearen und nichtlinearen Bereich des Transitionsgebietes. Dissertation. Universität Karlsruhe (TH), 1990.

[77] Müller, B. und Bippes, H.: Experimental Study of Instability Modes in a Three-Dimensional Boundary Layer. AGARD Conference Proceedings: Fluid Dynamics of Three-Dimensional Turbulent Shear Flows and Transition. Bd. 438. 1989, S. 13.1-13.15.

[78] Nitsche, W. und Brunn, A.: Strömungsmesstechnik. Springer-Verlag Berlin Heidelberg, 2006.

[79] Nitschke-Kowsky, P.: Experimentelle Untersuchungen zu Stabilität und Umschlag dreidimensionaler Grenzschichten. Dissertation. Universität Göttingen, 1986. 
[80] Nosenchuck, D. M.: Passive and Active Control of Boundary Layer Transition. Dissertation. California Institute of Technology, 1982.

[81] Perry, A.: Hot-Wire Anemometry. Oxford science publications. Clarendon Press, 1982.

[82] Poll, D. I. A.: Transition in the Infinite Swept Attachment Line Boundary Layer. Aeronautical Quarterly 30.4 (1979), S. 607-629.

[83] Poll, D.: Some Observations of the Transition Process on the Windward Face of a Long Yawed Cylinder. Journal of Fluid Mechanics 150 (1985), S. 329-356.

[84] Radeztsky, R. H., Reibert, M. S. und Saric, W. S.: Effect of Isolated Micron-Sized Roughness on Transition in Swept-Wing Flows. AIAA Journal 37.11 (1999), S. 1370-1377.

[85] Rayleigh, L.: On the Stability, or Instability, of Certain Fluid Motions. Proceedings of the London Mathematical Society XI (1879), S. 57-72.

[86] Reed, H. L., Saric, W. S. und Arnal, D.: Linear Stability Theory Applied to Boundary Layers. Annual Review of Fluid Mechanics 28 (1996), S. 389-428.

[87] Reed, H. L. und Saric, W. S.: Stability of Three-Dimensional Boundary Layers. Annual Review of Fluid Mechanics 21 (1989), S. 235-284.

[88] Reibert, M.: Nonlinear Stability, Saturation, and Transition in Crossflow-Dominated Boundary Layers. Dissertation. Arizona State University, 1996.

[89] Reibert, M. S. und Saric, W. S.: Review of Swept-Wing Transition. 28th Fluid Dynamics Conference. AIAA 97-1816. AIAA, 1997.

[90] Reibert, M. S., Saric, W. S., Carrillo, R. B. J. und Chapman, K.: Experiments in Nonlinear Saturation of Stationary Crossflow Vortices in a Swept-Wing Boundary Layer. 34th Aerospace Sciences Meeting and Exhibit. AIAA 96-0184. 1996.

[91] Reynolds, O.: On the Dynamical Theory of Incompressible Viscous Fluids and the Determination of the Criterion. Philosophical Transactions of the Royal Society A: Mathematical, Physical and Engineering Sciences 186 (1895), S. 123-164.

[92] Robey, H. F.: The Nature of Oblique Instability Waves in Boundary Layer Transition. Dissertation. California Institute of Technology, 1986. 
[93] Rosemann, H.: Einfluß der Geometrie von Mehrfach-Hitzdrahtsonden auf die Meßergebnisse in turbulenten Strömungen. Dissertation. Technische Universität Clausthal, 1989.

[94] Saric, W. S., Carrillo, R. B. J. und Reibert, M. S.: Nonlinear Stability and Transition in 3-D Boundary Layers. Meccanica 33 (1998), S. 469487.

[95] Saric, W. S., Carrillo, R. B. J. und Reibert, M. S.: Leading-Edge Roughness As a Transition Control Mechanism. 36th AIAA Aerospace Sciences Meeting and Exhibit. AIAA 98-0781. 1998.

[96] Saric, W. S., Reed, H. L. und Kerschen, E. J.: Boundary-Layer Receptivity to Freestream Disturbances. Annual Review of Fluid Mechanics 34 (2002), S. 291-319.

[97] Saric, W. S., Reed, H. L. und White, E. B.: Stability and Transition of Three-Dimensional Boundary Layers. Annual Review of Fluid Mechanics 35 (2003), S. 413-440.

[98] Saric, W. S., West, D. E., Tufts, M. W. und Reed, H. L.: Flight Test Experiments on Discrete Roughness Element Technology for Laminar Flow Control. 53rd AIAA Aerospace Sciences Meeting. AIAA 20150539. 2015.

[99] Schlichting, H.: Zur Entstehung der Turbulenz bei der Plattenströmung. Nachrichten von der Gesellschaft der Wissenschaften zu Göttingen, Mathematisch-Physikalische Klasse (1933), S. 182-208.

[100] Schlichting, H. und Gersten, K.: Grenzschicht-Theorie. Springer-Verlag Berlin Heidelberg, 2006.

[101] Schuele, C. Y., Corke, T. C. und Matlis, E.: Control of Stationary Cross-Flow Modes in a Mach 3.5 Boundary Layer Using Patterned Passive and Active Roughness. Journal of Fluid Mechanics 718 (2013), S. $5-38$.

[102] Schülein, E.: Skin-Friction and Heat Flux Measurements in Shock/Boundary-Layer Interaction Flows. AIAA Journal 44.8 (2006), S. 1732 1741.

[103] Seiferth, R.: Vorausberechnung und Beseitigung der Schwingungen von Freistrahl-Windkanälen. Monographien über Fortschritte der deutschen Luftfahrtforschung seit 1939. Aerodynamische Versuchsanstalt Göttingen, 1946. 
[104] Serpieri, J., Venkata, S. Y. und Kotsonis, M.: Conditioning of CrossFlow Instability Modes Using Dielectric Barrier Discharge Plasma Actuators. Journal of Fluid Mechanics 833 (2017), S. 164-205.

[105] Serpieri, J., Venkata, S. Y. und Kotsonis, M.: Towards Laminar Flow Control on Swept Wings with AC-DBD Plasma Actuators As Active Roughness. 55th AIAA Aerospace Sciences Meeting. AIAA 2017-1459. 2017.

[106] Shahriari, N., Kollert, M. R. und Hanifi, A.: Control of a Swept-Wing Boundary Layer Using Ring-Type Plasma Actuators. Journal of Fluid Mechanics 844 (2018), S. 36-60.

[107] Spalart, P. und Allmaras, S.: A One-Equation Turbulence Model for Aerodynamic Flows. 30th Aerospace Sciences Meeting and Exhibit. AlAA 92-0439. 1992.

[108] Tollmien, W.: Über die Entstehung der Turbulenz. Nachrichten von der Gesellschaft der Wissenschaften zu Göttingen, MathematischPhysikalische Klasse (1929), S. 21-44.

[109] Touré, P. S. und Schuelein, E.: Study of 2-D Shock-Wave / Turbulent Boundary Layer Interaction. 47th AIAA Fluid Dynamics Conference. AIAA 2017-4123. 2017.

[110] Virtanen, P. u. a.: SciPy 1.0: Fundamental Algorithms for Scientific Computing in Python. Nature Methods 17.3 (2020), S. 261-272.

[111] Wassermann, P.: Direkte numerische Simulationen zum querströmungsinduzierten laminar-turbulenten Umschlagprozess in einer dreidimensionalen Grenzschichtströmung. Dissertation. Universität Stuttgart, 2002 .

[112] Wassermann, P. und Kloker, M.: Mechanisms and Passive Control of Crossflow-Vortex-Induced Transition in a Three-Dimensional Boundary Layer. Journal of Fluid Mechanics 456 (2002), S. 49-84.

[113] White, E. B. und Saric, W. S.: Secondary Instability of Crossflow Vortices. Journal of Fluid Mechanics 525 (2005), S. 275-308.

[114] Wilczek, M. und Narita, Y.: Wave-Number-Frequency Spectrum for Turbulence from a Random Sweeping Hypothesis with Mean Flow. Physical Review E 86.6 (2012).

[115] Wolf, C.: 1-Meter-Kanal Göttingen (1MG): Benutzerhandbuch. DLR. 2016 . 
[116] Yadala, S., Hehner, M. T., Serpieri, J., Benard, N., Dörr, P. C., Kloker, M. J. und Kotsonis, M.: Experimental Control of SweptWing Transition through Base-Flow Modification by Plasma Actuators. Journal of Fluid Mechanics 844 (2018). 


\section{Lebenslauf}

\section{Persönliche Daten}

Name : Hans Peter Barth

Geburtsort : : Göttingen

Geburtsdatum : $\quad$ 12. April 1985

Familienstand : ledig

Staatsangehörigkeit : deutsch

\section{$\underline{\text { Schulische Ausbildung }}$}

2004 Abitur an der Robert-Koch-Schule Clausthal-Zellerfeld

\section{Hochschulausbildung}

2005 Studium der Physik an der Georg-August-Universität Göttingen

2008-2009 Studium der Physik an der Università degli Studi di Padova 2012 Diplom, Georg-August-Universität Göttingen

Titel der Arbeit:

Einfluss von gelöstem Wasserstoff auf die Bildung und Multiplikation von Versetzungen bei der Verformung von Palladium

2013 Wissenschaftlicher Mitarbeiter am Deutschen Zentrum für Luft- und Raumfahrt im Institut für Aerodynamik und Strömungstechnik, Abteilung Hochgeschwindigkeitskonfigurationen

seit 2015 Promotion unter der Betreuung von Prof. Dr. Andreas Dillmann an der Georg-August-Universität Göttingen 